\title{
Modified Firearm Discharge Residue Analysis utilizing Advanced Analytical Techniques, Complexing Agents, and Quantum Chemical Calculations
}

\author{
William J. Feeney \\ West Virginia University, wjfeeney@mix.wvu.edu
}

Follow this and additional works at: https://researchrepository.wvu.edu/etd

Part of the Analytical Chemistry Commons, Physical Chemistry Commons, Probability Commons, Statistical Models Commons, and the Theory and Algorithms Commons

\section{Recommended Citation}

Feeney, William J., "Modified Firearm Discharge Residue Analysis utilizing Advanced Analytical Techniques, Complexing Agents, and Quantum Chemical Calculations" (2021). Graduate Theses, Dissertations, and Problem Reports. 10302.

https://researchrepository.wvu.edu/etd/10302

This Dissertation is protected by copyright and/or related rights. It has been brought to you by the The Research Repository @ WVU with permission from the rights-holder(s). You are free to use this Dissertation in any way that is permitted by the copyright and related rights legislation that applies to your use. For other uses you must obtain permission from the rights-holder(s) directly, unless additional rights are indicated by a Creative Commons license in the record and/ or on the work itself. This Dissertation has been accepted for inclusion in WVU Graduate Theses, Dissertations, and Problem Reports collection by an authorized administrator of The Research Repository @ WVU.

For more information, please contact researchrepository@mail.wvu.edu. 
Modified Firearm Discharge Residue Analysis utilizing Advanced Analytical Techniques, Complexing Agents, and Quantum Chemical Calculations

William J. Feeney

Follow this and additional works at: https://researchrepository.wvu.edu/etd

Part of the Analytical Chemistry Commons, Physical Chemistry Commons, Probability Commons, Statistical Models Commons, and the Theory and Algorithms Commons 


\title{
Modified Firearm Discharge Residue Analysis utilizing Advanced Analytical Techniques, Complexing Agents, and Quantum Chemical Calculations
}

\author{
William Jansen Feeney
}

Dissertation submitted to the

Eberly College of Arts and Sciences

at West Virginia University

in partial fulfillment of the requirements for the degree of

Doctor of Philosophy in Chemistry

Suzanne Bell, Ph.D., Co-Chair

Tatiana Trejos, Ph.D., Co-Chair

Glen Jackson, Ph.D.

Blake Mertz, Ph.D.

Stephen Valentine, Ph.D.

C. Eugene Bennett Department of Chemistry

Morgantown, West Virginia

2021

Key Words: gunshot residue, firearm discharge residue, host molecules, orbitrap, ESI, complexing, crown ether, molecular modelling, liquid chromatography

Copyright 2021 William Jansen Feeney 


\section{Abstract \\ Modified Firearm Discharge Residue Analysis utilizing Advanced Analytical Techniques, Complexing Agents, and Quantum Chemical Calculations}

William Jansen Feeney

The use of gunshot residue (GSR) or firearm discharge residue (FDR) evidence faces some challenges because of instrumental and analytical limitations and the difficulties in evaluating and communicating evidentiary value. For instance, the categorization of GSR based only on elemental analysis of single, spherical particles is becoming insufficient because newer ammunition formulations produce residues with varying particle morphology and composition. Also, one common criticism about GSR practitioners is that their reports focus on the presence or absence of GSR in an item without providing an assessment of the weight of the evidence. Such reports leave the end-used with unanswered questions, such as "Who fired the gun?" Thus, there is a critical need to expand analytical capabilities and enhance the impact of the forensic scientist's conclusions. To maximize the evidential value of GSR evidence, detection methods exploiting modern advancements in instrumentation must be explored and developed.

The research here addresses multiple concerns within the community by increasing the evidentiary value of GSR while addressing limitations about current understanding of the behavior and interactions of GSR traces in various scenarios. Presented here is a sequential investigation of 1) the existing practices for GSR analysis, 2) the development and validation of an alternative analytical technique for enhanced detection of inorganic and organic GSR, 3) the occurrence of IGSR and OGSR in various subpopulations and the use of probabilistic interpretation of evidence, and 4) the use of theoretical calculations to study the host-guest chemistry involved in the proposed analytical method.

This collection of work reviews the current literature review and illustrates a trend to investigate emerging methods to enhance IGSR analysis with a wider emphasis on OGSR compounds. Combining IGSR and OGSR components increases the confidence of detecting GSR on a collected sample.

In this study, we demonstrate use of LC-MS/MS and host-guest chemistry to detect IGSR and OGSR components in a single instrument. One advantage afforded by the proposed method is the dual detection of IGSR and OGSR on the same sample under 20 minutes, which is about an order of magnitude faster than existing techniques, like scanning electron microscopy-energy-dispersive X-ray Spectrometry (SEM-EDS) analysis. Also, the wide use of LC-MS/MS technology at crime laboratories enables future technology transfer and implementation. This strategy is employed in a population study of over 400 authentic specimens to differentiate shooter from non-shooters from samples taken from a subject's hands. The prevalence of organic and inorganic gunshot residue is evaluated within two main subpopulations, 1) non-shooters, including groups with low- and highrisk of containing GSR-like residues, and 2) individuals involved in a firing event (shooters, bystanders, and shooters performing post-shooting activities). The subpopulations were investigated using both simple exploratory analyses to monitor the occurrence of GSR and 
machine learning algorithms for classification and class-prediction. Additionally, the probabilistic outputs resulting from the machine learning algorithm (neural networks) were used to assess the weight of evidence using likelihood ratios. Accuracy ranging from 90-99\% was obtained, depending on the population of interest with larger LRs observed in shooter's sets, proving substantial progress to conventional categorical approaches

Finally, the host-guest chemical interactions from this study were further investigated using theoretical calculations. A quantum mechanical approach (DFT) was utilized to monitor the noncovalent, electrostatic interactions between the 18-crown-6-ethers and other metallic ions including the alkali, alkali earth, and pnictogen groups. Additionally, oxygen atoms were replaced with other heteroatoms in these macrocycles to study the thermochemical binding. It was found that electronegativity proved to be the greatest factor influencing the strength of binding followed by the size of the interacting cation.

Overall, the development of novel analytical methods for GSR detection, the application of ground-breaking statistical methods to interpret GSR evidence using artificial intelligence (neural networks) and likelihood ratios to estimate the weight of the evidence, and the understating of the host-guest chemistry of GSR species is anticipated to provide a needed leap of knowledge in the community. 


\section{Funding and Disclaimer}

This collection of works would not have been possible without the generous funding of the National Institute of Justice (NIJ). The funding opportunity associated with these works is Award \#2019-R2-CX-044, NIJ-STEM Graduate Research Fellowship, which was titled Validation of a Single Instrument, Single Protocol for the Detection of the Inorganic and Organic Constituents of Firearm Discharge Residues. This project was funded through January 2020-December 2021. The individuals associated with the fellowship were Tatiana Trejos (PI), Suzanne Bell (Chair) and William Feeney (PhD Student Fellowship Recipient). Importantly, the conclusions from each section were founded by me and the coauthors and disseminated to the scientific community in the form of review and research manuscripts. Hence, the opinions, findings, and conclusions are those of the authors and do not necessarily reflect those of the Department of Justice. 


\section{Dedication}

Dedicated to my loved ones who are with me every day and are with me in spirit.

Erin - I know being with me hasn't been easy (and I don't make it that way). Thank you for keeping me alive - in more ways than you can possibly imagine.

Mark - Words cannot express how much I miss you. Even now, I write this remorseful as I know that you will never read this. I can't thank you enough for making me think outside of the box and truly inspiring me. I'm sorry and miss you.

Gran and Papap- Everything ended too quickly and on terrible terms. Just wanted to say to both of you, thank you for being in my corner and helping me enjoy the simpler things in life. I miss you both every day.

Mom, Chris, and Joe - Thanks for holding down the fort and not getting too angry with me.

Dad - Thank you for reminding me how tranquil nature is.

Don't give up. We have all gone home in tears, we've all second-guessed why we do what we do. We've all felt like failures at times, and we have all wanted to quit at some point. Just remember, it's graduate school. You're smart and strong and you've made it this far. You can't expect it to be easy. 


\section{Acknowledgements}

Graduate school has been a trying and stressful journey. I certainly would not have made it on my own and with that, a special thanks and acknowledgements to the following groups and individuals is in order:

Family and Friends: Your journeys and successes gave me with inspiration and encouragement.

Brittany (Yeager) Stephens, Stephen Raso, and Kristin Kelly - You've made life in lab way more enjoyable in my first years. Watching you all leave was harder than you think. Thank you for being my pseudo family "Momma B", "Uncle Steve" and "Sister K" and opening your arms when I first entered this strange research world.

To my friends that are graduated and onto bigger and better things - There are so many of you to thank. Just know that you all helped me deal with my insecurities and helped me laugh.

To my newly adopted research family - You all made the transition into a new group easy. Thank you for being there and walking with me throughout me journey in the academic research realm. Know that you can call anytime, and I will assist in any way that I can.

To the C/Kourtneys - You both are great people. Unfortunately, the long nights in lab won't be the same without you there. Thank you for the FNAFs and laughs.

\section{Faculty:}

Becky Secrist - You are an amazing individual and have impacted so many graduate student's lives. The chemistry department was very lucky to have you. Thank you for everything you have helped me with over the years. I miss your smiling face and I hope you are enjoying the time with your family.

(Big) Bill Cunningham - You are... I guess an awesome individual to joke and vent to. The Forensics department is very lucky to have you. Thank you and I know you'll miss my face popping in your office at any time.

Last, but certainly not least, a special thanks goes to every one of my committee members - Dr. Suzanne Bell, Dr. Blake Mertz, Dr. Glen Jackson, Dr. Stephen Valentine, and Dr. Tatiana Trejos for reviewing my dissertation as well as serving on my graduate committee. Thank you all for your patience and wisdom throughout the years.

Dr. Bell, thank you for pushing and pushing me up and over the mountain of graduate school and reminding me every day that I forgot to check the "easy box".

Dr. Trejos, thank you for accepting this "head case" into your laboratory and teaching me how to become a better scientist. These past years have been the most inspiring times of my life and I am extremely fortunate to have learned from you.

Without you, this would not have been possible. 


\section{Table of Contents}

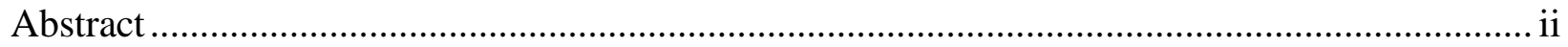

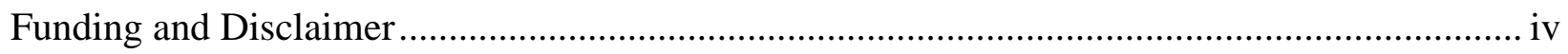

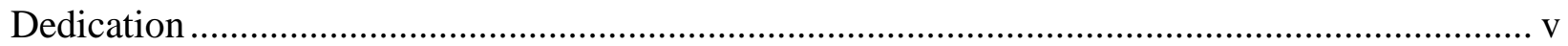

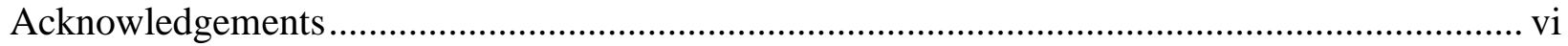

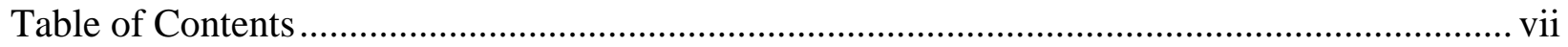

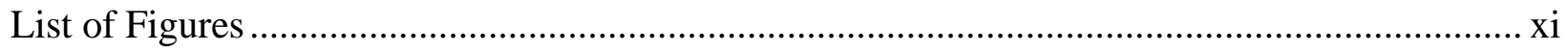

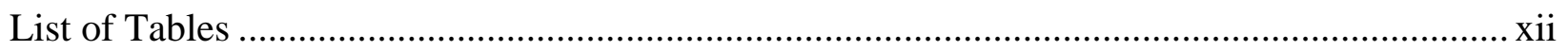

List of Abbreviations, Symbols, and Nomenclature ......................................................... xiii

CHAPTER 1: Problem Statement, Objectives, and Dissertation Structure .............................. 1

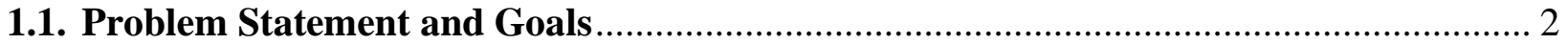

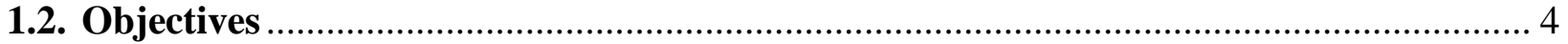

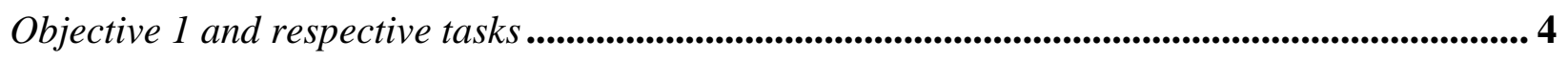

Objective 2 and respective tasks ..................................................................................................................... 4

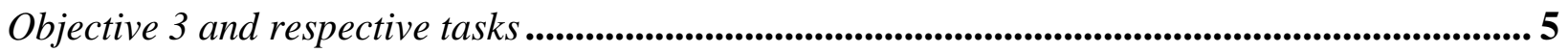

Objective 4 and respective tasks ............................................................................................................6 6

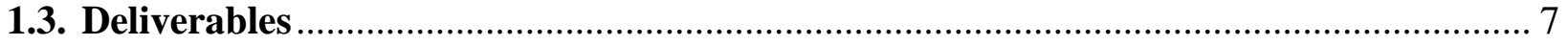

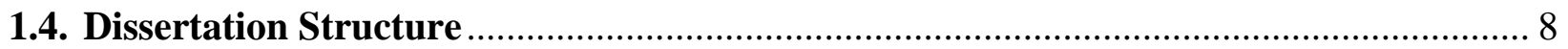

1.5. Implications for Criminal Justice and Practice in the United States ......................... 10

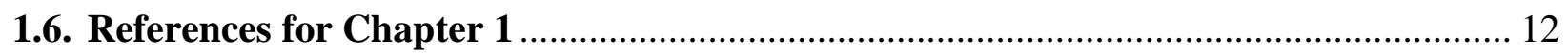

CHAPTER 2: Fundamentals of Gunshot Residues, Instrumentation, and Theoretical

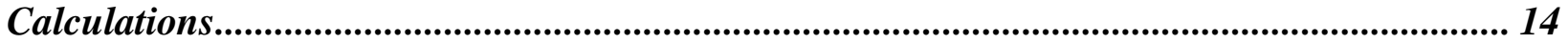

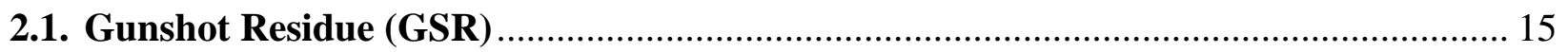

2.2. Liquid Chromatography-Tandem Mass Spectrometry (LC-MS/MS) ...................... 16

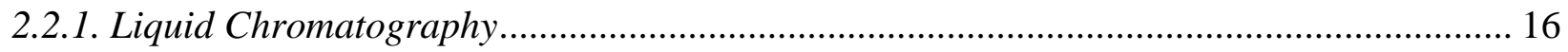

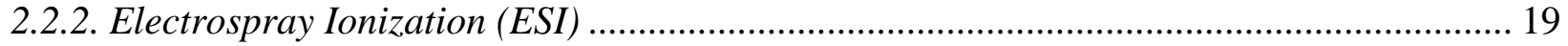

2.2.3. Triple Quadrupole Mass Analyzer (QQQ or $Q q Q)$........................................................ 24

2.2.4. High-Resolution mass spectrometry - Orbitrap ........................................................ 27

2.3. Overcoming challenges for metal analysis in LC-MS/MS ........................................ 28

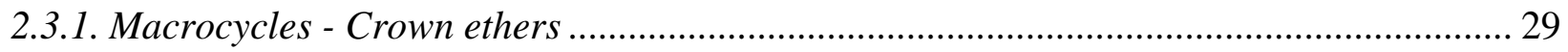

2.4. Strategies for investigating interactions of host-guest chemistry ............................. 30 


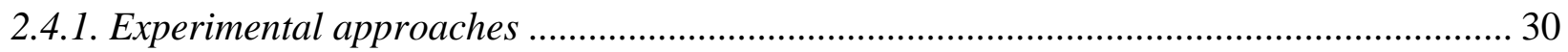

2.4.2. Theoretical calculations - Density Functional Theory (DFT) .......................................... 31

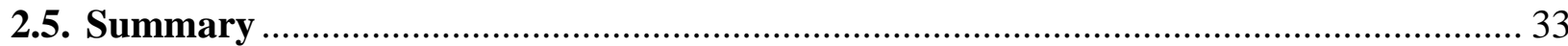

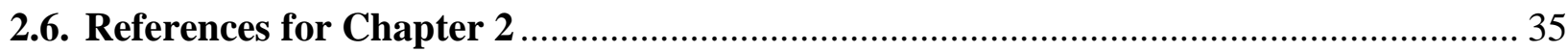

CHAPTER 3: Trends in Composition, Collection, Persistence, and Analysis of IGSR and

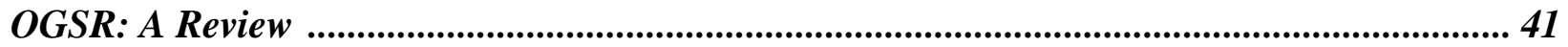

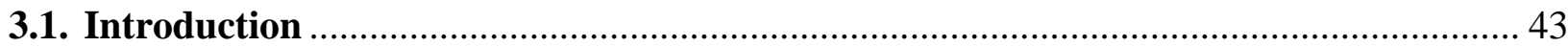

3.1. Inorganic and Organic Compositions of Modern Ammunitions.................................... 48

3.2. Common Gunshot Residue Sampling Media and Extractions ……………….............. 54

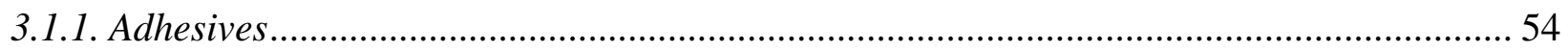

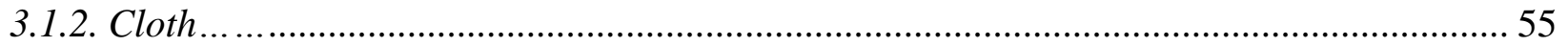

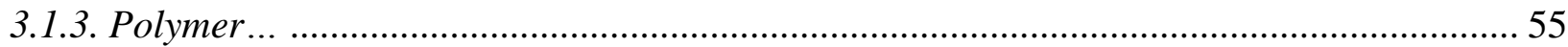

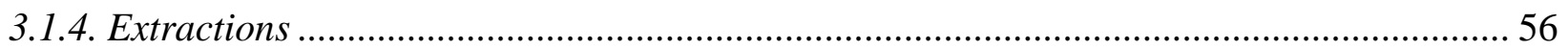

3.3. Deposition and Transfer Trends of Organic and Inorganic Gunshot Residues ............. 57

3.3.1. Transfer of Organic Gunshot Residue Compounds ....................................................... 59

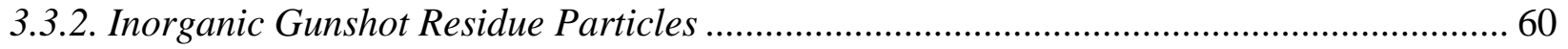

3.4. Persistence of Both Organic and Inorganic Components of Gunshot Residue ............. 63

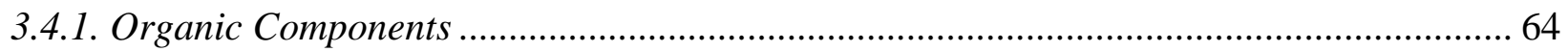

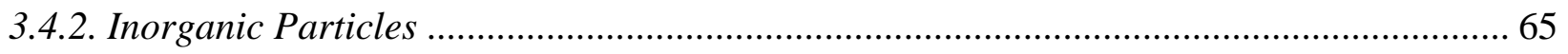

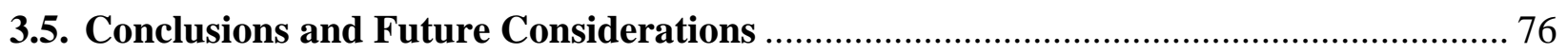

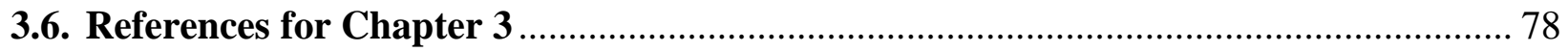

CHAPTER 4: Detection of Organic and Inorganic Gunshot Residues from Hands using Complexing Agents and LC-MS/MS ........................................................................................... 96

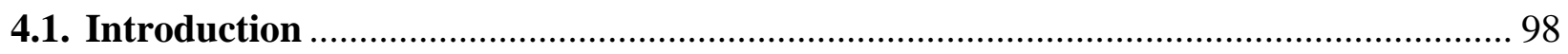

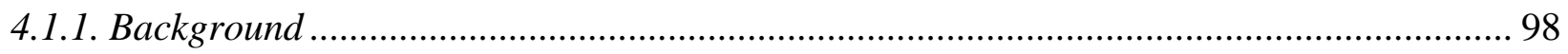

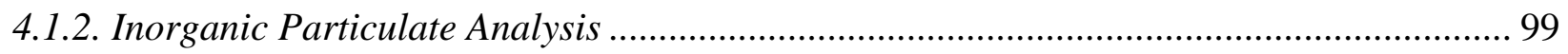

4.1.3. Organic Compound Analysis ....................................................................................... 100

4.1.4. Combined Analysis Methodologies .............................................................................. 101

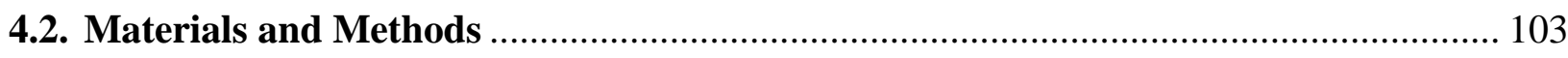

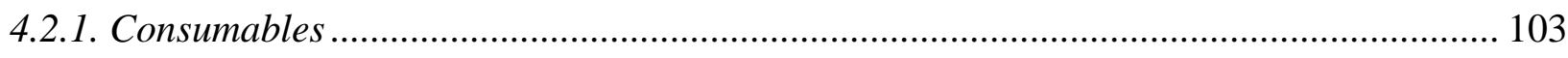

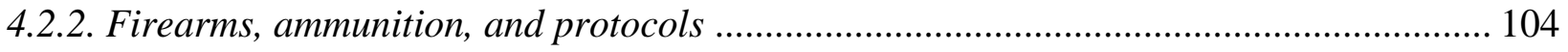

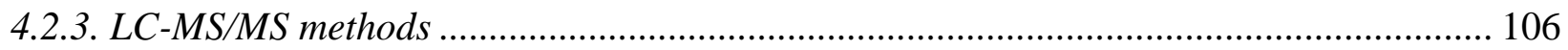

4.2.4. Comparisons with various analytical methods ............................................................... 109 
4.2.4.2. Electrochemical analysis

4.2.5. Extraction and collection of authentic samples for LC-MS/MS and multi-technique

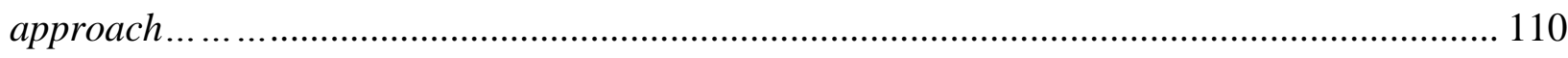

4.2.6. ICP-MS analysis for recovery study of collection substrates ...................................... 113

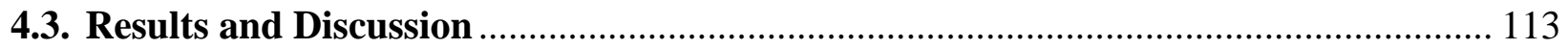

4.3.1. OGSR: Chromatography, CID experimentation, and observations ............................. 114

4.3.2. IGSR: Formation and identification of M-L complexes using HRMS .......................... 117

4.3.3. Analytical validation and figures of merit .................................................................. 123

4.3.4. Background and collection substrate considerations: recovery study and comparison to

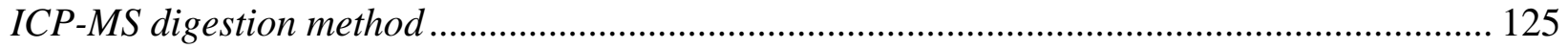

4.3.5. Cross-validation of recovery of IGSR via ICP-MS .................................................. 128

4.3.6. Workflow and cross-validation with screening methods .............................................. 129

4.3.7. Performance rates and criteria for authentic samples .............................................. 131

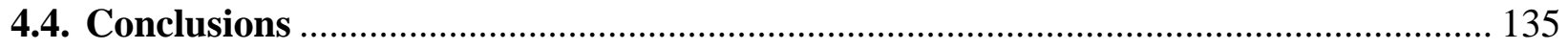

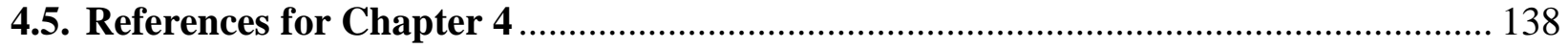

CHAPTER 5: Evaluation of Organic and Inorganic Gunshot Residues in Various Populations using LC-MS/MS ....................................................................................... 149

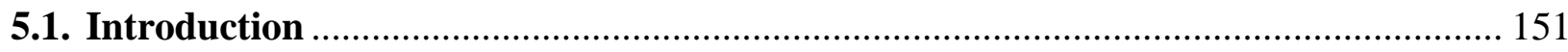

5.1.1. Current state and challenges of GSR detection ...................................................... 151

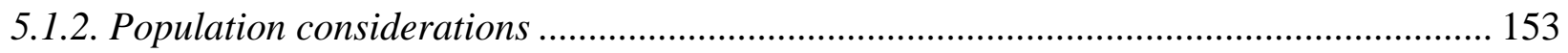

5.1.3. Neural Networks and likelihood ratios for GSR interpretation .................................... 154

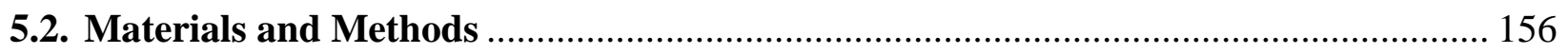

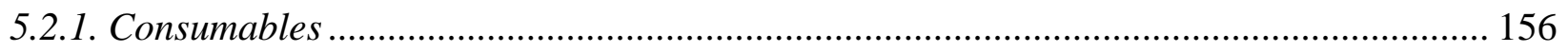

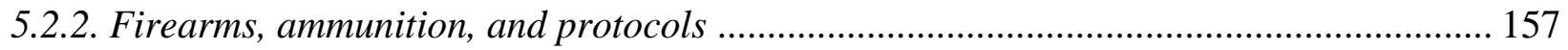

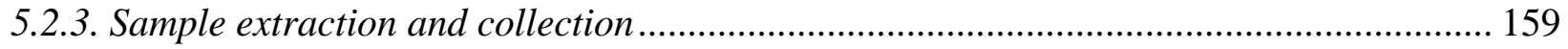

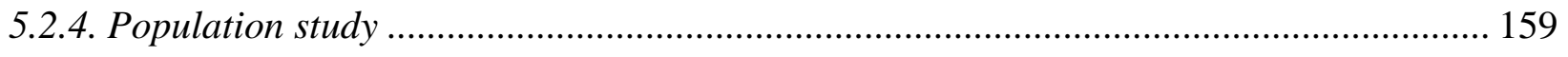

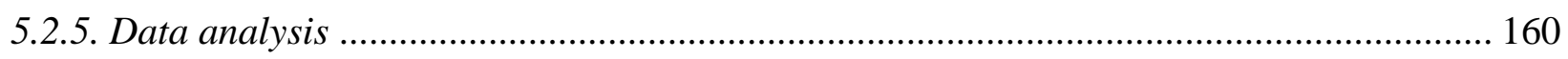

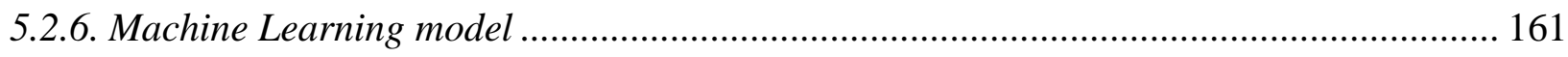

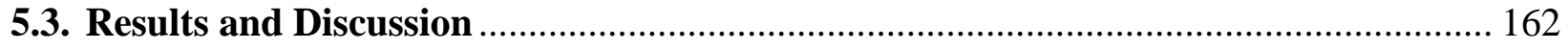

5.3.1. Occurrence of OGSR and IGSR on the sampled populations ..................................... 162

5.3.2. Neural network machine learning for GSR identification ............................................. 170

5.3.3. Likelihood ratios (LR) as probabilistic assessments of the weight of evidence ................ 172 


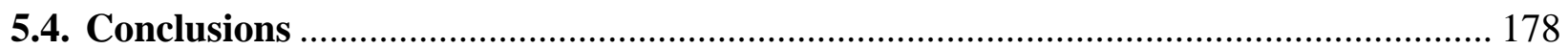

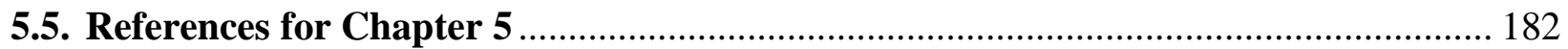

CHAPTER 6: Investigations into host-guest interactions with metal ions using DFT for

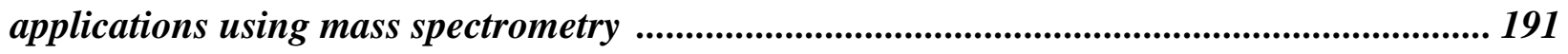

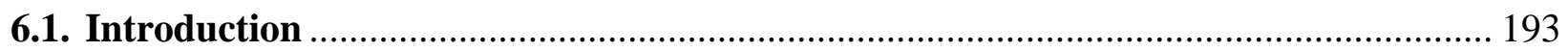

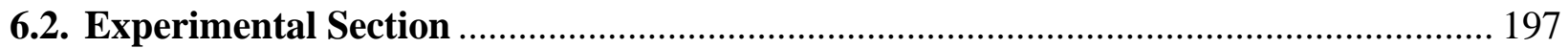

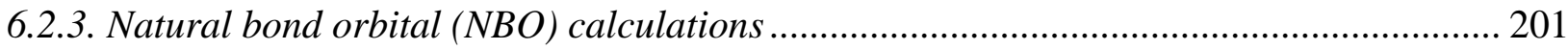

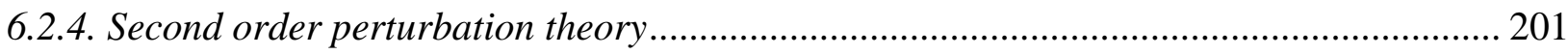

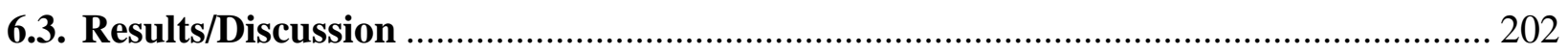

6.3.1. Confirmation of Structures via High-resolution Mass Spectrometry ............................... 202

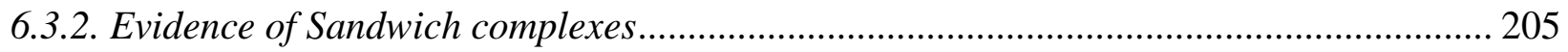

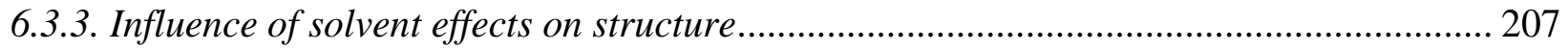

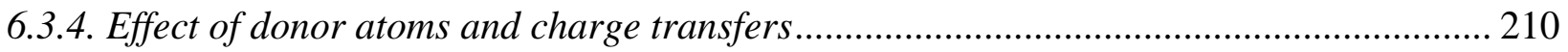

6.3.5. Metal guest influences on reactivity descriptors ............................................................ 214

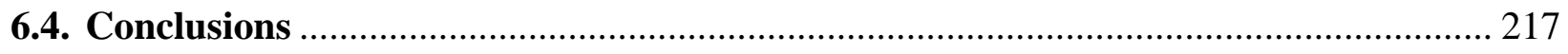

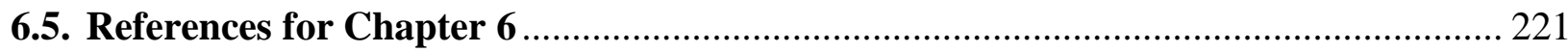

CHAPTER 7: Overall Conclusion and Future Directions ...................................................... 233

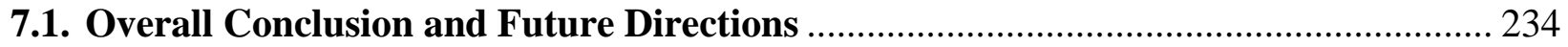

7.1.1. Objective 1: Review of the current literature associated with GSR ............................... 234

7.1.2. Objective 2: Validation and evaluation of a single instrument for sequential analysis of

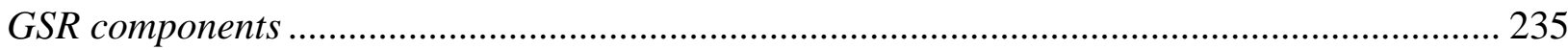

7.1.3. Objective 3: Investigation of the presence and prevalence of GSR in West Virginia within

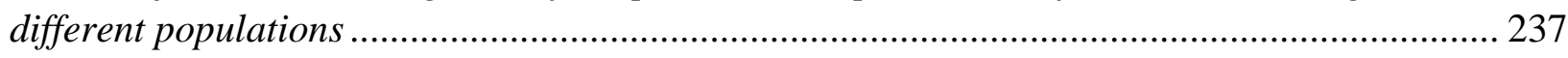

7.1.4. Objective 4: Investigations into host-guest interactions with metal ions using DFT for

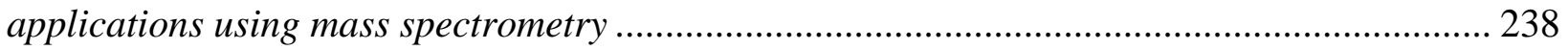

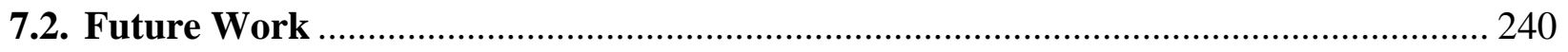

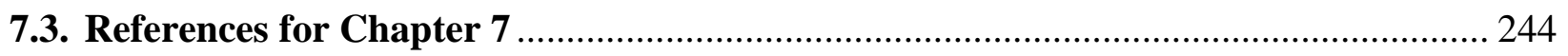

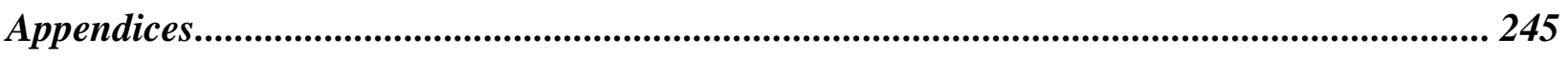

Appendix I-Chapter 4: Detection of Organic and Inorganic Gunshot Residues from Hands using Complexing Agents and LC-MS/MS - Supplemental Information .................................. 245

Appendix II - Chapter 5: Evaluation of Organic and Inorganic Gunshot Residues in Various Populations using LC-MS/MS - Supplemental Information .................................................... 248 Appendix III - Chapter 6: Investigations into host-guest interactions with metal ions using DFT for applications using mass spectrometry - Supplemental Information....................................2 250

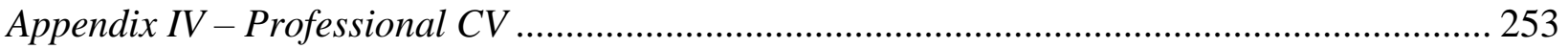




\section{List of Figures}

Figure 2.1: General schematic of electrospray ionization and Taylor cone formation.............. 21

Figure 2.2: Representation of two established electrospray ionization models - IEM and CRM..... 22

Figure 2.3: Recently proposed chain-ejection model by Konermann group................................. 23

Figure 2.4: Quadrupole mass filter representing ions following the set trajectory........................ 24

Figure 2.5: Schematic of QQQ with ESI source with a continuous multiplier tube......................... 25

Figure 2.6: Mathieu stability diagram for the stable regions for ions........................................... 26

Figure 2.7: Simple schematic of orbitrap mass analyzer with oscillating frequency....................... 27

Figure 2.8: Example of a "sandwich" structure with metal ion............................................... 31

Figure 3.1: Common sampling areas and techniques for gunshot residue evidence ...................... 56

Figure 4.1: Breakdown of extraction and analysis process and cross-validation techniques.......... 112

Figure 4.2: Chromatography separation of the seven OGSR compounds using PFP column........... 115

Figure 4.3: Orbitrap confirmation of $18 \mathrm{C} 6$ with $\mathrm{Ba}$ and $\mathrm{Pb}$ natural isotope distributions................. 117

Figure 4.4: Flow injection analysis of the various tartrate complexes with Sb in ESI- mode.......... 122

Figure 5.1: Boxplot comparisons between the low-risk, high-risk, and baseline shooter............ 163

Figure 5.2: Boxplot comparisons between low-risk, post-shooting activity, and baseline shooter... 165

Figure 5.3: Boxplot comparisons between the low-risk, bystander, and baseline shooter................ 167

Figure 5.4: OGSR and IGSR population comparison among all analytes tested............................. 169

Figure 5.5: Histogram and Tippet plot of shooter $(\mathrm{H} 1)$ and low-risk $(\mathrm{H} 2)$ subpopulation.................. 175

Figure 5.6: Histogram and Tippet plot of shooter $(\mathrm{H} 1)$, low-risk $(\mathrm{H} 2)$, and high-risk $(\mathrm{H} 2) \ldots \ldots \ldots . .176$

Figure 5.7: Histograms and Tippet plot of shooter (H1), low-risk (H2), and post-shooting (H1)..... 177

Figure 5.8: Histograms and Tippet plot of shooter (H1), low-risk (H2), and bystander $(\mathrm{H} 1) \ldots \ldots \ldots$.

Figure 5.9: Neural Network structure to train and validate authentic and low-risk populations.......... 179

Figure 6.1: Skeletal structure design of varying crown ethers simulated...................................... 199

Figure 6.2: Collected and simulated isotopic abundances from orbitrap of crown ether complexes... 204

Figure 6.3: Responses for barium and lead nitrate in the presence of crown ether via LC-MS/MS.... 206

Figure 6.4: Experimental example of a 1:2 M-L complex to a 1:1 M-L complex in ESI (+) mode... 209

Figure 6.5: Electrostatic distributions calculated for 18C6, aza-, thia-, and partial 18C6............ 211

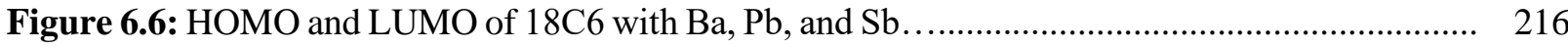

Figure 7.1: Typical locations for group substitution FRCs................................................... 241

Figure 7.2: Preliminary evaluation and interaction of carfentanil with cucurbit[7]uril ................... 242 


\section{List of Tables}

Table 3.1: Organic compounds that may contribute to GSR $\ldots \ldots \ldots \ldots \ldots \ldots \ldots \ldots \ldots \ldots . \ldots \ldots$

Table 3.2: Inorganic analytes that may contribute to GSR $\ldots \ldots \ldots \ldots \ldots \ldots \ldots \ldots \ldots \ldots \ldots \ldots \ldots$

Table 3.3a: OGSR focused methodologies.................................... 68

Table 3.3b: IGSR focused methodologies..................................... 70

Table 3.3c: Combined methodologies for GSR analysis......................... 75

Table 4.1: Orbitrap fragmentation patterns of metal-ligand complexes................ 118

Table 4.2: Figures of merit for OGSR compounds............................. 123

Table 4.3: Figures of merit for IGSR particulates.............................. 124

Table 4.4: OGSR recoveries from carbon tape and tesa ${ }^{\circledR}$ Tack..................... 127

Table 4.5: IGSR recoveries from carbon tape and tesa ${ }^{\circledR}$ Tack..................... 128

Table 4.6: Authentic shooting samples and success rates......................... 133

Table 4.7: Activity samples with combined rates............................. 134

Table 5.1: General breakdown of each population analyzed by LC-MS/MS analysis...... 160

Table 6.1: Metal cation characteristics that may affect binding..................... 206

Table 6.2: NBO analysis and charge transfers from $18 \mathrm{C} 6$ to $\mathrm{Pb}, \mathrm{Ba}$, and $\mathrm{Sb} . \ldots \ldots \ldots \ldots . . .213$

Table 6.3: Fully substituted macrocycles with oxygen, nitrogen, and sulfur atoms....... 215 


\section{List of Abbreviations, Symbols, and Nomenclature}

$\%$ RSD

$18 \mathrm{C} 6$

2,4-DNT

2-NDPA

4-NDPA

$\AA$

$\mathrm{ACN}$

ASTM

$\mathrm{Ba}$

$\mathrm{CE}$

CID

CRM

APCI

Da

DPA

EC

EDS or EDX

ESI

$\mathrm{eV}$

FDR

GC

GSR

$\mathrm{H}$

$\mathrm{H}_{2} \mathrm{O}$

H-G

eV

FDR

FTIR

GC

GSR

$\mathrm{H}$

H-G

HPLC

HRMS

ICP

IEM

IRB

$\mathrm{kV}$

LC

LOD

LOQ

$\mathrm{m} / \mathrm{z}$

$\mathrm{MC}$

$\mathrm{mg}$

$\min$
Percent Relative Standard Deviation

18-crown-6-ether

2,4-dinitrotoluene

2-nitrodiphenylamine

4-nitrodiphenylamine

Angstrom

Acetonitrile

American Society for Testing and Materials

Barium

Collision Energy

Collision Induced Dissociation

Charged-residue model

Atmospheric Pressure Chemical Ionization

Dalton

Diphenylamine

Ethyl Centralite

Energy Dispersive X-ray Spectrometry

Electrospray Ionization

Electron Volt

Firearm Discharge Residue

Gas Chromatography

Gunshot Residue

Enthalpy

Water

Host-Guest

Electron Volt

Firearm Discharge Residue

Fourier-transform infrared spectroscopy

Gas Chromatography

Gunshot Residue

Enthalpy

Host-Guest

High-Performance Liquid Chromatography

High-Resolution Mass Spectrometry

Inductively Couple Plasma

Ion Evaporation Model

Institutional Review Board

Kilovolt

Liquid Chromatography

Limit of Detection

Limit of Quantitation

Mass to Charge Ratio

Methyl Centralite

Milligram

Minute 


\begin{tabular}{|c|c|}
\hline $\mathrm{mL}$ & Milliliter \\
\hline IRB & Institutional Review Board \\
\hline LIBS & Laser Induced Breakdown Spectroscopy \\
\hline LR & Likelihood Ratio \\
\hline $\mathrm{MeOH}$ & Methanol \\
\hline MESP & Molecular Electrostatic Potential \\
\hline M-L & Metal-Ligand \\
\hline $\mathrm{mm}$ & Millimeter \\
\hline MRM & Multiple Reaction Monitoring \\
\hline MS & Mass Spectrometry \\
\hline $\mathrm{MS}^{\mathrm{n}}$ or MS/MS & Tandem Mass Spectrometry \\
\hline ng & Nanograms \\
\hline NG & Nitroglycerin \\
\hline NN & Neural Network \\
\hline N-NDPA & N-nitrosodiphenylamine \\
\hline $\mathrm{NBO}$ & Natural bond orbital \\
\hline OGSR & Organic Gunshot Residue \\
\hline $\mathrm{Pb}$ & Lead \\
\hline ppm & Parts Per Million \\
\hline $\mathrm{Q}_{1}$ & Quadrupole 1 \\
\hline $\mathrm{q}_{2}$ & Quadrupole 2 \\
\hline $\mathrm{Q}_{3}$ & Quadrupole 3 \\
\hline QC & Quality Control \\
\hline QqQ or QQQ & Triple Quadrupole \\
\hline Q-ToF & Quadrupole Time of Flight \\
\hline $\mathrm{RF}$ & Radio Frequency \\
\hline RMS & Root Mean Square \\
\hline RoME & Rates of Misleading Evidence \\
\hline Rt & Retention Time \\
\hline s & Second \\
\hline S.D. or sd & Standard Deviation \\
\hline $\mathrm{S} / \mathrm{N}$ or $\mathrm{SNR}$ & Signal-to-Noise Ratio \\
\hline $\mathrm{Sb}$ & Antimony \\
\hline SEM & Scanning Electron Microscopy \\
\hline SIMS & Secondary Ion Mass Spectrometry \\
\hline $\mathrm{T}$ & Temperature \\
\hline ToF & Time of Flight \\
\hline UHPLC & Ultra-High-Performance Liquid Chromatography \\
\hline$\mu g$ & Microgram \\
\hline$\mu \mathrm{L}$ & Microliter \\
\hline$\mu \mathrm{m}$ & Micrometer \\
\hline
\end{tabular}




\section{CHAPTER 1}

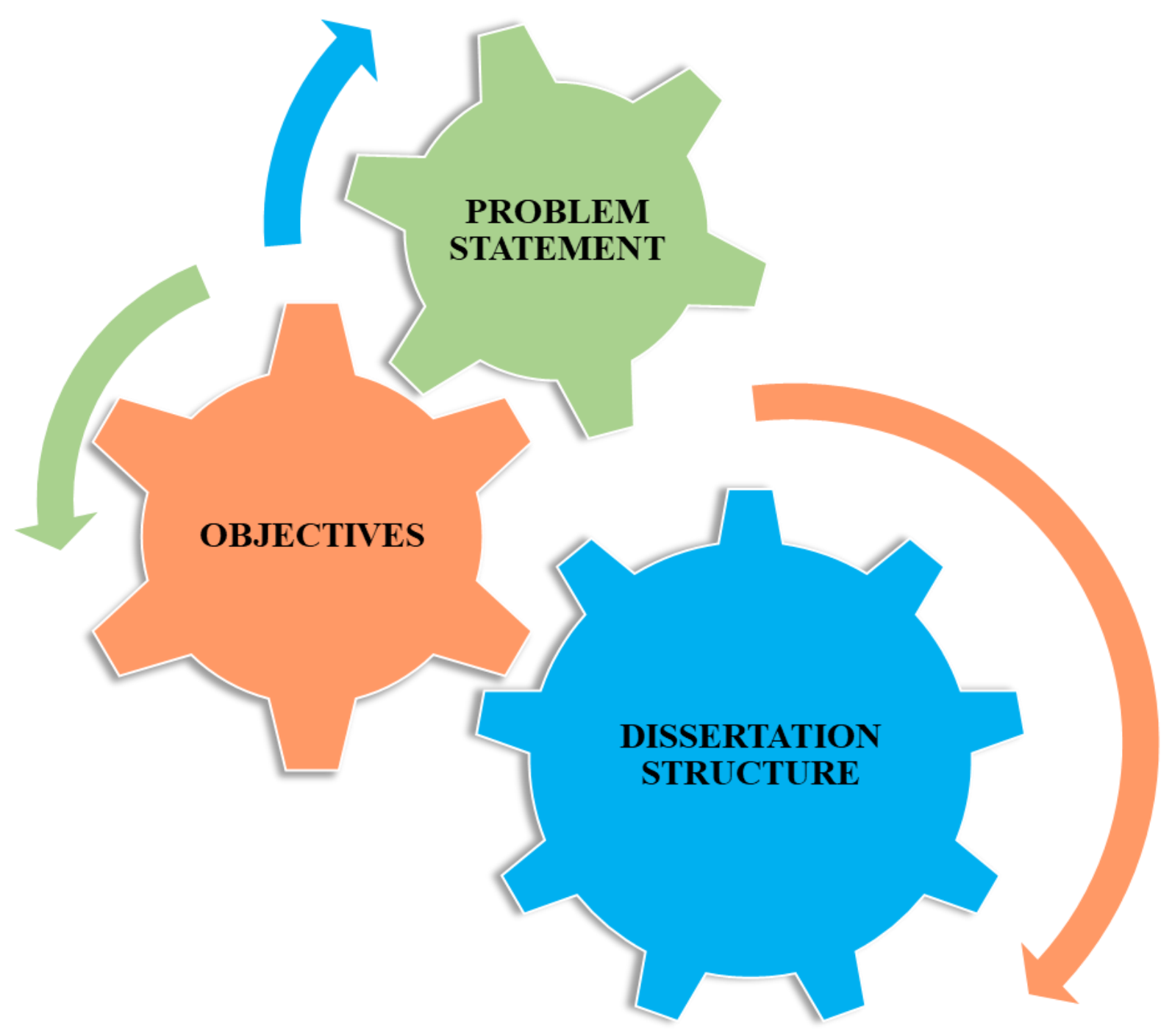

Problem Statement, Objectives, and Dissertation Structure 


\subsection{Problem Statement and Goals}

SEM-EDS is currently the gold standard for gunshot residue (GSR) analysis as per ASTM E1588-20 [1]. Nonetheless, with the prevalence of modern ammunition, the identification of particle morphology and elemental composition by SEM-EDS needs further orthogonal confirmatory tests. Collectively, the inorganic and organic components produced during the discharge of a firearm are known as GSR and combined provide stronger support of the differentiation of gunshot residues from other environmental and background residues. A comprehensive analysis of GSR often requires the use of two separate methodologies. For inorganic components (IGSR), SEM/EDS is the standard method used for morphology and elemental identification [1-3]. For organic molecules (OGSR), a consensus method has not been established, but recent research efforts have focused primarily on gas and liquid chromatography mass spectrometry for identification and quantitation $[2,3]$.

Other methods include the detection via ion mobility spectrometry (IMS), Raman spectroscopy, electrochemistry (EC), Laser-Induced Breakdown Spectroscopy (LIBS), Laser Ablation ICP-MS, and Time-of-Flight Mass Spectrometry [4]. The scientific community has hypothesized that evidentiary value increases by simultaneously detecting inorganic and organic components from a single sample. As a result, several approaches have been proposed to examine OGSR and IGSR using combinations of techniques, such as LIBS with electrochemistry, CMVGC/MS with LIBS, and LC-MS/MS with SEM/EDS [4]. The disadvantage of using multiple techniques is the significant time and resources required for preparation of additional samples, which are limited in casework. By using a single instrument, the shortcomings can be overcome through elimination of operations by multiple analysts and maintenance of several instruments.

This collection of work addresses the recent years' Technical Support Working Group (TSWG) list of research interests in trace evidence regarding the "evaluation of the 
detection and utility of organic gunshot primer residue," and the NIST-OSAC GSR subcommittee research needs on "comprehensive feasibility of organic gunshot residue analysis" and the "development of novel GSR methods for specific identification of shooters" [5,6].

The mechanisms of transfer and persistence of GSR are complex, and therefore the differentiation of residues deposited on shooters versus bystanders is not possible with current methodologies. The goal of this research is to validate an LC-MS/MS method for testing GSR evidence through the sequential analysis of organic and inorganic components using metal-ligand (M-L) complexes. This approach will provide a new tool for GSR analysts with the use of a common instrument found in most forensic laboratories.

The main hypothesis of this study is that the capabilities of the proposed method for simultaneous semi-quantitative detection of IGSR and OGSR components will help differentiate residues left on known shooters and bystanders and will enhance the overall reliability of firearmrelated evidence. A large population set with known "ground truth" regarding the individual's involvement with the discharge of a firearm (i.e., known shooter versus known non-shooter background population) was analyzed. The data was evaluated by categorical thresholds as well as machine learning algorithms and probabilistic frameworks.

Finally, simulation studies provided understanding of the complexing mechanisms between metal and ligand interactions. By utilizing quantum mechanical calculations, further insight can be gained about the sensitivity and binding affinities of various metal guests. Additionally, other factors including solvation polarizability (methanol, acetonitrile, water) and heteroatom substitution was investigated to monitor the effects of binding affinities and thermochemical values. 
Collectively, the fundamental and population studies, and the novel analytical and interpretation approaches proposed here are anticipated to increase capacity and narrow some of the knowledge gaps in the field of gunshot residues.

\subsection{Objectives}

The overall goal of this project was to develop a comprehensive strategy to enhance the reliability of the analysis and interpretation of gunshot residues (GSR). To accomplish this goal, four main objectives were outlined as described below.

Objective 1 and respective tasks

Objective 1. Perform an in-depth investigation on the major findings of current research approaches and practices within the most recent years regarding inorganic and organic gunshot residues collection, extraction, and detection.

Task 1.1. Generate a compilation of the main compounds and byproducts found on OGSR and IGSR of modern ammunition. Identify the use of each product within current traditional and non-traditional ammunition.

Task 1.2. Report and compare instrumentation utilized and their respective capabilities to detect OGSR, IGSR, or both. Additionally, report the findings, conditions, and parameters for ease of access and reference by practitioners and end-users.

Task 1.3. Identify deposition, transfer, and persistence challenges of IGSR particulates and OGSR compounds and evaluate where the current trends are shifting.

Objective 2 and respective tasks

Objective 2. Test the feasibility for dual characterization and validation of both OGSR and IGSR constituents using a single analytical instrument, liquid chromatography tandem mass spectrometry (LC-MS/MS). 
Task 2.1. Identify and confirm the major CID pathways of the studied OGSR compounds and self-assembled metal-ligand complexes using high- and low-resolution mass spectrometry instrumentation.

Task 2.2. Evaluate the figures of merit of the triple quadrupole mass spectrometry for all analytes under investigation using the Eurachem guideline [7]. Address the collection efficiencies of substrate used in previous proof-of-concept study (tesa Tack®) and the standard substrate (carbon adhesive mounted on an aluminum stub) using both LC-MS/MS and ICP-MS.

Task 2.3. Test the performance rates of the validated method with authentic shooting sample sets (with and without post-shooting activity). Test the feasibility of analyzing one sample with screening methodologies (LIBS and electrochemical methods) in conjunction with the newly developed confirmatory method.

\section{Objective 3 and respective tasks}

\section{Objective 3: Conduct a population study of GSR collected from known shooters and non-}

\section{shooters}

With this objective, we evaluate the feasibility to answer a major concern within the forensics community by observing the rate and prevalence of GSR within the general population. Furthermore, assess the performance rates and utilizing machine learning algorithms for generating likelihood ratios for more comprehensive objective evaluations by forensic practitioners.

Task 3.1. With recently validated instrument (LC-MS/MS), collect from various individuals from the general population of West Virginia. Collect from individuals who are either associated or dissociated with a firearm-related event. Additionally, collect from 
individuals whose professions may yield false-positive signals like GSR such as mechanics, farmer hands, and police officers.

Task 3.2. Collect and analyze samples from five subpopulations, including various background and shooters subsets: 1) 75 low-risk individuals who never interacted with or handled a firearm within the past 24 hours, 2) 75 non-shooter individuals that are considered high-risk due to their professions, 3) 75 samples collected from authentic shooters after recently discharging a firearm, 4) 75 samples from individuals performing activities after a discharge event, and 5) 75 samples collected from bystanders of a firing event.

Based on preliminary data, the sample size was selected for an estimated resulting power of this test of $95 \%$, providing a representative information of the prevalence of GSR within the WV population, and ultimately, assess the benefit of combining OGSR and IGSR data.

Task 3.3. Evaluate a data analysis model for the probabilistic assessment of the evidence. Curate the population dataset and further evaluate it through exploratory statistical analysis and machine learning algorithms. Neural networks are used to classify the samples and calculate the probability outputs of belonging to one of the five populations groups and monitored for their overall error rates. From those resulting probabilities, likelihood ratios are then computed to evaluate the presence of GSR at the source level.

\section{Objective 4 and respective tasks}

\section{Objective 4: Investigate binding affinities and sensitivities of guest-host species using density}

\section{functional theory}


Throughout this study, binding agents such as crown ethers have been utilized for IGSR detection for unique mass spectral signatures and transportation through the column and collision cells. However, their inability to associate with antimony $(\mathrm{Sb})$ is a glaring issue since it is a primary element utilized in traditional ammunition. Hence, a computational approach is used to highlight the underlying factors affecting binding of $\mathrm{Sb}$ to crown ether.

Task 4.1. Generate the chemical structures $\mathrm{x}, \mathrm{y}, \mathrm{z}$ coordinates (with and without metals) for both gas and solution phases (acetonitrile, water, methanol) to determine potential solvent effects. Additionally, heteroatoms such as $\mathrm{N}$ and $\mathrm{S}$ are incorporated in different positions of the ring structure to invoke and observe the effects of ring sizes and electronegativities.

Task 4.2. Employ density functional theory to calculate the non-covalent electrostatic interactions between the crown ether and heterocycles that interact with the metal ions.

Task 4.3. Evaluate the calculations to identify trends amongst different periodic groups using covariance and correlation exploratory analysis to understand the M-L behaviors.

\subsection{Deliverables}

This project was primarily funded by the National Institute of Justice (NIJ) under award \#2019-R2-CX-044 and this doctoral dissertation corresponds to one of the deliverables expected upon completion of this fellowship, which ends in December 2021. Objectives 1, 2 and 3 respond to the NIJ proposal. In addition to the financial, progress, and scholarly products required by the funding agency (i.e., publications and dissertation), other deliverables of this research include the dissemination of data collected within the forensic examiners and stakeholders. All data sets and methods for data processing created during this study will be made available to interested stakeholders and archived by the NIJ. As part of the dissemination strategy, we have published three scientific publications in peer-review journals, shared research results at six scientific 
meetings in the form of posters and oral presentations, and an additional publication about the computer simulations is in preparation.

\subsection{Dissertation Structure}

This dissertation describes the accomplishments of each of the major goals into four main chapters. Chapter 2 is a general introduction that highlights the main principles and practices utilized in the forensic community for GSR analysis as well as the primary techniques utilized throughout this study. Here, the reader is guided through the current understanding of gunshot residue, ionization sources and separation techniques, low- and high-resolution mass analyzers, theoretical calculations, and finally the species which sparked and assisted in this work. Also, this chapter describes the major findings and outcomes for the detection of organic and inorganic compounds of interest, as well as their shortcomings and future considerations for GSR evidence.

Chapter 3 describes the current findings and efforts displayed in the forensic research community for GSR detection, with a focus in composition, collection, persistence, and analysis of IGSR and OGSR. Here, the most recent articles pertaining to transfer, persistence, and combining inorganic and organic components for increased evidentiary value are summarized. Additionally, the articles' findings are reported by instrumentation and respective units of concentration or particle counts for a quick reference for both researchers and crime laboratory practitioners. This review fulfilled a gap of information for the current standings of GSR analysis as well as a brief overview for the direction and challenges experienced by the community. The content of this chapter was published in Forensic Chemistry as an open-access article in May 2020 (W. Feeney, C. Vander Pyl, S. Bell, T. Trejos, Trends in composition, collection, persistence, and analysis of IGSR and OGSR: A review, Forensic Chem. 19 (2020) 100250. https://doi.org/10.1016/j.forc.2020.100250) [4]. 
As a response to critical needs identified in the previous chapter, Chapter 4 describes the expansion and validation of a previous proof-of-concept study of our group for the dual detection of IGSR and OGSR using a single instrument, LC-MS/MS [8]. Here, the method was further optimized and validated, and the improved figures of merit are reported. Additionally, the interaction between the crown ethers and metal ions are further explained using a high-resolution mass analyzer. By exploring the feasibility of gathering data of inorganic and organic components from a single sample, one can reduce backlogs and expand knowledge on GSR analytes and enhance confidence in the results. Moreover, the validation of the method included the examination of authentic samples collected from shooters and non-shooters. For the authentic samples, a workflow was evaluated in conjunction with developing screening techniques for rapid and cost-effective approach that can permit triaging management of cases. The LC-MS/MS technique allowed sequential detection of OGSR then IGSR on the same sample. However, since OGSR are not routinely monitored in crime laboratories, the study also proposed a categorical thresholds-based criterion to identify GSR, and the respective performance rates are reported. Finally, several scenarios were evaluated to test the effectiveness of the newly developed method including individuals performing vigorous hand rubbing and cleansing, as well as running for a short duration after a firing event. The results of this work are published in Analytical Methods as an open-access journal in June 2021 [8]. This publication was selected as a Hot article and published in the journal's cover page ( W. Feeney, K. Menking-Hoggatt, C. Vander Pyl, C.E. Ott, S. Bell, L. Arroyo, T. Trejos, Detection of Organic and Inorganic Gunshot Residues from Hands using Complexing Agents and LC-MS/MS, Anal. Methods. (2021). https://doi.org/10.1039/d1ay00778e.) 
Chapter 5 expands to the validation study (Chapter 4) incorporating a larger population set of over 400 samples and reports the prevalence of OGSR and IGSR in various subpopulations, including bystanders and post-shooting sets, as well as background sets with low and high risk of containing GSR-like particles. Exploratory analysis was utilized to observe trends in the analytes within and between groups. A study of this size also allowed for the use of predictive machine learning methods for probabilistic interpretation of the data. Also, likelihood ratios were estimated and evaluated as a statistical approach to describe the weight of the evidence. This chapter was submitted for publication in Forensic Chemistry in October 2021.

Chapter 6 further explores the host-guest (H-G) interactions of the analytes of interest for this research by utilizing quantum mechanical calculations $[8,9]$. This study outlines the use of density functional theory (DFT) to monitor the noncovalent, electrostatic interactions in both gas and solution phases of various macrocycles. In this work, the thermochemical binding affinities were calculated using slew of metal cationic guests and were recorded to highlight potential trends and factors associated with complexation. This chapter will be submitted for publication in Analytical Chemistry.

Finally, Chapter 7 summarizes the overall conclusions of this dissertation and proposes future work and the application knowledge gained from the work to GSR and other forensic analytes - illicit drugs. This excerpt shows how screening methods can be benefitted from hostguest chemistry and how DFT can help visualize the interaction in play. Furthermore, theoretical, and experimental data can both be utilized to start building databases for additional GSR interpretation and future isomer identification of other analytes like drugs-of-abuse.

\subsection{Implications for Criminal Justice and Practice in the United States}

This research addressed specific research gaps identified by the Forensic Science TSWG and NIST-OSAC GSR subcommittee regarding the need for improvement of methods of detection 
and interpretation of gunshot residues. This study validated a novel method for simultaneously detecting IGSR and OGSR from the same sample and on a single instrument, expanding capabilities at crime laboratories. This study also provides the scientific community with a muchneeded body of knowledge regarding the occurrence of organic gunshot residues and the feasibility of incorporating OGSR into their workflow. The identification of orthogonal markers (inorganic and organic) is anticipated to reduce false positives and false-negative results. Moreover, probabilistic models will assist with quantitative assessment of the weight of the evidence, strengthening the analyst conclusions when writing reports and presenting evidence in court. The adoption of this methodology will modernize and streamline the current examination of GSR and enhance the reliability of evidence in court. 


\subsection{References for Chapter 1}

[1] S. Practice, S. Handbook, S. Practice, Standard Practice for Gunshot Residue Analysis by Scanning Electron Microscopy/Energy Dispersive X-Ray Spectrometry, ASTM Int. i (2020) 1-5. https://doi.org/10.1520/E1588-20.

[2] M. Manganelli, C. Weyermann, A.L. Gassner, Surveys of organic gunshot residue prevalence: Comparison between civilian and police populations, Forensic Sci. Int. 298 (2019) 48-57. https://doi.org/10.1016/j.forsciint.2019.02.050.

[3] C. Hofstetter, M. Maitre, A. Beavis, C.P. Roux, C. Weyermann, A.L. Gassner, A study of transfer and prevalence of organic gunshot residues, Forensic Sci. Int. 277 (2017) 241-251. https://doi.org/10.1016/j.forsciint.2017.06.013.

[4] W. Feeney, C. Vander Pyl, S. Bell, T. Trejos, Trends in composition, collection, persistence, and analysis of IGSR and OGSR: A review, Forensic Chem. 19 (2020) 100250. https://doi.org/10.1016/j.forc.2020.100250.

[5] O.G. Subcommittee, Comprehensive Feasibility of Organic Gunshot Residue Analysis, (2021). https://www.nist.gov/osac/osac-research-and-development-needs.

[6] O.G. Subcommittee, Development of New/Novel GSR Method(s) for Specific Identification of Shooters, (2021). https://www.nist.gov/osac/osac-research-and-developmentneeds.

[7] Eurachem, The Fitness for Purpose of Analytical Methods - A Laboratory Guide to Method Validation and Related Topics (2nd ed. 2014), Eurachem Guid. ISBN 978-91-87461-590. (2014) 1-70. https://doi.org/10.1016/S0014-2999(99)00500-2.

[8] W. Feeney, K. Menking-Hoggatt, C. Vander Pyl, C.E. Ott, S. Bell, L. Arroyo, T. Trejos, Detection of Organic and Inorganic Gunshot Residues from Hands using Complexing Agents and LC-MS/MS, Anal. Methods. (2021). https://doi.org/10.1039/d1ay00778e. 
[9] S. Bell, W. Feeney, Single shot, single sample, single instrument detection of IGSR and OGSR using LC/MS/MS, Forensic Sci. Int. 299 (2019) 215-222.

https://doi.org/10.1016/j.forsciint.2019.04.002. 


\section{CHAPTER 2}

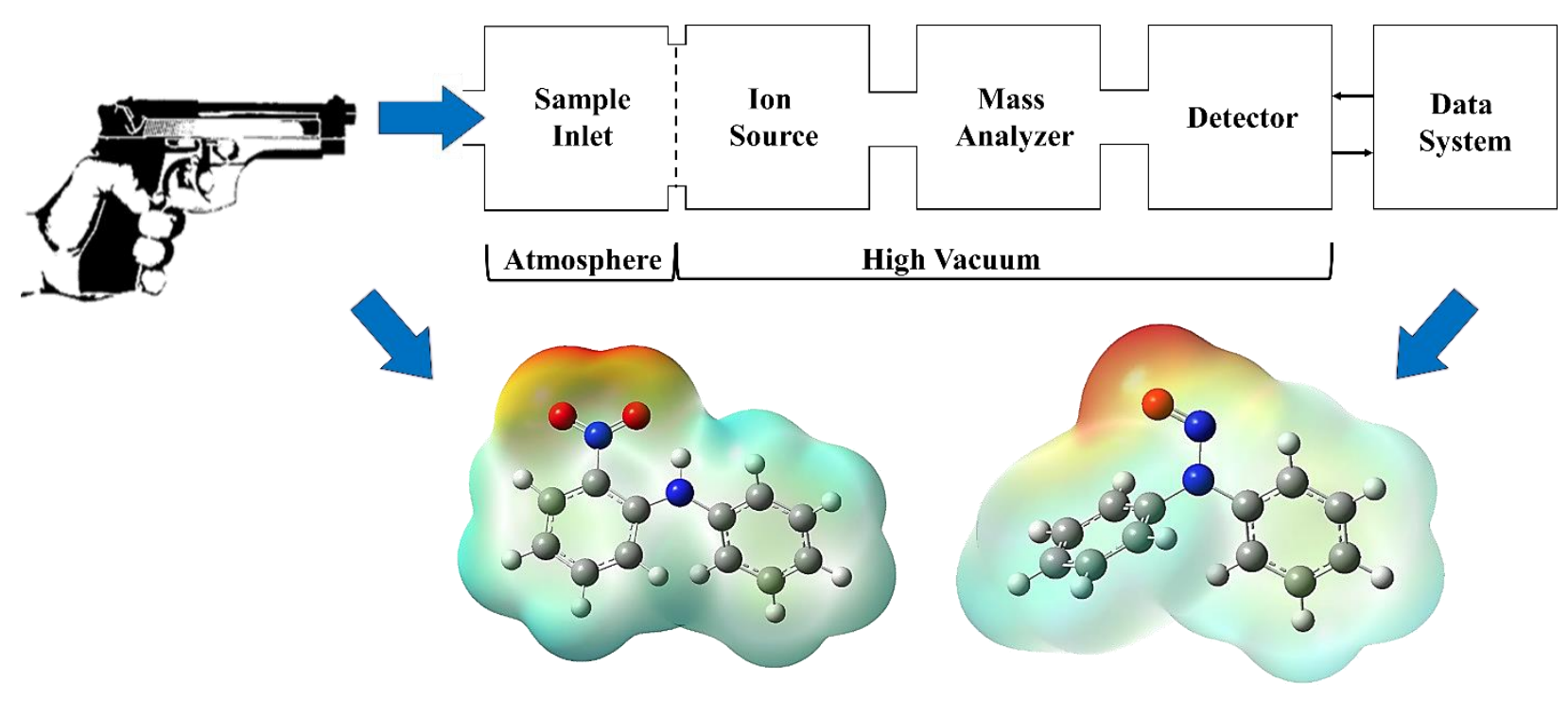

Fundamentals of Gunshot Residues, Instrumentation, and Theoretical Calculations 


\subsection{Gunshot Residue (GSR)}

Ever since the evolution from the flintlock pistol to Samuel Colt's multishot revolver, the interest of firearms has increased tremendously. However, it was not until the late 1970s when forensic scientists were truly interested in the clues obtained after a firing event. When a weapon is fired, residues are deposited on nearby surfaces, with the shooter's hand being of primary forensic interest. The deposited materials include vapor condensates, particles of unburned and partially burned propellant, and primer particulates. As such, it is a rich source of physical and chemical evidence that, to date, has not been exploited to its fullest probative value. For purposes of this work, GSR is divided into two categories: inorganic gunshot residue (IGSR) and organic compounds (OGSR).

These analytes arise from different components of the ammunition, OGSR compounds originate from the propellant and lubricant, whereas IGSR particulates emanate from the primer, bullet, and cartridge casing [1]. After a deflagration event, those analytes can be dispersed and spread onto surrounding surfaces, including hair, clothing, and hands. Due to the constituents' nature and various environmental factors, proficient collection and storage of the samples are essential to preserve the GSR compounds and increase the likelihood of detection. Typical indicators for IGSR are $\mathrm{Pb}, \mathrm{Ba}$, and $\mathrm{Sb}$, which are formed from the initial products lead styphnate $\left(\mathrm{C}_{6} \mathrm{HN}_{3} \mathrm{O}_{8} \mathrm{~Pb}\right)$, barium nitrate $\left(\mathrm{Ba}\left(\mathrm{NO}_{3}\right)_{2}\right)$, and antimony trisulfide $\left(\mathrm{Sb}_{2} \mathrm{~S}_{3}\right)$. Some of the more common OGSR analytes are diphenylamine (DPA), nitroglycerin (NG), ethyl centralite (EC), and 2,4-dinitrotoluene (2,4-DNT) [1]. Other compounds monitored, primarily formed by the combustion event and degradation of DPA, include 2-dinitrodiphenylamine (2-NDPA), 4nitrodiphenylamine (4-NDPA), and N-nitrosodiphenylamine (N-NDPA) [1]. These compounds' 
functional roles vary from detonation or blasting agents (explosives, oxidizers, fuel) to binding and performance materials (stabilizers and plasticizers).

Recent developments in instrumentation and reliable databases have allowed researchers and practitioners to create and methods to analyze said components. Currently, SEM-EDS is the preferred instrument for GSR detection and still resides as a standard consensus-based method ( $\mathrm{r}$ ASTM E1588-20 standard practice) [2]. This technique only analyzes IGSR particulate considering the morphology and chemical composition within single particles. However, novel methods and strategies have expanded the repertoire and capabilities of crime laboratories and how GSR behaves in different settings.

In this study, we performed exhaustive measurements and generated hypotheses using a common instrument found mainly in toxicology and drug crime laboratories, LC-ESI-MS/MS. In the following sections are some fundamentals of the operation of LC-MS/MS and how we overcame challenges with inorganic analysis and understanding those underlying mechanisms.

\subsection{Liquid Chromatography-Tandem Mass Spectrometry (LC-MS/MS)}

\subsubsection{Liquid Chromatography}

Liquid chromatography (LC) is a separation technique in which analytes interact with the stationary phase of the column and the mobile phase. Current liquid chromatography practices utilize packing particles (stationary phase) with various properties that invoke different responses. Because of these finely packed materials and column's dimensions itself, researchers can apply relatively high pressures, referred to as high-performance liquid chromatography (HPLC), while simultaneously achieving a high theoretical plate number for increased peak resolution [3]. Several factors influence the resolution given by a specified column, including 1) the mobile phase polarity, 2) the major chemistry interaction between analytes and the stationary phase, and 3) the 
column's dimensions and features. Hence, these factors can be further explained by the following formulae:

Equation 2.1

$$
K=\frac{c_{\text {stationary }}}{c_{\text {mobile }}}
$$

Where $K$ is the distribution factor and is dependent on the composition of the stationary phase $\left(c_{\text {stationary }}\right)$ and the characteristics of the mobile phase $\left(c_{\text {mobile }}\right)$. Additionally, Equation 2.1 can be further expanded to consider an analyte's retention given the parameters of the stationary and mobile phases. This consideration is represented in Equation 2.2:

Equation 2.2

$$
k_{A}^{\prime}=\frac{t_{R}-t_{0}}{t_{0}}
$$

Where $k^{\prime}$ is the retention factor of each analyte in a mixture and the terms $t_{R}$ and $t_{0}$ represent the retention times of a retained peak versus a sample eluting in the void volume, respectively. Since a column may possess unique qualities, one must consider how well analytes with similar properties or structures can be separated, which can be represented by Equation 2.3:

Equation 2.3

$$
\alpha=\frac{K_{B}}{K_{A}}=\frac{k_{B}^{\prime}}{k_{A}^{\prime}}=\frac{t_{R(B)}-t_{0}}{t_{R(A)}-t_{0}}
$$

Where $\alpha$ is the selectivity or separation factor and is dependent on the retention times of analytes $\left(t_{R(B)}\right.$ or $\left.t_{R(A)}\right)$. In other words, the selectivity is the ratio of two peak retention factors. Finally, researchers must consider how well a column's stationary phase is packed, i.e., the number of theoretical plates it possesses. These conceptual "plates" evaluate the effectiveness and performance of the column, specifically, and can be calculated by Equation 2.4: 
Equation 2.4

$$
N=16\left[\frac{t_{R}}{W}\right]^{2}
$$

The theoretical plates $(N)$ are tied to the column's length but are more impacted by the width of an analyte's peak $W$ in relation to its retention time $\left(t_{R}\right)$. With all these factors considered, the resolution can be calculated in Equation 2.5

Equation 2.5

$$
R_{s}=\left(\frac{k}{k+1}\right) *\left(\frac{\alpha-1}{\alpha}\right) *\left(\frac{\sqrt{N}}{4}\right)
$$

Where the retention factor $\frac{k}{k+1}$, the selectivity $\frac{\alpha-1}{\alpha}$, and the number of theoretical plates $\frac{\sqrt{N}}{4}$, influence peak shape and height as well as the quality of the data.

HPLC is often divided into two main operation modes, which corresponds to the polarity of the mobile and stationary phases - normal phase and reversed-phase. Instances where the stationary phase is more polar than the mobile phase is called normal phase liquid chromatography (NPLC) whereas the opposite is true for reversed-phase liquid chromatography (RPLC) [4,5]. Among these two strategies, RPLC is more popular due to the wide applicability and robust nature of the inexpensive $\mathrm{C} 18$ columns. However, if these columns do not assist with the separation of a particular species of interest, other columns have been adapted to help separation of species within a mixture. Some of these specialty column types include affinity, ion-exchange, size-exclusion, and hydrophilic interactions (HILIC) chromatography [3].

These various column strategies offer unique interactions and sometimes better separation versus the traditional nonpolar C18. For instance, affinity chromatography utilizes selective, noncovalent interactions between an analyte and specific molecules [6]. This type of chromatography is often used in biochemical applications to purify proteins bound to taggants. For ion-exchange 
chromatography, an ion exchange mechanism separates analytes centered around their respective charges and can be categorized into two main mechanisms - cation- and anion-exchange $[7,8]$. Conventionally, the stationary phase is an ion-exchange resin that carries charged functional groups that interact with oppositely charged groups of the compound to retain. In cation-exchange the stationary phase is negatively charged and interacts with a cation, whereas anion-exchange has a positively charged stationary phase and interacts with an anion.

Hydrophilic interaction chromatography (HILIC) utilizes a polar stationary phase in conjunction with an aqueous mobile phase. In HILIC, hydrophilic, polar, and charged compounds are retained preferentially compared with hydrophobic neutral compounds [9]. Finally, in sizeexclusion chromatography (SEC), molecules are separated according to their size [10]. For this strategy, the stationary phase is comprised of porous beads where smaller analytes molecules are trapped and removed from the flow. Larger molecules like biomolecules and proteins are not captured within the stationary phase and continue towards the detector. The smaller, trapped analytes then elute through the mobile phase.

Hence, countless types of column specific interactions are being tested and employed today depending on a laboratories' research needs. These unique adaptations have allowed liquid chromatography to become one of the most versatile separation techniques in analytical chemistry. Additionally, the flexibility of LC to couple to a wide variety detectors and mass analyzers has made it invaluable to many disciplines.

\subsubsection{Electrospray Ionization (ESI)}

An important component on mass spectrometry is the ionization source. Ever since the first conception to couple an ionization source to a mass analyzer in the late 1880 s, the ability to efficiently ionize analytes of interest has allowed many scientists to break barriers and overcome challenges never thought possible. Hard ionization methods employ energic species (atoms or 
electrons) to bombard and fragment various functional groups or exposed sites on analytes. One of the most popular sources is electron impact (EI) ionization which utilizes a beam of electrons formed by the filament and directed through the source at $70 \mathrm{eV}$ [11]. This mechanism removes electrons to form positive radical molecular ions and fragments which are often reproducible and are used to form extensive mass spectral libraries. This technique is widely popular in different fields and is often utilized in the GC/MS. However, this analytical strategy is limited due to the mass range $(<1000 \mathrm{Da})$ and the analyte's volatility.

From these earlier iterations of small molecule monitoring, scientists started to expand into other strategies to study complex analytes such as large biomolecules and proteins without major fragmentation. However, the primary concern was to preserve the natural state of these analytes to then investigate their primary functions and reactions in more complex systems. It was not until 1984 where Dole's and colleagues' concept of electrospray ionization (ESI) (Figure 2.1) was coupled to a mass analyzer (quadrupole) by Fenn and Yamashita to investigate the behavior of this soft ionization source [12].

In ESI, the analyte is dissolved in a solvent to be volatilized and transported to a capillary needle where a high positive or negative potential is applied. Because the ion formation involves extensive desolvation, volatile organic solvents such as methanol and acetonitrile, are often mixed with water. Furthermore, these solvents typically contain compounds like formic acid (FA) or acetic acid (AA) to not only increase conductivity but to also provide a source of protons to help facilitate the ionization process. 


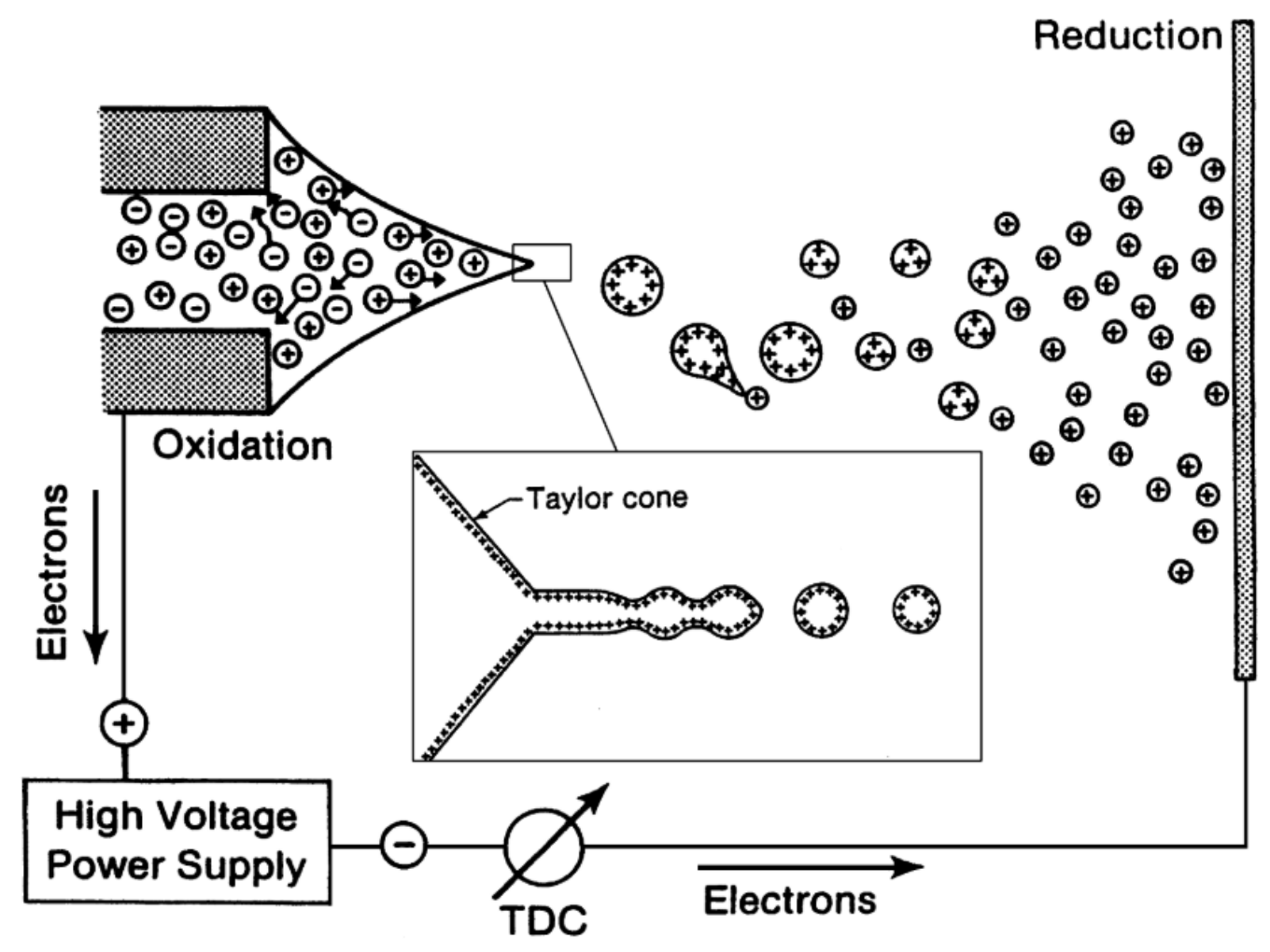

Figure 2.1: General schematic of electrospray ionization. As the charged solvent exits the end of the capillary, a high potential is applied and forms a Taylor cone. The charged droplets then are attracted to the orifice of the mass analyzer. Reproduced with permissions from [11]. J.H. Gross, Mass spectrometry, $2^{\text {nd }}$ ed., Springer-Verlag Berlin Heidelberg, 2011. https://doi.org/10.1007/9783-642-10711-5_4.

As the solvent traverses the capillary, the end of capillary is electrically charged with high electric potential in reference to a counter electrode (Figure 2.1). The end of the capillary is kept, typically, at 3-4 kV and the exposed liquid is exposed to an additional electric field [11]. That electric field causes charge separation and forms a cone termed Taylor cone. Once the solvent evaporates from a charged droplet, it becomes unstable upon reaching its Rayleigh limit which is the maximum charge a liquid droplet can carry. The electrostatic repulsion of similar charges becomes more powerful than the surface tension holding a droplet together. When this phenomenon occurs, Coulombic fission occurs whereby the original droplet 'explodes' creating many smaller, more stable droplets which then reoccurs numerous times. 
Currently, there are two major theories that can highlight the final production of gas-phase ions the ion evaporation model (IEM) and the charge residue model (CRM) (Figure 2.2).

\section{Ion Evaporation Model}
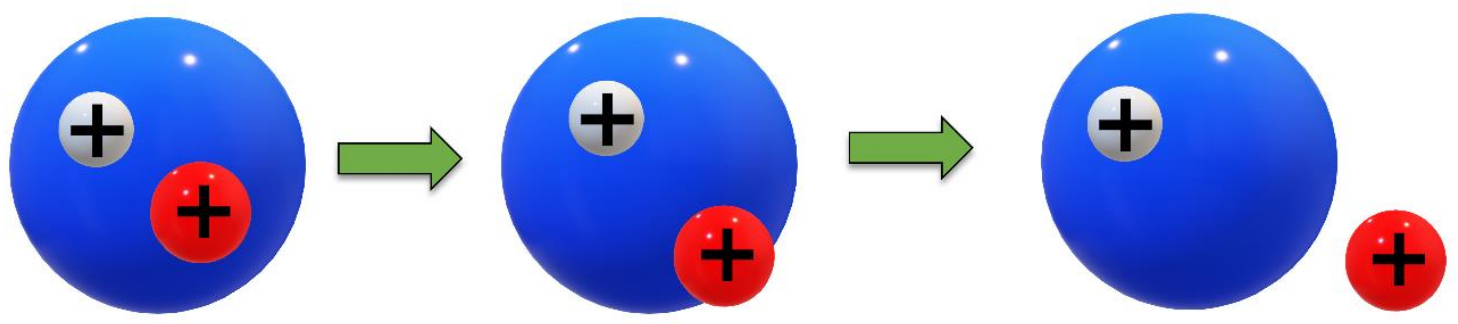

\section{Charge Residue Model}

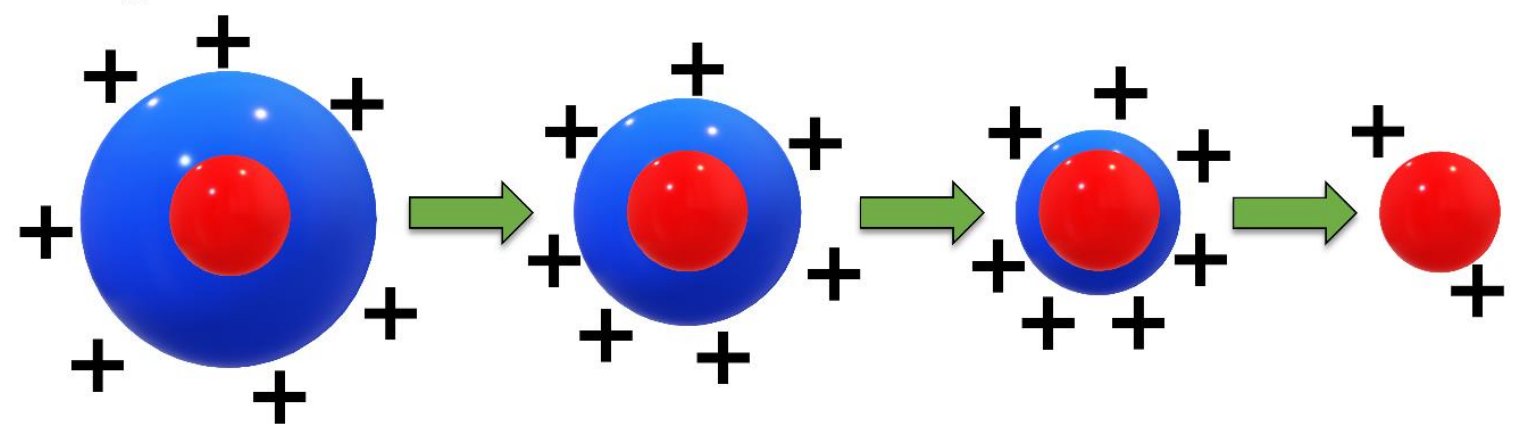

Figure 2.2: Representation of the two established models of electrospray ionization - ion evaporation and charge residue models. The IEM (top) describes ions (red) as they evaporate from a solvent sphere (blue). The CRM (bottom) describes a large biomolecule (red) having the solvent sphere evaporating around it causing multiply charged sites. Figure adapted from reference [11]. Reproduced with permissions from [11]. J.H. Gross, Mass spectrometry, $2^{\text {nd }}$ ed., Springer-Verlag Berlin Heidelberg, 2011. https://doi.org/10.1007/978-3-642-10711-5_4.

The IEM suggests that as a droplet reaches a certain radius, the field strength at the surface

of the droplet becomes large enough to assist the field desorption of solvated ions [13]. The CRM suggests that electrospray droplets undergo evaporation and fission cycles, eventually leading the resulting droplets that contain on average one analyte ion or less [13]. The gas-phase ions form after the remaining solvent molecules evaporates, leaving the analyte with the charges that the droplet carried. The ions observed can exist in many forms such as molecular ions with hydrogen $[\mathrm{M}+\mathrm{H}]^{+}$or another salt adducts such as a sodium $[\mathrm{M}+\mathrm{Na}]^{+}$or potassium $[\mathrm{M}+\mathrm{K}]^{+}$. For negative 
mode, the removal of a hydrogens can be observed $[\mathrm{M}-\mathrm{H}]^{-}$. For larger proteins and biomolecules, there can exist multiple charges in various sites on said species yielding $[\mathrm{M}+\mathrm{nH}]^{\mathrm{n}+}$.

Current evidence illustrates that small molecules are liberated into the gas phase through the IEM, while larger ions (usually folded proteins or biomolecules) form by CRM [14,15]. Recently, a third model combines the charged residue-field emission has been proposed for polymers or unfolded proteins - called the chain-ejection model (CEM) (Figure 2.3) [14,15]. For large macromolecules, there can be many charge states, resulting in a characteristic charge state envelope. All these are even-electron ion species: electrons (alone) are not added or removed, unlike in some other ionization sources.

\section{Chain Ejection Model}

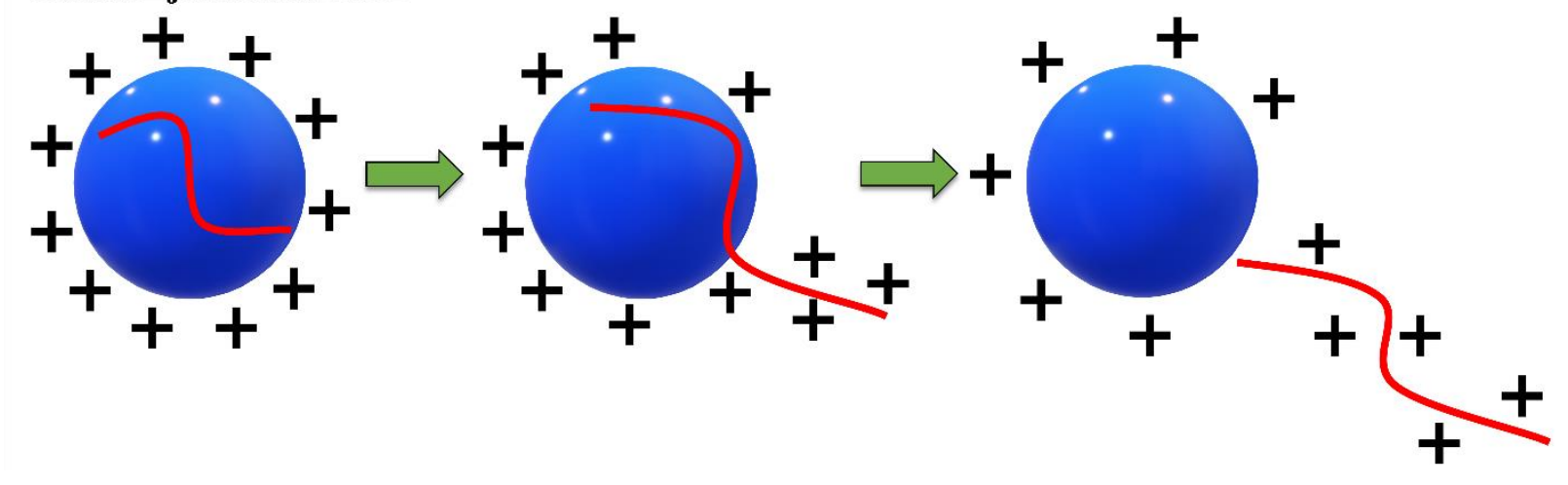

Figure 2.3: Recently proposed chain-ejection model by Konermann group modeling a large biomolecule stretching a leaving the solvent sphere (figure adapted from references $[14,15]$ ).

The efficiency of generating the gas phase ions ESI varies depending on the compound structure, the solvents used, and instrumental parameters. Liquid chromatography couples well with electrospray ionization because they both preserve the native state of proteins as well as other species which can then be analyzed by mass analyzers. Electrospray ionization is also utilized in studying noncovalent gas phase interactions. The electrospray process is thought to be capable of transferring liquid-phase noncovalent complexes into the gas phase without disrupting the 
noncovalent interaction. Just like LC, ESI can be coupled to a variety of mass analyzers to provide information from a variety of instrumentation methodologies.

\subsubsection{Triple Quadrupole Mass Analyzer ( $Q Q Q$ or $Q q Q$ )}

The quadrupole design is simplistic in nature, in that, four rods are arranged in a symmetric array where opposite rods are connected electrically (Figure 2.4).

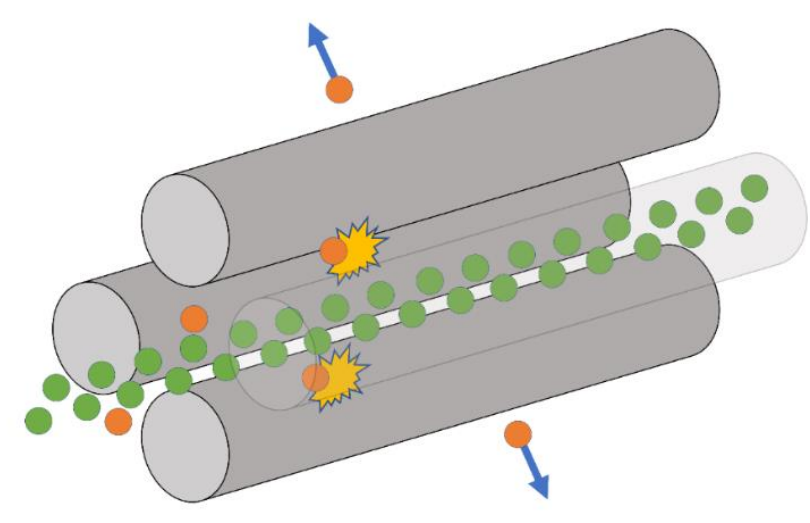

Figure 2.4: Quadrupole mass filter representing ions following the set trajectory (green) and being ejected (orange).

Essentially, a triple quadrupole (QQQ) mass spectrometer operates under the same principle as the single quadrupole mass analyzer with additional sets of mass filters $\left(\mathrm{Q}_{1}\right.$ and $\left.\mathrm{Q}_{3}\right)$ and a collision cell. Both $\mathrm{Q}_{1}$ and $\mathrm{Q}_{3}$ are controlled by direct current (DC) and radio frequency (RF) potentials, while the collision cell, $\left(\mathrm{Q}_{2}\right)$, is only subjected to RF potential. The RF potential associated with the collision cell allows all ions that were selected to pass through it. In some instruments, the normal quadrupole collision cell has been replaced by hexapole or octopole collision cells which improve the filtering efficiency. The QQQ follows the tandem-in-space arrangement, starting with an ambient electrospray ionization, primary mass selection $\left(\mathrm{Q}_{1}\right)$, collision induced dissociation $(\mathrm{CID})\left(\mathrm{Q}_{2}\right)$, mass analysis of fragments produced during $\mathrm{CID}\left(\mathrm{Q}_{3}\right)$, and detection occurring in separate segments of the instrument (Figure 2.5). 


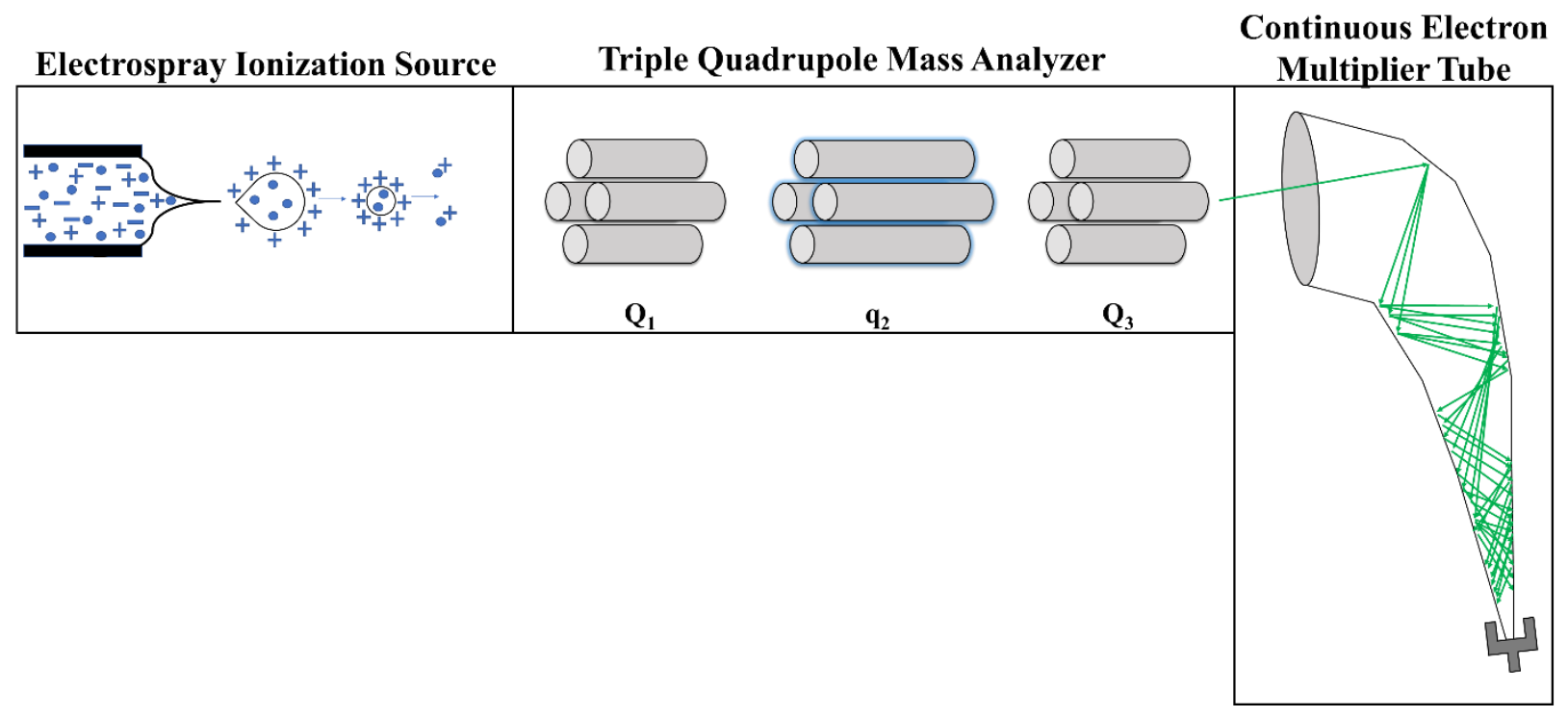

Figure 2.5: Schematic of QQQ with ESI source with a continuous multiplier tube.

As a selected ion enters the quadrupole assembly in the z-direction, an attractive force is exerted by one of the rods with its charge opposite to the ionic charge [11]. Both direct current (DC) and radio frequency (RF) guide specific $\mathrm{m} / \mathrm{z}$ values through the vacuum chamber and analytes that do not possess a stable trajectory collide with the rods and never reach the detector. These trajectory paths of ions can be derived from the Mathieu equations which is calculated by Equation 6:

$$
\frac{m}{z}=K \frac{V}{r^{2} \omega^{2}}
$$

Where $\mathrm{m} / \mathrm{z}$ represents the mass-to-charge ratio of the ion, $K$ is a constant, $V$ is the voltage applied, $r$ is the effective distance between the electrodes, and $\omega$ is the oscillation frequency [11]. Hence, various ions can be "filtered" by exploiting these trajectories for deconvoluting data. The ions entering the system possess unique properties and follow this equation regardless of its initial velocity or position. Once they enter the analyzer, ions have varying stability regions which is represented by Figure 2.6. 


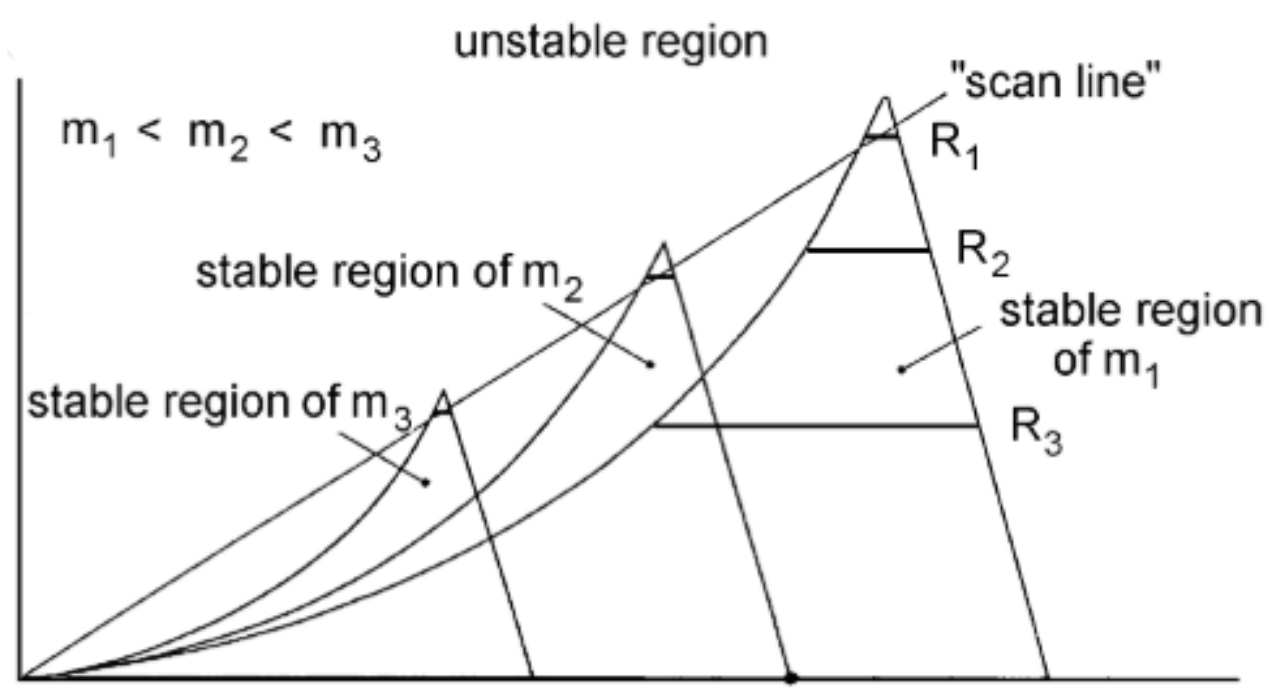

Figure 2.6: Mathieu stability diagram for the stable regions for ions. The region of stability is different for ions with masses $m_{1}, m_{2}$ and $m_{3}$. If the voltage is varied while keeping the ratio between the direct current voltage (y-axis) and high-frequency alternating current voltage (x-axis) constant, a straight scan line 1 is obtained. This scan line passes through respective regions of stability for ions with masses $\mathrm{m}_{1}, \mathrm{~m}_{2}$ and $\mathrm{m}_{3}$. Reproduced with permissions from [11]. J.H. Gross, Mass spectrometry, $2^{\text {nd }}$ ed., Springer-Verlag Berlin Heidelberg, 2011. https://doi.org/10.1007/9783-642-10711-5_4.

The main differences between a single mass filter versus a triple quadrupole filter, is that a quadrupole is limited to the monitoring of fragment ions produced by an ionization source. A single quadrupole filter can view a narrow window of ions using single/selected ion monitoring (SIM). However, the advancement of QQQ systems have led to the implementation of and shift from single ion monitoring to multiple reaction monitoring (MRM) of a triple quadrupole system. The MRM differs from SIM by observing an ion through a single fixed mass window, whereas MRM scans rapidly over multiple mass windows. Hence, MRM acquires traces of multiple fragment ion masses in parallel and is essentially the application of SIM to multiple product ions from one or more precursor ions. This use of multiple product ions has granted MRMs a significant advantage in selectivity and sensitivity. 


\subsubsection{High-Resolution mass spectrometry-Orbitrap}

In terms of mass accuracy, a triple quadrupole mass analyzer provides low to medium resolution ( 10 ppm) for analytes whereas instrumentation methods like the orbitrap provides 1-5 ppm resolution. Orbitrap typically utilizes ESI because of its ability to preserve the natural state of the species under investigation. Unlike the QQQ which forces ions to follow a more linear trajectory, the orbitrap keeps ions in an oscillating movement around the trap represented by

\section{Figure 2.7.}

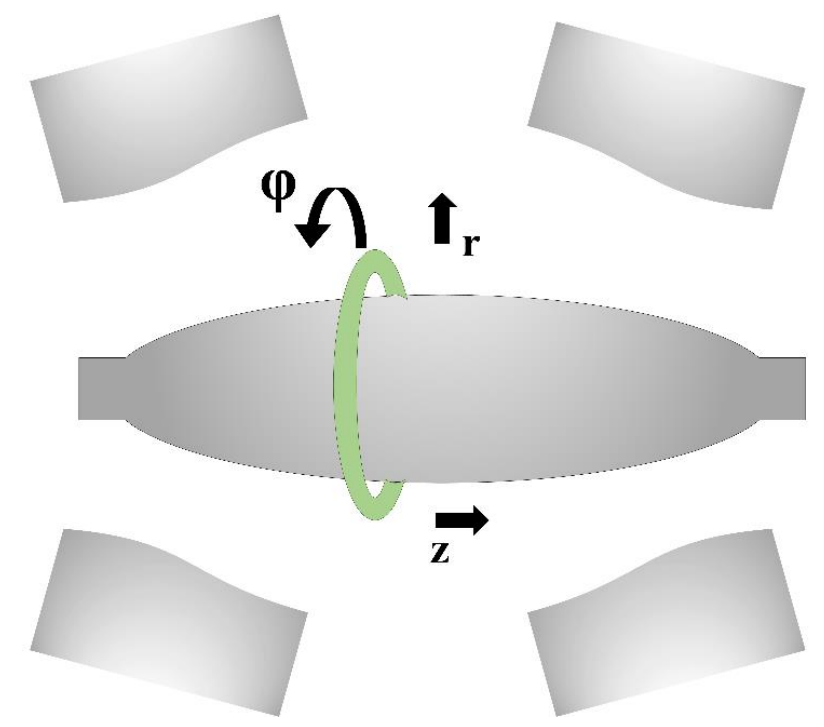

Figure 2.7: Simple schematic of orbitrap mass analyzer with oscillating frequency where ions are moving in spirals around a spindle-like-shaped central electrode. The terms $r$ and $\mathrm{z}$ are cylindrical coordinates ( $\mathrm{z}=0$ being the plane of symmetry of the field). Figure adapted from [11]. J.H. Gross, Mass spectrometry, $2^{\text {nd }}$ ed., Springer-Verlag Berlin Heidelberg, 2011. https://doi.org/10.1007/9783-642-10711-5_4.

The different setup of orbitrap systems utilize a series quadrupole and octapole focusing lenses to filter unwanted species. Ions enter the radiofrequency (RF)-only bent quadrupole of the C-trap and get stored there as they lose energy in gentle collisions with the bath gas [11]. The RF voltage is then ramped down and a high-voltage pulse is applied across the trap, ejecting ions orthogonally to its curved axis. Ion packets enter the Orbitrap analyzer at an offset from its equator and experience strong radial and axial fields. The axial component of the field forces axial 
acceleration and hence so-called excitation by injection, while the radial component sets ions on a circular orbit around the central electrode. As the voltage on this electrode increases during the injection process, the radius of ion packet rotation gets squeezed down. Because of the strong dependence of the rotational frequencies on the ion energies, angles, and initial positions, each ion packet soon spreads over the angular coordinate and forms a thin rotating ring. After voltages are stabilized, the differential amplifier detects a current induced by these rings on the split outer electrodes of the trap. Orbitraps can employ different fragmentation energies which can produce unique product ions for furthering the detection and confirmation of unknown and novel analytes.

\subsection{Overcoming challenges for metal analysis in LC-MS/MS}

As stated previously, LC is a separation technique which works by altering the polarity of the mobile phases and column chemistry. Typically, small organic molecules and proteins are the primary analytes studied, however, metal species can be monitored by coupling to techniques like ICP-MS or ICP-OES $[16,17]$. Further separation of these analytes can be achieved by utilizing specific metallic-binding molecules known as chelating agents. The term chelation refers to the bonding of metal ions to form two or more separate coordinate bonds between a polydentate ligand and a single central atom $[18,19]$. Some examples of chelating agents are ethylenediaminetetraacetic acid (EDTA), dimercaptosuccinic acid (DMSA), and tartaric acid (TT) which have been employed for various application such as pharmaceutical, water treatment, and supplement delivery. Depending on the metal species, these agents can orient themselves into different conformations which can then be elucidated further using the various column strategies mentioned previously [20-22]. However, these strategies are sensitive to $\mathrm{pH}$ changes and can alter the unique isotopic signatures that metal ions yield which can be difficult to monitor and control 
without an ICP-type instrument. Thus, host-guest chemical strategies such as macrocycles have been employed to preserve these natural isotopic ratios.

\subsubsection{Macrocycles - Crown ethers}

Macrocycles are a subclass of supramolecules which are defined by IUPAC "a cyclic macromolecule or a macromolecular cyclic portion of a molecule." One subclass of macrocycles are cryptates which may possess various heteroatoms or other functional groups to increase the effectiveness of interacting with a guest molecule or species. One of the most studied macrocycles, crown ether, was discovered in 1967 by Pedersen [23,24] who reported on their synthesis from glycols and effectiveness to complex with metals. Because of these initial observations, crown ethers have been utilized in various disciplines, such as industrial, medicinal, chemical, and, more recently, forensics $[24,25]$. These ionophores have the unique ability to self-assemble and associate with a broad range of analytes, or guests, for simple molecular recognition, transportation, and extraction.

Initially, the formation of these host-guest systems, it was thought that the "lock-and-key" principle described the mechanism of the guest species with the crown ether. Even though this idea can be applied for more rigid interactions such as proteins with potential drug molecules, other mechanisms have been proposed to provide more insight to this specific host-guest (H-G) interaction. The main principle believed for this specific $\mathrm{H}-\mathrm{G}$ system is noncovalent, electrostatic interactions.

Opposite of covalent interactions, both molecules involved in noncovalent interactions keep their respective electrons and are not shared [26]. Another interesting aspect with this phenomenon is that it allows molecules to undergo various changes in conformation, which provides specific conditions for substances that bind and react with each other. For crown ethers, the oxygen donor atoms bind the alkali metal cation which have a high tendency to form noncovalent bonds. 
Furthermore, noncovalent interactions encompass additional electrostatic interactions which refer to electron-rich (oxygen) or -deficient (metal cations) atoms or ions. By substituting the donor atoms within the cyclic structure, different binding affinities of metal ions can be achieved. Hence, the adaptability of crown ethers has led to multiple strategies to further investigate and understand the relationship between hosts and guests.

\subsection{Strategies for investigating interactions of host-guest chemistry}

\subsubsection{Experimental approaches}

A popular approach to monitor host-guest interactions is utilizing ESI source coupled to mass analyzers as this soft ionization preserves the integrity of the complex and desolvates the solvent media $[27,28]$. The main interests have focused on binding affinity and selectivity when in the presence of multiple ionic species with variable charge states or sizes. For these experimental designs, a set concentration was created for specified metallic species measured based on the relative intensities both separately and in a mixture [29-33]. It was discussed that size (both the inner cavity and cation) is a relevant factor as crown ethers can form "sandwich" structures which is a 2:1 H-G ratio depicted in Figure 2.8. However, these structures appear most often when a species of interest extends beyond the flexibility of the host [31]. 


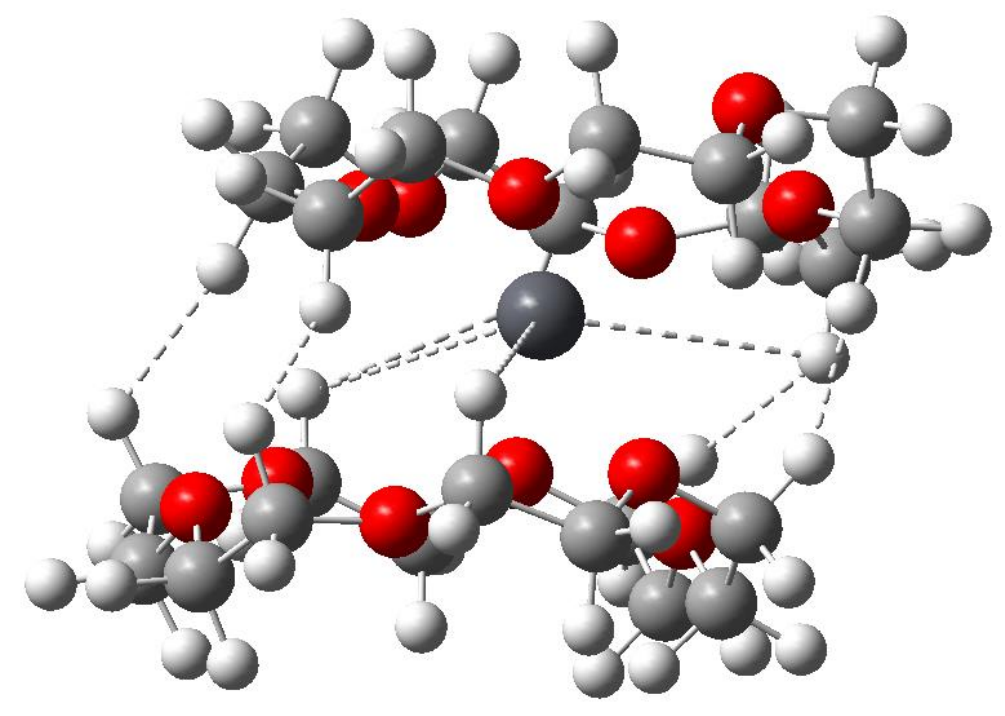

Figure 2.8: Example of a "sandwich" structure with metal ion.

Experimental methods utilizing both low- and high-resolution mass analyzers have given scientists a general concept and insights to help explain this $\mathrm{H}-\mathrm{G}$ phenomenon. The collective response has concluded that crown ethers interact with guests by the noncovalent, electrostatic interactions [24,34-36]. However, technological advances have provided scientists the flexibility to explore structural conformations and to simulate different conditions and scenarios using theoretical calculations.

\subsubsection{Theoretical calculations - Density Functional Theory (DFT)}

There are two types of theoretical methodologies for investigating interactions across variable systems - molecular dynamics and quantum mechanics. Molecular dynamics (MD) modelling observes interactions by employing Newtonian physics and calculated force fields [37,38]. This strategy is less computationally expensive and describes the motions of large biomolecules and proteins more accurately than quantum mechanical approaches. However, MD simulations are less suited for systems where quantum effects are important, i.e., transition metal 
binding [38]. Hence, quantum mechanical (QM) approaches like density functional theory have been used for crown ether complexation with metal ions [18,39-42].

Density-functional theory (DFT) is a computational quantum mechanical modelling method used in physics, chemistry, and materials science to investigate the electronic structure (or nuclear structure) and the ground state of many-body systems, in particular atoms, molecules, and the condensed phases [43]. Using this theory, the properties of a many-electron system can be determined by using functionals, i.e., functions of another function. In the case of DFT, these are functionals of the spatially dependent electron density. DFT is popular due to its ratio between performance and computational cost. It is very fast than other many wavefunction methods, comparatively to other quantum mechanical calculations, while possessing the same level of accuracy and is applied to a variety of fields including condensed-matter physics, computational physics, and computational chemistry.

As usual in many-body electronic structure calculations, the nuclei of the treated molecules or clusters are seen as fixed (the Born-Oppenheimer approximation), generating a static external potential $\mathrm{V}$, in which the electrons are moving $[43,44]$. A stationary electronic state is then described by a wavefunction $\Psi\left(\mathrm{r}_{1}, \ldots, \mathrm{r}_{\mathrm{N}}\right)$ satisfying the many-electron time-independent Schrödinger equation (Equation 2.7)

\section{Equation 2.7}

$$
\widehat{H} \Psi=[\widehat{T}+\widehat{V}+\widehat{U}] \Psi=\left[\sum_{i=1}^{N}\left(-\frac{\hbar^{2}}{2 m_{i}} \nabla_{i}^{2}\right)+\sum_{i=1}^{N} V\left(r_{i}\right)+\sum_{i<j}^{N} U\left(r_{i}, r_{j}\right)\right] \Psi=E \Psi
$$

where, for the N-electron system, $\hat{\mathrm{H}}$ is the Hamiltonian, $\mathrm{E}$ is the total energy, $\hat{\mathrm{T}}$ is the kinetic energy, $\hat{V}$ is the potential energy from the external field due to positively charged nuclei, and $\hat{U}$ is the electron-electron interaction energy. The operators $\hat{T}$ and $\hat{U}$ are called universal operators, as they are the same for any $N$-electron system, while $\hat{V}$ is system-dependent. This complicated 
many-particle equation is not separable into simpler single-particle equations because of the interaction term $\hat{U}$.

DFT utilizes various mathematical functions represented as basis sets which use partial differential equations to represent electronic wave functions. These basis sets can be used to calculate the ground state between molecules and atoms and yields different results including binding energies, emission wavelengths, transition states, and others [45]. Depending on the specific needs of the research and the size of the molecules under investigation, basis sets and even hybrids are used to accurately represent the interactions.

The macrocycles in this study utilize the B3LYP/6-311G++ $(2 \mathrm{~d}, \mathrm{p})$ which describes the valence and core electrons of carbon $(\mathrm{C})$, hydrogen $(\mathrm{H})$, nitrogen $(\mathrm{N})$, oxygen $(\mathrm{O})$, and sulfur $(\mathrm{S})$ atoms. For the metal species, the Los Alamos National Laboratory (LANL2DZ) basis set covers the electrons for the alkali, alkali earth, and transition metals used in this study to measure their thermochemical properties [41,46-50]. The information gathered will address the inability to observe an Sb-18C6 complex as well as the sensitivity of said macrocycle for other common transition metals. This is crucial for further development of heavy metal extractions in wastewater treatment or drug delivery systems.

\subsection{Summary}

The utilization of each of the strategies above (both experimental and theoretical) have been incorporated into this dissertation to further the understanding of not only the abilities of $\mathrm{H}$ G chemistry but also electrostatic interactions. This entity further delves into the practicality and flexibility of liquid chromatography and mass analyzers for monitoring various species and strategies on deconvoluting data. Additionally, it presents an alternative approach to highlight the potential of chemical methods and alterations to by applied to various applications and fields. This 
dissertation starts with a condensed review of the current practices of gunshot residue researchers as well as the current limitations and lack of knowledge. It then addresses these shortcomings by introducing an alternative method and interpretation of GSR information using LC-MS/MS methodology. The previous sections laid the foundational knowledge 


\subsection{References for Chapter 2}

[1] W. Feeney, C. Vander Pyl, S. Bell, T. Trejos, Trends in composition, collection, persistence, and analysis of IGSR and OGSR: A review, Forensic Chem. 19 (2020) 100250. https://doi.org/10.1016/j.forc.2020.100250.

[2] S. Practice, S. Handbook, S. Practice, Standard Practice for Gunshot Residue Analysis by Scanning Electron Microscopy/Energy Dispersive X-Ray Spectrometry, ASTM Int. i (2020) 1-5. https://doi.org/10.1520/E1588-20.

[3] D.A. Skoog, J.F. Holler, S.R. Crouch, Principles of Instrumental Analysis, 7th ed., 2018.

[4] V. D’Atri, A. Murisier, S. Fekete, J.L. Veuthey, D. Guillarme, Current and future trends in reversed-phase liquid chromatography-mass spectrometry of therapeutic proteins, $\operatorname{Tr} \mathrm{AC}$ Trends Anal. Chem. 130 (2020) 115962. https://doi.org/10.1016/j.trac.2020.115962.

[5] G. Purcaro, S. Moret, L. Conte, Hyphenated liquid chromatography-gas chromatography technique: Recent evolution and applications, J. Chromatogr. A. 1255 (2012) 100-111. https://doi.org/10.1016/j.chroma.2012.02.018.

[6] D.J. Winzor, Determination of binding constants by affinity chromatography, J. Chromatogr. A. 1037 (2004) 351-367. https://doi.org/10.1016/j.chroma.2003.11.092.

[7] J.S. Fritz, Ion Chromatography, Anal. Chem. 59 (1987). https://doi.org/10.1021/ac00131a002.

[8] C.A. Lucy, Evolution of ion-exchange: From Moses to the Manhattan Project to Modern Times, J. Chromatogr. A. 1000 (2003) 711-724. https://doi.org/10.1016/S0021-9673(03)00528$\underline{4}$.

[9] B. Buszewski, S. Noga, Hydrophilic interaction liquid chromatography (HILIC)-a powerful separation technique, Anal. Bioanal. Chem. 402 (2012) 231-247. https://doi.org/10.1007/s00216-011-5308-5. 
[10] S. Paul-Dauphin, F. Karaca, T.J. Morgan, M. Millan-Agorio, A.A. Herod, R. Kandiyoti, Probing size exclusion mechanisms of complex hydrocarbon mixtures: The effect of altering eluent compositions, Energy and Fuels. 21 (2007) 3484-3489.

https://doi.org/10.1021/ef700410e.

[11] J.H. Gross, Mass spectrometry, 2nd ed., Springer-Verlag Berlin Heidelberg, 2011. https://doi.org/10.1007/978-3-642-10711-5_4.

[12] M. Yamashita, J.B. Fenn, Electrospray ion source. Another variation on the free-jet theme, J. Phys. Chem. 88 (1984) 4451-4459. https://doi.org/10.1021/j150664a002.

[13] J.H. Gross, Instrumentation, in: Mass Spectrom., 2nd ed., Springer Berlin Heidelberg, Berlin, Heidelberg, 2011: pp. 117-221. https://doi.org/10.1007/978-3-642-10711-5_4.

[14] D.J.D. Pimlott, L. Konermann, Using covalent modifications to distinguish protein electrospray mechanisms: Charged residue model (CRM) vs. chain ejection model (CEM), Int. J. Mass Spectrom. 469 (2021) 116678. https://doi.org/10.1016/j.ijms.2021.116678.

[15] H. Metwally, Q. Duez, L. Konermann, Chain Ejection Model for Electrospray Ionization of Unfolded Proteins: Evidence from Atomistic Simulations and Ion Mobility Spectrometry, Anal. Chem. 90 (2018) 10069-10077. https://doi.org/10.1021/acs.analchem.8b02926.

[16] C.D. Quarles, M. MacKe, B. Michalke, H. Zischka, U. Karst, P. Sullivan, M.P. Field, LC-ICP-MS method for the determination of "extractable copper" in serum, Metallomics. 12 (2020) 1348-1355. https://doi.org/10.1039/d0mt00132e.

[17] E. Matsumoto-Tanibuchi, T. Sugimoto, T. Kawaguchi, N. Sakakibara, M. Yamashita, Determination of inorganic arsenic in seaweed and seafood by LC-ICP-MS: Method validation, J. AOAC Int. 102 (2019) 612-618. https://doi.org/10.5740/jaoacint.18-0148.

[18] N. Yasarawan, K. Thipyapong, Complexation reactions, electronic properties, and 
reactivity descriptors of cysteamine-based ligands in aqueous solution: a PCM/DFT study, Struct. Chem. 29 (2018) 1723-1737. https://doi.org/10.1007/s11224-018-1151-z.

[19] N. Yasarawan, K. Thipyapong, V. Ruangpornvisuti, Substituent effect on the protonrelated phenomena and chelation behavior of hydroxypicolinic compounds: A DFT investigation, Struct. Chem. 27 (2016) 505-524. https://doi.org/10.1007/s11224-015-0579-7. [20] C. Sarzanini, E. Mentasti, Determination and speciation of metals by liquid chromatography, J. Chromatogr. A. 789 (1997) 301-321. https://doi.org/10.1016/S00219673(97)00988-6.

[21] W. Beck, K. Sünkel, Metal Complexes of Indigo and of Some Related Ligands, Zeitschrift Für Anorg. Und Allg. Chemie. (n.d.). https://doi.org/10.1002/zaac.201900363. [22] M. Tella, G.S. Pokrovski, Antimony(III) complexing with O-bearing organic ligands in aqueous solution: An X-ray absorption fine structure spectroscopy and solubility study, Geochim. Cosmochim. Acta. 73 (2009) 268-290. https://doi.org/10.1016/j.gca.2008.10.014.

[23] J.S. Bradshaw, R.M. Izatt, Crown Ethers: The Search for Selective Ion Ligating Agents, Acc. Chem. Res. 30 (1997) 338-345. https://doi.org/10.1021/ar950211m.

[24] I. V. Kolesnichenko, E. V. Anslyn, Practical applications of supramolecular chemistry, Chem. Soc. Rev. 46 (2017) 2385-2390. https://doi.org/10.1039/c7cs00078b.

[25] W. Feeney, K. Menking-Hoggatt, C. Vander Pyl, C.E. Ott, S. Bell, L. Arroyo, T. Trejos, Detection of Organic and Inorganic Gunshot Residues from Hands using Complexing Agents and LC-MS/MS, Anal. Methods. (2021). https://doi.org/10.1039/d1ay00778e.

[26] P.A. Kollman, Noncovalent Interactions, Acc. Chem. Res. 10 (1977) 365-371. https://doi.org/10.1021/ar50118a003.

[27] M. Frańska, A. Michalak, Gas-phase relative stabilities of Tl+-crown ether complexes 
and Rb+-crown ether complexes, Eur. J. Mass Spectrom. 24 (2018) 279-288.

\section{https://doi.org/10.1177/1469066717752794.}

[28] S.M. Blair, J.S. Brodbelt, A.P. Marchand, K.A. Kumar, H.-S. Chong, Evaluation of

Binding Selectivities of Caged Crown Ligands toward Heavy Metals by Electrospray

Ionization/Quadrupole Ion Trap Mass Spectrometry, Anal. Chem. 72 (2000) 2433-2445.

https://doi.org/10.1021/ac991125t.

[29] S.M. Blair, E.C. Kempen, J.S. Brodbelt, Determination of binding selectivities in hostguest complexation by electrospray/quadrupole ion trap mass spectrometry, J. Am. Soc. Mass Spectrom. 9 (1998) 1049-1059. https://doi.org/10.1016/s1044-0305(98)00082-8.

[30] S.M. Blair, J.S. Brodbelt, A.P. Marchand, H.S. Chong, S. Alihodží, Evaluation of alkali metal binding selectivities of caged aza-crown ether ligands by microelectrospray ionization/quadrupole ion trap mass spectrometry, J. Am. Soc. Mass Spectrom. 11 (2000) 884891. https://doi.org/10.1016/S1044-0305(00)00166-5.

[31] C.L. Sherman, J.S. Brodbelt, A.P. Marchand, B. Poola, Electrospray ionization mass spectrometric detection of self-assembly of a crown ether complex directed by $\pi$-stacking interactions, J. Am. Soc. Mass Spectrom. 16 (2005) 1162-1171. https://doi.org/10.1016/j.jasms.2005.03.014.

[32] C.L. Sherman, J.S. Brodbelt, Partitioning Model for Competitive Host-Guest Complexation in ESI-MS, Anal. Chem. 77 (2005) 2512-2523.

https://doi.org/10.1021/ac048518z.

[33] S.M. Williams, J.S. Brodbelt, A.P. Marchand, D. Cal, K. Mlinaric-Majerski, Metal complexation of thiacrown ether macrocycles by electrospray ionization mass spectrometry, Anal. Chem. 74 (2002) 4423-4433. https://doi.org/10.1021/ac011227v. 
[34] H.J. Schneider, A.K. Yatsimirsky, Selectivity in supramolecular host-guest complexes, Chem. Soc. Rev. 37 (2008) 263-277. https://doi.org/10.1039/b612543n.

[35] Y. Tao, R.R. Julian, Factors that influence competitive intermolecular solvation of protonated groups in peptides and proteins in the gas phase, J. Am. Soc. Mass Spectrom. 24 (2013) 1634-1640. https://doi.org/10.1007/s13361-013-0684-z.

[36] A. McKenzie-Coe, S. Bell, F. Fernandez-Lima, Detection of firearm discharge residue from skin swabs using trapped ion mobility spectrometry coupled to mass spectrometry, Anal. Methods. 10 (2018) 4219-4224. https://doi.org/10.1039/c8ay00658j.

[37] D.M. Zuckerman, Statistical physics of biomolecules: An introduction, Stat. Phys. Biomol. An Introd. (2010) 1-325. https://doi.org/10.1201/b18849.

[38] S.B. Choi, B.K. Yap, Y.S. Choong, H. Wahab, Molecular dynamics simulations in drug discovery, Encycl. Bioinforma. Comput. Biol. ABC Bioinforma. 1-3 (2018) 652-665. https://doi.org/10.1016/B978-0-12-809633-8.20154-4.

[39] S. Hadisaputra, H.D. Pranowo, R. Armunanto, Extraction of strontium(ii) by crown ether: Insights from density functional calculation, Indones. J. Chem. 12 (2012) 207-216. https://doi.org/10.22146/ijc.21332.

[40] B. Çiçek, A. Yildiz, Synthesis, metal ion complexation and computational studies of thio oxocrown ethers, Molecules. 16 (2011) 8670-8683. https://doi.org/10.3390/molecules16108670. [41] Z. Jing, G. Wang, Y. Zhou, D. Pang, F. Zhu, H. Liu, Selectivity of 18-crown-6 ether to alkali ions by density functional theory and molecular dynamics simulation, J. Mol. Liq. 311 (2020). https://doi.org/10.1016/j.molliq.2020.113305.

[42] A.A. Bagatur'yants, A.Y. Freidzon, M. V Alfimov, E.J. Baerends, J.A.K. Howard, L.G. Kuz'mina, DFT calculations on the electronic and geometrical structure of 18-crown-6 
complexes with $\mathrm{Ag}+, \mathrm{Hg} 2+, \mathrm{Ag}-0, \mathrm{Hg}+,(\mathrm{HgAgNO})-\mathrm{Ag}-0$, and $\mathrm{HgX} 2(\mathrm{X}=\mathrm{Cl}, \mathrm{Br}$, and I), J. Mol. Struct. 588 (2002) 55-69. https://doi.org/10.1016/s0166-1280(02)00119-7.

[43] J.F. Stanton, A Chemist's Guide to Density Functional Theory By Wolfram Koch

(German Chemical Society, Frankfurt am Main) and Max C. Holthausen (Humbolt University Berlin). Wiley-VCH: Weinheim. 2000. ISBN 3-527-29918-1, 2001. https://doi.org/10.1021/ja004799q.

[44] K. Capelle, A bird's-eye view of density-functional theory, Brazilian J. Phys. 36 (2006) 1318-1341. https://doi.org/10.1590/s0103-97332006000700035.

[45] K.A. Baseden, J.W. Tye, Introduction to density functional theory: Calculations by hand on the helium atom, J. Chem. Educ. 91 (2014) 2116-2123. https://doi.org/10.1021/ed5004788.

[46] R. Puchta, R. Van Eldik, Host-guest complexes of oligopyridine cryptands: Prediction of ion selectivity by quantum chemical calculations, Eur. J. Inorg. Chem. (2007) 1120-1127. https://doi.org/10.1002/ejic.200600715.

[47] Yahmin, H.D. Pranowo, R. Armunanto, Theoretical study on 15-crown-5 complex with some metal cations, Indones. J. Chem. 12 (2012) 135-140. https://doi.org/10.22146/ijc.21353. [48] Y. Zhang, X.Y. Wang, B.H. Luo, Y. Xia, DFT study of crown ether-bridged Z-stilbenes and their complexes with alkali metal cations, J. Organomet. Chem. 699 (2012) 31-38. https://doi.org/10.1016/j.jorganchem.2011.10.032.

[49] A. Üngördü, N. Tezer, DFT study on metal-mediated uracil base pair complexes, J. Saudi Chem. Soc. 21 (2017) 837-844. https://doi.org/10.1016/j.jscs.2017.04.003.

[50] N. Islam, S.S. Chimni, Binding and selectivity of phenazino-18-crown-6-ether with alkali, alkaline earth and toxic metal species: A DFT study, J. Mol. Struct. (2017). https://doi.org/10.1016/j.molstruc.2016.10.100. 


\section{CHAPTER 3}

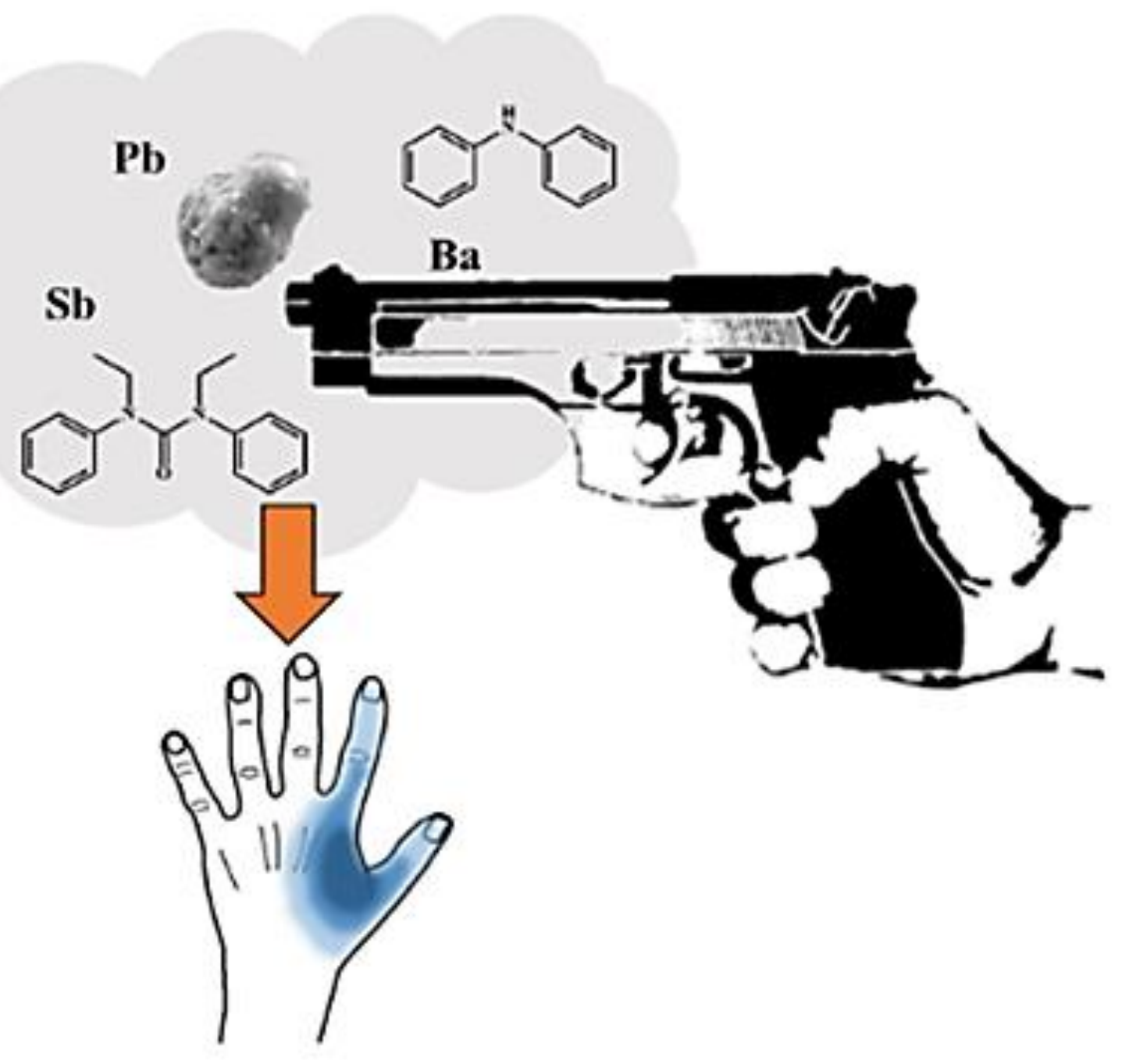

Trends in Composition, Collection, Persistence, and Analysis of IGSR and OGSR: A Review 
Overview: Presented is a review of scientific literature from the past two decades on the various aspects affecting deposition, persistence, and collection of organic and inorganic gunshot residue (OGSR and IGSR, respectively). With market changes in the manufacture of modern ammunition affecting typical elemental composition, morphology of particles, and complex transfer and persistence mechanisms, the forensic community is investigating means to use a more comprehensive approach that incorporates IGSR and OGSR detection. Once collected, inorganic components are more persistent over time than OGSR. Conversely, exogenous compounds that mimic OGSR markers are less prevalent in the environment than IGSR-like elements. As a result, the combined detection of IGSR and OGSR is expected to decrease the occurrence of false positives and false negatives and bring superior confidence in the interpretation of results. This manuscript also compiles information on over 180 primary IGSR and OGSR components reported in the literature for modern ammunition (standard and non-toxic) and their respective analyses. Also, this review offers a discussion of the capabilities and limitations of novel methods and future opportunities to adopt changes in the management of forensic investigations to enhance the versatility, reliability, and response time in the discipline.

The following chapter is an adaptation of a previously published article (O2020: William Feeney, Courtney Vander Pyl, Suzanne Bell, Tatiana Trejos. Trends in composition, collection, persistence, and analysis of IGSR and OGSR: A review. Forensic Chemistry. Published online May 2020, https://doi.org/10.1016/j.forc.2020.1002508E 


\subsection{Introduction}

With the advancement of instrumentation and understanding of the formation of analytes after a combustion event, firearm discharge residue (FDR) or gunshot residue (GSR) has had an increased interest amongst the forensics community. Until recently, analysts have focused on the primer residues, commonly referred to as inorganic gunshot residue (IGSR) [1,2]. However, this is just one aspect of GSR. The second group of compounds originates from the propellant and lubricant and, in turn, is referred to as organic gunshot residue (OGSR) [3-7]. There have been numerous reviews over the past 25 years that cover analysis, distribution, collection, and instrumentation [1,8-10]. In light of this, this review has two main goals. The first is to compile a comprehensive list of IGSR and OGSR analytes present in "standard" (containing lead, barium, antimony) and "non-standard" (lead-free, non-toxic) ammunition. The second is to collate the information gathered by previous reviews and manuscripts that focus on emerging col- lection techniques, persistence information, and amounts (particle count or concentration) detected. Although analytical methods are essential to address, they are not the primary focus of this review.

Current research reports describe the combined detection of IGSR and OGSR compounds to increase the evidentiary value of suspected samples using single techniques or a combination of orthogonal methods [5,11-16]. For detection of inorganic gunshot residues (IGSR), Scanning Electron Microscopy Energy Dispersive X-Ray Spectroscopy (SEM/EDS) is currently considered the gold standard. The ASTM E1588-17 standard practice utilizes SEM/EDS to classify the inorganic particulates based on their elemental composition and morphological features (shape and size) [2]. Indeed, this capability of SEM/EDS to characterize spheroid morphology and conduct elemental analysis on a single microscopic particle is what makes it a highly reliable method for GSR examination. SEM/EDS recognizes these particulates by bombarding electrons onto the surface. Similarly, although much less common at forensic laboratories, a time-of-flight secondary 
ion mass spectrometer (TOF-SIMS) applies an ion beam (i.e., $\mathrm{Bi}, \mathrm{Ga}$ ) to the surface to generate secondary ions. Both techniques can detect small particle ranges and produce high-resolution images for confirmation. TOF-SIMS offers lower limits of detection and provides mass spectral data that helps to resolve signal overlaps encountered by SEM/EDS.

One of the limitations of SEM/EDS is the time required for processing the automated particle analysis and further conducting the manual review of the data by the expert scientist. For instance, analysis of one sample typically takes between 4 and $10 \mathrm{~h}$, depending on the number, size, composition, and abundance of the IGSR. Considering that a ty- pical case consists of at least four specimens per individual of interest (i.e., palm, back, right, and left hands) plus negative and positive quality controls, the laboratory analysis can quickly become a bottle- neck. With recent scientific advancements of detectors (Silicon Drift Detectors vs. Silicon Lithium Detectors) and brighter sources (i.e., CeB6 vs. W filament) SEM/EDS instruments are becoming smaller and faster. A recent study reports a desktop SEM/EDS able to reduce 30-50\% analysis time while still maintaining the ability to perform non- destructive particle counting and elemental mapping with sensitivity comparable to traditional instruments [17-19].

Recent manuscripts have reported several considerations for the interpretation of elemental composition of IGSR particulates and morphological features because of observed shape irregularities and the risk of false positives due to an individual's environment [16,20-25]. Interpretation of IGSR evidence should be evaluated on a case-by-case basis with an understanding of possible elemental combinations of particulates that could arise from an individual's surroundings. Indeed, the ASTM E5188-17 warns examiners not to consider morphology alone as a criterion for the identification of GSR due to its large variability. For instance, welding, fireworks, electrical, and mechanical career fields have an increased likelihood of possessing 
common IGSR analytes on their person [20-28]. Also, the introduction of non-toxic ammunition brings a series of challenges to SEM/EDS analysis. For instance, some of the compounds are more prevalent in the environment (i.e., $\mathrm{Zn}, \mathrm{Ti}, \mathrm{K}$ ) and the detection of lower atomic number elements requires different optimal settings than those established for typical heavy metals. As a potential solution, few studies have reported the incorporation of Cathodoluminescence and Raman to SEM/EDS instruments for expanded capabilities to identify non-toxic GSR [18,19,29]. As another alternative, several groups advocate monitoring OGSR markers in conjunction with IGSR to reduce the rate of false positives $[5,11,13-16]$.

Other methodologies for IGSR analysis include Neutron Activation Analysis (NAA), Inductively Coupled Plasma-based methods (ICP), and Laser-Induced Breakdown Spectroscopy (LIBS), which can provide similar elemental information as SEM/EDS [13,30-36]. However, unlike SEM/EDS, these methods don't have the ability for single-particle analysis.

The effort to increase confidence with GSR information generates emerging techniques that can lead to fast analysis times and minimal sample preparation. For instance, LA-ICP/MS and LIBS take minutes, whereas traditional SEM/EDS lasts several hours for a single sample [11,37] and therefore are attractive methods that could complement current practice. These two techniques employ pulsed laser beams for direct qualitative and quantitative results for increased selectively and simultaneous multi-elemental detection in the low ppm levels [14,31,32,38-40]. Recent advancements with LIBS also allow for portability and versatility at crime scenes and laboratories [34]. Although laser-based techniques can screen for inorganic gunshot residue elements, standard confirmation by SEM/EDS is still required for positively identifying IGSR particles. The potential value of these methods, however, is its utility to make informed decisions at the scene and at the laboratory that can lead to more effective case management. The speed of analysis can also open 
opportunities for more holistic case interpretation by adding alternative specimens that otherwise would be tedious with conventional methods, such as clothing, vehicle surfaces, face areas. A recent study demonstrated the ability of LIBS to provide spatial information along with simultaneous detection of multiple markers (i.e., $\mathrm{Pb}, \mathrm{Ba}$, and $\mathrm{Sb}$ ) from the same location ( $50-100$ $\mu \mathrm{m})$. This ablation approach increases the confidence in the results that the markers come from a particle or group of particles, located in a small area rather than somewhere else within the trace [41]. This spatial information does not match the morphologic information but provides added value over bulk analysis. Although the capability of LIBS and LA- ICP-MS to analyze a single particle has not been explored, it is technically possible, as some configurations allow laser beams as small as $4 \mu \mathrm{m}$. It will be interesting to see if scientists take future studies in this direction. These methods have been combined with different techniques, such as electrochemistry, to compensate for the lack of morpho- logical features [11].

The OGSR detection has been evaluated by diverse methodologies, including chromatographic separation with mass spectrometry detection, Raman spectroscopy, electrochemistry, and ion mobility spectrometry (IMS) [3,6,42-49].

Electrochemical detection employs potential ramps to induce redox reactions at the surface of an electrode. The benefits of this practice are its rapid analysis time (minutes), low production costs, and the ability to detect both IGSR and OGSR analytes. Additionally, it is non-destructive and portable. One obstacle is the inability to detect barium due to its high electrochemical potential. Recently, the combined methods of LIBS and electrochemistry demonstrated high classification rates for IGSR and OGSR [11].

Other quick analysis approaches, such as Raman spectroscopy and FTIR, display their proficiency for complementary information of organic and inorganic compounds [50,51]. Both 
practices implement non- destructive, vibrational frequencies for characterization and imaging of particulates. FTIR provides information for OGSR markers, specifically nitro-compounds such as 2-nitrodiphenylamine (2-NDPA) and 4-nitrodiphenylamine (4-NDPA), but it lacks sensitivity at trace levels [50]. Although fluorescence interferences suppress Raman spectroscopy signals, the scattering and resonance capabilities allow for the dual detection of macro- and micro-sized GSR particles [51]. However, a drawback of both techniques is their inability to detect and identify particles that contain solely metal components (i.e., $\mathrm{Pb}, \mathrm{Sb}, \mathrm{Al}$ ), which are important markers of IGSR for interpretation of evidence.

In OGSR analysis, instrumentation techniques characterize compounds based on molecular properties and formations of product ions. For instance, ion mobility spectrometry (IMS) separates and identifies organic molecules based on their mobility and collisional cross-sections inside a drift tube [42]. Alteration of the drift tube and the ability to couple it with various mass analyzers make it extremely versatile. Typical IMS analysis times can range from the millisecond to subminute timescales and requires minimum resources for sample preparation and operation $[6,42,52,53]$. More traditional OGSR practices such as gas chromatography (GC) and liquid chromatography (LC), rely on volatility and polarity of molecules, respectively. Analysts that apply GC/MS identify analytes by the molecules' Kovats retention index and its probability match scores of the product ions. Mass spectral libraries generate match scores and compare the ratios of formed product ions against a "neat" standard. Scientists that use LC/MS systems manipulate various mobile phase and column conditions to separate similarly structured compounds. The ability to exchange and pair several ionization sources (ESI, APCI, CI, DART) with different mass analyzers (QQQ, TOF, orbitrap) allows LC/MS instrumentation to be adaptable and detect ions in 
the ppb and ppt range. Recently, a study demonstrated the capability to monitor IGSR and OGSR from a single sample [12].

Different reviews highlight the change in interpretation of GSR because of the introduction of "non-toxic" or lead-free ammunition [54- 57]. These modern ammunitions replace traditional primers mixtures of lead, barium, and antimony with bismuth, manganese, aluminum, zinc, titanium, or organic compounds [58-60]. This work compiles lists of both ammunition types and their components while also expanding on information about persistence, collection, and detected amounts illustrated by previous reviews and publications $[1,8,10,61,62]$.

\subsection{Inorganic and Organic Compositions of Modern Ammunitions}

Modifications in modern ammunition broaden the scope of novel IGSR and OGSR markers. Further investigations regarding persistence and prevalence highlight the relevancy of new components as indicators for GSR evidence. Additionally, the capabilities of analytical methods to characterize these diverse analytes are essential to increase the confidence of a collected sample.

The composition of propellants, lubricants, and primers in traditional ammunition contains numerous OGSR compounds with various functions. Explosives in the propellent consist of nitrocellulose (NC), nitroglycerin (NG), and nitroguanidine (NGu), while compounds like ethyl centralite (EC), diphenylamine (DPA), and diphenylamine derivatives act as plasticizers and stabilizers [63]. The purpose of these additives is to prevent thermal decomposition and to lower the viscosity of the ammunition. Post-firing, these analytes adhere to multiple sur- faces such as hands and clothing due to their lipophilic nature.

Traditional primers contain lead styphnate, barium nitrate, and antimony sulfide, which serve as the explosive, the oxidizer, and the fuel, respectively. Other targeted metals and alloys 
originate from the core and jacket of a cartridge or bullet. To lessen the exposure to toxic lead ammunition, manufacturers substitute heavy metals with polymer- coated bullets and lead-free primers. Byproducts from the primer, core, or barrel attach to the environment and can form a dark grey or black ring around a target area known as a "bullet wipe" [64].

Below are two comprehensive lists of traditional and modern ammunition OGSR and IGSR components (Tables 3.1 and 3.2, respectively) frequently reported in the literature. Typical nomenclature, chemical formulae, and functionality of components in various ammunitions are also listed. 


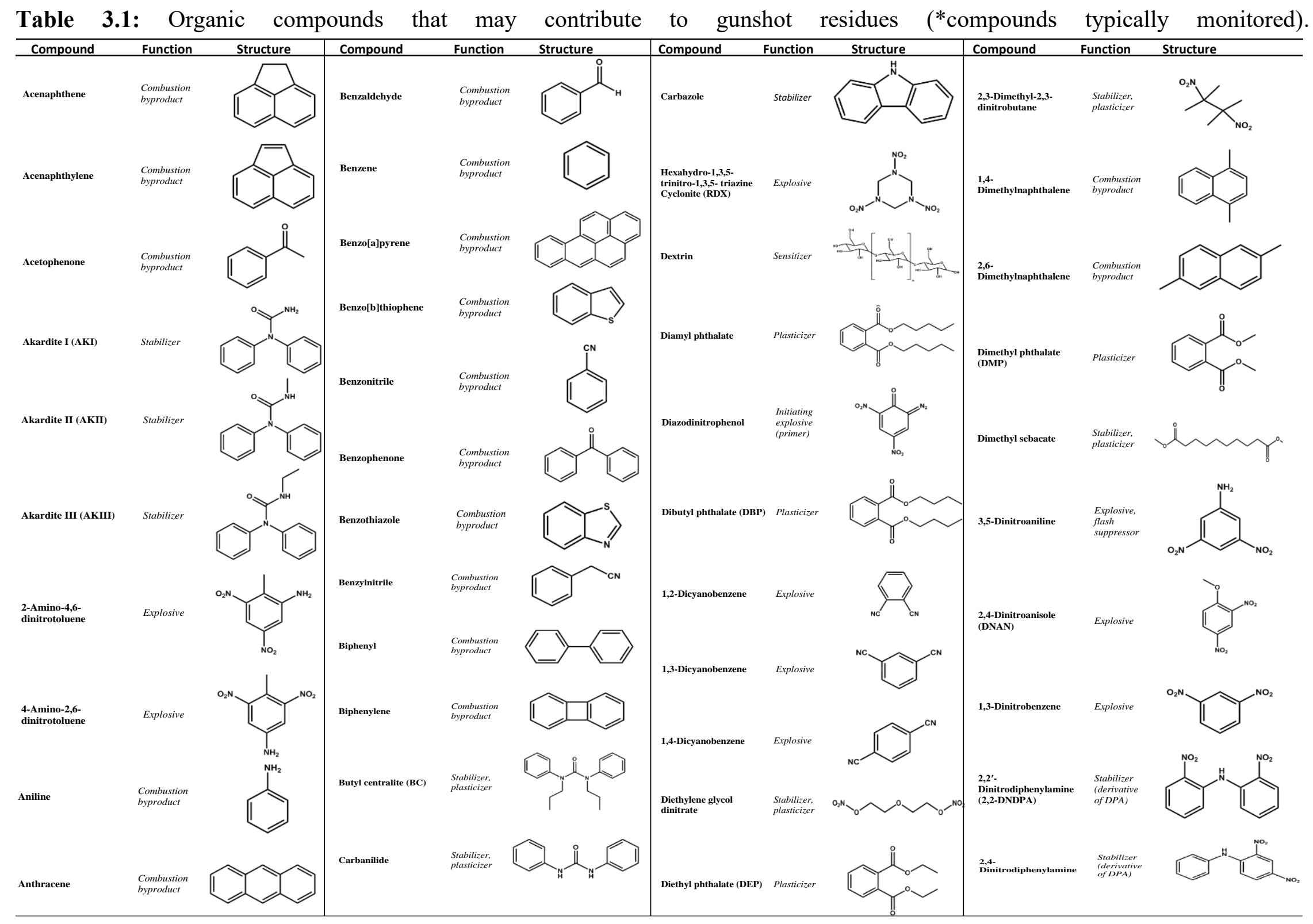




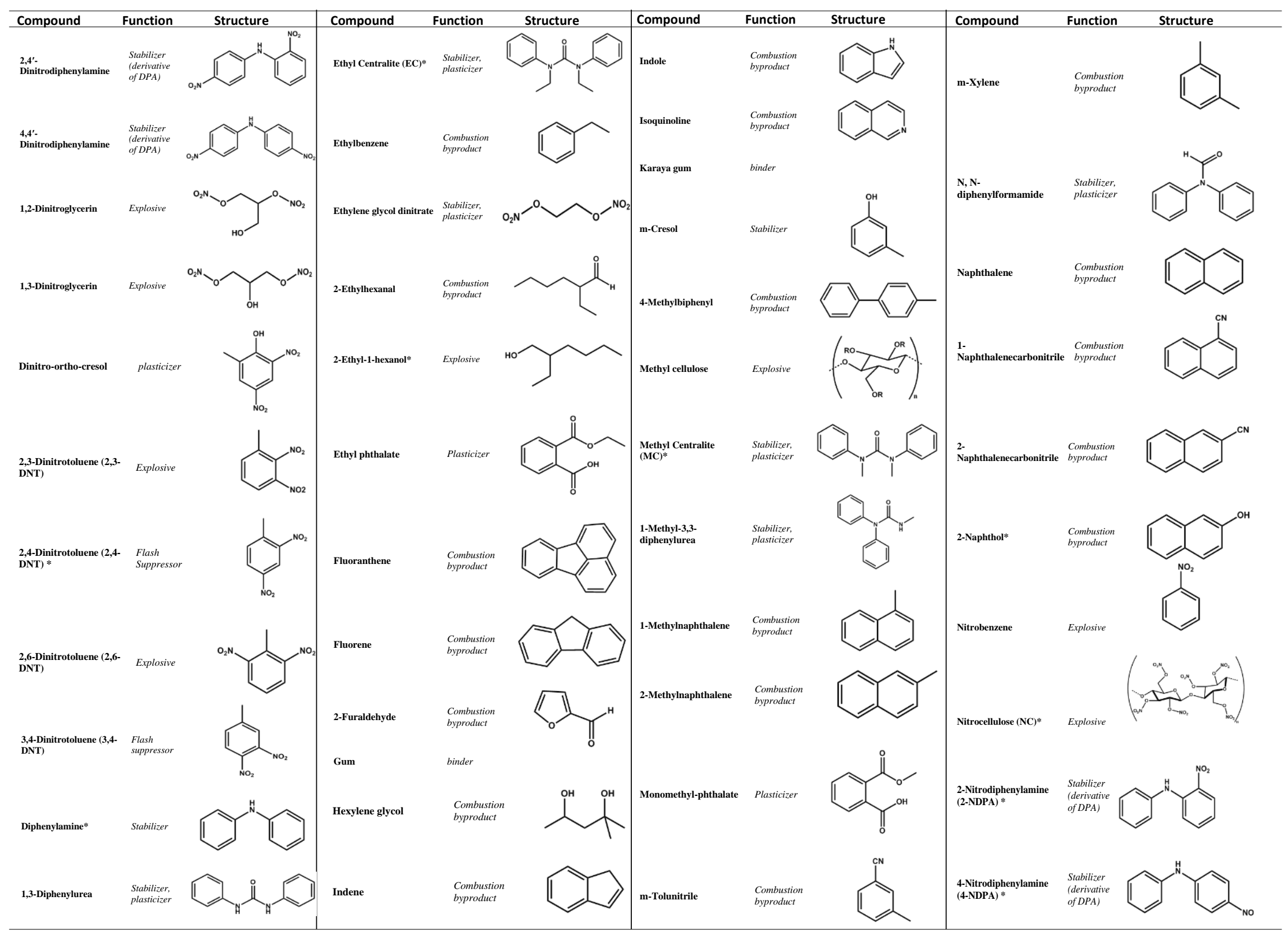




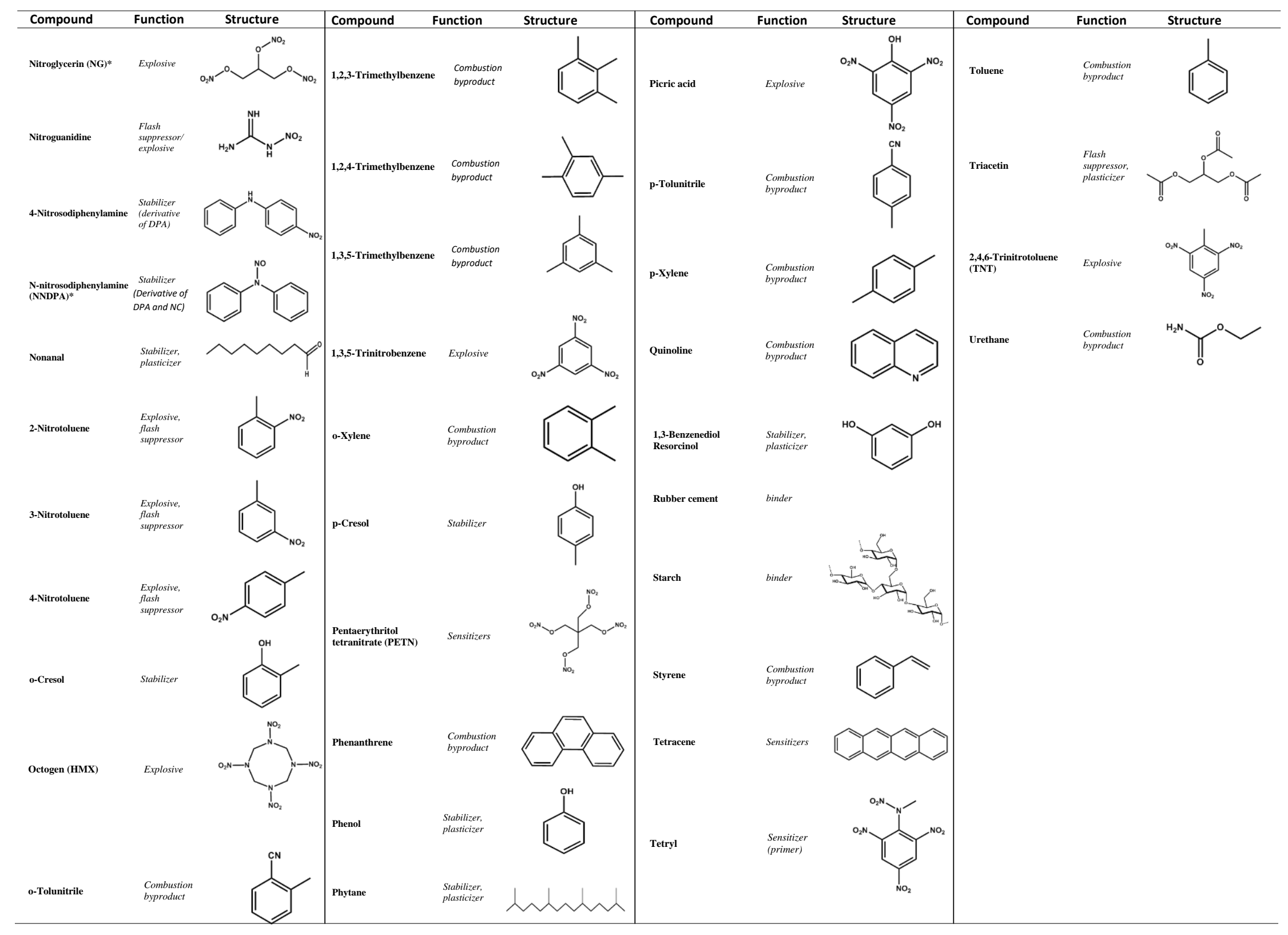


Table 3.2: Inorganic compounds that may contribute to gunshot residue.

\begin{tabular}{|c|c|c|c|}
\hline Compound & Function/Origin & Compound & Function/Origin \\
\hline Aluminum powder* & Reducing agent & Lead peroxide $\left(\mathrm{PbO}_{2}\right)$ & Oxidizer \\
\hline Aluminum oxide* $\left(\mathrm{Al}_{2} \mathrm{O}_{3}\right)$ & Oxidizer & Lead styphnate $\left(\mathrm{PbC}_{6} \mathrm{HN}_{3} \mathrm{O}_{8}\right)$ & Explosive \\
\hline Aluminum sulfide* $\left(\mathrm{Al}_{2} \mathrm{~S}_{3}\right)$ & Fuel & Lead thiocyanate $\left(\mathrm{Pb}(\mathrm{SCN})_{2}\right)$ & Explosive \\
\hline Aluminum silicate* $\left(\mathrm{Al}_{2} \mathrm{SiO}_{5}\right)$ & Stabilizer & Magnesium (Mg) & Reducing agent \\
\hline Antimony (Sb) & Projectile & Manganese* $^{*}$ (Mn) & Fuel \\
\hline Antimony sulfide $\left(\mathbf{S b}_{2} \mathbf{S}\right)$ & Fuel/ friction agent & Manganese oxide* (MnO) & Fuel \\
\hline Antimony sulfite $\left(\mathrm{Sb}_{2}\left(\mathrm{SO}_{3}\right)_{3}\right.$ & Fuel & $\operatorname{Mercury}(\mathrm{Hg})$ & Explosive \\
\hline Antimony trisulfide $\left(\mathbf{S b}_{2} \mathbf{S}_{3}\right)$ & Fuel & Mercury fulminate $\left.\mathrm{Hg}(\mathrm{CNO})_{2}\right)$ & Explosive \\
\hline Barium nitrate $\left(\mathrm{Ba}\left(\mathrm{NO}_{3}\right)_{2}\right)$ & Oxidizer & Molybdenum trioxide* $\left(\mathrm{MoO}_{3}\right)$ & Oxidizer \\
\hline Barium peroxide $\left(\mathrm{BaO}_{2}\right)$ & Oxidizer & Nickle (Ni) & Cartridge case \\
\hline Beryllium (Be) & Fuel & Phosphorous (P) & Cartridge case \\
\hline Bismuth oxide* $\left(\mathrm{Bi}_{2} \mathrm{O}_{3}\right)$ & Oxidizer & Phosphoric acid* $\left(\mathrm{H}_{3} \mathrm{PO}_{4}\right)$ & Oxidizer \\
\hline Bismuth sulfide* $\left(\mathrm{Bi}_{2} \mathbf{S}_{3}\right)$ & Fuel & Potassium chlorate $\left(\mathrm{KClO}_{4}\right)$ & Oxidizer \\
\hline Boron powder* (B) & Reducing agent & Potassium nitrate* $\left(\mathrm{KNO}_{3}\right)$ & Oxidizer \\
\hline Brass & Cartridge case & Silicon monoxide* $(\mathrm{SiO})$ & Fuel \\
\hline Bronze & Cartridge case & Sodium nitrate $\left(\mathrm{NaNO}_{3}\right)$ & Oxidizer \\
\hline Calcium carbonate* $\mathrm{CaCO3})$ & Oxidizer & Sodium sulfate $\left(\mathrm{Na}_{2} \mathrm{SO}_{4}\right)$ & Stabilizer \\
\hline Calcium silicide $\left(\mathrm{CaSi}_{2}\right)$ & Fuel & Silicon monoxide* $(\mathrm{SiO})$ & Oxidizer \\
\hline Chromium (Cr) & Fuel & Sodium nitrate $\left(\mathrm{NaNO}_{3}\right)$ & Oxidizer \\
\hline Chromium oxide $\left(\mathrm{Cr}_{2} \mathrm{O}_{3}\right)$ & Oxidizer & Stannic oxide* $\left(\mathrm{SnO}_{2}\right)$ & Oxidizer \\
\hline Copper $(\mathbf{C u})$ & Cartridge case & Strontium nitrate $\left(\mathrm{Sr}\left(\mathrm{NO}_{3}\right)_{2}\right.$ & Oxidizer \\
\hline Copper thiocyanate (CuSCN) & Explosive & Sulfur $(S)$ & Fuel \\
\hline Copper (II) azide $\left(\mathrm{Cu}\left(\mathrm{N}_{3}\right)_{2}\right)$ & Explosive & $\operatorname{Tin}(\mathbf{S n})$ & Cartridge case \\
\hline Cupro-nickel & Bullet casing & Titanium powder & Reducing agent \\
\hline Ground Glass* & Friction agent & Tungsten $(W)$ & From bullet \\
\hline Iron $(\mathbf{F e})$ & Projectile & Tungsten trioxide* $\left(\mathrm{WO}_{3}\right)$ & Oxidizer \\
\hline Lead $(\mathbf{P b})$ & Projectile & Zinc $(\mathbf{Z n})$ & Cartridge case \\
\hline Lead azide $\left(\mathrm{Pb}\left(\mathrm{N}_{3}\right)_{2}\right.$ & Explosive & Zinc peroxide $\left(\mathrm{ZnO}_{2}\right)$ & Oxidizer \\
\hline Lead oxide (PbO) & Oxidizer & Zinc sulfide* $(\mathrm{ZnS})$ & Fuel \\
\hline Lead nitrate $\left(\mathrm{Pb}\left(\mathrm{NO}_{3}\right)_{2}\right.$ & Oxidizer & Zirconium powder* $(\mathrm{Zr})$ & Reducing agent \\
\hline
\end{tabular}




\subsection{Common Gunshot Residue Sampling Media and Extractions}

Law enforcement, researchers, and forensic scientists employ numerous substrates to recover GSR for analysis [65-69]. Typical sample areas include hands, exposed skin, hair, and clothing, as shown in Figure 3.1. Factors that affect the collection efficiency of a sampling medium include the stability of OGSR and IGSR components and their deposition location [70-76]. For example, OGSR compounds can permeate in the skin, which presents time constraints for analysts

to monitor and report their findings. Because IGSR particulates do not absorb into the skin, they are more susceptible to environmental conditions. Therefore, ideal substrates must be compatible with various instrumentation techniques and efficiently recover both components despite these challenges.

\subsubsection{Adhesives}

The primary collection substrate used in GSR analysis is a carbon-based adhesive. These adhesives collect both IGSR particulates and OGSR compounds because of their flat, porous surface. The tape is typically attached to an aluminum stub on a capped sample holder to reduce the interaction between the sample and collector, which reduces the risk of possible crosscontamination and sample loss. A collection kit consists of four different stubs, one for each side of each hand (i.e., left-palm, right-palm, left-back, right-back), as shown in Figure 3.1. Other advantages of carbon-based tapes include the possibility for sequential analysis on a single sample starting with non-destructive (i.e., electrochemistry, Raman) or minimally destructive (i.e., LIBS) screening tests and then towards confirmatory methods (i.e., GC/MS, LC/MS, SEM/EDS) $[11,14,16,68,75,77,78]$. Researchers have explored chirurgic and acrylic transparent tapes, which are variations of traditional adhesives [76]. However, the ASTM E1588 method outlines carbon adhesive stubs as the collection substrate due to the requirements of SEM/EDS [2,79]. 


\subsubsection{Cloth}

Another material used for casework and research is cloth or cotton swabs [66]. This method applies a cloth or swab soaked with organic solvent (i.e., isopropanol, acetone) directly on the area interest. By creating an organic extraction, this combinational approach focuses on the OGSR compounds $[16,80]$. The thread count of fibers influences the effectiveness of IGSR capture and can cause additional challenges for analysts. For instance, fibers can shield the particulates, causing signal suppression or even charging effects for SEM/EDS.

\subsubsection{Polymer}

A third substrate used for the collection of GSR is polymers. Polymers typically consist of polydimethylsiloxane (PDMS) or similar silicone-based material and exhibit "adhesive-like" qualities $[12,81]$. This method, like adhesives, requires minimal sample preparation and can amass both components of GSR because of its porous surface [12,82]. However, for IGSR, analysis problems arise from polymers because of degassing in the vacuum chamber of an SEM/EDS. Therefore, this collection technique should be limited to other analytical methods such as LC/MS. 


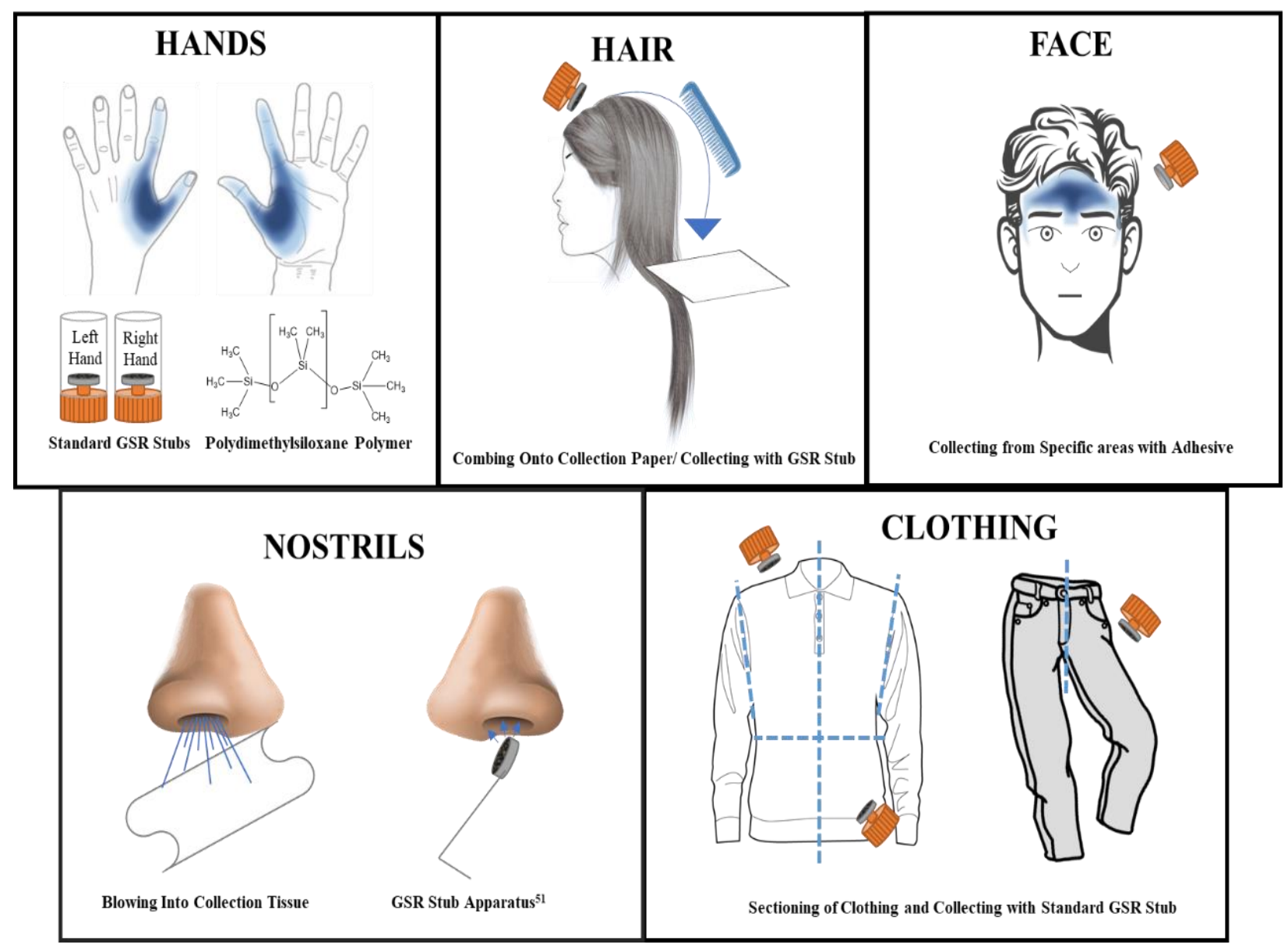

Figure 3.1: Common sampling areas and techniques for gunshot residue evidence collection from hands, hair, face, nostrils, and clothing [51-55].

\subsubsection{Extractions}

After collection, various solvents and mixtures extract analytes from substrates. Non-toxic organic solvents like methanol, acetonitrile, iso- propanol, and aqueous buffers extract OGSR compounds based on the partition coefficient (log P) values [6,85]. These solvents complement techniques such as electrochemistry, GC/MS, and LC/MS [14,44,86-88]. For IGSR analytes, concentrated acid solutions (typically $\mathrm{HNO} 3$ and $\mathrm{HCl}$ ) solubilize the particulates for analysis on different instrumentation techniques such as ICP/MS and LC/MS [14,33,66,89].

For volatile OGSR molecules, scientists use solid-phase micro-extraction (SPME), head space sorptive extraction, and capillary micro- extraction of volatiles (CMV) techniques coupled 
to GC/MS or DART/ MS [14,90,91]. SPME and CMV require an adsorption phase, whether a fiber coated with an affinity phase or a coating inside an open-ended glass capillary, respectively. Both techniques have minimal sample preparation.

\subsection{Deposition and Transfer Trends of Organic and Inorganic Gunshot Residues}

After a discharge event, the vapors escape from several openings of the firearm and begin to cool and coalesce into fine particles containing inorganic and organic compounds associated with GSR $[8,92,93]$. These components originate from the ammunition and firearm and deposit or transfer to other surfaces, including the hands, clothing, and face of the shooter. Additionally, residues are propelled forward through the barrel with the projectile and deposit on a target or site of impact such as walls or windows [94].

However, the detection of GSR on an individual's hands does not indicate that the individual discharged a firearm. Transference from one surface follows four different classifications: primary transfer, secondary transfer, tertiary transfer, and quaternary transfer. Primary transfer occurs after the combustion event where GSR deposits and adheres to surrounding surfaces (i.e., hands, face). Secondary transfer occurs when gunshot residues transfer to another surface by contact with a GSR contaminated surface from a primary transfer. This type of transfer can occur in many ways, such as shaking the hands of the individual who fired the gun, touching the gun after being fired or encountering surfaces that already have GSR on them from the initial firing of the weapon (i.e., tables, walls, floors). Tertiary transfer arises when surfaces contaminated by secondary transfer encounters other surfaces. Finally, quaternary transfer forms from the interaction of one surface with a tertiary transfer sample $[95,96]$.

Several factors influence the number of residues primarily transferred to surfaces, including the type/caliber of the firearm, individual characteristics of the firearm, and the distance 
an individual from a target. For instance, a cylinder may allow for greater deposition of gunshot residues compared to an enclosed chamber (i.e., revolver and pistol, respectively) [1,97]. The cylinder's design allows for vapors to escape easily after discharge, whereas the ejection port on pistols be- comes the essential factor that affects the number of GSR. The location of an ejected cartridge case (i.e., vertically or a particular side) and the duration of the ejection port (opening and remaining open) significantly affects the number of residues that can escape and deposit [97]. The barrel length also plays a role in the amount of GSR deposited on an individual's hands. For example, a longer barrel increases the distance between the plume and a shooter's hands upon discharge, as opposed to a shorter barrel $[93,97,98]$. After firing a rifle, forensic scientists anticipate a large number of residues to be transferred to target materials with fewer residues on hands, while the likelihood of transfer onto the face and clothing increases [98,99].

The number of residues transferred to target materials is reliant on firearm type, as well as the distance from which the individual discharging the firearm is standing (i.e., how far the muzzle of the firearm was from the target). A contact shot often possesses a high amount of GSR localized around the bullet hole entrance. Short-range shooting distances result in a broader spread of gunshot residues on a target with a relatively lower concentration, whereas a long-range shot, shows fewer residues reaching a target. With different types of shootings, a scarce pattern of particles exists that covers the area surrounding the bullet hole [73,100-103]. One recent study shows that IGSR particles can still deposit on surfaces after a projectile passes through other materials or surfaces [99]. To illustrate this, the authors fired one shot through a series of materials, including a glass window, a mannequin, and a sheet of drywall. Replicate test fires were performed and samples from the window, the clothing on the dummy near the bullet entry point, and the area surrounding the bullet hole on the drywall were collected. Out of five test shots under the same 
conditions, the authors found 14-192 characteristics particles on the window, 18-125 particles on the shirt of the dummy, and 4-21 particles on the drywall. This study shows that while the number of detectable residues found decreases as the projectile moves through obstacles, it is possible to find GSR on a final target after impacting other targets (i.e., other victims or building surfaces) [99]. In light of this, several transfer studies specific to the type of gunshot residue (organic vs. inorganic) is presented below.

\subsubsection{Transfer of Organic Gunshot Residue Compounds}

One recent study of OGSR from Hofstetter et al. tested the transfer of residues after the discharge of a $9 \mathrm{~mm}$ on skin, clothing, and in the surrounding environment using IMS-QTOF and UHPLC-QTrap instrumentation. This group analyzed 27 individuals from the general population who do not typically handle firearms, 25 forensic scientists, and the transfer amongst their shooters [54]. They reported that there were no OGSR detected from the general population, and only two scientists had levels of OGSR [54]. Large organic species concentration was located on the hands and forearms of the shooters. Conversely, lower concentrations were detected on the face and hair, while higher concentrations were identified on the clothing of the upper body, highlighting the importance of testing multiple areas of a suspect.

Another study by Gassner et al. studied OGSR secondary transfer in three different scenarios. The first situation simulated the use of an accomplice or innocent bystander that comes and interacts with a discharged firearm. The second scenario included a handshake between the shooter and another individual [104]. A third situation simulated an individual not interacting with a firearm, being arrested by a shooter just after discharge. Throughout this study, the authors were monitoring AK II, N, N-DPF, EC, MC, DPA, N-NDPA, 2-NDPA, and 4-NDPA from two separate ammunition types (Geco and Thun). Most of the compounds in the first scenario were detected, 
except for MC in the Geco ammunition, and AK II in the Thun ammunition, supporting the hypothesis that someone can possess trace amounts of OGSR after handling a firearm. In the second scenario, the non-shooter samples had OGSR compounds detected at least once at the 10ng level except for $\mathrm{N}, \mathrm{N}$-diphenylformamide (N, N-DPF), which was never detected. It should be noted that N, N-DPF while reported by Gassner et al. several times, is not a common marker for OGSR $[54,104,105]$. For the handcuffed non-shooter, the observations in the third simulation were similar to the second simulation. The authors note, however, that if the third scenario required more force in the arrest, the amount of OGSR might increase, concluding that forensic scientists need to be aware that high levels of transfer can occur depending on the firearm used.

\subsubsection{Inorganic Gunshot Residue Particles}

French et al. demonstrated the process of particulate transfer using ultraviolet powder particles through a series of five participants [106]. An individual had particulates deposited on their hand and then proceeded to shake the hand of another person. The handshake recipient then shook the next participant's hand and so on. The study showed that the number of detectable particles drastically decreased as the transfer process continued. The final individual in the series of five only had $0.4 \%-2 \%$ of the number of original particles recovered while the first handshake transferred up to $54 \%$ of particles [106].

French et al. further tested this theory using authentic gunshot residues instead of UV powder $[95,96]$. Three scenarios included a primary transfer onto a shooter, secondary transfer via handshake, and a secondary transfer by firearm exchange. Results showed that primary transfer resulted in the highest particle count by SEM/EDS (206-443 particles), followed by secondary transfer by the handshake (30-129), with the least number of particles transferred during the firearm exchange (14-18). Scenarios testing the effectiveness of secondary transfer through 
firearm handling, handshaking, and clothing handling produced similar results to those reported by French [95].

Merli et al. also investigated the possible transfer of GSR in nose cavities of several individuals, including those who work closely with firearms daily and those who do not handle firearms [56]. Using NAA, individuals who fired more shots showed higher concentrations of Ba and $\mathrm{Sb}$ in their mucus. For instance, an individual who fired 200 shots had $35.045 \mu \mathrm{g}$ and 50.321 $\mu \mathrm{g}$ of $\mathrm{Sb}$ and $\mathrm{Ba}$, respectively. However, the individual who only fired one shot had $0.667 \mu \mathrm{g}$ and $0.908 \mu \mathrm{g}$ of Sb and Ba, respectively [76].

Other studies reported the transfer of GSR to individuals during scenarios where police officers are involved. The high exposure of GSR daily can lead to unknown transfer mechanisms to other individuals, which could lead to samples being flagged as a "positive" even if the individual in question was not related to the firearm incident. Sources of GSR transfer during the detaining of an individual, the arresting process, police vehicles, and detention centers were characterized as possible contamination sites. Charles et al. investigated low contamination and high contamination risks in two different arresting scenarios related to the clothing of an officer [107]. The study compared officers wearing civilian clothing (low-risk) versus wearing technical vests and gloves (high-risk). The low-risk scenario showed a lower number of particles on the arrestee's hands than in the high-risk scenario. The highest contamination during the high-risk scenarios originated from the gloves the police officers with a particle count of 320 [107]. A separate study by Gerard et al. showed the potential of contamination from standard police officer equipment, concluding that roughly $24 \%$ of the sampled police officer equipment ( $\mathrm{n}=24$ sleeves, $\mathrm{n}=18$ batons, $\mathrm{n}=26$ handcuffs) tested positive for gunshot residues [108]. 
Several studies, like Berk et al., tested the likelihood of contamination from various surfaces to a suspect. They investigated the potential contamination to an arrested individual who was loaded into the police car and detained at a police station in Chicago, Illinois and found the vehicles contributed a negligible amount (0-2 particles) [109]. A similar study confirmed this finding, where only one particle was detected from 18 different Toronto, Durham, and York police vehicles [108]. Surprisingly, bars on holding cells and tabletops in the detention facilities were more prone to contamination compared to police official vehicles (20 and 34 particles, respectively) [109]. Ali et al. demonstrated that most surfaces in a Pittsburgh, Pennsylvania police station only contained particles that were commonly associated with GSR, which have compositions consisting of only one of the three characteristic elements: $\mathrm{Pb}, \mathrm{Ba}$ and $\mathrm{Sb}$ [16]. Diaz et al. investigated the amount of airborne gunshot residue particles present in Brazil ballistics laboratory located in Sao Paulo [33]. Filters collecting the lab's air over six days were digested and analyzed using ICP/MS. The levels of $\mathrm{Sb}, \mathrm{Ba}$, and $\mathrm{Pb}$ in the surrounding air did not lead to contamination of the lab personnel [33].

Investigations for other potential contamination sources from police officials' hands to a suspect's person have been conducted. One study testing the hands of police officers after the start of their shifts showed that out of 33 officers, 28 of the samples (85\%) tested positive for GSR particles. Conversely, out of 17 officers, only one officer had a single particle after washing their hands directly after handling their firearm, indicating that hand-washing eliminates almost all contamination [110]. Lucas et al. randomly sampled firearm-handling officers in the South Australia Police and concluded that $7.9 \%$ of all officers sampled $(n=76)$ had at least one characteristic GSR particle on their hands without firing their weapon that day and only one officer had 14 characteristic particles [111]. Twenty-one of the officers then simulated an arrest situation 
after firing two rounds from their firearm, and all 21 arrest situations resulted in the transfer of GSR particles to the person of interest. The number of particles transferred ranged from 2 to 110 particles for the simulation [111].

These studies demonstrate that the detection of GSR on the hands of an individual does not definitively mean a suspect discharged a firearm. These studies raise two necessary precautions: 1) the need for police officers to wash their hands or use disposable gloves before an arrest, and 2) conduct sampling at a scene when possible before bringing the individual inside a police car or to jail. The studies also serve as a precedent that perhaps GSR investigations should be focused or complemented with detection of residues from surfaces that are not as exposed to contact or activity contamination, such as hair and nostrils.

Demonstrated above are several scenarios of secondary transfer where an individual did not discharge a firearm. However, an individual can possess GSR on their hands through primary transfer being near a discharged weapon. French et al., showed that individuals standing one meter behind a shooting incident still had GSR particles primarily transferred to their hands [96]. Out of three test fires, the highest number of particles found on a bystander's hands was 36 characteristic particles. Lindsay et al. conducted a similar study where bystanders were positioned one meter away on multiple sides (right, left, and back) of the shooter. In all incidents, the left and right hands of the individual bystanders were positive for GSR particles [84]. Additional studies also show that individuals close to a shooting incident can have ample amounts of GSR deposited on their hands $[112,113]$.

\subsection{Persistence of Both Organic and Inorganic Components of Gunshot Residue}

Persistence in GSR refers to the duration of OGSR and IGSR components to remain on surfaces after deposition or the time to remove these analytes from surfaces after discharge. Several 
aspects influence this property of the components, such as the surface composition, environmental conditions, and physical and chemical properties of the compounds.

\subsubsection{Organic Components}

For the OGSR components, the chemical composition, and other properties such as lipophilicity and boiling points significantly affect the interaction. Several temporal studies have explored OGSR detection by various instruments such as GC/MS, LC/MS, and IMS. For instance, Arndt et al. investigated N-NDPA, DMP, and DPA and their persistence on the skin after a firing event using IMS [42]. The longevity of the organic compounds was tested after a shooting at specific time intervals (1, 2, 3, 4 hours). The individuals did not wash their hands and concluded that organic molecules are less prone to secondary transfer and could persist on the skin up to four hours via a handheld IMS unit.

Meng and Caddy summarized Lloyd's work where residue molecules, like nitroglycerin, were detected up to seven hours on the face, throat, and hands of an individual. With suicide victims, organic residues could persist on unwashed hands up to 48 hours [61]. However, a contradictory study under the same review, Douse reported no presence of nitroglycerin on hands after 11 test firings. On clothing, nitroglycerin, nitrocellulose, and diphenylamine compounds were detected six hours after firing, leading to the conclusion that clothing is more effective in retaining some organics than hands.

One of the most recent studies involving persistence reported by Maitre et al. [114] tested the persistence of EC, DPA, and N-NDPA. Shooting samples were collected from both hands using carbon adhesive stubs and tested at different time points (30 min, 1, 2, and $3 \mathrm{~h}$ ) and analyzed using an LC-QQQ. In more than 70\% of the samples, OGSR compounds were detected up to four hours after the firing event [114]. Factors influencing their persistence include environmental 
conditions (i.e., airflow) and conditions of the shooter (i.e., skin, hair thickness, clothing). Two different firearms were used, a Glock 221 caliber .40 S\&W and a .357 Magnum (.357 Mag) S\&W revolver model 686 (4” barrel) with Winchester WinClean1 (180 Gr. Brass Enclosed Base) and traditional primer: PPU Ammunition1 (158 Gr. Semi- Jacketed Hollow point), respectively. For both the revolver and the semi-automatic, all the organic compounds' signals decreased to $8 \%$ of the initial peak height after 4 hours, which highlights the necessity of quick and effective collection techniques and storage conditions.

\subsubsection{Inorganic Particles}

For IGSR, authentic hand samples were tested using multiple types of instrumentation such as SEM/EDS, NAA, ICP (MS and OES), and LIBS. Rosenberg and Dockery were one of the first research groups to evaluate the persistence of GSR on an individual's hands after the dis- charge of a firearm using alternative methods such as LIBS [32]. Individuals fired six consecutive rounds of ammunition and collected using 3 M 5490 PTFE tape from the shooters at different periods over a week. GSR elements were still detectable by LIBS up to five days after the initial shooting. Interestingly, this time of persistence is very lengthy and does not agree with other literature values reported [32].

While LIBS is a valuable technique for the detection of GSR, the most notable instrument to study the persistence of residues on an individual's hands is SEM/EDS because of the ASTM E1588 guideline. Jalanti et al. collected a sequence of hand samples from a shooter at different time intervals, including - immediately after firing and two, four, and six hours after firing [115]. The highest number of particles detected in each scenario originated from samples collected immediately after discharging the firearm (160-187 particles). However, in all three scenarios, large numbers of GSR particles (4-111 particles) were still detectable after six hours of regular activity. 
These findings illustrate that particles can be detected after six hours after discharge, and the activity can decrease the number of particles persisting on hands. In turn, several groups have evaluated the relation of activity type to the persistence of these residues [30,115-119].

For instance, Kilty [116] reported that an individual who washed their hands with soap and water and dried their hands on a rough surface (i.e., paper towel) removed all detectable amounts of GSR. However, a person who only briefly washed their hands without soap and wiped their hands on a clothing item did not remove all GSR particles, which indicates the robustness of washing influences the persistence of GSR [116]. Conversely, minimal to no activity after a discharge leads to more substantial retention of GSR, which was studied with suicide victims. The most notable manuscripts compare suicide cases versus simulated suicides; GSR particles are still detected any- where from 2 to 60 days after the shooting incident occurred [30,118-121].

A study by Brozek-Mucha found that particles embedded in the hair and located on the face of an individual persisted longer than samples collected from and individual's hands (over 4 hours) [83]. These results agree with a Zeichner and Levin's studies where GSR particles were still detectable on in hair samples 24 hours after discharge but non-detectable on their hands at the same time of collection $[78,122]$. Both studies demonstrate the need for further persistence studies on various surfaces because the loss of particles on hands occurs faster than the hair or face.

Clothing and different fabric types also play a role in the retention of GSR post-discharge. Charles et al. studied the effect of four different fabric types (i.e., cotton, worn cotton, leather, and wool) and their ability to retain GSR over time [72]. Fabric samples were placed two meters away from the firearm and samples were collected 10 minutes after shooting. Results showed that the recovery of GSR was highest from the leather textile, followed by cotton, worn cotton, and wool. The group also tested the ability of the wool and leather textiles to GSR particles after shaking the 
garment. For both cases, similar numbers of particles were collected off the shaken and non-shaken sample. This study showed that the durability of fabric influences the persistence of GSR on its surface. However, Vinkurov et al. further went on to prove that brushing or washing of clothing targets significantly diminishes the number of particles retained on the item and should be taken into consideration when dealing with such situations [123].

The information provided can be cumbersome and difficult to interpret because of the magnitude of studies on these different factors and methods. Therefore, Table $\mathbf{3 . 3}$ compiles essential studies contributing to the understanding of GSR formation, illustrated collection techniques, developed instrumental methodologies, and reported findings of modern ammunition. The comprehensive list presented below aims to compare method capabilities and limitations under the specific experimental conditions, including firearm type, sampling area, collection medium, storage, and instrumentation. The information was divided into three categories, as per the method's ability to detect OGSR (Table 3.3a), IGSR (Table 3.3b), or both (Table 3.3c). Although a direct comparison of the methods detection limits or the number of particles detected is not possible, due to differences in calculations and reporting of those figures, the information is still useful as an overall assessment of their relative sensitivities. 


\begin{tabular}{|c|c|c|c|c|c|c|c|c|c|}
\hline Author & Firearm & $\begin{array}{c}\text { \# of } \\
\text { Shots } \\
\end{array}$ & $\begin{array}{c}\text { Sampling } \\
\text { Area } \\
\end{array}$ & $\begin{array}{c}\text { Collection } \\
\text { Media } \\
\end{array}$ & Storage & Instrumentation & $\begin{array}{c}\text { Analysis } \\
\text { Time }\end{array}$ & $\begin{array}{c}\text { Compounds } \\
\text { Detected } \\
\end{array}$ & $\begin{array}{c}\text { Method } \\
\text { DL }\end{array}$ \\
\hline $\begin{array}{c}\text { MacCrehan } \\
{[57]}\end{array}$ & $\begin{array}{c}.22 \text { caliber revolver } \\
\text { Winchester } \\
\text { Wildcat or Federal } \\
\text { lightning long- rifle }\end{array}$ & 5 & $\begin{array}{l}\text { Nasal } \\
\text { Mucus }\end{array}$ & Nylon Mesh & NR & $\mathrm{CE}$ & $<15 \min$ & $\begin{array}{c}\text { NG, NB, 2,4-DNT, } \\
\text { 2,6-DNT, 4-NT, } \\
\text { DPA, EC }\end{array}$ & NR \\
\hline $\begin{array}{c}\text { Khandasammy } \\
{[58]}\end{array}$ & CCI Blazer $9 \mathrm{~mm}$ & NR & Cloth & $\begin{array}{c}\text { Carbon } \\
\text { Adhesive } \\
\text { Tape } \\
\end{array}$ & NR & $\begin{array}{l}\text { Raman Imaging } \\
\text { Microscope }\end{array}$ & $<60 \min$ & NR & NR \\
\hline Lopez [59] & $9 \mathrm{~mm}$ Luger & NR & Cloth & $\begin{array}{l}\text { Carbon } \\
\text { Adhesive } \\
\text { Tape }\end{array}$ & NR & $\begin{array}{l}\text { Raman imaging } \\
\text { microscope }\end{array}$ & NR & DPA, NC & NR \\
\hline Lopez [60] & NR & NR & Cloth & $\begin{array}{c}\text { NA } \\
\text { (Analyzed } \\
\text { Cloth) } \\
\end{array}$ & NR & $\begin{array}{l}\text { Raman imaging } \\
\text { microscope }\end{array}$ & NR & $\begin{array}{c}\text { DPA, N-NDPA, 2- } \\
\text { NDPA, 4-NDPA, EC }\end{array}$ & NR \\
\hline Roberts [61] & $\begin{array}{l}25 \text { different } \\
\text { ammunitions }\end{array}$ & NR & NR & NR & NR & GC-MS & $<25 \min$ & NG. 2,4-DNT, DPA & $\begin{array}{c}0.34-1.4 \\
\mathrm{mM}\end{array}$ \\
\hline Arndt [62] & $\begin{array}{c}\text { Glock Model } 199 \\
\text { mm } \\
\text { Browning GP35 Hi } \\
\text { Power } 9 \mathrm{~mm} \\
\end{array}$ & 3 & $\begin{array}{l}\text { Hands } \\
\text { (top and } \\
\text { sides) }\end{array}$ & $\begin{array}{l}\text { Cloth } \\
\text { acetone }\end{array}$ & $\begin{array}{c}4^{\circ} \mathrm{C} \\
\text { freezer }\end{array}$ & IMS & $<1 \mathrm{~min}$ & $\begin{array}{c}\text { DMP, DPA, N- } \\
\text { NDPA, 2,2-DNDPA, } \\
\text { 4,4-DNDPA }\end{array}$ & $10 \mathrm{ng}$ \\
\hline Yeager [63] & NR & NR & Hands & $\begin{array}{l}\text { Cotton } \\
\text { Swabs } \\
\end{array}$ & $-26 \mathrm{C}$ & IMS & $<30 \mathrm{~s}$ & $\mathrm{EC}, \mathrm{MC}, \mathrm{DMT}, \mathrm{DPA}$ & $\begin{array}{c}1-100 \\
n g\end{array}$ \\
\hline Hofstetter [53] & $9 \mathrm{~mm}$ & $1-3$ & $\begin{array}{l}\text { Hands, } \\
\text { Wrists, } \\
\text { Face, hair, } \\
\text { T-shirt, } \\
\text { Gloves, }\end{array}$ & $\begin{array}{l}\text { Carbon } \\
\text { Adhesive } \\
\text { Tape } \\
\text { Swabbing }\end{array}$ & $\begin{array}{c}4^{\circ} \mathrm{C} \\
\text { freezer }\end{array}$ & IMS-QTOF & $9 \mathrm{~min}$. & $\begin{array}{c}\text { AK II, MC, N, N- } \\
\text { DPF, EC, DPA,2- } \\
\text { DPA, 4-NDPA, N- } \\
\text { NDPA }\end{array}$ & $\begin{array}{c}0.005- \\
0.5 \\
\mathrm{ng} / \mathrm{mL}\end{array}$ \\
\hline Tong [64] & gunpowder & NA & NA & NA & NR & GC-TEA & $<10 \min$ & $\begin{array}{c}\text { DPA, N-NDPA, 4- } \\
\text { NDPA, 2-NDPA, } \\
\text { 2,4-2NDPA }\end{array}$ & $\begin{array}{l}0.05-1 \\
n g\end{array}$ \\
\hline Laza $[65]$ & $\begin{array}{c}9 \mathrm{~mm} \text { Para } \\
\text { ammunitions }\end{array}$ & 5 & Hands & $\begin{array}{l}\text { Cotton } \\
\text { Swabs }\end{array}$ & $\begin{array}{l}\text { Stored } \\
\text { freezer }\end{array}$ & LC-MS & $<10 \mathrm{~min}$ & $\begin{array}{c}\text { DPA, AKII, 2- } \\
\text { NDPA, 4-NDPA, EC, } \\
\text { MC, N-NDPA }\end{array}$ & $\begin{array}{l}\text { Low ng } \\
\text { levels }\end{array}$ \\
\hline Perret [66] & NR Explosives & NR & Hands & $\begin{array}{l}\text { Cotton } \\
\text { Swabs }\end{array}$ & NR & LC-QQQ & $<10 \min$ & $\begin{array}{c}\text { NG, TNT, PETN, } \\
\text { RDX }\end{array}$ & $\begin{array}{c}2 \mu g- \\
2710 \mu g\end{array}$ \\
\hline
\end{tabular}




\begin{tabular}{|c|c|c|c|c|c|c|c|c|c|}
\hline Stevens [67] & $\begin{array}{c}\text { Glock } 9 \mathrm{~mm} \\
\text { semiautomatic } \\
\text { pistol } \\
\text { Smith and Wesson } \\
.38 \text { revolver }\end{array}$ & $\begin{array}{c}1-5 \\
\text { shots }\end{array}$ & Hands & $\begin{array}{l}\text { Cotton } \\
\text { Swabs }\end{array}$ & NR & TD-GCMS & $<20 \min$ & $\begin{array}{c}\text { DMP. 2,4-DNT, } \\
\text { DPA, MC, } \\
\text { Carbazole, EC, DBP, } \\
\text { 2-NDPA, 4-NDPA }\end{array}$ & $\begin{array}{c}0.05- \\
500 \mathrm{ng}\end{array}$ \\
\hline Gassner [68] & $\begin{array}{l}\text { 9mm Luger } \\
\text { handguns semi- } \\
\text { auto pistol Sig } \\
\text { Sauer P226 S\&W }\end{array}$ & 3 & $\begin{array}{l}\text { Hands, } \\
\text { Wrists }\end{array}$ & $\begin{array}{l}\text { Carbon } \\
\text { Adhesive } \\
\text { Tape } \\
\text { Swabbing }\end{array}$ & $\begin{array}{c}4^{\circ} \mathrm{C} \\
\text { freezer }\end{array}$ & UHPLC -QTrap & $7 \mathrm{~min}$ & $\begin{array}{c}\text { AK II, MC, N-N- } \\
\text { DPF, EC, DPA, 2- } \\
\text { NDPA, 4-NDPA, N- } \\
\text { NDPA }\end{array}$ & $\begin{array}{c}0.005- \\
0.5 \\
\mathrm{ng} / \mathrm{mL}\end{array}$ \\
\hline Maitre [69] & $\begin{array}{c}\text { Glock } 22, .40 \\
\text { S\&W caliber } \\
\text { WinClean } ® \text { ammo } \\
\text { (180grains) }\end{array}$ & NR & Hands & $\begin{array}{l}\text { Carbon } \\
\text { Adhesive } \\
\text { Tape } \\
\text { Swabbing }\end{array}$ & $\begin{array}{l}\text { Stored } \\
\text { at } 4^{\circ} \mathrm{C} \\
\text { freezer }\end{array}$ & UPLC MS/MS & $27 \mathrm{~min}$ & EC, DPA, N-NDPA & NR \\
\hline Maitre [70] & $\begin{array}{c}\text { Glock } 22 \text { caliber } \\
.40 \mathrm{~S} \& W \\
.357 \text { Magnum } \\
\text { (.357 Mag) S\&W } \\
\text { Revolver model } \\
686 \text { (4" barrel) } \\
\end{array}$ & NR & Hands & $\begin{array}{l}\text { Carbon } \\
\text { Adhesive } \\
\text { Tape }\end{array}$ & NR & UPLC-QQQ & $<20 \min$ & $\begin{array}{c}\text { N-NDPA, DPA, MC, } \\
\text { EC }\end{array}$ & $\begin{array}{l}0.01-5 \\
\text { ppm }\end{array}$ \\
\hline Zhao [71] & $\begin{array}{c}7.26 \mathrm{~mm} \\
\begin{array}{c}\text { semiautomatic } 77 \# \\
\text { pistols }\end{array} \\
\end{array}$ & NR & $\begin{array}{l}\text { Hands, } \\
\text { Hair }\end{array}$ & NR & NR & DESI-MS/MS & $<2 \min$ & $\mathrm{EC}, \mathrm{MC}$ & $\begin{array}{c}8-70 \\
\mathrm{pg} / \mathrm{cm}^{3}\end{array}$ \\
\hline Zeichner [72] & $\begin{array}{c}9 \mathrm{~mm} \mathrm{FN} \\
\text { semiautomatic } \\
\text { pistol } \\
\end{array}$ & NR & $\begin{array}{l}\text { Hands, } \\
\text { Hair }\end{array}$ & $\begin{array}{c}\text { Carbon } \\
\text { Adhesive } \\
\text { Tape } \\
\end{array}$ & NR & $\begin{array}{c}\text { IMS } \\
\text { GC-TEA }\end{array}$ & $<20 \min$ & $\begin{array}{c}\text { NG, 2,4-DNT, 2,6- } \\
\text { DNT }\end{array}$ & $\begin{array}{c}0.1-1 \\
\mathrm{ng}\end{array}$ \\
\hline Moran [73] & Glock Model 19 & NR & NR & $\begin{array}{c}\text { PDMS } \\
\text { Membrane }\end{array}$ & NR & GC/MS & NR & $\begin{array}{l}\text { DPA, 2-NDPA, 4- } \\
\text { NDPA, DMP, EC }\end{array}$ & NR \\
\hline Moran [74] & Glock Model 19 & NR & NR & $\begin{array}{c}\text { PDMS } \\
\text { Membrane } \\
\end{array}$ & NR & IMS & $<1$ min & $\begin{array}{l}\text { DPA, N-NDPA, 2- } \\
\text { NDPA, EC } \\
\end{array}$ & NR \\
\hline \multirow{3}{*}{ Zeichner [75] } & \multirow{3}{*}{$\begin{array}{l}\text { 9-mm FN } \\
\text { semiautomatic } \\
\text { pistol }\end{array}$} & \multirow{3}{*}{ NR } & \multirow{3}{*}{ Clothing } & \multirow{3}{*}{$\begin{array}{l}\text { Vacuum } \\
\text { Sampling }\end{array}$} & \multirow{3}{*}{ NR } & IMS & \multirow{3}{*}{$<20 \min$} & \multirow{3}{*}{$\begin{array}{c}\text { NG, 2,4-DNT, 2,6- } \\
\text { DNT, DPA, DNDPA, } \\
\text { N-NDPA, EC }\end{array}$} & $0.3 \mathrm{ng}$ \\
\hline & & & & & & GC-TEA & & & $\begin{array}{c}0.05-1 \\
\mathrm{ng}\end{array}$ \\
\hline & & & & & & GC-MS & & & $\begin{array}{c}\text { Low ng } \\
\text { levels }\end{array}$ \\
\hline Mahoney [76] & NA & NA & $\begin{array}{l}\text { Smokeless } \\
\text { and black }\end{array}$ & $\begin{array}{l}\text { Adhesive } \\
\text { Tape }\end{array}$ & NR & TOF-SIMS & $<10 \min$ & $\mathrm{DBP}, \mathrm{EC}, \mathrm{NC}, \mathrm{NG}$ & NR \\
\hline
\end{tabular}




\begin{tabular}{|c|c|c|c|c|c|c|c|c|c|}
\hline Author & Firearm(s) & $\begin{array}{c}\# \text { of } \\
\text { Shots }\end{array}$ & $\begin{array}{l}\text { Sampling } \\
\text { Area }\end{array}$ & $\begin{array}{l}\text { Collection } \\
\text { Media }\end{array}$ & Storage & Instrumentation & $\begin{array}{l}\text { Analysis } \\
\text { Time }\end{array}$ & $\begin{array}{l}\text { Elements } \\
\text { Detected }\end{array}$ & $\begin{array}{c}\text { Reported Concentration } \\
\text { OR Particle } \\
\text { Ranges/Samples } \\
\end{array}$ \\
\hline $\begin{array}{l}\text { French } \\
{[50]}\end{array}$ & $9 \mathrm{~mm}$ Luger 95 grain & 5 & Hands & $\begin{array}{l}\text { Carbon } \\
\text { Adhesive } \\
\text { Tape }\end{array}$ & $\begin{array}{l}\text { Room } \\
\text { Temp }\end{array}$ & SEM/EDS & NR & NR & 0-691 particles \\
\hline $\begin{array}{l}\text { Lindsay } \\
{[87]}\end{array}$ & $\begin{array}{l}\text { Colt Model New } \\
\text { Frontier Buntline } \\
\text { revolver } \\
\text { Smith \& Wesson } \\
\text { Model } 66 \text { revolver } \\
\text { Glock Model } 22 \\
\text { Para-Ordnance Model } \\
\text { P-12 } 45 \text { pistol }\end{array}$ & $6-10$ & Hands & $\begin{array}{l}\text { Carbon } \\
\text { Adhesive } \\
\text { Tape }\end{array}$ & NR & SEM/EDS & NR & $\mathrm{Pb}, \mathrm{Ba}, \mathrm{Sb}$ & 156-4486 particles \\
\hline $\begin{array}{l}\text { Wrobel } \\
{[70]}\end{array}$ & NR & NR & NR & $\begin{array}{l}\text { Carbon } \\
\text { Adhesive } \\
\text { Tape }\end{array}$ & NR & SEM/EDS & NR & $\begin{array}{l}\mathrm{Al}, \mathrm{Si}, \mathrm{Ca}, \\
\mathrm{S}, \mathrm{K}, \mathrm{Cl}, \mathrm{P}, \\
\mathrm{Na}\end{array}$ & NR \\
\hline $\begin{array}{l}\text { Degaetano } \\
{[72]}\end{array}$ & $\begin{array}{l}\text { Smith and Wesson } \\
\text { Model 10-8 revolver }\end{array}$ & $1-3$ & Hands & $\begin{array}{l}\text { Carbon } \\
\text { Adhesive } \\
\text { Tape }\end{array}$ & $-4 \mathrm{C}$ & SEM/EDS & $60 \mathrm{~min}$ & $\begin{array}{l}\mathrm{Pb}, \mathrm{Ba}, \mathrm{Sb} \\
\mathrm{Zn}, \mathrm{Cu}\end{array}$ & 1-7 particles \\
\hline Toal [7] & $\begin{array}{l}\text { Beretta Px4 Storm } \\
\text { pistol }\end{array}$ & 2 & Hands & $\begin{array}{l}\text { Carbon } \\
\text { Adhesive } \\
\text { Tape XCAT } \\
\text { GSR-600 } \\
\text { sampling } \\
\text { card }\end{array}$ & NR & SEM/EDS & NR & $\begin{array}{l}\mathrm{Ba}, \mathrm{S}, \mathrm{Pb} \\
\mathrm{Sb}, \mathrm{Cu}, \mathrm{Al}, \\
\mathrm{W}, \mathrm{Zn}\end{array}$ & NR \\
\hline $\begin{array}{l}\text { Brozek- } \\
\text { Mucha [51] }\end{array}$ & $\begin{array}{l}\text { Browning } 1906 \text { \& P- } \\
\text { 64; P-83 \& Browning } \\
\text { 1900 \& Beretta } 9 \text { mm } \\
\text { \& Margolin \& TT-33 } \\
\text { pistols }\end{array}$ & 3 & Hands & $\begin{array}{l}\text { Carbon } \\
\text { Adhesive } \\
\text { Tape }\end{array}$ & NR & SEM/EDS & NR & $\begin{array}{l}\mathrm{Pb}, \mathrm{Ba}, \mathrm{Sb}, \\
\mathrm{Sn}\end{array}$ & $100-4000$ particles \\
\hline $\begin{array}{l}\text { Brozek- } \\
\text { Mucha [54] }\end{array}$ & Luger $9 \mathrm{~mm}$ pistol & 1 & Hands & $\begin{array}{l}\text { Carbon } \\
\text { Adhesive } \\
\text { Tape }\end{array}$ & NR & SEM/EDS & $0-4 \mathrm{hrs}$ & $\mathrm{Pb}, \mathrm{Ba}, \mathrm{Sb}$ & $\begin{array}{l}161-11,080 \text { particle range } \\
(3,439 \text { average })\end{array}$ \\
\hline $\begin{array}{l}\text { Zeichner } \\
{[82]}\end{array}$ & $\begin{array}{l}9 \mathrm{~mm} \text { FN semi- } \\
\text { automatic pistol }\end{array}$ & 1 & $\begin{array}{l}\text { Hands \& } \\
\text { Hair }\end{array}$ & $\begin{array}{l}\text { Carbon } \\
\text { Adhesive } \\
\text { Tape }\end{array}$ & NR & SEM/EDS & NR & $\mathrm{Pb}, \mathrm{Ba}, \mathrm{Sb}$ & 21-185 particles \\
\hline
\end{tabular}




\begin{tabular}{|c|c|c|c|c|c|c|c|c|c|}
\hline \multirow{2}{*}{$\begin{array}{l}\text { Coumbaros } \\
{[55]}\end{array}$} & \multirow{2}{*}{$\begin{array}{l}\text { Model 18/3 Smith } \\
\text { and Wesson revolver }\end{array}$} & \multirow{2}{*}{ NR } & \multirow{2}{*}{$\begin{array}{l}\text { Cartridge } \\
\text { case }\end{array}$} & \multirow{2}{*}{ NR } & \multirow{2}{*}{ NR } & \multirow{2}{*}{$\begin{array}{l}\text { TOF-SIMS } \\
\text { SEM/EDS }\end{array}$} & \multirow{2}{*}{$\begin{array}{l}\mathrm{NR} \\
\mathrm{NR}\end{array}$} & \multirow{2}{*}{$\begin{array}{l}\mathrm{Pb}, \mathrm{Ba}, \mathrm{Sb}, \\
\mathrm{Cu} \\
\mathrm{Pb}, \mathrm{Ba}, \mathrm{Sb}, \\
\mathrm{Cu}\end{array}$} & \multirow{2}{*}{$\begin{array}{l}\text { NR } \\
20 \text { particles analyzed }\end{array}$} \\
\hline & & & & & & & & & \\
\hline \multirow[t]{2}{*}{ Chohra [43] } & \multirow[t]{2}{*}{$\begin{array}{l}38 \text { SP Smith \& } \\
\text { Wesson Revolver } \\
\text { Geco }\end{array}$} & \multirow[t]{2}{*}{1} & \multirow{2}{*}{$\begin{array}{l}\text { Hands, } \\
\text { Sleeve, } \\
\text { Shoulder, } \\
\text { Hair }\end{array}$} & \multirow[t]{2}{*}{$\begin{array}{l}\text { Carbon } \\
\text { Adhesive } \\
\text { Tape }\end{array}$} & \multirow[t]{2}{*}{ NR } & NAA & $2 \mathrm{hrs}$ & $\begin{array}{l}\mathrm{Pb}, \mathrm{Ba}, \mathrm{Br}, \\
\mathrm{Sb}, \mathrm{Sn}, \mathrm{Cu}, \\
\mathrm{Ti}, \mathrm{Fe}, \mathrm{Bi}, \\
\mathrm{Zn}, \mathrm{Na}\end{array}$ & NR \\
\hline & & & & & & SEM/EDS & NR & $\begin{array}{l}\mathrm{Pb}, \mathrm{Ba}, \mathrm{Sb}, \\
\mathrm{Si}, \mathrm{Ca}\end{array}$ & 1-645 particles \\
\hline Kage [44] & $\begin{array}{l}0.38 \text {-caliber revolver } \\
\& \text { semiautomatic } \\
\text { pistol }\end{array}$ & $1-19$ & $\begin{array}{l}\text { Hands \& } \\
\text { Clothing }\end{array}$ & $\begin{array}{l}\text { Carbon } \\
\text { Adhesive } \\
\text { Tape }\end{array}$ & NR & SEM/WDS & $3 \mathrm{hrs}$ & $\mathrm{Pb}, \mathrm{Ba}, \mathrm{Sb}$ & NR \\
\hline French [99] & $\begin{array}{l}\text { SIG Sauer P226 9- } \\
\text { mm self-loading } \\
\text { pistol }\end{array}$ & 5 & Hands & $\begin{array}{l}\text { Carbon } \\
\text { Adhesive } \\
\text { Tape }\end{array}$ & NR & SEM/EDS & NR & $\mathrm{Pb}, \mathrm{Ba}, \mathrm{Sb}$ & 14-443 particles \\
\hline $\begin{array}{l}\text { Brozek- } \\
\text { Mucha [134] }\end{array}$ & NR & NR & Hands & $\begin{array}{l}\text { Carbon } \\
\text { Adhesive } \\
\text { Tape }\end{array}$ & NR & SEM/EDS & $4-5 \mathrm{hrs}$ & $\begin{array}{l}\mathrm{Pb}, \mathrm{Ba}, \mathrm{Sb} \\
\mathrm{Sn}\end{array}$ & 1-70 particles \\
\hline Kara [73] & $\begin{array}{l}\text { Sarsilmaz Kilinc } \\
\text { Mega } 2000 \text { brand } \\
\text { pistols }\end{array}$ & 3 & Hands & $\begin{array}{l}\text { Carbon } \\
\text { Adhesive } \\
\text { Tape }\end{array}$ & NR & SEM/EDS & NR & $\mathrm{Pb}, \mathrm{Ba}, \mathrm{Sb}$ & 1603-3911 particles \\
\hline Rijnders [98] & $\begin{array}{l}9 \mathrm{~mm} \text { Sig Sauer } \\
\text { P228 }\end{array}$ & 2 & $\begin{array}{l}\text { Hands, } \\
\text { sleeves, } \\
\text { cloth, } \\
\text { barrel }\end{array}$ & $\begin{array}{l}\text { Cotton Cloth } \\
\text { \& Carbon } \\
\text { Adhesive } \\
\text { Tape }\end{array}$ & NR & SEM/EDS & $10 \min$ & $\begin{array}{l}\mathrm{Pb}, \mathrm{Ba}, \mathrm{Sb} \\
\mathrm{Ti}, \mathrm{Zn}, \mathrm{Gd}\end{array}$ & 5-894 particles \\
\hline $\begin{array}{l}\text { Brozek- } \\
\text { Mucha [106] }\end{array}$ & Škorpion 61E pistol & 6 & $\begin{array}{l}\text { Cloth \& } \\
\text { Skin }\end{array}$ & $\begin{array}{l}\text { Carbon } \\
\text { Adhesive } \\
\text { Tape }\end{array}$ & NR & SEM/EDS & NR & $\begin{array}{l}\mathrm{Pb}, \mathrm{Ba}, \mathrm{Sb}, \\
\mathrm{Cu}\end{array}$ & 50-7000 particles \\
\hline Izzharif [78] & $\begin{array}{l}\text { 0.38 Revolver Smith } \\
\& \text { Wesson \& } 9 \text { mm } \\
\text { semi-automatic } \\
\text { Yavuz } 16 \text { Compact }\end{array}$ & NR & Cloth & $\begin{array}{l}\text { Carbon } \\
\text { Adhesive } \\
\text { Tape }\end{array}$ & NR & SEM/EDS & NR & $\mathrm{Pb}$ & NR \\
\hline
\end{tabular}




\begin{tabular}{|c|c|c|c|c|c|c|c|c|c|}
\hline $\begin{array}{l}\text { Charles } \\
{[77]}\end{array}$ & $\begin{array}{l}9 \mathrm{~mm} \text { model CZ } 75 \\
\text { pistol }\end{array}$ & NR & fabric & $\begin{array}{l}\text { Carbon } \\
\text { Adhesive } \\
\text { Tape }\end{array}$ & NR & SEM/EDS & NR & $\mathrm{Pb}, \mathrm{Ba}, \mathrm{Sb}$ & 3-167 particles \\
\hline $\begin{array}{l}\text { Schwartz } \\
{[75]}\end{array}$ & NR & 1 & Nose & $\begin{array}{l}\text { Blowing Into } \\
\text { Fabric }\end{array}$ & NR & SEM/EDS & NR & $\mathrm{Pb}, \mathrm{Ba}, \mathrm{Sb}$ & 11-542 particles \\
\hline Reyes [76] & $\begin{array}{l}\text { Pistol \& Revolver \& } \\
\text { Shotgun \& } \\
\text { Submachine gun }\end{array}$ & 2 & Nose & $\begin{array}{l}\text { Carbon } \\
\text { Adhesive } \\
\text { Tape }\end{array}$ & NR & SEM/EDS & $6 \mathrm{hrs}$ & $\mathrm{Pb}, \mathrm{Ba}, \mathrm{Sb}$ & 0-1756 particles \\
\hline Kara [80] & $\begin{array}{l}\text { Mega } 2000 \text { Kilinc } \\
\text { model semi-automatic } \\
9 \text { mm Parabellum } \\
\text { pistol \& } 7.65 \mathrm{~mm} \\
\text { Browning pistol }\end{array}$ & NR & Hands & $\begin{array}{l}\text { Carbon } \\
\text { Adhesive } \\
\text { Tape }\end{array}$ & NR & SEM/EDS & NR & $\mathrm{Pb}, \mathrm{Ba}, \mathrm{Sb}$ & 43- 279 particles \\
\hline $\begin{array}{l}\text { Charles } \\
{[110]}\end{array}$ & NR & NR & Hands & $\begin{array}{l}\text { Carbon } \\
\text { Adhesive } \\
\text { Tape }\end{array}$ & NR & SEM/EDS & NR & $\begin{array}{l}\mathrm{Pb}, \mathrm{Ba}, \mathrm{Sb}, \mathrm{Ti}, \\
\mathrm{Zn}\end{array}$ & $0-1550$ particles \\
\hline $\begin{array}{l}\text { Gerard } \\
{[115]}\end{array}$ & $\begin{array}{l}\text { Glock } 19 \text { semi- } \\
\text { automatic pistol }\end{array}$ & $1-2$ & Firearm & $\begin{array}{l}\text { Carbon } \\
\text { Adhesive } \\
\text { Tape }\end{array}$ & NR & SEM/EDS & NR & NR & 0-36 particles \\
\hline $\begin{array}{l}\text { Fojtášek } \\
{[116]}\end{array}$ & CZ 85, 9 mm Luger & 1 & Targets & $\begin{array}{l}\text { Carbon } \\
\text { Adhesive } \\
\text { Tape }\end{array}$ & NR & SEM/EDS & NR & $\mathrm{Pb}, \mathrm{Ba}, \mathrm{Sb}$ & 0-3020 particles \\
\hline $\begin{array}{l}\text { Jalanti } \\
{[118]}\end{array}$ & $\begin{array}{l}\text { Sig Sauer, model } \\
\text { P220, } 9 \text { mm Luger } \\
\text { semi-automatic pistol }\end{array}$ & 1 & Hands & $\begin{array}{l}\text { Carbon } \\
\text { Adhesive } \\
\text { Tape }\end{array}$ & NR & SEM/EDS & NR & $\mathrm{Pb}, \mathrm{Ba}, \mathrm{Sb}$ & $0-187$ particles \\
\hline $\begin{array}{l}\text { Romanò } \\
{[135]}\end{array}$ & Glock Model 17 & 2 & Hands & $\begin{array}{l}\text { Carbon } \\
\text { Adhesive } \\
\text { Tape } \\
\end{array}$ & NR & SEM/EDS & NR & $\begin{array}{l}\mathrm{Pb}, \mathrm{Ba}, \mathrm{Sb}, \mathrm{Zn}, \\
\mathrm{Ti}, \mathrm{Ca}, \mathrm{Cu}, \mathrm{K}, \\
\mathrm{P}, \mathrm{S}, \mathrm{Mg}\end{array}$ & 5-48 particles \\
\hline \multirow{2}{*}{ Merli [71] } & \multirow{2}{*}{$9 \mathrm{~mm}$ Beretta $98 \mathrm{FS}$} & \multirow{2}{*}{ NR } & \multirow{2}{*}{$\begin{array}{l}\text { Entry } \\
\text { points }\end{array}$} & \multirow{2}{*}{$\begin{array}{l}\text { Carbon } \\
\text { Adhesive } \\
\text { Tape Cotton } \\
\text { swabs } \\
\end{array}$} & \multirow{2}{*}{ NR } & ICP-MS & NR & $\mathrm{Ba}, \mathrm{Cu}, \mathrm{Pb}, \mathrm{Sb}$ & $0.19-1.72 \mu \mathrm{g}$ \\
\hline & & & & & & ICP-OES & NR & $\mathrm{Ba}, \mathrm{Cu}, \mathrm{Pb}, \mathrm{Sb}$ & NR \\
\hline
\end{tabular}




\begin{tabular}{|c|c|c|c|c|c|c|c|c|c|}
\hline \multirow{2}{*}{ Merli [71] } & \multirow{2}{*}{$9 \mathrm{~mm}$ Beretta $98 \mathrm{FS}$} & \multirow{2}{*}{ NR } & \multirow{2}{*}{ Entry points } & \multirow{2}{*}{$\begin{array}{l}\text { Carbon } \\
\text { Adhesive Tape } \\
\text { Cotton swabs }\end{array}$} & \multirow{2}{*}{ NR } & ICP-MS & NR & $\mathrm{Ba}, \mathrm{Cu}, \mathrm{Pb}, \mathrm{Sb}$ & $0.19-1.72 \mu \mathrm{g}$ \\
\hline & & & & & & ICP-OES & NR & $\mathrm{Ba}, \mathrm{Cu}, \mathrm{Pb}, \mathrm{Sb}$ & NR \\
\hline Costa $[45]$ & $\begin{array}{l}0.40 \text { caliber pistol \& } \\
0.38 \text { caliber revolver }\end{array}$ & $1-7$ & Hands & $\begin{array}{l}\text { Carbon } \\
\text { Adhesive Tape }\end{array}$ & NR & $\mathrm{ICP} / \mathrm{MS}$ & NR & $\begin{array}{l}\mathrm{Pb}, \mathrm{Ba}, \mathrm{Sb}, \mathrm{Al}, \\
\mathrm{Ti}, \mathrm{Cr}, \mathrm{Mo}, \mathrm{Cu}, \\
\mathrm{Zn}, \mathrm{Sr}\end{array}$ & $\begin{array}{l}0.119-10.9 \mathrm{ng} / \mathrm{mL} \\
(\mathrm{Pb}, \mathrm{Ba}, \mathrm{Sb})\end{array}$ \\
\hline Diaz [33] & $\begin{array}{l}.38, .32, .22, \& 9 \\
\mathrm{~mm}\end{array}$ & $1-62$ & Airborne & $\begin{array}{l}\text { Polycarbonate } \\
\text { Filters }\end{array}$ & NR & $\mathrm{ICP} / \mathrm{MS}$ & NR & $\mathrm{Pb}, \mathrm{Ba}, \mathrm{Sb}$ & $\begin{array}{l}0.002 \mu \mathrm{g} / \mathrm{m}^{3}-58.928 \\
\mu \mathrm{g} / \mathrm{m}^{3}\end{array}$ \\
\hline Lagoo [122] & $\begin{array}{l}9 \text { mm Glock } \\
\text { handgun }\end{array}$ & 11 & Larvae & $\begin{array}{l}\text { Carbon } \\
\text { Adhesive Tape }\end{array}$ & NR & $\mathrm{ICP} / \mathrm{MS}$ & NR & NR & $0.04-2.3 \mu \mathrm{g} / \mathrm{g}$ \\
\hline Halim [93] & $\begin{array}{l}\text { Smith and Wesson } \\
\text { pistol } 0.4 \text {-inch } \\
\text { caliber }\end{array}$ & 1 & Hands & $\begin{array}{l}\text { Moistened } \\
\text { Swabs }\end{array}$ & NR & ICP/OES & NR & $\mathrm{Pb}, \mathrm{Ba}, \mathrm{Cu}$ & $0.098-6.476 \mu \mathrm{g} / \mathrm{mL}$ \\
\hline Merli [81] & Pistol & $1-200$ & Nostrils & Cloth & NR & INAA & NR & $\mathrm{Ba}, \mathrm{Sb}$ & $0.005-50.321 \mu \mathrm{g}$ \\
\hline Gibelli [30] & Franchi revolver & 3 & Pig & Cotton Swabs & NR & NAA & NR & $\mathrm{Sb}$ & $0.07-13.89 \mu \mathrm{g}$ \\
\hline Yuksel [52] & $9 \mathrm{~mm}$ pistol & NR & Hands & $\begin{array}{l}\text { Carbon } \\
\text { Adhesive Tape }\end{array}$ & NR & GFAAS & $30 \mathrm{~min}$ & $\mathrm{~Pb}, \mathrm{Ba}, \mathrm{Sb}$ & 35-800 ng/swab \\
\hline $\begin{array}{l}\text { Schumacher } \\
{[136]}\end{array}$ & DNAG Sintox $9 \mathrm{~mm}$ & 1 & Cloth & $\mathrm{N} / \mathrm{A}$ & NR & $\mathrm{m}-\mathrm{XRF}$ & $2-10 \mathrm{hrs}$ & $\begin{array}{l}\mathrm{Pb}, \mathrm{Sb}, \mathrm{Ba}, \mathrm{Sn}, \\
\mathrm{Zn}, \mathrm{Cu}, \mathrm{Ti}\end{array}$ & NR \\
\hline $\begin{array}{l}\text { Rosenberg } \\
{[32]}\end{array}$ & $\begin{array}{l}.357 \text { caliber Colt } \\
\text { Trooper MK III } \\
\text { revolver }\end{array}$ & 6 & Hands & $\begin{array}{l}\text { Carbon } \\
\text { Adhesive Tape }\end{array}$ & NR & LIBS & NR & $\mathrm{Pb}, \mathrm{Ba}, \mathrm{Sb}$ & NR \\
\hline $\begin{array}{l}\text { Menking- } \\
\text { Hoggatt [41] }\end{array}$ & $\begin{array}{l}\text { Sccy CPX2 TT \& } \\
\text { Taurus } .38 \text { special }\end{array}$ & 5 & Hands & $\begin{array}{l}\text { Carbon } \\
\text { Adhesive Tape }\end{array}$ & $\begin{array}{l}\text { Room } \\
\text { Temp }\end{array}$ & LIBS & $\begin{array}{l}<3 \\
\text { minutes }\end{array}$ & $\begin{array}{l}\mathrm{Pb}, \mathrm{Ba}, \mathrm{Sb}, \mathrm{Cu}, \\
\mathrm{Al}\end{array}$ & NR \\
\hline \multirow{2}{*}{$\begin{array}{l}\text { Dona- } \\
\text { Fernández } \\
{[34]}\end{array}$} & \multirow{2}{*}{ NR } & \multirow{2}{*}{ NR } & \multirow{2}{*}{ Hands } & \multirow{2}{*}{$\begin{array}{l}\text { Carbon } \\
\text { Adhesive Tape }\end{array}$} & \multirow{2}{*}{ NR } & Portable LIBS & NR & $\mathrm{Pb}, \mathrm{Ba}, \mathrm{Sb}$ & NR \\
\hline & & & & & & SEM/EDS & NR & $\mathrm{Pb}, \mathrm{Ba}, \mathrm{Sb}$ & $1-100$ \\
\hline
\end{tabular}




\begin{tabular}{|c|c|c|c|c|c|c|c|c|c|}
\hline \multirow{2}{*}{$\begin{array}{l}\text { Fambro } \\
{[35]}\end{array}$} & \multirow{2}{*}{ N/A } & \multirow{2}{*}{ N/A } & \multirow{2}{*}{$\begin{array}{l}\text { Bomb } \\
\text { Calorimeter }\end{array}$} & \multirow{2}{*}{$\begin{array}{l}\text { Collection } \\
\text { Cards }\end{array}$} & \multirow{2}{*}{ NR } & LIBS & NR & $\begin{array}{l}\mathrm{Ca}, \mathrm{Na}, \mathrm{Sr}, \mathrm{K}, \\
\mathrm{Ba}, \mathrm{S}, \mathrm{Fe}\end{array}$ & NR \\
\hline & & & & & & SEM/EDS & NR & $\mathrm{K}, \mathrm{Cl}, \mathrm{Ba}, \mathrm{S}, \mathrm{Sr}$ & NR \\
\hline \multirow{2}{*}{$\begin{array}{l}\text { Fambro } \\
{[36]}\end{array}$} & \multirow{2}{*}{ Walther CCP 9 mm } & \multirow{2}{*}{1 or 5} & \multirow{2}{*}{ Hands } & \multirow{2}{*}{$\begin{array}{l}\text { Carbon } \\
\text { Adhesive } \\
\text { Tape }\end{array}$} & \multirow{2}{*}{ NR } & LIBS & NR & $\mathrm{Ba}, \mathrm{Ca}, \mathrm{Na}, \mathrm{K}$ & NR \\
\hline & & & & & & SEM/EDS & NR & $\mathrm{Al}, \mathrm{Si}, \mathrm{K}, \mathrm{Ba}$ & NR \\
\hline \multirow[b]{2}{*}{$\begin{array}{l}\text { Szynkowsk } \\
\text { am [137] }\end{array}$} & \multirow[b]{2}{*}{ FAM $12 \mathrm{~mm}$ shotgun } & \multirow[b]{2}{*}{ N/A } & \multirow{2}{*}{$\begin{array}{l}\text { Steel, } \\
\text { laminated } \\
\text { wood, glass, } \\
\text { cell phone }\end{array}$} & \multirow{2}{*}{$\begin{array}{l}\text { Scotch } \\
\text { Tape, } \\
\text { Remco } \\
\text { Adhesive, } \\
\text { Filmolux } \\
\text { Adhesive }\end{array}$} & \multirow[b]{2}{*}{ NR } & TOF-SIMS & NR & $\mathrm{Na}, \mathrm{K}, \mathrm{Ba}, \mathrm{Pb}$ & NR \\
\hline & & & & & & SEM/EDS & NR & $\begin{array}{l}\mathrm{Al}, \mathrm{Si}, \mathrm{Ca}, \mathrm{Ba}, \\
\mathrm{Pb}\end{array}$ & NR \\
\hline \multirow{2}{*}{$\begin{array}{l}\text { Seyfang } \\
{[26]}\end{array}$} & \multirow[t]{2}{*}{ 10/22 Ruger pistol } & \multirow[t]{2}{*}{1} & \multirow[t]{2}{*}{ Hands } & \multirow{2}{*}{$\begin{array}{l}\text { Carbon } \\
\text { Adhesive } \\
\text { Tape }\end{array}$} & \multirow[t]{2}{*}{ NR } & TOF-SIMS & $3 \mathrm{~min}$ & $\begin{array}{l}\mathrm{Na}, \mathrm{Mg}, \mathrm{Al}, \mathrm{Si} \\
\mathrm{K}, \mathrm{Ca}, \mathrm{Ti}, \mathrm{Mn}, \\
\mathrm{Fe}, \mathrm{Cu}, \mathrm{Li}, \mathrm{Sr}, \\
\mathrm{Zr}, \mathrm{Pb}, \mathrm{Sb},\end{array}$ & NR \\
\hline & & & & & & SEM/EDS & NR & $\begin{array}{l}\mathrm{K}, \mathrm{Ca}, \mathrm{Ba}, \mathrm{Cu}, \\
\mathrm{Na}, \mathrm{Ga}, \mathrm{Mg}, \mathrm{Pt}, \\
\mathrm{Pb}, \mathrm{Si}, \mathrm{Al}, \mathrm{Sn}\end{array}$ & NR \\
\hline
\end{tabular}




\begin{tabular}{|c|c|c|c|c|c|c|c|c|c|}
\hline Author & Firearm & $\begin{array}{l}\# \quad \text { of } \\
\text { Shots }\end{array}$ & $\begin{array}{l}\text { Sampling } \\
\text { Area }\end{array}$ & $\begin{array}{l}\text { Collection } \\
\text { Media }\end{array}$ & Storage & Instrumentation & $\begin{array}{l}\text { Analysis } \\
\text { Time }\end{array}$ & Compounds Detected & $\begin{array}{l}\text { Concentration/ } \\
\text { Particle Counts }\end{array}$ \\
\hline \multirow{2}{*}{$\begin{array}{l}\text { Trejos } \\
{[11]}\end{array}$} & $\begin{array}{l}9 \mathrm{~mm} \text { Luger } \\
0.38 \text { Special }\end{array}$ & \multirow{2}{*}{5} & \multirow{2}{*}{ Hands } & \multirow{2}{*}{$\begin{array}{l}\text { Carbon } \\
\text { Adhesive } \\
\text { Tape }\end{array}$} & \multirow{2}{*}{$\begin{array}{l}\text { Room } \\
\text { Temp. }\end{array}$} & LIBS & $<1 \min$ & $\begin{array}{l}\mathrm{Pb}, \mathrm{Ba}, \mathrm{Sb}, \mathrm{Cu}, \mathrm{Al}, \mathrm{Ca}, \mathrm{Sr}, \mathrm{Ti}, \\
\mathrm{Zn},\end{array}$ & $\begin{array}{l}0.1 \quad-440 \mathrm{ng} \\
(\mathrm{MDL})\end{array}$ \\
\hline & $\begin{array}{l}0.22 \text { Long Rifle } \\
9 \mathrm{~mm} \text { Luger }\end{array}$ & & & & & Electrochemical & $<3 \min$ & $\mathrm{Pb}, \mathrm{Sb}, \mathrm{NG}, 2,4-\mathrm{DNT}$ & $\begin{array}{l}0.1-1.0 \mathrm{mg} / \mu \mathrm{L} \\
(\mathrm{MDL})\end{array}$ \\
\hline Bell [12] & $\begin{array}{l}\text { Glock } 179 \mathrm{~mm} \\
\text { semiauto pistol } \quad \mathrm{S} \& \mathrm{~W} \\
0.38 \text { revolver. }\end{array}$ & $1-3$ & Hands & $\begin{array}{l}\text { Polymer } \\
\text { Tesa Tack }\end{array}$ & $\begin{array}{l}\text { Stored at } \\
4^{\circ} \mathrm{C} \text { freezer }\end{array}$ & LC/QQQ & $<15 \min$ & $\begin{array}{l}\text { DPA, EC, MC, NNDPA, 4- } \\
\text { NDPA } \\
\mathrm{Ba}, \mathrm{Pb}, \mathrm{Fe}, \mathrm{Ca}\end{array}$ & $\begin{array}{l}0.02-12 \mathrm{ng} \\
(\mathrm{MDL})\end{array}$ \\
\hline \multirow[t]{2}{*}{ Gandy [5] } & \multirow[t]{2}{*}{ NR } & \multirow[t]{2}{*}{ NR } & \multirow[t]{2}{*}{ NR } & \multirow[t]{2}{*}{ NR } & \multirow[t]{2}{*}{ NR } & Color tests & NR & $\begin{array}{l}\text { DPA, MC, EC Resorcinol, } \\
\text { 2,4-DNT, 2,6-DNT, 3,4- } \\
\text { DNT,2,4,6- TNT, carbazole }\end{array}$ & $0.1-5$ ug (MDL) \\
\hline & & & & & & SEM/EDS & NR & $\begin{array}{l}\mathrm{Pb}, \mathrm{PbNO}_{3}, \mathrm{Ba}, \mathrm{BaNO}_{3}, \mathrm{Sb} \\
\mathrm{SbS}, \mathrm{Zn}, \mathrm{Ti}, \mathrm{Cu}, \mathrm{Fe}\end{array}$ & NR \\
\hline \multirow{2}{*}{ Ali [13] } & \multirow{2}{*}{$\begin{array}{lr}38 \text { special revolver with } \\
\text { LRN Blazer } 158 \text { grade } \\
\text { ammunition } & 45 \\
\text { automatic with FMJ- } \\
\text { Independence } 230 \text { grade } \\
\text { ammunition }\end{array}$} & \multirow{2}{*}{ NR } & \multirow{2}{*}{ Hands } & \multirow{2}{*}{$\begin{array}{l}\text { Carbon } \\
\text { Adhesive } \\
\text { Tape }\end{array}$} & \multirow{2}{*}{ room temp } & LC/QQQ & \multirow{2}{*}{$14 \min$} & $\begin{array}{l}\text { AKII, EC, N, N-DPA, } \\
\text { NDPA, }\end{array}$ & $\begin{array}{l}1.16 \times 10^{-4}- \\
4.65 \times 10^{-4} \mu \mathrm{g} / \mathrm{mL} \\
(\mathrm{MDL})\end{array}$ \\
\hline & & & & & & SEM/EDS & & $\mathrm{Pb}, \mathrm{Ba}, \mathrm{Sb}, \mathrm{Zn}$ & 1- 856 particles \\
\hline \multirow{2}{*}{ Abrego [14] } & \multirow{2}{*}{$\begin{array}{l}\text { Fiocchi } 9 \mathrm{~mm} \text { Luger ZP } \\
9 \mathrm{~mm} \text { Heckler \& Koch } \\
\text { pistol model USP } \\
\text { Compact } \\
\text { Sellier \& Bellot } 9 \mathrm{~mm} \\
\text { Luger FMJ } \\
\end{array}$} & \multirow{2}{*}{$1-6$} & \multirow{2}{*}{ Hands } & \multirow{2}{*}{$\begin{array}{l}\text { Carbon } \\
\text { Adhesive } \\
\text { Tape }\end{array}$} & \multirow[t]{2}{*}{ NR } & $\begin{array}{l}\text { micro-Raman } \\
\text { spectroscopy }\end{array}$ & \multirow{2}{*}{$1 \mathrm{hr}$} & $\begin{array}{l}\text { MC, EC, DPA, 2-NDPA, 4- } \\
\text { NDPA, 2,4-DNDPA }\end{array}$ & NR \\
\hline & & & & & & SLA-ICPMS & & $\mathrm{Pb}, \mathrm{Ba}, \mathrm{Ti}, \mathrm{Sn}, \mathrm{Sr}, \mathrm{Ni}, \mathrm{Cu}, \mathrm{Zr}$ & 1381 particles \\
\hline \multirow{3}{*}{ Tarifa [15] } & \multirow{3}{*}{$\begin{array}{l}\text { American Eagle (Federal } \\
\text { Cartridge Company) } 9 \\
\text { mm Luger, } 124 \text { GR. full } \\
\text { metal jacket }\end{array}$} & \multirow{3}{*}{$\begin{array}{l}128 \\
\text { total }\end{array}$} & \multirow{3}{*}{ Hands } & \multirow{3}{*}{$\begin{array}{l}\text { Carbon } \\
\text { Adhesive } \\
\text { Tape }\end{array}$} & \multirow{3}{*}{ NR } & CMV GC-MS & $<12 \min$ & NG, 2,4-DNT, DPA & $\begin{array}{l}\begin{array}{l}3.1-8.2 \\
(\mathrm{MDL})\end{array} \\
\end{array}$ \\
\hline & & & & & & LIBS & NR & $\begin{array}{l}\mathrm{Pb}, \mathrm{Ba}, \mathrm{Sb}, \mathrm{Al}, \mathrm{Ca}, \mathrm{Cu}, \mathrm{Cr}, \mathrm{Fe}, \\
\mathrm{K}, \mathrm{Li}, \mathrm{Mg}, \mathrm{Mn}, \mathrm{Ni}, \mathrm{P}, \mathrm{S}, \mathrm{Si}, \mathrm{Sn}, \\
\mathrm{Sr}, \mathrm{Ti}, \mathrm{Zn}\end{array}$ & $\begin{array}{l}65-782 \mathrm{ng} \\
(\mathrm{MDL})\end{array}$ \\
\hline & & & & & & ICP-OES & NR & $\begin{array}{l}\mathrm{Pb}, \mathrm{Ba}, \mathrm{Sb}, \mathrm{Al}, \mathrm{Ca}, \mathrm{Cu}, \mathrm{Cr}, \mathrm{Fe}, \\
\mathrm{K}, \mathrm{Li}, \mathrm{Mg}, \mathrm{Mn}, \mathrm{Ni}, \mathrm{P}, \mathrm{S}, \mathrm{Si}, \mathrm{Sn} \\
\mathrm{Sr}, \mathrm{Ti}, \mathrm{Zn}\end{array}$ & $\begin{array}{l}21-9767 \text { ng } \\
(\mathrm{MDL})\end{array}$ \\
\hline \multirow[b]{2}{*}{ Morelato [16] } & \multirow{2}{*}{$\begin{array}{l}\text { Glock Model } 17 \text { and } 21 \\
\text { Desert Eagle Pistol \& } \\
\text { Smith \& Wesson Model } \\
65 / 1 \text { and } 17 / 8\end{array}$} & \multirow[b]{2}{*}{3} & \multirow[b]{2}{*}{ Hands } & \multirow{2}{*}{$\begin{array}{l}\text { Carbon } \\
\text { Adhesive } \\
\text { Tape }\end{array}$} & \multirow{2}{*}{$4^{\circ} \mathrm{C}$ freezer } & DESI-MS/MS & NR & EC, DPA, nitrated-DPAs & NR \\
\hline & & & & & & SEM/EDS & NR & $\mathrm{Pb}, \mathrm{Ba}, \mathrm{Sb}$ & 5-10 analyzed \\
\hline
\end{tabular}




\subsection{Conclusions and Future Considerations}

Understanding the variables affecting OGSR and IGSR after discharging a firearm, plays a crucial role in the interpretation of the evidence. Considering the differing ways GSR distributes, transfers, and persists aids in the sampling and analysis of evidence. However, challenges associated with this include accurate sampling and collection of GSR, performing rapid yet reliable analytical methods, and providing ample information about a crime scene sample for appropriate interpretation of results.

While there are several types of GSR sampling media, the most commonly chosen in research and casework is the carbon adhesive tape attached to a standard SEM stub, due to its versatility and established use by crime scene personnel. As proven above, OGSR compounds and IGSR particles can transfer and persist on areas of the body other than the hands. Carbon adhesive stubs eliminate the need for multiple collection tools, as they are applied to not only hands, but other surfaces including a person's face, hair, clothing, and most recently, their nostrils. Future infield collection should focus on other sampling areas where GSR particles tend to persist longer, such as the forehead of a person's face, the hair located on the top of their head, and roughly textured clothing.

After understanding the behavior of GSR for proper collection, integration of multiple instrumental methods becomes crucial. Advances in GSR analysis and complexity of interpretation, leads to the use of rapid combined strategies for the detection of both organic and inorganic components. This is illustrated by the incorporation of organic analyses, such as LC/MS or DESI/MS, in conjunction with confirmatory SEM/EDS analysis. Additionally, exploration of novel combinations such as LIBS with electrochemistry, CMV-GC/MS with LIBS, FTIR with Raman, and LC-MS/MS with the use of complexing agents, have shown great promise for 
identifying both types of GSR markers from a single sample. The orthogonal analysis is vital for the evolving market of non-toxic ammunition, which is replacing typical IGSR heavy metals with other materials, such as organic compounds.

Although SEM remains the standard for confirmatory GSR analysis, several studies have been conducted suggesting the use of other instrumentation methods. Instruments such as LIBS, GC/MS, LC/MS, and Raman are already standard in crime labs for multiple sample types and provide additional screening and confirmatory options to compliment SEM/EDS. While these methods are not able to perform single particle analysis, identification of important OGSR and IGSR markers is still possible down to ppm and ppt levels. Further research would allow for increased incorporation of faster instrumentation techniques in casework. The improvement of GSR detection, analysis, and interpretation lies in the combination of rapid, simultaneous, and complimentary IGSR and OGSR analytical methods on a single sample to increase understanding, confidence, and the value of results.

The interest of the forensic community to increase the body of knowledge in this area and produce complementary consensus-based methods is reflected by current efforts of expert GSR groups such as the Organization of Scientific Area Committees (NIST-OSAC), the National Institute of Justice's Forensic Science Research and Development Technology Working Group (TWG) and the European Network of Forensic Science Institute (ENFSI). We anticipate this review will serve as a valuable reference to the forensic practitioners and researchers in this field, particularly in the areas of collection, persistence, and analysis of IGSR and OGSR of modern ammunition. 


\subsection{References for Chapter 3}

[1] L.S. Blakey, G.P. Sharples, K. Chana, J.W. Birkett, Fate and behavior of gunshot residue-A review, J. Forensic Sci. 63 (2018) 9-19, https://doi.org/10.1111/1556-4029.13555. [2] ASTM, Standard Practice for Gunshot Residue Analysis by Scanning Electron Microscopy/Energy Dispersive X-Ray Spectrometry, B. Stand. (2016) 1-5. https://doi.org/10.1520/E1588-17.

[3] M. Roberts, N. Petraco, M. Gittings, Novel method for the detection of nitroglycerin in smokeless powders, Sci. Justice. 55 (2015) 467-471, https://doi.org/10.1016/j.scijus.2015.08.001.

[4] M. López-López, C. García-Ruiz, Recent non-chemical approaches to estimate the shooting distance, Forensic Sci. Int. 239 (2014) 79-85, https://doi.org/10.1016/j.forsciint.2014.03.023.

[5] L. Gandy, K. Najjar, M. Terry, C. Bridge, A novel protocol for the combined detection of organic, inorganic gunshot residue, Forensic Chem. 8 (2018) 1-10, https://doi.org/10.1016/j.forc.2017.12.009.

[6] B. Yeager, K. Bustin, J. Stewart, R. Dross, S. Bell, Evaluation and validation of ion mobility spectrometry for presumptive testing targeting the organic constituents of firearms discharge residue, Anal. Methods. 7 (2015) 9683-9691, https://doi.org/10.1039/c5ay02417j.

[7] S. Toal, W. Niemeyer, S. Conte, D. Montgomery, G. Ericksen, Spie, Confirmatory analysis of field-presumptive GSR test sample using SEM/EDS, in: Conf. Scanning Microsc., Spie-Int Soc Optical Engineering, BELLINGHAM (2014), https://doi.org/10.1117/12.2074212. [8] O. Dalby, D. Butler, J. Birkett, Analysis of gunshot residue and associated materials - A review, J. Forensic Sci. 55 (2010) 924-943, https://doi.org/10.1111/j.1556-4029.2010.01370.x. 
[9] E. Goudsmits, G. Sharples, J. Birkett, Recent trends in organic gunshot residue analysis, TrAC - Trends Anal. Chem. 74 (2015) 46-57, https://doi.org/10.1016/j.trac.2015.05.010.

[10] K. Chang, P. Jayaprakash, C. Yew, A. Abdullah, Gunshot residue analysis and its evidential values: A review, Aust. J. Forensic Sci. 45 (2013) 3-23, https://doi.org/10.1080/00450618.2012.691546.

[11] T. Trejos, C. Vander Pyl, K. Menking-Hoggatt, A. Alvarado, L. Arroyo, Fast identification of inorganic and organic gunshot residues by LIBS and electrochemical methods, Forensic Chem. 8 (2018) 146-156, https://doi.org/10.1016/j.forc.2018.02.006.

[12] S. Bell, W. Feeney, Single shot, single sample, single instrument detection of IGSR and OGSR using LC/MS/MS, Forensic Sci. Int. 299 (2019) 215-222, https://doi.org/10.1016/j.forsciint.2019.04.002.

[13] Z. Abrego, N. Grijalba, N. Unceta, M. Maguregui, A. Sanchez, A. Fernandez-Isla, M. Goicolea, R. Barrio, A novel method for the identification of inorganic and organic gunshot residue particles of lead-free ammunitions from the hands of shooters using scanning laser ablation-ICPMS and Raman micro-spectroscopy, Analyst 139 (2014) 6232-6241, https://doi.org/10.1039/c4an01051e.

[14] A. Tarifa, J.R. Almirall, Fast detection and characterization of organic and in- organic gunshot residues on the hands of suspects by CMV-GC-MS and LIBS, Sci. Justice. 55 (2015) 168-175, https://doi.org/10.1016/j.scijus.2015.02.003.

[15] M. Morelato, A. Beavis, A. Ogle, P. Doble, P. Kirkbride, C. Roux, Screening of gunshot residues using desorption electrospray ionisation-mass spectrometry (DESI-MS), Forensic Sci. Int. 217 (2012) 101-106, https://doi.org/10.1016/j.forsciint.2011.10.030. 
[16] L. Ali, K. Brown, H. Castellano, S. Wetzel, A Study of the presence of gunshot residue in Pittsburgh police stations using SEM/EDS and LC-MS/MS, J. Forensic Sci. 61 (2016) 928-938, https://doi.org/10.1111/1556-4029.13077.

[17] Y. Cao, R. Linnen, D. Good, I. Samson, R. Epstein, The application of portable XRF and Benchtop SEM-EDS to Cu-Pd exploration in the Coldwell alkaline complex, Ontario, Canada, Geochem. Explor. Environ. Anal. 16 (2016) 193-212, https:// doi.org/10.1144/geochem2015394.

[18] K. Mason, R. Wuhrer, Phenom Desktop SEM for Gunshot Residue and Cathodoluminescence Imaging and Analysis, Microsc. Microanal. 23 (2017) 1074-1075, https://doi.org/10.1017/s1431927617006031.

[19] K. Mason, A forensic mineralogy toolbox - The next generation of instrumentation for forensic applications, in: Int. Microsc. Congr. (IMC Prague 2014), 2014: pp. 1-2.

[20] R. Berk, Automated SEM/EDS analysis of airbag residue I: Particle identification, J. Forensic Sci. 54 (2009) 60-68, https://doi.org/10.1111/j.1556-4029.2008.00918.x.

[21] R. Berk Automated, SEM/EDS analysis of airbag residue. II: Airbag residue as a source of percussion primer residue particles, J. Forensic Sci. 54 (2009) 69-76, https://doi.org/10.1111/j.1556-4029.2008.00919.x.

[22] R. Popelka-Filcoff, K. Kirkbride, K. Seyfang, N. Lucas, W. Tucker, Gunshot residue and brake pads: Compositional and morphological considerations for forensic casework, Forensic Sci. Int. 270 (2016) 76-82, https://doi.org/10.1016/j.

[23] G. Ingo, M. D’Uffizi, G. Falso, G. Bultrini, G. Padeletti, Thermal and microchemical investigation of automotive brake pad wear residues, Thermochim. Acta. 418 (2004) 61-68, https://doi.org/10.1016/j.tca.2003.11.042. 
[24] P. Mosher, M. McVicar, E. Randall, E. Sild, Gunshot residue-similar particles produced by fireworks, J. Can. Soc. Forensic Sci. 31 (1998) 157-168, https://doi.org/10.1080/00085030.1998.10757115.

[25] Z. Brozek-Mucha, Chemical and physical characterisation of welding fume particles for distinguishing from gunshot residue, Forensic Sci. Int. 254 (2015) 51-58, https://doi.org/10.1016/j.forsciint.2015.06.033.

[26] K. Seyfang, N. Lucas, K. Redman, R. Popelka-Filcoff, H. Kobus, K. Kirkbride, Glasscontaining gunshot residues and particles of industrial and occupational origins: Considerations for evaluating GSR traces, Forensic Sci. Int. 298 (2019) 284-297, https://doi.org/10.1016/j.forsciint.2019.03.010.

[27] K. Seyfang, N. Lucas, R. Popelka-Filcoff, H. Kobus, K. Redman, K. Kirkbride, Methods for analysis of glass in glass-containing gunshot residue (gGSR) particles, Forensic Sci. Int. 298 (2019) 359-371, https://doi.org/10.1016/j.forsciint.2019.03.019.

[28] M. Trimpe, Analysis of fireworks for particles of the type found in primer residue (GSR), Int. Assoc. Microanal. 4 (2003) 1-7.

[29] M. Donghi, K. Mason, F.S. Romolo, Detecting Gunshot Residue from Sellier \& Bellot Nontox Heavy Metal-free Primer by in situ Cathodoluminescence, J. Forensic Sci. 64 (2019) 1658-1667, https://doi.org/10.1111/1556-4029.14110.

[30] D. Gibelli, A. Brandone, S. Andreola, D. Porta, E. Giudici, M. Grandi, C. Cattaneo, Macroscopic, microscopic, and chemical assessment of gunshot lesions on de- composed pig skin, J. Forensic Sci. 55 (2010) 1092-1097, https://doi.org/10.1111/j.1556-4029.2010.01378.x. [31] S. Goode, C. Dockery, M. Bachmeyer, A. Nieuwland, S. Morgan, Detecting gunshot residue by laser induced breakdown spectroscopy, Laser Induc, Plasma Spectrosc. App. (2002). 
[32] M. Rosenberg, C. Dockery, Determining the lifetime of detectable amounts of gunshot residue on the hands of a shooter using laser-induced breakdown spectroscopy, Appl. Spectrosc. 62 (2008) 1238-1241, https://doi.org/10.1366/000370208786401473.

[33] E. Diaz, J. Souza Sarkis, S. Viebig, P. Saldiva, Measurement of airborne gunshot particles in a ballistics laboratory by sector field inductively coupled plasma mass spectrometry, Forensic Sci. Int. 214 (2012) 44-47, https://doi.org/10.1016/j.forsciint.2011.07.016.

[34] A. Doña-Fernández, I. de Andres-Gimeno, P. Santiago-Toribio, E. Valtuille-Fernández, F. Aller-Sanchez, A. Heras-González, Real-time detection of GSR particles from crime scene: A comparative study of SEM/EDX and portable LIBS system, Forensic Sci. Int. 292 (2018) 167175, https://doi.org/10.1016/j.forsciint.2018.09.021.

[35] L. Fambro, E. Miller, D. Vandenbos, C. Dockery, Characterization of lead-free gunshot residue analogs, Anal. Methods. 8 (2016) 3132-3139, https://doi.org/10.1039/c6ay00725b.

[36] L. Fambro, D. Vandenbos, M. Rosenberg, C. Dockery, Laser-induced breakdown spectroscopy for the Rapid characterization of lead-free gunshot residues, Appl. Spectrosc. 71 (2017) 699-708, https://doi.org/10.1177/0003702816689099.

[37] M. Sampedro, M. Aliste, S. Arranz, S. Alicia, N. Unceta, G. Alberto, A. Vallejo, Particle analysis for the detection of gunshot residue (GSR) in nasal samples using scanning Laser Ablation and Inductively Coupled Plasma-Mass Spectrometry, J. Forensic Sci. (2020), https://doi.org/10.1111/1556-4029.14278.

[38] D. Hahn, N. Omenetto, Laser-Induced Breakdown Spectroscopy (LIBS), part II: Review of instrumental and methodological approaches to material analysis and applications to different fields, Appl. Spectrosc. 66 (2012) 347-419, https://doi.org/10.1366/11-06574. 
[39] M. Silva, J. Cortez, C. Pasquini, R. Honorato, A. Paim, M. Pimentel, Gunshot residues:

Screening analysis by laser-induced breakdown spectroscopy, J. Braz. Chem. Soc. 20 (2009) 1887-1894, https://doi.org/10.1590/S0103-50532009001000017.

[40] C. Dockery, S. Goode, Laser-induced breakdown spectroscopy for the detection of gunshot residues on the hands of a shooter, Appl. Opt. 42 (2007) 6153, https://doi.org/10.1364/ao.42.006153.

[41] K. Menking-Hoggatt, L. Arroyo, J. Curran, T. Trejos, Novel LIBS method for microspatial chemical analysis of inorganic gunshot residues, J. Chemom. (2019) 1-13, https://doi.org/10.1002/cem.3208.

[42] J. Arndt, S. Bell, L. Crookshanks, M. Lovejoy, C. Oleska, T. Tulley, D. Wolfe, Preliminary evaluation of the persistence of organic gunshot residue, Forensic Sci. Int. (2012) 137-145, https://doi.org/10.1016/j.forsciint.2012.05.011.

[43] J. Moran, S. Bell, Skin permeation of organic gunshot residue: Implications for sampling and analysis, Anal. Chem. 86 (2014) 6071-6079, https://doi.org/10.1021/ac501227e.

[44] B. Stevens, S. Bell, K. Adams, Initial evaluation of inlet thermal desorption GC-MS analysis for organic gunshot residue collected from the hands of known shooters, Forensic Chem. 2 (2016) 55-62, https://doi.org/10.1016/j.forc.2016.10.001.

[45] M. Lopez-Lopez, M. De La Ossa, C. Garcia-Ruiz, Fast analysis of complete macroscopic gunshot residues on substrates using Raman imaging, Appl. Spectrosc. 69 (2015) 889-893, https://doi.org/10.1366/14-07816.

[46] M. López-López, J.J. Delgado, C. García-Ruiz, Ammunition identification by means of the organic analysis of gunshot residues using Raman spectroscopy, Anal. Chem. 84 (2012) 3581-3585, https://doi.org/10.1021/ac203237w. 
[47] M. Maitre, K. Kirkbride, M. Horder, C. Roux, A. Beavis, Thinking beyond the lab:

Organic gunshot residues in an investigative perspective, Aust. J. Forensic Sci. 50 (2018) 659665, https://doi.org/10.1080/00450618.2018.1457718.

[48] M. Zhao, S. Zhang, C. Yang, Y. Xu, Y. Wen, L. Sun, X. Zhang, Desorption electrospray tandem MS (DESI-MSMS) analysis of methyl centralite and ethyl centralite as gunshot residues on skin and other surfaces, J. Forensic Sci. 53 (2008) 807-811, https://doi.org/10.1111/j.1556-4029.2008.00752.x.

[49] A. Zeichner, B. Eldar, A novel method for extraction and analysis of gunpowder residues on double-side adhesive coated stubs, J. Forensic Sci. 49 (2004) 1-13, https://doi.org/10.1520/jfs2004022.

[50] J. Bueno, I. Lednev, Attenuated total reflectance-FT-IR imaging for rapid and automated detection of gunshot residue, Anal. Chem. 86 (2014) 3389-3396, https://doi.org/10.1021/ac4036718.

[51] J. Bueno, L. Halamkova, A. Rzhevskii, I.K. Lednev, Raman microspectroscopic mapping as a tool for detection of gunshot residue on adhesive tape, Anal. Bioanal. Chem. 410 (2018) 7295-7303, https://doi.org/10.1007/s00216-018-1359-1.

[52] J. Moran, S. Bell, Analysis of organic gunshot residue permeation through a model skin membrane using ion mobility spectrometry, Int. J. Ion Mobil. Spectrom. 16 (2013) 247-258, https://doi.org/10.1007/s12127-013-0138-0.

[53] A. Zeichner, B. Eldar, B. Glattstein, A. Koffman, T. Tamiri, D. Muller, Vacuum collection of gunpowder residues from clothing worn by shooting suspects, and their analysis by GC/TEA, IMS, and GC/MS, J. Forensic Sci. 48 (2003) 2002390, https://doi.org/10.1520/jfs2002390. 
[54] C. Hofstetter, M. Maitre, A. Beavis, C. Roux, C. Weyermann, A. Gassner, A study of transfer and prevalence of organic gunshot residues, Forensic Sci. Int. 277 (2017) 241-251, https://doi.org/10.1016/j.forsciint.2017.06.013.

[55] Z. Brozek-Mucha, Z. Brożek-Mucha, Trends in analysis of gunshot residue for forensic purposes, Anal. Bioanal. Chem. 409 (2017) 5803-5811, https://doi.org/10.1007/s00216-017$\underline{0460-1 .}$

[56] M. Houck, S. Charles, B. Nys, N. Guensens, 18th INTERPOL International Forensic Science Managers Symposium, (2016) 69-89.

[57] M. Houck, S. Charles, N. Guensens, E. Vergalito, B. Nys, 19th INTERPOL International Forensic Science Managers Symposium, (2019) 39-67.

[58] L. Filho, US6786986B2 Non-toxic composition for priming mixture for small ca- liber arms ammunition, 2004. https://doi.org/10.1038/incomms 1464 .

[59] I. Weber, M. Albino de Carvalho, C. Pasquini, S. Junior, J. Amigo, M. Pimentel, M. Talhavini, NIR hyperspectral images for identification of gunshot residue from tagged ammunition, Anal. Methods. 10 (2018) 4711-4717, https://doi.org/10.1039/c8ay01341a. [60] F. Romolo, M. Bailey, J. de Jesus, L. Manna, M. Donghi, Unusual sources of Sn in GSR. An experimental study by, SEM and IBA, Sci. Justice. 59 (2019) 181-189, https://doi.org/10.1016/J.SCIJUS.2018.10.009.

[61] H. Meng, B. Caddy, Gunshot residue analysis: A Review, J Forensic Sci. 42 (1997) 553570.

[62] M. Maitre, K. Kirkbride, M. Horder, C. Roux, A. Beavis, Current perspectives in the interpretation of gunshot residues in forensic science: A review, Forensic Sci. Int. 270 (2017) 111, https://doi.org/10.1016/j.forsciint.2016.09.003. 
[63] R. Taudte, A. Beavis, L. Blanes, N. Cole, P. Doble, C. Roux, Detection of gunshot residues using mass spectrometry, Biomed Res. Int. 2014 (2014) 1-16, https://doi.org/10.1155/2014/965403.

[64] J. Bailey, Analysis of bullet wipe patterns on cloth targets, J. Forensic Identif. 55 (2005) $448-460$.

[65] H. Wrobel, J. Millar, M. Kijek, M. Millar, M. Kijek, Comparison of properties of adhesive tapes, tabs, and liquids used for the collection of gunshot residue and other trace materials for SEM analysis, J. Forensic Sci. 43 (1998) 178-181, https://doi.org/10.1520/jfs16107j.

[66] D. Merli, A. Amadasi, D. Mazzarelli, A. Cappella, E. Castoldi, S. Ripa, L. Cucca, C. Cattaneo, A. Profumo, Comparison of different swabs for sampling inorganic gunshot residue from gunshot wounds: Applicability and reliability for the de- termination of firing distance, $\mathrm{J}$. Forensic Sci. 64 (2019) 558-564, https://doi.org/10.1111/1556-4029.13870.

[67] D. Degaetano, J. Siegel, K. Klomparens, A comparison of three techniques developed for sampling and analysis of gunshot residue by Scanning Electron Microscopy Energy Dispersive X-ray analysis (SEM-EDX), J. Forensic Sci. 37 (1992) 281-300.

[68] I. Kara, The influence of different skin types on GSR sampling by tape lifting for SEM analysis, Microsc. Res. Tech. 80 (2017) 1310-1314, https://doi.org/10.1002/jemt.22942.

[69] R. Taudte, C. Roux, L. Blanes, M. Horder, K. Kirkbride, A. Beavis, The development and comparison of collection techniques for inorganic and organic gunshot residues, Anal. Bioanal. Chem. 408 (2016) 2567-2576, https://doi.org/10.1007/s00216-016-9357-7. [70] R. Schwartz, C. Zona, A recovery method for airborne gunshot residue retained in human nasal mucus, J. Forensic Sci. 40 (1995) 13845J, https://doi.org/10.1520/jfs13845j. 
[71] L. Reyes, C. Lopez, A. Barrios, C. Soto, C. Ibanez, F. Diaz, Development and application of a new nose hairs sample collection device for GSR Particles by scanning electron microscopy with energy dispersive X-ray spectroscopy (SEM-EDS), Forensic Sci. Int. 290 (2018) 42-48, https://doi.org/10.1016/j.forsciint.2018.06.029.

[72] S. Charles, M. Lannoy, N. Geusens, Influence of the type of fabric on the collection efficiency of gunshot residues, Forensic Sci. Int. 228 (2013) 42-46, https://doi.org/10.1016/j.forsciint.2013.02.022.

[73] M. Izzharif, A. Halim, U. Kalthom, C. Yew, M. Koey, Analysis of gunshot residue deposited on cotton cloth target at close range shooting distances, J. Forensic Sci. 1 (2010) 4853.

[74] W. MacCrehan, K. Ricketts, R. Baltzersen, W. Rowe, Detecting organic gunpowder residues from handgun use, Investig. Forensic Sci. Technol. 3576 (1999) 116-124, https://doi.org/10.1117/12.334522.

[75] I. Kara, S. Lisesivdin, M. Kasap, E. Er, U. Uzek, The relationship between the surface morphology and chemical composition of gunshot residue particles, J. Forensic Sci. 60 (2015) 1030-1033, https://doi.org/10.1111/1556-4029.12785.

[76] D. Merli, A. Brandone, A. Amadasi, C. Cattaneo, A. Profumo, The detection of gunshot residues in the nasal mucus of suspected shooters, Int. J. Legal Med. 130 (2016) 1045-1052, https://doi.org/10.1007/s00414-016-1375-7.

[77] S. Kage, K. Kudo, A. Kaizoji, J. Ryumoto, H. Ikeda, N. Ikeda, A simple method for detection of gunshot residue particles from hands, hair, face, and clothing using Scanning Electron Microscopy/Wavelength Dispersive X-Ray (SEM/WDX), J. Forensic Sci. 46 (2001) 15054J, https://doi.org/10.1520/jfs15054j. 
[78] A. Zeichner, N. Levin, Collection efficiency of gunshot residue (GSR) particles from hair and hands using double-side adhesive tape, J. Forensic Sci. 38 (1993) 571-584, https://doi.org/10.1520/jfs13441j.

[79] M. Trimpe, Scientific Working Group on Gunshot Residue (SWGGSR): A progress report, in: Conf. Scanning Microsc. 2011 - Adv. Microsc. Technol. Defense, Homel. Secur. Forensic, Life, Environ. Ind. Sci., Spie-Int Soc Optical Engineering, BELLINGHAM, 2011. Doi:10.1117/12.887503.

[80] R. Thompson, D. Fetterolf, M. Miller, R. Mothershead, Aqueous recovery from cotton swabs of organic explosives residue followed by solid phase extraction, J. Forensic Sci. 44 (1999) 795-804, https://doi.org/10.1520/jfs14555j.

[81] E. Pereira, C. Caceres, F. Rivera, B. Rivas, P. Saez, Preparation of molecularly imprinted polymers for diphenylamine removal from organic gunshot residues, J. Chil. Chem. Soc. 59 (2014) 2731-2736.

[82] O. Black, R. Cody, D. Edwards, J. Cizdziel, Identification of polymers and organic gunshot residue in evidence from 3D-printed firearms using DART-mass spectrometry: A feasibility study, Forensic Chem. 5 (2017) 26-32, https://doi.org/10.1016/j.forc.2017.05.003. [83] Z. Brozek-Mucha, Chemical and morphological study of gunshot residue persisting on the shooter by means of Scanning Electron Microscopy and Energy Dispersive X-Ray Spectrometry, Microsc. Microanal. 17 (2011) 972-982, https://doi.org/10.1017/s1431927611012141.

[84] E. Lindsay, M. McVicar, R. Gerard, E. Randall, J. Pearson, Passive exposure and persistence of gunshot residue (GSR) on bystanders to a shooting: Comparison of shooter and 
bystander exposure to GSR, J. Can. Soc. Forensic Sci. 44 (2011) 89-96, https://doi.org/10.1080/00085030.2011.10768144.

[85] Y. Tong, Z. Wei, C. Yang, J. Yu, X. Zhang, S. Yang, X. Deng, Y. Xu, Y. Wen,

Determination of diphenylamine stabilizer and its nitrated derivatives in smoke- less gunpowder using a tandem MS method, Analyst. 126 (2001) 480-484, https://doi.org/10.1039/b0101830.

[86] D. Perret, S. Marchese, A. Gentili, R. Curini, A. Terracciano, E. Bafile, F. Romolo, LCMS-MS determination of stabilizers and explosives residues in hand-swabs, Chromatographia. 68 (2008) 517-524, https://doi.org/10.1365/s10337-008-0746-8.

[87] D. Laza, B. Nys, J. De Kinder, A. Mesmaeker, C. Moucheron, Development of a quantitative LC-MS/MS method for the analysis of common propellant powder stabilizers in gunshot residue, J. Forensic Sci. 52 (2007) 842-850, https://doi.org/10.1111/j.15564029.2007.00490.x.

[88] A. O'Mahony, J. Wang, Electrochemical detection of gunshot residue for forensic analysis: A Review, Electroanalysis. 25 (2013) 1341-1358, https://doi.org/10.1002/elan.201300054.

[89] M.I. Halim, M. Safian, E. Elias, S. Shazali, Identification of gunshot residue from trace element by using ICP/OES, in, Comput. Informatics (2013).

[90] M. Gallidabino, F. Romolo, C. Weyermann, Characterization of volatile organic gunshot residues in fired handgun cartridges by headspace sorptive extraction, Anal. Bioanal. Chem. 407 (2015) 7123-7134, https://doi.org/10.1007/s00216-015-8874-0.

[91] R. Williamson, S. Gura, A. Tarifa, J. Almirall, The coupling of capillary microextraction of volatiles (CMV) dynamic air sampling device with DART-MS analysis for the 
detection of gunshot residues, Forensic Chem. 8 (2018) 49-56, https://doi.org/10.1016/j.forc.2018.01.005.

[92] T. Warlow, Firearms, the Law and Forensic Ballistics, 1st ed., CRC Press, London, 1996.

[93] A. Schwoeble, D. Exline, Current methods in forensic gunshot residue analysis, CRC Press, 2000.

[94] M. Rijnders, A. Stamouli, A. Bolck, Comparison of GSR composition occurring at different locations around the firing position, J. Forensic Sci. 55 (2010) 616-623, https://doi.org/10.1111/j.1556-4029.2009.01292.x.

[95] J. French, R. Morgan, J. Davy, The secondary transfer of gunshot residue: A experimental investigation carried out with SEM-EDX analysis, X-Ray Spectrom. 43 (2014) 5661, https://doi.org/10.1002/xrs.2498.

[96] J. French, R. Morgan, An experimental investigation of the indirect transfer and deposition of gunshot residue: Further studies carried out with SEM-EDX analysis, Forensic Sci. Int. 247 (2015) 14-17, https://doi.org/10.1016/j.forsciint.2014.10.023.

[97] H. Ditrich, Distribution of gunshot residues - The influence of weapon type, Forensic Sci. Int. 220 (2012) 85-90, https://doi.org/10.1016/j.forsciint.2012.01.034.

[98] G. Migeot, J. De Kinder, Gunshot residue deposits on the gas pistons of assault rifles, J. Forensic Sci. 47 (2002) 808-810.

[99] D. Greely, E. Weber, Transfer and distribution of gunshot residue through glass windows, J. Forensic Sci. 62 (2017) 869-873, https://doi.org/10.1111/1556-4029.13331. [100] I. Stone, V. DiMaio, C. Petty, Gunshot wounds: Visual and analytical procedures, J. Forensic Sci. 23 (1978) 361-367, https://doi.org/10.1520/jfs10769j.

[101] R. Nichols, Expectations regarding gunpowder depositions, Afte. 30 (1998) 91-101. 
[102] V. DiMaio, C. Petty, I. Stone, An experimental study of powder tattooing of the skin, J. Forensic Sci. 21 (1976) 376-1372, https://doi.org/10.1520/jfs10505j.

[103] Z. Brożek-Mucha, A study of gunshot residue distribution for close-range shots with a silenced gun using optical and scanning electron microscopy, X-ray microanalysis and infrared spectroscopy, Sci. Justice. (2017), https://doi.org/10.1016/j.scijus.2016.11.004.

[104] A. Gassner, M. Manganelli, D. Werner, D. Rhumorbarbe, M. Maitre, A. Beavis, C. Roux, C. Weyermann, Secondary transfer of organic gunshot residues: Empirical data to assist the evaluation of three scenarios, Sci. Justice. 59 (2019) 58-66, https://doi.org/10.1016/j.scijus.2018.08.007.

[105] A.L. Gassner, C. Ribeiro, J. Kobylinska, A. Zeichner, C. Weyermann, Organic gunshot residues: Observations about sampling and transfer mechanisms, Forensic Sci. Int. 266 (2016) 369-378, https://doi.org/10.1016/j.forsciint.2016.06.029.

[106] J. French, R. Morgan, P. Baxendell, P. Bull, Multiple transfers of particulates and their dissemination within contact networks, Sci. Justice. 52 (2012) 33-41, https://doi.org/10.1016/j.scijus.2011.05.001.

[107] S. Charles, N. Geusens, A study of the potential risk of gunshot residue transfer from special units of the police to arrested suspects, Forensic Sci. Int. 216 (2012) 78-81, https://doi.org/10.1016/j.forsciint.2011.08.022.

[108] R. Gerard, E. Lindsay, M. McVicar, E.D. Randall, A. Gapinska, Observations of gunshot residue associated with police officers, their equipment, and their vehicles, J. Can. Soc. Forensic Sci. 45 (2012) 57-63, https://doi.org/10.1080/00085030.2012.10757183. 
[109] R. Berk, S. Rochowicz, M. Wong, M. Kopina, Gunshot residue in Chicago police vehicles and facilities: An empirical study, J. Forensic Sci. 52 (2007) 838-841, https://doi.org/10.1111/j.1556-4029.2007.00457.x.

[110] M. Cook, Gunshot residue contamination of the hands of police officers following startof-shift handling of their firearm, Forensic Sci. Int. 269 (2016) 56-62, https://doi.org/10.1016/j.forsciint.2016.11.002.

[111] N. Lucas, M. Cook, K. Kirkbride, H. Kobus, Gunshot residue background on police officers: Considerations for secondary transfer in GSR evidence evaluation, Forensic Sci. Int. 297 (2019) 293-301, https://doi.org/10.1016/j.forsciint.2019.02.017.

[112] R. Gerard, M. McVicar, E. Lindsay, E. Randall, E. Harvey, The long range de- position of gunshot residue and the mechanism of its transportation, J. Can. Soc. Forensic Sci. 44 (2011) 97-104, https://doi.org/10.1080/00085030.2011.10768145.

[113] L. Fojtášek, J. Vacínová, P. Kolář, M. Kotrlý, Distribution of GSR particles in the surroundings of shooting pistol, Forensic Sci. Int. 132 (2003) 99-105, https://doi.org/10.1016/S0379-0738(03)00018-5.

[114] M. Maitre, M. Horder, K. Kirkbride, A. Gassner, C. Weyermann, C. Roux, A. Beavis, A forensic investigation on the persistence of organic gunshot residues, Forensic Sci. Int. 292 (2018) 1-10, https://doi.org/10.1016/j.forsciint.2018.08.036.

[115] T. Jalanti, P. Henchoz, A. Gallusser, M. Bonfanti, The persistence of gunshot residue on shooters' hands, Sci. Justice. 39 (1999) 48-52, https://doi.org/10.1016/s1355-0306(99)72014-9. [116] J. Kilty, Activity after shooting and its effects on the retention of primer residue, J. Forensic Sci. 20 (1975) 219-230, https://doi.org/10.1520/jfs10268j. 
[117] J. Douse, R. Smith, Trace analysis of explosives and firearm discharge residues in the metropolitan police forensic science laboratory, J. Energ. Mater. 4 (1986) 169-186.

[118] W. Zech, B. Kneubühl, M. Thali, S. Bolliger, Pistol thrown to the ground by shooter after fatal self-inflicted gunshot wound to the chest, J. Forensic Leg. Med. 18 (2011) 88-90, https://doi.org/10.1016/j.jflm.2010.11.005.

[119] L. Lagoo, L. Schaeffer, D. Szymanski, R. Smith, Detection of gunshot residue in blowfly larvae and decomposing porcine tissue using inductively coupled plasma mass spectrometry (ICP-MS), J. Forensic Sci. 55 (2010) 624-632, https://doi.org/10.1111/j.15564029.2010.01327.x.

[120] N. Lucas, M. Cook, J. Wallace, K. Kirkbride, H. Kobus, Quantifying gunshot residues in cases of suicide: Implications for evaluation of suicides and criminal shootings, Forensic Sci. Int. 266 (2016) 289-298, https://doi.org/10.1016/j.forsciint.2016.06.006.

[121] N. Lucas, H. Brown, M. Cook, K. Redman, T. Condon, H. Wrobel, K. Kirkbride, H. Kobus, A study into the distribution of gunshot residue particles in the random population, Forensic Sci. Int. 262 (2016) 150-155, https://doi.org/10.1016/j.forsciint.2016.02.050.

[122] A. Zeichner, N. Levin, Casework experience of GSR detection in Israel, on samples from hands, hair, and clothing using an auto search SEM/EDX system, J. Forensic Sci. 40 (1995) 1082-1085, https://doi.org/10.1520/jfs13881j.

[123] A. Vinokurov, A. Zeichner, B. Glattstein, A. Koffman, N. Levin, A. Rosengarten, Machine washing or brushing of clothing and its influence on shooting distance estimation, J. Forensic Sci. 46 (2001) 928-933, https://doi.org/10.1520/jfs15072j.

[124] S. Khandasammy, A. Rzhevskii, I. Lednev, A novel two-step method for the detection of organic gunshot residue for forensic purposes: Fast fluorescence imaging followed by raman 
microspectroscopic identification, Anal. Chem. 91 (2019) 11731-11737, https://doi.org/10.1021/acs.analchem.9b02306.

[125] C. Mahoney, G. Gillen, A. Fahey, Characterization of gunpowder samples using time-offlight secondary ion mass spectrometry (TOF-SIMS), Forensic Sci. Int. 158 (2006) 39-51, https://doi.org/10.1016/j.forsciint.2005.02.036.

[126] Z. Brozek-Mucha, A. Jankowicz, Evaluation of the possibility of differentiation between various types of ammunition by means of GSR examination with SEM- EDX method, Forensic Sci. Int. 123 (2001) 39-47, https://doi.org/10.1016/s0379-0738(01)00518-7.

[127] J. Coumbaros, K.P. Kirkbride, G. Klass, W. Skinner, Characterisation of 0.22 ca- liber rimfire gunshot residues by time-of-flight secondary ion mass spectrometry (TOF-SIMS): A preliminary study, Forensic Sci. Int. 119 (2001) 72-81, https://doi./10.1016/S0379$\underline{0738(00) 00421-7 .}$

[128] M. Chohra, B. Beladel, L. Ahmed, M. Mouzai, D. Akretche, A. Zeghdaoui, A. Mansouri, M. Benamar, Study of gunshot residue by NAA and ESEM/EDX using several kinds of weapon and ammunition, J. Radiat. Res. Appl. Sci. 8 (2015) 404-410, https://doi.org/10.1016/j.jrras.2015.02.012.

[129] Z. Brozek-Mucha, On the prevalence of gunshot residue in selected populations: An empirical study performed with SEM-EDX analysis, Forensic Sci. Int. 237 (2014) 46-52, https://doi.org/10.1016/j.forsciint.2014.01.020.

[130] S. Romanò, F. Degiorgio, C. Onofrio, L. Gravina, S. Abate, Characterisation of gunshot residues from non-toxic ammunition and their persistence on the shooter's hands, Int. J. Legal Med. 134 (2020) 1083-1094. 
[131] R. Costa, L. Motta, C. Destefani, R. Rodrigues, K. Santo, G. Aquije, R. Boldrini, G. Athayde, M. Carneiro, W. Romao, Gunshot residues (GSR) analysis of clean range ammunition using SEM/EDX, colorimetric test and ICP-MS: A comparative approach between the analytical techniques, Microchem. J. 129 (2016) 339-347, https://doi.org/10.1016/j.microc.2016.07.017.

[132] B. Yuksel, A. Ozler-Yigiter, T. Bora, N. Sen, Z. Kayaalti, B. Yüksel, A. Ozler-Yigiter, T. Bora, N. Sen, Z. Kayaalti, GFAAS determination of antimony, barium, and lead levels in gunshot residue swabs: An application in forensic chemistry, At. Spectrosc. 37 (2016) 164-169. [133] R. Schumacher, M. Barth, D. Neimke, L. Niewohner, Investigation of Gunshot Residue Patterns using milli-XRF-Techniques: First Experiences in Casework, in: Conf. Scanning Microsc., Spie-Int Soc Optical Engineering, Bellingham, 2010. Doi: 10.1117/12.853852. [134] M. Szynkowska, A. Parczewski, K. Szajdak, J. Rogowski, Examination of gunshot residues transfer using ToF-SIMS, Surf. Interface Anal. 45 (2013) 596-600, https://doi.org/10.1002/sia.5142. 


\section{CHAPTER 4}

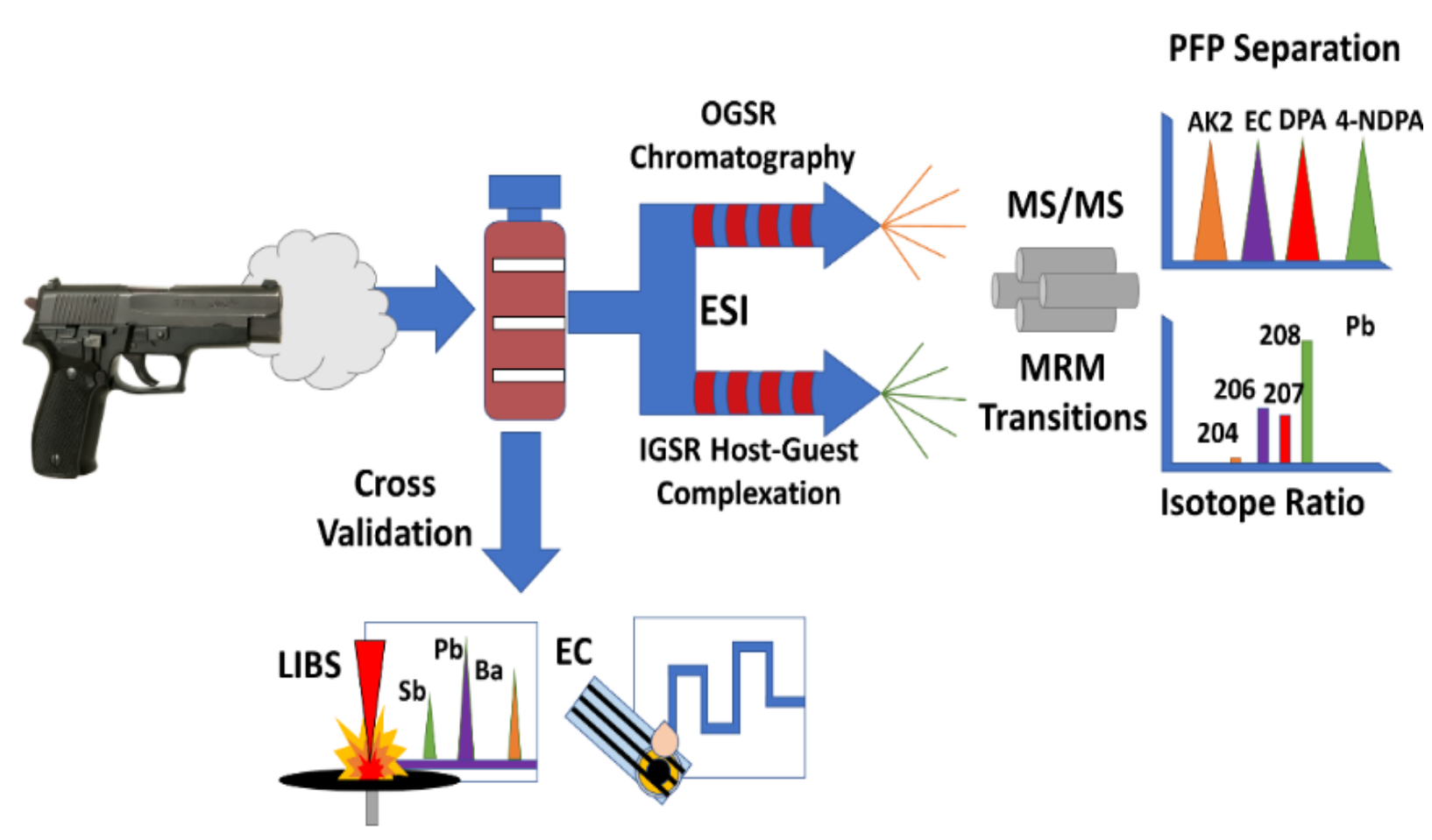

Detection of Organic and Inorganic Gunshot Residues from Hands using Complexing Agents and LC-MS/MS 
Overview: Gunshot residue (GSR) refers to a conglomerate consisting of both organic molecules (OGSR) and inorganic species (IGSR). Historically, forensic examiners have focused only on identifying the IGSR particles by their morphology and elemental composition. Nonetheless, modern ammunition formulations and challenges with the GSR transference (such as secondary and tertiary transfer) have driven research efforts for more comprehensive examinations, requiring alternative analytical techniques. This study proposes the use of LC-MS/MS for chromatographic separation and dual detection of inorganic and organic residues. The detection of both target species in the same sample increases the confidence that chemical profiles came from a gun's discharge instead of non-firearm-related sources. This strategy implements supramolecular molecules that complex with the IGSR species, allowing them to elute from the column towards the mass spectrometer while retaining isotopic ratios for quick and unambiguous identification. The macrocycle (18-crown-6-ether) complexes with lead and barium, while antimony complexes with a chelating agent (tartaric acid). The total analysis time for OGSR and IGSR in one sample is under 20 minutes. This manuscript expands from a previous proof-of-concept publication by improving figures of merit, increasing the target analytes, testing the method's feasibility through a more extensive set of authentic specimens collected from the hands of both shooters and nonshooters, and comparing performance with other analytical techniques such as ICP-MS, electrochemical methods and LIBS. The linear dynamic ranges (LDR) spread across the low ppb range for OGSR (0.3-200 ppb) and low ppm range (0.1-6.0 ppm) for IGSR. The method's accuracy increased overall when both organic and inorganic profiles were combined.

The following chapter is an adaptation of a previously published article (O2021: William Feeney, Korina Menking-Hoggatt, Courtney Vander Pyl, Colby E. Ott, Suzanne Bell, Luis Arroyo, Tatiana Trejos. Detection of Organic and Inorganic Gunshot Residues from Hands using 
Complexing Agents and LC-MS/MS. Analytical Methods. Published online June 2021, 13, 3024 https://doi.org/10.1039/D1AY00778E

\subsection{Introduction}

\section{Trace Analysis Disciplines - Gunshot Residue}

\subsubsection{Background}

Forensic trace examiners investigate a broad range of materials, including tape, hair, fibers, paint, fire debris, gunshot residues, and many others [1]. This type of evidence occurs from a physical transference event between two or more objects or persons and was famously coined by Edmond Locard stating "every contact leaves a trace" [1]. One of the most studied and debated trace materials within the forensic community is residue released during the discharge of a firearm, due to its complex transfer and persistence mechanisms.

Gunshot residue (GSR) comprises two main components, organic (OGSR) and inorganic (IGSR), which arise from different locations within the ammunition. The OGSR compounds originate from the propellant and lubricant, whereas IGSR particulates emanate from the primer, bullet, and cartridge casing. After a deflagration event, those analytes can be dispersed and spread onto various surrounding surfaces, including hair, clothing, and hands. Due to the constituents' nature and various environmental factors, proficient collection and storage of the samples are essential to preserve the GSR compounds and increase the likelihood of detection. Typical indicators for IGSR are $\mathrm{Pb}, \mathrm{Ba}$, and $\mathrm{Sb}$ which are formed from the initial products lead styphnate $\left(\mathrm{C}_{6} \mathrm{HN}_{3} \mathrm{O}_{8} \mathrm{~Pb}\right)$, barium nitrate $\left(\mathrm{Ba}\left(\mathrm{NO}_{3}\right)_{2}\right)$, and antimony trisulfide $\left(\mathrm{Sb}_{2} \mathrm{~S}_{3}\right)$. Some of the more common OGSR analytes are diphenylamine (DPA), nitroglycerin (NG), ethyl centralite (EC), and 2,4-dinitrotoluene (2,4-DNT) [2,3]. Other compounds monitored, primarily formed by the combustion event and degradation of DPA, include 2-dinitrodiphenylamine (2-NDPA), 4- 
nitrodiphenylamine (4-NDPA), and N-nitrosodiphenylamine (N-NDPA). These compounds' functional roles vary from detonation or blasting agents (explosives, oxidizers, fuel) to binding and performance materials (stabilizers and plasticizers) $[2,4]$.

Recently, manufacturers have introduced variants of ammunition labeled as "green," nontoxic, heavy-metal-free, or lead-free. These products incorporate different starting materials to achieve similar results to traditional ammunition while reducing exposure of the shooter and environment to harmful heavy metals. Although this type of ammunition is not widely observed in casework yet, its emergence has required researchers to characterize and adapt interpretation criteria for non-toxic primers [5-8].

\subsubsection{Inorganic Particulate Analysis}

Under the ASTM E1588-20 guideline, the standard instrument for identifying GSR is Scanning Electron Microscopy Energy Dispersive X-Ray Spectrometry (SEM/EDS) [9]. This nondestructive technique identifies the presence or absence of GSR based on the morphology and elemental composition of a single particle. The current guideline provides instructions for the proper identification of IGSR and uses terminology to indicate the degree of confidence in the identification of IGSR. Currently, SEM/EDS remains the only confirmatory standard for GSR [9]. The discrimination power of identifying particulates is founded on the elemental profiles categorized by three levels of discriminating power alongside distinctive spheroid morphologies. The terms used to describe the confidence in differentiating GSR from other non-GSR environmental sources are: "characteristic," "consistent," and "commonly associated particles".

Even though SEM/EDS is efficient in characterizing micron-sized inorganic particles, the method is not compatible with further sequential examination for OGSR, as factors such as high vacuum conditions, operating parameters, and compound volatility can cause substantial analyte 
loss. Hence if SEM/EDS analysis is to be used in conjunction with another analysis technique, the OGSR constituents must be collected first. Another disadvantage is that the analysis time for SEM/EDS typically takes 2-8 hours per sample, depending on the sample's nature and instrumental configurations. Additionally, hand residues associated with occupations such as electricians, welders, and mechanics can lead to false-positives and higher error rates [10]. A recent study has proposed a solution to OGSR loss by first analyzing the OGSR from the stub using mild solvent extraction with UHPLC-ESI-MS/MS followed by IGSR examination from the same SEM collection stub [11]. These authors demonstrated that by using gentle mechanistic motions from a pipette, the IGSR particulates are not significantly disturbed.

Current advancements in instrumentation techniques have shown great promise for evaluating GSR evidence. Instruments such as LIBS, LA-ICP-MS, and TOF-SIMS offer non- or minimally destructive analysis, small particle detection, and the capacity to produce high-quality images with multielement or isotopic composition information [12-14]. High-resolution instruments such as ICP-MS can provide additional isotopic and elemental information in the low part-per-billion $(\mathrm{ppb})$ range $[15,16]$.

\subsubsection{Organic Compound Analysis}

Unlike IGSR, there is no established guideline for characterizing and interpreting the data for the OGSR constituents. However, some initial efforts to classify and select relevant OGSR constituents are based on their prevalence in the environment, expected occurrence due to its use outside the ammunition market, and existing knowledge of published compounds, mainly by GCMS and LC-MS methods [17]. These techniques provide sufficient distinctions between compounds such as Kovats' retention indices, chromatographic separation, fragmentation pathways, and mass spectral data libraries. One difference between OGSR and IGSR analysis is 
extensive sample preparation. Extraction protocols deviate from the ASTM E1588-20 guideline by implementing aqueous buffers or organic solvents, such as methanol $(\mathrm{MeOH})$ or acetonitrile $(\mathrm{ACN})$. Depending on the chemical composition, specific methodologies may more effectively characterize and detect OGSR constituents by exploiting properties like polarity or volatility. Because of parameters and instrumentation flexibilities, factors like total analysis times, sensitivities, and spectral information are more prone to fluctuate. This variation and capabilities, in turn, are the main challenges for forensic laboratories to create and develop an inclusive, standard guideline for OGSR. Moreover, detection limits of some GC-MS configurations may not be applicable to concentrations typically found in GSR specimens.

\subsubsection{Combined Analysis Methodologies}

Because of the additional and valuable evidentiary information gained from OGSR analysis, there has been a shift to analyze both IGSR and OGSR components from a single sample. Techniques including electrochemistry, FTIR, and Raman spectroscopy offer rapid, cost-effective multicomponent analysis alternatives, but lack the sensitive/selective power compared to mass spectrometry $[18,19]$. Therefore, various research groups combine screening and confirmatory methodologies to increase confidence in the results $[12,13,20-32]$. Such combinations include LIBS with electrochemistry, LC-MS/MS with SEM/EDS, Raman spectroscopy with LA-ICP/MS, and CMV-GC/MS with LIBS [11,12,30,33,34].

LIBS, electrochemistry, and Raman spectroscopy facilitate sample screenings for quick characterizations with minimal sample preparation, and the sample remains almost unaltered. Additionally, the GSR stub used in these methods is compatible with the confirmatory (SEM/EDS) analysis, allowing further sample manipulation. 
Extraction for OGSR constituents use common solvents (methanol and acetonitrile), whereas IGSR elemental identification requires a more rigorous heavy metal digestion (nitric acid and hydrochloric acid). Therefore, methods like capillary electrophoresis and LC-MS/MS are classified as destructive methods [35,36]. However, if these methods were incorporated in a comprehensive workflow, the morphology analysis required can be conducted after organic extractions and instrumental analysis (via capillary electrophoresis, DESI, or LC-MS/MS) and before the losing OGSR due to vacuum conditions via SEM/EDS [11,37-40].

Due to the structure of IGSR particles, further sample manipulations are required to identify and quantify samples properly via LC-MS/MS. Ideally, these chemical analyses should provide unique spectral signatures without altering the core information of the analyte of interest. This consideration led to the investigation of complexation chemistry. Host-guest chemistry applies larger organic molecules to self-assemble and form complexes called metal-ligand (M-L) complexes. Macrocycles like 18-crown-6-ether (18C6) encapsulate metal ions through noncovalent, electrostatic interactions [35,41,42]. Several benefits from this interaction include transportation through both the column and mass analyzer, an extensive range of metal analytes, and retaining of natural isotopic abundance patterns. Other molecules like EDTA and tartaric acid employ a different strategy for cation binding, known as chelation. Chelation involves the formation of physical coordinate bonds between a ligand and a single central atom. Although this can provide similar benefits as host-guest chemistry, more extensive factors like $\mathrm{pH}$ must be considered.

The goal of this work addresses key points which include: 1) validate our previous proofof-concept study by enhancing figures of merit such as detection limits (LOD), quantitation limits (LOQ), bias, and expanding the evaluation of performance rates with authentic specimens [35], 2) 
investigate interactions and expand upon antimony detection, and 3) establish an identification criterion for a "positive" GSR sample based on baseline authentic samples. This work also evaluates two substrates, including tesa ${ }^{\circledR}$ Tack and the traditional carbon adhesive tape. Furthermore, we investigate various sample types, including samples collected from shooters, nonshooter skin backgrounds, and post-shooting activity.

\subsection{Materials and Methods}

\subsubsection{Consumables}

Optima ${ }^{\circledR}$ LC/MS grade methanol $(\mathrm{MeOH})$, acetonitrile $(\mathrm{ACN})$, and water $\left(\mathrm{H}_{2} \mathrm{O}\right)$, all containing $0.1 \%$ formic acid (FA), were obtained from Thermo-Fisher Scientific (Watham, MA) and used either as extraction solvents or mobile phases. Standard (neat) organic constituents used in this study included: akardite II (AK 2), ethyl centralite (EC), methyl centralite (MC), diphenylamine (DPA), N-nitrosodiphenylamine (N-NDPA), 2-nitrodiphenylamine (2-NDPA), and 4-nitrodiphenylamine (4-NDPA), all obtained from Sigma-Aldrich and Accustandard® (New Haven, CT). Calibration curves were generated from working stock solutions and diluted in $\mathrm{MeOH}$ ( $\geq 95 \%)$. Complexing agents 18-crown-6-ether (18C6) and tartaric acid (TT) were purchased from Sigma-Aldrich (St. Louis, MO 99\% purity) and diluted using $\mathrm{MeOH}$ and water, respectively. Metal IGSR standards used to form complexes utilized ICP-MS metal standards (VHG Laboratories, Manchester, $\mathrm{NH})$ and methanol. Micro-bulk digestions utilized nitric $\left(\mathrm{HNO}_{3}\right)$ and hydrochloric ( $\mathrm{HCl}$ ) acids (ultrapure grade, Thermo-Fisher Scientific, Waltham, MA). Two internal standards were used throughout the study including deuterated diphenylamine- $\mathrm{d}_{10}\left(\mathrm{D}_{10}-\mathrm{DPA}\right)$ which was purchased from CDN Isotopes (Quebec, Canada) and used as the internal standard for the organic molecules and thallium (Tl) for the IGSR (VHG Laboratories, Manchester, NH) and complexing agents. 


\subsubsection{Firearms, ammunition, and protocols}

Hands from the hands of volunteers, both from shooters and non-shooter background individuals were collected under our institutional IRB protocol \#1506706336 using carbon adhesive. Aluminum SEM stubs were prepared with two layers of carbon adhesive tape and covered with plastic stub storage containers (Ted Pella, Inc.).

In this study, three different firearms were used for the collection of authentic shooter samples: $9 \mathrm{~mm}$ Springfield XD9 semi-automatic pistol, 40 caliber Springfield XD40 semiautomatic pistol, and a Taurus 357 Magnum revolver. The ammunition was either loaded by the manufacturer (Remington ${ }^{\circledR}$, Blazer ${ }^{\circledR}$, Federal ${ }^{\circledR}$ ) or in-house (Winchester ${ }^{\circledR}, \mathrm{CCI} ®$ ). Each specialty ammunition consisted of brass Starline ${ }^{\mathrm{TM}}$ cartridge cases loaded with either Winchester ${ }^{\circledR}$ or $\mathrm{CCI}{ }^{\circledR}$ small pistol primers, Winchester ${ }^{\circledR} 231$ propellant, and Speer® 9mm total metal jacketed bullets. All firing events were performed at the indoor WVU ballistics laboratory and the respective analysis conducted at the Oglebay Hall research building.

Before each session, the working areas were cleaned and covered with butcher paper, and samples manipulated with disposable nitrile gloves. To minimize cross-contamination, our research team established a workflow within five separate laboratory areas. One room is dedicated to preparing the sampling stubs before collection, which is located in the building within our trace laboratory. After collection, the samples are stored in a refrigerator until extraction, which is conducted in a separate laboratory room equipped with dedicated hoods and benchtops. Finally, instrumental analysis is performed on the third floor for the LC-MS/MS, ICP-MS, LIBS, on the second floor for EC, and on the ground level for the SEM-EDS examinations.

Moreover, the shooting range is located in another building a few miles apart from the research laboratory, and firearms and ammunition are stored in dedicated safety rooms away from 
research spaces. Within the ballistics lab, firearm discharge occurs inside the shooting range, while collection from the individual's hands is done on the annexed laboratory. Also, working areas are cleaned daily before any sample preparation or analysis. The team members have specific scheduled roles to avoid that an individual who has recently fired or manipulated a firearm enters the laboratory areas. The collection team members and shooters dressed in Tyvek® suits for additional protection from carryover.

Our standard operating procedure includes several reagent blanks and negative control samples to monitor any potential contamination. Reagent blanks are analyzed between each sample to monitor any carryover or unexpected contamination. Negative controls are prepared from clean carbon stubs that had not been used for hands collection, but that undergo the same extraction protocol as the respective sample batch. In addition, negative controls from the hands of the collecting individuals undergo the whole analytical process from collection to extraction to analysis. Negative control samples are obtained from the hands of the collection team at the beginning and end of each collection session.

The shooters discharged five consecutive shots inside the WVU ballistics range. Although the number of shots fired per shooter varies on a case-to-case basis, the number of discharges were chosen in this study based on previously reported literature as well as the casework experience of colleagues, who reported that typically a shooter fires 3-5 shots when using revolvers and pistols in criminal activities $[10,43]$. After firing 5 shots, the firearm was cleared and placed with the range officer. The shooters then proceeded to the collection laboratory, where both left- and right hands (palm and back) were stubbed 15 times in the areas from the index finger to the thumb. Each firing event generated four samples, which is outlined in Figure 4.1. After sample collection, the shooters washed their hands and repeated the process. After the firing session concluded, the 
collection team obtained additional negative control samples to test potential secondary transfers or unintended contamination. Once all samples were stored, the Tyvek® suits, parchment paper, and gloves were discarded.

For the activity sessions, three different activities were performed after 5 successive discharges including hand rubbing, hand sanitizer application, and running. For the first activity, the subjects clasped both hands together and vigorously rubbed for approximately 30 seconds. After that time, the collectors proceeded to stub the subjects index and thumb areas. The second activity followed the similar motion as the first activity except for hand sanitizer application. For the final activity, the subjects exited the range and proceeded to run for approximately 60 seconds outside of the range. The collection team then proceeded to stub theirs hands after they entered the ballistics laboratory.

\subsubsection{LC-MS/MS methods}

\subsubsection{Mass Spectrometry confirmation via LC-MS/MS and Inorganic Isotopic Pattern Identification using Q-Exactive Orbitrap}

Before initial authentic sample collection, both OGSR and IGSR standards were subjected to rigorous characterization and optimizations using different mass analyzers. Both an Agilent 1290 Infinity II liquid chromatography coupled to a 6470-triple quadrupole (QQQ) mass analyzer and a Thermo Fisher Scientific Q-Exactive ${ }^{\circledR}$ orbitrap analyzed compounds using flow-injection analysis (FIA). The OGSR only utilized the 6470 QQQ and the Agilent Optimizer® software to determine fragmentation patterns and compared them to the NIST mass spectral database. On the other hand, due to the more complex nature of the M-L structures, IGSR compounds were further analyzed by Q-Exactive Orbitrap. The Q-Exactive Orbitrap was utilized to observe the isotopic distribution of the inorganic elements as present when exposed to the crown complex. No 
chromatographic analysis was conducted since the Orbitrap was utilized as a confirmation tool using direct infusion approach. The observation of the natural abundances for studied elements serve as confirmation of their presence in the complex agent. Further confirmation was performed with the chromatographic analysis using the tandem mass spectrometer.

\subsubsection{LC-MS/MS flow and column conditions}

An Agilent 1290 Infinity II liquid chromatography housing an Agilent pentafluorophenyl (PFP) Poroshell@ 120 column $(2.7 \mu \mathrm{m} 2.1$ x $50 \mathrm{~mm})$ separated OGSR compounds. The binary flow parameters consisted of water with $0.1 \% \mathrm{FA}(A)$ and acetonitrile with $0.1 \%(B)$ with a flow rate of $0.350 \mathrm{~mL} / \mathrm{min}$. Initial conditions were $80 \% \mathrm{~A} / 20 \% \mathrm{~B}$ and ramping to $5 \% \mathrm{~A} / 95 \% \mathrm{~B}$ for nine minutes (Table S4.1). Additionally, the source conditions are described in Table S4.2. The total injection volume was $1.0 \mu \mathrm{L}$.

For the inorganics, a Hamilton PRP-X100 cation exchange guard column $(10 \mu \mathrm{m} 2.1$ x 33 $\mathrm{mm}$ ) was added to the LC system. The crown ether complexes eluted from the column using an isocratic flow at $90 \% A / 10 \% B$ in positive electrospray (ESI) conditions. At 4 minutes, the source polarity switched to negative ESI (ESI-) mode, and the composition of mobile phases switched to $98 \% A / 2 \% B$ for the tartaric acid complexes. The injection volume for the IGSR method was $10 \mu \mathrm{L}$.

\subsubsection{LC-MS/MS mass spectral analysis}

Two classifications are used for MRM identification for precursor ions: quantifiers and qualifiers ions. Quantifier ions represent the most intense fragment ions formed from ionization used for quantitation. Qualifier ions are comprised of other abundant ions to differentiate from possible interferences present in authentic samples. For the self-assembled metal-ligand complexes, the product ions were further monitored based on the bare metal's naturally occurring isotopes (discussed in detail below). 


\subsubsection{Performance checks}

Before every worklist or sequence run, the QQQ was subjected to two performance checks, including a CheckTune and calibration curve for instrument and column monitoring. This ensured high-quality data collection and characterizations for potential signal loss. The calibration curves consisted of nine levels spanning from 1-200 ppb and 0.1-6 ppm for OGSR and IGSR, respectively. Several method blanks consisting of methanol (OGSR) and acid mixture subjected to the entire digestion process (IGSR) allowed for carryover monitoring to establish the data acquisition method performance. Furthermore, negative controls from the hands of non-shooters are monitored as explained in Section 4.2.2. Positive controls consisting of standard mixtures of known composition and concentration and in-house characterized micro-particle GSR standards were monitored to check for extraction and detection efficiency before the authentic samples' batch.

\subsubsection{LC-MS/MS validation with standards and authentic samples}

Numerous analytical guidelines describe procedures to evaluate and increase the overall effectiveness of a validation method. After careful consideration, the Eurachem guideline The Fitness for Purpose of Analytical Methods [44] was chosen due to its broad expanse of analytical practices (definitions in Table $\mathbf{S 4 . 3}$ ). This study estimated the figures of merit such as analytical selectivity, LOD, LOQ, sensitivity, working range, and bias. Calibration curves were run in five replicates in three different days. Also, we evaluated performance rates based on a population of hands from volunteer shooters and non-shooters that resemble casework samples. Performance rates included sensitivity, selectivity, and accuracy.

To further address the bias of this method, a primer-only standard was used to evaluate the percent recovery of the micro-bulk digestion method. This was compared to the validated ICP-MS 
method [45] to further compare the concentrations observed in the LC-MS/MS (see Section 4.4.4). The parameters of the ICP-MS parameters and compared digestion methodology are further detailed by Menking-Hoggatt et al. [45].

\subsubsection{Comparisons with various analytical methods}

Our research group has published various innovative methods that can complement current practice. The techniques have been selected to be compatible and complementary to SEM-EDS while providing faster and informative data for case triaging. These include LIBS and electrochemistry, which have demonstrated overall accuracy greater than $90 \%$ in several population sets of authentic hand specimens. A goal of the methods, or a combination of them, is to become adoptable in the laboratory and on-site crime scene settings for more effective and streamline decision-making processes. In this study, LC-MS/MS is presented as a powerful tool to provide confirmatory information by dual detection of OGSR and IGSR components. To compare the method's performance, the results from authentic sets are compared to practically nondestructive LIBS and ED methods, and the feasibility of using LC-MS/MS alone or in combination with fast pre-screening tools is evaluated. Detailed information on the methods is described below. On the other hand, this study aims to compare the performance of tesa ${ }^{\circledR}$ Tack polymer versus traditional carbon stubs for the collection of IGSR and OGSR. The LC-MS/MS results were compared to ICP-MS, as detailed below, to evaluate the recovery efficiency.

\subsubsection{LIBS analysis}

The LIBS analysis of the stubs was conducted using a $266 \mathrm{~nm} 10 \mathrm{~ns}-\mathrm{Nd}$ : YAG LIBS system (J200 Tandem Model, Applied Spectra, CA). The system was operated with a six-channel CzernyTurner spectrometer with a spectral range from 190 to $1040 \mathrm{~nm}$ and a CCD-based broadband detector. The method fires two laser shots per ablation spot (100 $\mu \mathrm{m}$ laser spot size), leaving the 
sample practically intact for further analysis. The ablation is repeated 25 times per stub, collecting 25 spectra per sample with spatial (x, y location) and spectrochemical information of multiple emission lines in under 1.5 minutes. More detailed information on the optimized parameters can be found in the previous publication. After LIBS analysis, the same stub was submitted to electrochemical testing (see Section 4.4.2.), followed by LC-MS/MS.

\subsubsection{Electrochemical analysis}

Square-Wave Anodic Stripping Voltammetric (SWASV) was used for the analysis of the stubs using the parameters described in Ott et al., [46] disposable screen-printed carbon electrodes (SPCEs) model type DRP-110 (Metrohm DropSens, USA) and an Autolab PGSTAT128N potentiostat and the NOVA software (version 2.1.4, from Metrohm USA). Simultaneous electrochemical detection of IGSR and OGSR compounds was achieved in under 5 minutes per sample. Due to the non-destructive nature of EC, the sample aliquots were analyzed by LC-MS/MS in Section 4.4.4.

\subsubsection{Extraction and collection of authentic samples for LC-MS/MS and multi-technique approach}

Samples were collected following the ASTM E1588-20 guideline [9] and stored in a $4^{\circ} \mathrm{C}$ freezer to prevent sample loss and cross-contamination. A total of four samples are generated from a single firing event, including both dominant and non-dominant hands. Conversely, only two stubs were collected from the hands of the collectors (negative controls), one per hand. The collection areas typically stubbed include the index finger, thumb region, and the webbing between them, palm and back of the hands.

For LC-MS/MS analysis, six consecutive washes of methanol are taken directly onto the surface substrate $(6 \times 50 \mu \mathrm{L})$ for a total of $300 \mu \mathrm{L}$. The aliquots were transferred to a $0.2 \mu \mathrm{m}$ 
filtration vial and centrifuged for three minutes. The washings were aliquoted into a second filter apparatus $(0.45 \mu \mathrm{m})$ to ensure no residual polymer or adhesive surfactants could suppress analyte signals. For OGSR analysis, $100 \mu \mathrm{L}$ of the filtered solution was aliquoted into a separate vial, and $\mathrm{D}_{10}$-DPA was spiked to yield a final concentration of $150 \mathrm{ppb}$. The remaining $200 \mu \mathrm{L}$ was dried under a steady stream of $\mathrm{N}_{2}$ until $\sim 50 \mu \mathrm{L}$ remained in the centrifuge vial. A $500 \mu \mathrm{L}$ acid mixture of 2:1 concentrated acid solution $\left(\mathrm{HCl}: \mathrm{HNO}_{3}\right)$ was applied to the substrate surface and gently pipetted on the carbon adhesive surface to remove any remaining particulates. This acid mixture was added to the $50 \mu \mathrm{L}$ remnant of the organic wash and heated at $85^{\circ} \mathrm{C}$ for 10 minutes inside a sterile, plastic centrifuge tube for micro-bulk digestion. After 10 minutes, the solution cooled, and a high concentration of the complexing agents $(1 \mathrm{ppm})$ was added to that mixture to ensure pairings of the solubilized particulates with host agents could self-assemble and form complexes.

Also, to test the capability of the LC-MS/MS method to be applied as a confirmatory tool after fast screening. A subset of samples was analyzed by a newly developed approach in our group by LIBS and electrochemistry, followed by LC-MS/MS confirmation on the same stub. LIBS first characterized the four stubs for the IGSR elemental information. Micro-spatial information was obtained about the samples, and multiple wavelengths were monitored GSR elements of interest using a $\sim 15 \mathrm{~mJ}$ pulse laser as per a previously validated method [45,47]. After LIBS analysis, the samples were extracted using organic solvents and aqueous buffers for electrochemical methods. First, $100 \mu \mathrm{L}$ of acetonitrile with gentle mechanical pipette washings was applied to the ablated area of the stub. This extract was split into $50 \mu \mathrm{L}$ aliquots in $650 \mu \mathrm{L}$ microcentrifuge tubes. One aliquot was dried under a steady stream of $\mathrm{N}_{2}$ gas and the other was saved for LC-MS/MS analysis. While the drying was occurring, $50 \mu \mathrm{L}$ of a $0.1 \mathrm{M}$ acetate buffer ( $\mathrm{pH} 4.0$ ) was distributed on the stub's surface with gentle mechanical washings. This aliquot was used to reconstitute the dried 
down organic fraction. After vortexing, the combined $50 \mu \mathrm{L}$ drop, encompassing the organic and inorganic fractions, was placed on a carbon electrode for electrochemical analysis by square-wave voltammetry and monitored for both OGSR and IGSR simultaneously [12,46,47].

After electrochemistry, the organic extract was run on LC-MS/MS to confirm and detect any OGSR. After analyzing the organic samples, the combined acetonitrile and buffer samples was subjected to the micro-bulk method mentioned above. The complexing agents were added to the mixture after cooling. Each extract was injected once for each hand area, for four replicates per individual (left, right, palm and back). Figure 4.1 illustrates the entire combined extraction and analysis process from a single firing event and the same sample.
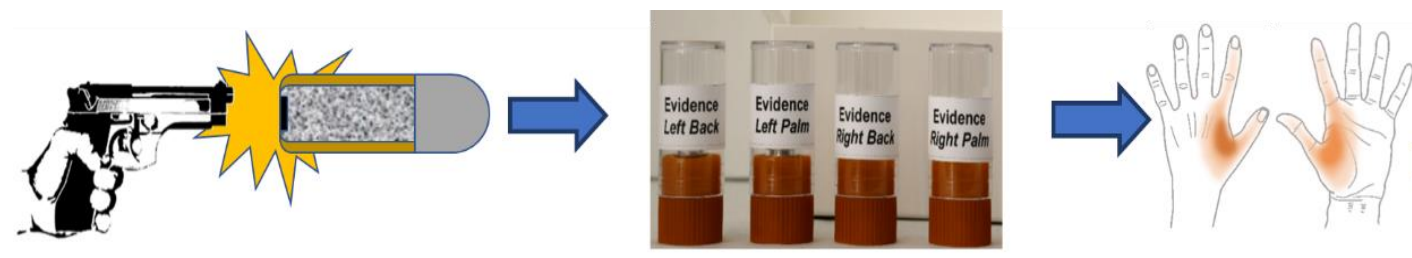

Screening Techniques
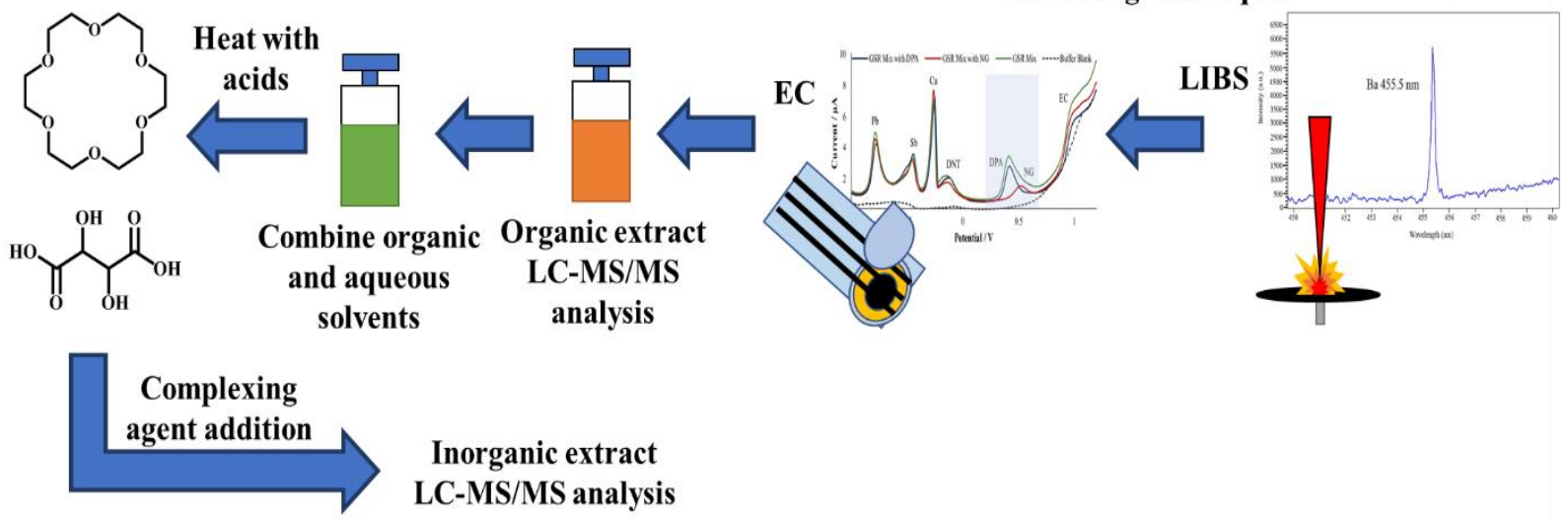

Figure 4.1: Breakdown of the extraction and analysis process, including cross-validation from screening techniques using a single sample set from a discharge event. From one firing session, each hand is stubbed in the areas highlighted in orange to generate four samples. LIBS first characterizes all four stubs using elemental analysis obtained from the IGSR particulates. The same samples are then followed by electrochemical methods where both IGSR and OGSR are both monitored. The organic extracts from those tests are then tested via LC-MS/MS for OGSR separation and then micro-bulk digestion and complexation for IGSR characterization. 


\subsubsection{ICP-MS analysis for recovery study of collection substrates}

Two digestion methods were compared for collection efficiencies of the substrates using ICP standards and the WIN p-GSR micro-particle standard. Both digestions consisted of utilizing heavy acids $\left(\mathrm{HNO}_{3}\right.$ and $\left.\mathrm{HCl}\right)$ and heating for different times. The surface digestion utilized multiple washings for a final volume of $500 \mu \mathrm{L}$. These washings were then heated at $85^{\circ}$ for 10 minutes. The ICP-MS protocol fully submerged the substrates in 10\% nitric acid (ICP-MS grade) at $80^{\circ}$ for 60 minutes. A hot block acid digestion (Environmental Express, SC) followed by ICPMS method was used as comparative bulk analysis to characterize and quantify the elemental composition of the GSR residues on carbon and tesa® Tack stubs.

An ICP-MS instrument (Agilent 7800, Santa Clara, CA) with a MicroMist nebulizer and double pass quartz Scott-type spray chamber was used for the analysis using the parameters reported by Menking-Hoggatt et al. [78]. After these digestions, both acid mixtures were diluted to $2 \%$ nitric acid mixtures where they would be compared to a calibration curve ranging from 0 $300 \mathrm{ppb}$ within the same matrix of $2 \%$ nitric acid. For the ICP standards and the WIN p-GSR standard, we performed three replicates across three days $(\mathrm{n}=9)$. We spiked Indium at $150 \mathrm{ppb}$ for the internal standard and performed quality control (QC) runs at two concentrations (25 and 50 ppb) to monitor any loss of response for the instrument. Additionally, we performed method blanks where no analytes were spiked onto the substrate surfaces. We applied the acids to the bare substrates to monitor any potential enhancements or suppression of signal.

\subsection{Results and Discussion}

Identification, characterization, and optimization of OGSR and IGSR 


\subsubsection{OGSR: Chromatography, CID experimentation, and observations}

During the optimization process, the organic compounds were independently infused into the source and the mass analyzer. Both precursor and product ions were optimized using various source conditions to find transitions equivalent to literature values $[10,11,48,49]$. A pentafluorophenyl (PFP) column was used to achieve a reasonable separation of OGSR analytes for a total run time of fewer than five minutes. One benefit of incorporating this specialized column is its robustness and versatility to use traditional mobile phases used by $\mathrm{C}_{18}$. The primary advantage of the PFP column versus traditional $\mathrm{C}_{18}$ silicate columns is the composition of the stationary phase regarding the functional group. This column's interaction mechanism utilizes phenyl rings for $\pi-\pi$ interactions and hydrogen bonding for improved selectivity of traditional OGSR constituents. The main differences between the previous proof-of-concept study [35] and the presented work are the improved chromatography and increased analyte observation. In the previous publication, DPA and EC coeluted, making it difficult to resolve chromatographically. However, the different column environment and structure allowed for a clear separation between retention times and elution order as illustrated by Figure 4.2. 


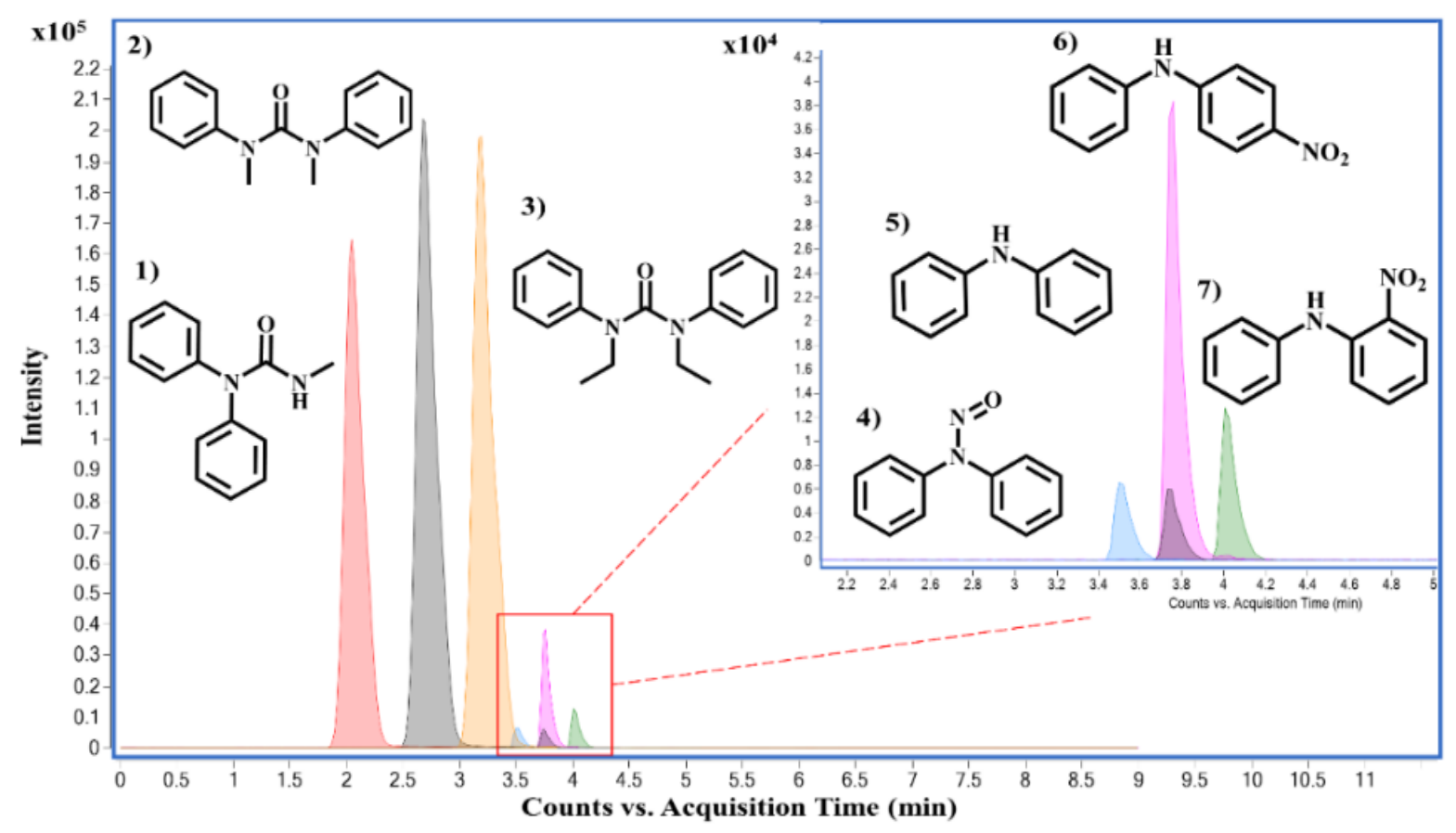

Figure 4.2: Chromatography separation of the seven OGSR compounds using the pentafluorophenyl column. Standards of OGSR are eluted (minutes) based on their polarities and interactions with the pentafluorophenyl column in the following order: 1) Akardite II (2.00 min), 2) Methyl Centralite (2.67 min), 3) Ethyl Centralite (3.19 min), 4) N-Nitrosodiphenylamine (3.49 $\mathrm{min})$, 5) Diphenylamine (3.75 $\mathrm{min})$, 6) 4-Nitrodiphenylamine (3.76 min), and 7) 2Nitrodiphenylamine (4.00 min). Each OGSR compound was measured with an electrospray source in $(+)$ mode.

There are over one hundred OGSR compounds that have been reported in different studies.

However, we selected seven major compounds (Figure 4.2) that are more indicative of gunshot residue as they are not common in the environment nor prevalent in non-shooter populations [10].

One advantage of LC-MS methods is they can be easily expanded to additional GSR analytes as long as they are compatible with the ionization requirements. Amongst the constituent list is AK2, which differs from DPA by an amide addition, and 2-NDPA, a positional isomer to 4-NDPA. These OGSR additions to our method were made due to shifts in modern propellant formulations and potential degradation and deflagration entropy of more prominent indicators such as DPA. Interestingly, compounds like MC and EC or 4-NDPA and 2-NDPA, which only differ by methyl groups or the nitro-group position, can be identified by elution time alone. 
Source conditions were also optimized for improved sensitivities of the OGSR compounds. These conditions were modeled after Ali et al., who utilized a similar LC-MS/MS system [33]. However, the discernable difference between the two methodologies is the increased transitions for all compounds in our method, barring 2-NDPA, and the lowered injection volume ( $1 \mu \mathrm{L}$ vs. 5 $\mu \mathrm{L})$. As expected, most OGSR analytes were suitable to ESI(+). However, it is important to note that two typical OGSR markers, nitroglycerin (NG) and 2,4-dinitrotoluene (2,4-DNT), were not observed using electrospray ionization in positive mode. Several technical publications documented this observation using the same instrument source setup: the Agilent Jet Stream ${ }^{\circ}$ ESI (AJS-ESI) [50]. The limitation in ionization is potentially due to the chemical environment conditions of the analyte, which showed very low efficiency in positive ionization. Although ESI (-) mode can detect these compounds, it requires the assistance of signal enhancers to promote adduct formation (salt forms) [51]. These anionic additives include acetates, chlorides, iodides, and other nitrate sources [51]. Varying sources like atmospheric pressure chemical ionization (APCI-), UV detectors, and recently direct sample analysis (DSA) can circumvent signal problems and observe these compounds easily [52-55]. The addition of $\mathrm{Na}$ or $\mathrm{K}$ to the medium may be useful in some instances, but it was not considered a viable option in our study since these two elements are expected to be widely distributed in the environment and in the skin surfaces sampled for GSR. Also, $\mathrm{Na}$ and $\mathrm{K}$ are easy to ionize and may cause in-source ionization competition that can affect the efficacy of the metal clusters response. Unfortunately, these options require either dopants or other sources (i.e., APPI) which may lead to additional costs and complications to a forensic laboratory. Therefore, a decision was made to maintain the method's simplicity by using ESI in positive mode while monitoring seven key OGSR components. Alternatively, our approach can 
incorporate rapid screening with electrochemical methods, one can identify and characterize these two troublesome compounds (NG and 2,4-DNT) [12].

\subsubsection{IGSR: Formation and identification of M-L complexes using HRMS}

Before initial validation and dual detection protocols, the M-L complexes first had to be confirmed and readily reproducible under concentrated acidic digestion conditions. First, ICP standards were mixed with the complexing agents and were monitored via the QQQ using the Q1 scan mode. Preliminary observations demonstrated unique signals in the mass spectrum showing similar isotopic patterns that coincided with the investigated metals. A more in-depth investigation revealed these metals formed nitrated adducts since the ICP standards possessed $2 \%$ nitric acid within the solution. Additionally, other mass spectrum structures showed various complexes with similar distributions related to water and sodium adducts.

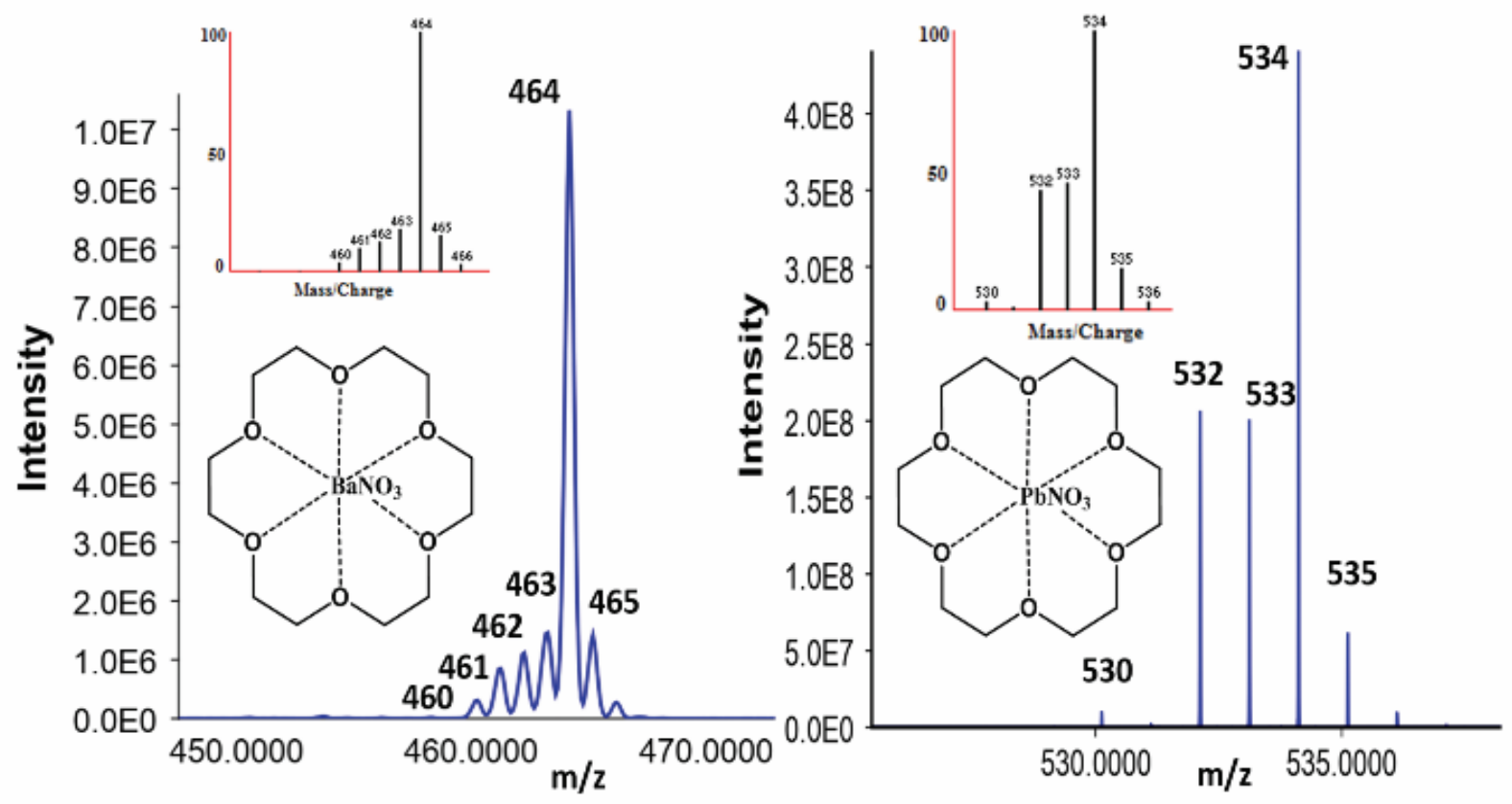

Figure 4.3: Orbitrap confirmation of $18 \mathrm{C} 6\left[\mathrm{Ba}-\mathrm{NO}_{3}\right]$ with $\mathrm{Ba}$ natural abundance (left) and $[\mathrm{Pb}$ $\mathrm{NO}_{3}$ ] with $\mathrm{Pb}$ natural abundance (right) patterns correlating to the simulated $\mathrm{M}-\mathrm{L}$ isotopic distribution. 
A Q-Exactive ${ }^{\circledR}$ orbitrap was utilized to corroborate the findings and confirm the M-L complexes' identity from the QQQ full scan. Flow injection analysis (FIA) and manual manipulations of the source conditions revealed the presence of $1: 1$ as well as 1:2 ratios metal to ligand complexes. Additionally, water adducts could also be observed but were less abundant than the nitrated species. A full fragmentation breakdown and isotopic ratio observation are illustrated in Figure 4.3 and Table 4.1 for barium and lead species. Unfortunately, an Sb complex could not be formed with the chosen macrocycle; however, a different mechanism and complexing agent unlocked the identification and will be discussed later.

Table 4.1: Orbitrap confirmation of fragmentation patterns of nitrated and lone metal species.

\begin{tabular}{|c|c|c|c|c|}
\hline Iden & Ions $(\mathrm{m} / \mathrm{z})$ observed & Identification & $M S^{I}$ (nitrated forms) & $M S^{n}$ (lone metals) \\
\hline \multirow{5}{*}{$\begin{array}{c}\text { Barium } \\
\mathrm{BaNO}_{3}(200 \mathrm{Da}) \\
18 \mathrm{C6}(264 \mathrm{Da}) \\
\mathrm{C}_{12} \mathrm{H}_{24} \mathrm{O}_{6}\end{array}$} & 464.0498 & {$\left[\mathrm{~L}+{ }^{138} \mathrm{Ba}+\mathrm{NO}_{3}\right]^{+}$} & $200.9003\left[{ }^{138} \mathbf{B a}+\mathrm{NO}_{3}\right]^{+}$ & $137.9003\left[{ }^{\mathbf{1 3 8}} \mathbf{B a}\right]^{+}$ \\
\hline & 463.0504 & {$\left[\mathrm{~L}+{ }^{137} \mathrm{Ba}+\mathrm{NO}_{3}\right]^{+}$} & $199.9009\left[{ }^{137} \mathbf{B a}+\mathrm{NO}_{3}\right]^{+}$ & $136.9009\left[{ }^{137} \mathbf{B a}\right]^{+}$ \\
\hline & 462.0491 & {$\left[\mathrm{~L}+{ }^{136} \mathrm{Ba}+\mathrm{NO}_{3}\right]^{+}$} & $198.8997\left[{ }^{\mathbf{1 3 6}} \mathbf{B a}+\mathrm{NO}_{3}\right]^{+}$ & $135.8997\left[{ }^{\mathbf{1 3 6}} \mathbf{B a}\right]^{+}$ \\
\hline & 461.0502 & {$\left[\mathrm{~L}+{ }^{135} \mathrm{Ba}+\mathrm{NO}_{3}\right]^{+}$} & $197.9008\left[{ }^{\mathbf{1 3 5}} \mathrm{Ba}+\mathrm{NO}_{3}\right]^{+}$ & $134.9008\left[{ }^{135} \mathbf{B a}\right]^{+}$ \\
\hline & 460.0491 & {$\left[\mathrm{~L}+{ }^{134} \mathrm{Ba}+\mathrm{NO}_{3}\right]^{+}$} & $196.8972\left[{ }^{\mathbf{1 3 4}} \mathbf{B a}+\mathrm{NO}_{3}\right]^{+}$ & $133.8972\left[{ }^{134} \mathrm{Ba}^{+}\right.$ \\
\hline \multirow{4}{*}{$\begin{array}{c}\text { Lead } \\
\mathrm{PbNO}_{3}(270 \mathrm{Da}) \\
18 \mathrm{C6}(264 \mathrm{Da}) \\
\mathrm{C}_{12} \mathrm{H}_{24} \mathrm{O}_{6}\end{array}$} & 534.0498 & {$\left[\mathrm{~L}+{ }^{208} \mathrm{~Pb}+\mathrm{NO}_{3}\right]^{+}$} & $270.1003\left[{ }^{208} \mathbf{P b}+\mathrm{NO}_{3}\right]^{+}$ & $208.1003\left[{ }^{208} \mathbf{P b}\right]^{+}$ \\
\hline & 533.0504 & {$\left[\mathrm{~L}+{ }^{207} \mathrm{~Pb}+\mathrm{NO}_{3}\right]^{+}$} & $269.0109\left[{ }^{207} \mathbf{P b}+\mathrm{NO}_{3}\right]^{+}$ & $207.0109\left[{ }^{207} \mathbf{P b}\right]^{+}$ \\
\hline & 532.0491 & {$\left[\mathrm{~L}+{ }^{206} \mathrm{~Pb}+\mathrm{NO}_{3}\right]^{+}$} & $268.0097\left[{ }^{206} \mathbf{P b}+\mathrm{NO}_{3}\right]^{+}$ & $206.0097\left[^{206} \mathbf{P b}\right]^{+}$ \\
\hline & 530.0491 & {$\left[\mathrm{~L}+{ }^{204} \mathrm{~Pb}+\mathrm{NO}_{3}\right]^{+}$} & $266.0012\left[{ }^{204} \mathbf{P b}+\mathrm{NO}_{3}\right]^{+}$ & $204.0012\left[\left[^{204} \mathbf{P b}\right]^{+}\right.$ \\
\hline
\end{tabular}

After orbitrap verification, source conditions and optimizations were performed on the QQQ using the Optimizer® software. Fragmentor and collision energy voltages were monitored from $10-100 \mathrm{~V}$ and $10-250 \mathrm{~V}$ with $10 \mathrm{~V}$ increments to yield the highest signal possible, respectively. The most abundant product ions for the barium-complex were the nitrated species (200.1 Da) and the lone metal species (208.1 Da) for the lead complex. Because of the mechanism and factors for complex formation, the decreasing $\mathrm{m} / \mathrm{z}$ values must be included when building the acquisition method. For instance, the barium-complex forms various precursor ion $m / z$ values consistent with 
its isotope values at 464.1 (138), 463.1 (137), 462.1 (136), 461.1 (135), and 460.1 (134). Therefore, the acquisition method must account for these values to gain confidence in the observed structure, which is not required for OGSR analysis.

\section{Choices of complexing agents}

Our goal was to select a single complexing agent to capture the three critical elemental constituents of GSR (barium, lead, and antimony). However, extensive literature review and experimentation demonstrated that this was not possible. Antimony (a metalloid like arsenic and bismuth) demonstrated different complexation characteristics that necessitated selection of a separate chelating agent for this element. While this adds another ingredient to the complexing solution, we demonstrated that it is still possible to detect $\mathrm{Ba}, \mathrm{Pb}$, and $\mathrm{Sb}$ via $\mathrm{LC}-\mathrm{MS} / \mathrm{MS}$.

\subsubsection{Crown ethers}

Interest in supramolecular chemistry has expanded from its early conceptions by van der Waals into Pedersen's initial discovery of simple macrocycles. These compounds are integrated into various disciplines such as nanotechnology, medicinal chemistry, chemo-sensors, and others [56]. Depending on the guests and applications, modifications such as heteroatom substitutions or functional group additions provide scientists various avenues for analyte characterization. Because of their versatility to encompass a myriad of guest molecules easily, crown ethers are one of the most utilized and studied macrocycles in the field via self-assembly.

Crown ethers employ various binding mechanisms, including electrostatic interactions, hydrogen bonding, and/or van der Waals forces, depending on the guest analyte. Like other host compounds, these molecules have shown their effectiveness in various fields, including forensics for explosive and gunshot residue analysis to toxic metal removal in wastewater $[35,57,58]$. The strength of complexation is dependent on various factors, including the internal cavity size of the 
crown ether, the atomic radii, and the charge of analytes. The appealing aspect of these interactions involves identifying metal ions and their robust nature in forming metal-ligand complexes. The creation of these complexes increases an analyte's molecular weight while maintaining natural isotopic abundances. Additionally, the host macrocycles provide transportation and interactions through a column for MS/MS detection.

Characterizing complexes relies heavily on the instrumentation parameters. Previous experiments performed in our group focused on mobilities via ion mobility spectrometry CID experimentation via LC-MS/MS [35,59]. For instance, when inspecting Pb, the addition of $18 \mathrm{C} 6$ increases the molecular weight by 264.1 Da yielding 472.1 Da for the lone metal and 534.1 Da when nitrated. When the 534.1 Da complex is fragmented, the $\mathrm{Pb}-\mathrm{NO}_{3}$ (270 Da) product ion is predominant and displays similar isotopic distributions to the lead ion. When the $270 \mathrm{Da}$ is fragmented further, the $208.1(52 \%), 207.1(22 \%), 206.1(24 \%)$, and $204.1(2 \%)$ ions are observed, thus solidifying high confidence for the precursor complex in question.

Other aspects like charge state and size of ions are essential when forming complexes. Additionally, competition for the macrocycle's internal cavity can ultimately affect the response of the metal detection. Because of validation protocols, internal standards should be similar in structure and exhibit comparable ionization energies to measure analyte concentrations accurately. For the OGSR method, $\mathrm{D}_{10}$-diphenylamine was coeluted with DPA but possessed unique transitions for simple identification. The IGSR method used thallium (Tl) as the internal standard since it is typically not found in GSR and is similar in size $(150 \mathrm{pm})$ to both $\mathrm{Ba}^{2+}(135 \mathrm{pm})$ and $\mathrm{Pb}^{2+}(119 \mathrm{pm})$. Without $\mathrm{Tl}^{+}$present, $18 \mathrm{C} 6$ showed preferential binding for $\mathrm{Ba}$ versus $\mathrm{Pb}$, illustrated in Figure S4.1. Thallium lowered the intensities for IGSR constituents yielding the selectivity trend of $\mathrm{Tl}>\mathrm{Ba}>\mathrm{Pb}$. In contrast, antimony $(76 \mathrm{pm})$ showed no efficient binding affinity with 
18C6. This observation shows that the size and charge of the guest play a crucial role in binding selectivity.

\subsubsection{Tartaric acid}

In our studies, however, antimony ( $\mathrm{Sb}$ ) has not been observed to self-assemble with various crown ether species, including 12-crown-4-ether, 15-crown-5-ether, 18-crown-6-ether, and dibenzo-18-crown-6-ether. Various research groups have explored and observed tartaric acid's effectiveness in binding with antimony through a process known as chelation [60-62]. Chelation occurs by forming two or more coordinate bonds between a polydentate ligand (tartaric acid) and a central atom (metal cations). This ligand is naturally found in fruits and is utilized in ceramics and pharmaceuticals. Several groups have investigated the interaction between various metal cations and tartaric acid structures under negative ionization modes [62-64].

Additionally, tartaric acid can yield enantiomeric complexes (D- and L-) and be separated by HPLC and capillary electrophoresis [65]. For adequate separations, the tartrate complexes can be dissolved in water and salt solvents and further yet, diluted in various working stock solutions with specific solvents such as methanol. Tartaric acid is readily dissolvable in water but not in methanol, which is why more water adducts are observed versus other salts. 


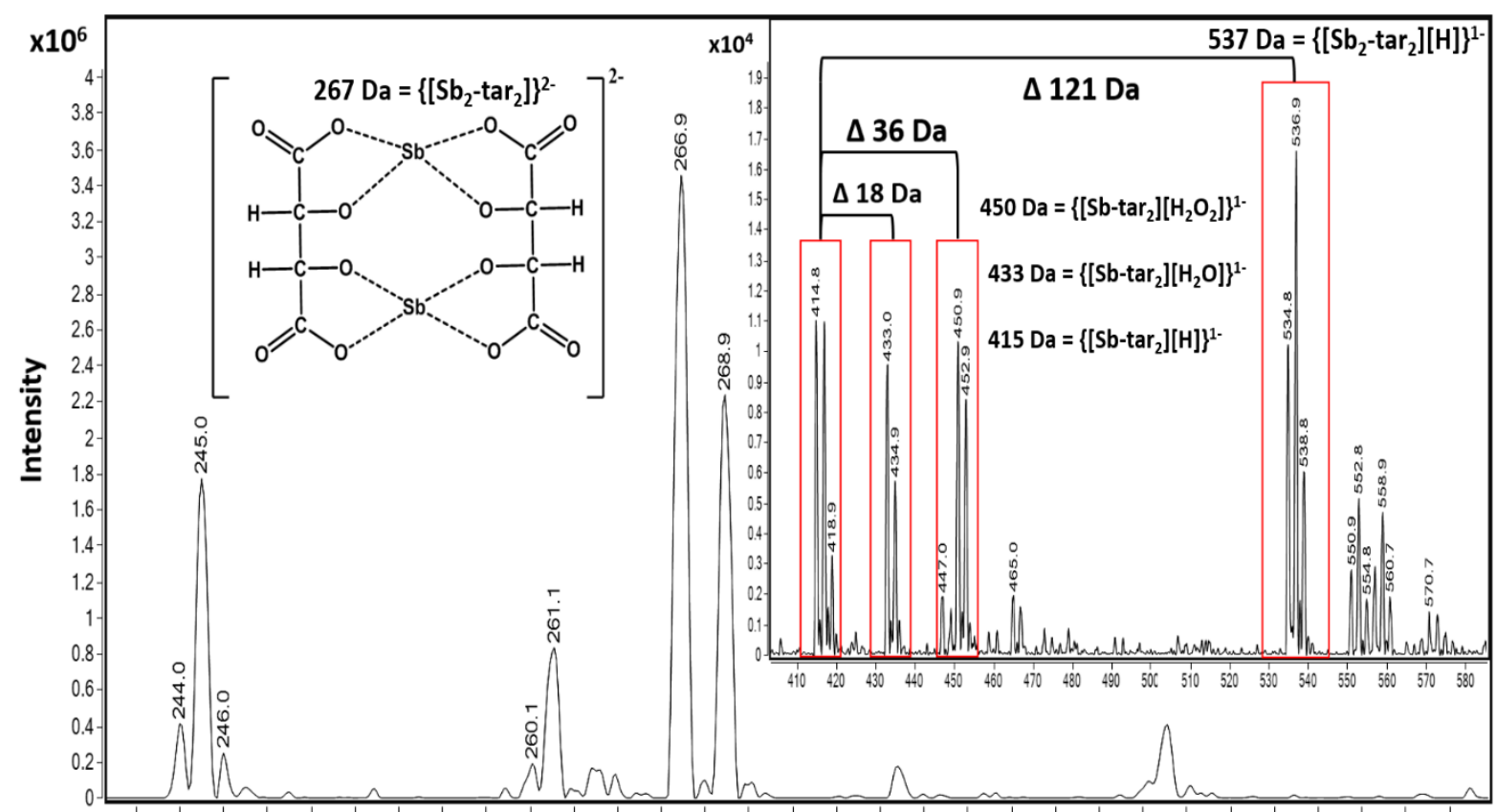

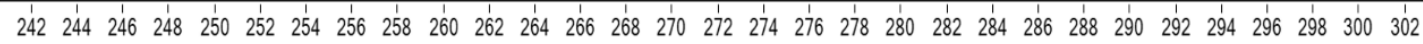

Counts vs. Mass-to-Charge $(\mathrm{m} / \mathrm{z})$

Figure 4.4: Flow injection analysis of the various tartrate complexes with $\mathrm{Sb}$ in ESI- mode. Adducts formation is still prevalent, and because of the nature of the ligand, multiple Sb ions can bind to multiple ligands. For instance, the difference between $415 \mathrm{Da}$ and $433 \mathrm{Da}$ is an $\mathrm{m} / \mathrm{z}$ value of 18, a water molecule. However, from $415 \mathrm{Da}$ to $537 \mathrm{Da}$ is the addition of an $\mathrm{Sb}$ ion.

However, the adducts may also be attributed to altering voltages of an ESI source. This can cause the isotope ratios to differ from recorded natural abundances $57 \%\left({ }^{121} \mathrm{Sb}\right)$ and $43 \%\left({ }^{123} \mathrm{Sb}\right)$, outlined by Schug et al. and other groups (Figure 4.4) [61,62,66-69]. For instance, by altering the nozzle voltage from $500 \mathrm{~V}$ to $2500 \mathrm{~V}$, the complex at $537 \mathrm{~m} / \mathrm{z}$ appears and disappears, respectively. This investigation showed that the structure of the tartrate complexes can shift in not only the adducts but also in the charge states. This finding has been outlined by Schug and others by altering the voltages of both the fragmentor and within the collision chamber $[62,64]$. The tartrate complexes can undergo homolytic cleavages as the complex reorients throughout the mass analyzer. Thus, resulting in the different losses of peroxides and water adducts. From these observations, the ligand tends to favor the complex with 2- charge (267 Da) than the 1- charged complexes $(415,433,450$, and 537 Da) illustrated in Figure 4.4. 
Prior knowledge of complexation illustrated that 18C6 was not as sensitive to $\mathrm{Sb}$ versus $\mathrm{Ba}$ and $\mathrm{Pb}$; therefore, tartaric acid was tested as the possible "one host" solution. Although both $\mathrm{Ba}$ and $\mathrm{Pb}$ formed complexes with the tartaric acid, their signals were vastly smaller than $\mathrm{Sb}$. Ultimately, we decided to incorporate both complexing agents, tartaric acid and 18C6, in equal parts for the final solution.

\section{Analytical validation and figures of merit}

\subsubsection{Analytical validation and figures of merit}

Tables 4.2 and 4.3 represent both OGSR and IGSR constituents' figures of merit, including the LOD, LOQ, \%RSD (intra- and inter-day), and linear dynamic range (LDR). The OGSR compounds LDR expanded from 1-200 ppb (1-200 pg), and the 1:1 IGSR complexes $100 \mathrm{ppb}$ to $25 \mathrm{ppm}$ (1.0-250 ng). The mean, standard deviation, and 95\% confidence intervals are measured against the $\mathrm{D}_{10}$-DPA and Tl quantifier ion ratios for the OGSR and IGSR, respectively. The Agilent MassHunter® Quantitative Analysis software was used to calculate the bias, LDR, and other figures of merit. The absolute LOD and LOQ (ppb and ppm) were calculated for a $1 \mu \mathrm{L}$ injection and $10 \mu \mathrm{L}$ injection for OGSR and IGSR, respectively. The estimated LOD and LOQ (pg and ng) were calculated and adjusted for the injections previously mentioned.

Table 4.2: Figures of merit of organic compounds. Included is information pertaining to LOD, LOQ, LDR, the precursor ions, and the ion ratio of the most abundant qualifier ion.

\begin{tabular}{|c|c|c|c|c|c|c|c|c|c|c|c|}
\hline Group & Constituent & LOD (ppb) & LOD (pg) & LOQ (ppb) & LOQ (pg) & $\begin{array}{l}m / z \text { MRM }[\mathrm{M}+\mathrm{H}]^{+} \\
\text {Precursor: Product }\end{array}$ & $\begin{array}{c}\text { Ion ratio\% } \\
(\mathbf{n}=15)\end{array}$ & $\mathbf{R}^{2}$ & $\begin{array}{l}\text { \%RSD } \\
\text { INTRA }\end{array}$ & $\begin{array}{l}\text { \% RSD } \\
\text { INTER }\end{array}$ & LDR (pg) \\
\hline \multirow{7}{*}{ مُ } & AK 2 & 0.3 & 0.3 & 0.90 & 0.90 & 227:170:93 & 70.7 & 0.999 & 1.3 & 4.8 & $1-200$ \\
\hline & DPA & 3.4 & 3.4 & 10.1 & 10.1 & 170:93:65 & 20.4 & 0.998 & 3.7 & 10.4 & $10-200$ \\
\hline & EC & 1.0 & 1.0 & 3.0 & 3.0 & 269:148:120 & 91.6 & 0.999 & 1.1 & 9.3 & $5-200$ \\
\hline & MC & 0.30 & 0.30 & 0.90 & 0.90 & 241:134:106 & 56.0 & 0.999 & 2.7 & 11.0 & $1-200$ \\
\hline & N-NDPA & 4.6 & 4.6 & 13.9 & 13.9 & 199:169:65 & 12.7 & 0.997 & 4.8 & 6.0 & $25-200$ \\
\hline & 4-NDPA & 3.0 & 3.0 & 9.0 & 9.0 & 215:198:167 & 92.0 & 0.999 & 7.9 & 6.4 & $10-200$ \\
\hline & 2-NDPA & 2.7 & 2.7 & 8.2 & 8.2 & 215: 180 & 100.0 & 0.997 & 4.6 & 4.0 & $10-200$ \\
\hline
\end{tabular}


Table 4.3: Figures of merit for inorganic compounds. Included is information pertaining to LOD, LOQ, LDR, the precursor ions, and the ion ratio of the most abundant qualifier ion.

\begin{tabular}{|c|c|c|c|c|c|c|c|c|c|c|c|}
\hline Group & Constituent & LOD (ppm) & LOD (ng) & LOQ (ppm) & LOQ (ng) & $\begin{array}{c}m / z \text { MRM } \\
\text { Precursor: Product }\end{array}$ & $\begin{array}{l}\text { Ion ratio } \\
\%(n=15)\end{array}$ & $\mathbf{R}^{2}$ & $\begin{array}{l}\text { \%RSD } \\
\text { INTRA }\end{array}$ & $\begin{array}{l}\text { \%RSD } \\
\text { INTER }\end{array}$ & LDR (ng) \\
\hline \multirow{3}{*}{ 范 } & $\mathbf{B a}$ & 0.10 & 1 & 0.30 & 3.0 & $\begin{array}{l}\mathbf{4 6 4}: 200: 138 \\
\mathbf{4 6 3}: 199: 137 \\
\mathbf{4 6 2}: 198: 136 \\
\mathbf{4 6 1}: 197: 135\end{array}$ & $\begin{array}{c}71.70(138) \\
11.23(137) \\
7.85(136) \\
6.59(135)\end{array}$ & 0.998 & 8.4 & 4.6 & $1-60$ \\
\hline & $\mathbf{P b}$ & 0.20 & 2 & 0.60 & 6.0 & $\begin{array}{l}\text { 534:270:208 } \\
\text { 533:269:207 } \\
\text { 532:268:206 }\end{array}$ & $\begin{array}{l}52.40(208) \\
22.10(207) \\
24.10(206)\end{array}$ & 0.982 & 6.4 & 5.7 & $2-60$ \\
\hline & Sb & 5.0 & 50 & 15.0 & 150 & $\begin{array}{l}\text { 267:121 } \\
\text { 269:123 }\end{array}$ & $\begin{array}{l}57.21(121) \\
42.79(123)\end{array}$ & 0.974 & 2.7 & 11.0 & $50-250$ \\
\hline
\end{tabular}

As mentioned before, the LOD and LOQ values were improved from the previous proofof-concept study for both IGSR and OGSR. For OGSR, we included an additional qualifier ion barring 2-NDPA, while Ali et al. served as a guide for source parameters due to similar instrumentation conditions [33]. Improvement of the IGSR constituents was contributed to manual optimization and understanding of the complexation process. If more agent is present, more guest analyte can be encapsulated for lower detection. The LDR for $\mathrm{Sb}$ is higher due to its formation nature and a greater ionization potential and energies required to dissociate the complex. It is important to note that the 50-250 $\mathrm{ng}$ range for $\mathrm{Sb}$ is on the cusp of the range when referencing authentic samples. Therefore, we continue to monitor its presence but will need further investigations to circumvent the complex's ionization energy.

The linearity of each compound was assessed through calibration curves and intra- and interday studies. All compounds and elements showed a linear response with an $R^{2}$ value $\geq 0.974$. The working calibration ranges were $1 \mathrm{ppb}$ to $200 \mathrm{ppb}$ (OGSR) and $100 \mathrm{ppb}$ to $25 \mathrm{ppm}$ (IGSR). Further statistical analysis evaluated an accurate quantitation, including $R^{2}$ coefficients and residual plots with less than $10 \%$ RSD. Residuals plots assess the nature of the samples collected using several considerations: 1) randomly distributed, 2) the variances are equal, and 3) values are normally distributed across predicted values. The residuals' variance did not increase with 
concentration until after $200 \mathrm{ppb}$ and $6 \mathrm{ppm}(\mathrm{Ba}$ and $\mathrm{Pb})$ and $25 \mathrm{ppm}(\mathrm{Sb})$. This observation confirms homoscedasticity across data points, thus ensuring randomness. The relationship between the independent and dependent variables and their variances is displayed in Figure S4.2.

\subsubsection{Background and collection substrate considerations: recovery study and comparison to ICP-MS digestion method}

A critical aspect of the method validation was to consider the performance and practicality of the substrate used for the collection and retention of the inorganic and organic components of interest from the hands of an individual. For this purpose, we compared the recovery of IGSR and OGSR from tesa® Tack polymer and the more universal sampling media carbon adhesive. Our goal was to evaluate if the LC-MS/MS method can incorporate the use of carbon stubs to be more compatible with current practice and avoid the change of sampling protocols widely used in the GSR community by both law enforcement and laboratory personnel.

The surface extraction of each substrate was evaluated for any possible suppressants. In part, this concern was due to the substrates possessing both polymeric and adhesive qualities that could potentially interfere with the column performance. We were less concerned with the selectivity against the substrates due to choosing specific $m / z$ values and MRM transitions unique to elements and compounds.

Our previous study successfully used tesa® Tack polymer for GSR collection by fully submerging and exposing it to organic solvents and harsh acidic conditions [35]. This polymer was very attractive in our initial study because it was commercially sold (http://www.tesa.com) as a non-greasy, putty and suggested by BKA colleagues based on their experiences (Dr. Ludwieg Niewoehner) and adapted for the previous project [35]. However, because we want this method to comply with GSR cases, without changing current sampling protocols, the universal method of 
carbon tape stubs was evaluated. Thus, a newly developed extraction method was optimized for both OGSR and IGSR components for the carbon tape by utilizing surface washings. These washings displayed suppression of analytical signals, which led to the incorporation of filters. The OGSR extraction used $100 \%$ methanol, where the IGSR analysis used a micro-bulk method that needed heat and concentrated acids as microliter volumes.

One substantial difference between this work and our previous proof-of-concept study is the OGSR and IGSR recovery assessment from the substrates. Following the Eurachem validation guideline, we conducted a recovery comparison experiment using ESI involving both carbon tape and tesa ${ }^{\circledR}$ Tack [35]. Bias or percent recovery is not only crucial to indicate potential trends or underlying characteristics/properties present but addresses the effectiveness of a method by measuring the "closeness" of extraction results versus the reference or "true" spiked value onto substrates.

There are two critical differences between the tesa® Tack and carbon tape. First, the polymer exhibits porous properties similar to the skin's epidermal layer, allowing for significant absorption of OGSR. Secondly, the thickness is vastly different compared to the carbon tape. Since tesa® Tack is thicker than the carbon tape, the OGSR components can adsorb into the tack's inner layers, creating a more significant challenge for extraction. Three different concentration levels (low, medium, high) assessed the repeatability and reproducibility and determined the variability in the results; $25 \mathrm{ppb}, 50 \mathrm{ppb}$, and $165 \mathrm{ppb}$ for OGSR (Table 4.4) and 1 ppm, 3 ppm, and 5 ppm for IGSR (Table 4.5). The low, medium, and high values were chosen to represent concentrations that can be fully quantified from the validated method and represent concentrations observed in authentic items. 
Table 4.4: Summary of OGSR analyte recoveries on both carbon tape and tesa® Tack substrate.

\begin{tabular}{c|c|cccccc}
\hline $\begin{array}{c}\text { Expected } \\
\text { Conc. }\end{array}$ & Substrate & $\begin{array}{c}\text { MC } \\
\text { \% Recovery }\end{array}$ & $\begin{array}{c}\text { EC } \\
\text { \% Recovery }\end{array}$ & $\begin{array}{c}\text { DPA } \\
\text { \% Recovery }\end{array}$ & $\begin{array}{c}\text { N-NDPA } \\
\text { \% Recovery }\end{array}$ & $\begin{array}{c}\text { 4-NDPA } \\
\text { \% Recovery }\end{array}$ & $\begin{array}{c}\text { 2-NDPA } \\
\text { \%ecovery }\end{array}$ \\
\hline \multirow{2}{*}{$\mathbf{2 5}$ ppb } & Carbon Tape & $\mathbf{4 1 . 2} \pm 0.74$ & $\mathbf{4 2 . 1} \pm 2.81$ & $\mathbf{5 9 . 9} \pm 3.29$ & $\mathbf{8 5 . 0} \pm 2.34$ & $\mathbf{4 4 . 8} \pm 5.87$ & $\mathbf{8 3 . 4} \pm 5.33$ \\
& tesa® Tack & $\mathbf{2 8 . 8} \pm 1.49$ & $\mathbf{1 7 . 3} \pm 1.59$ & $\mathbf{3 6 . 9} \pm 1.69$ & $\mathbf{5 5 . 2} \pm 3.83$ & $\mathbf{1 7 . 8} \pm 8.45$ & $\mathbf{5 2 . 9} \pm 11.2$ \\
\hline \multirow{2}{*}{$\mathbf{5 0}$ ppb } & Carbon Tape & $\mathbf{7 7 . 5} \pm 1.47$ & $\mathbf{6 7 . 8} \pm 1.36$ & $\mathbf{7 4 . 2} \pm 1.71$ & $\mathbf{8 3 . 5} \pm 2.43$ & $\mathbf{4 0 . 4} \pm 1.38$ & $\mathbf{8 6 . 2} \pm 1.60$ \\
& tesa ${ }^{\circledR}$ Tack & $\mathbf{3 8 . 6} \pm 0.40$ & $\mathbf{2 7 . 1} \pm 0.95$ & $\mathbf{5 5 . 6} \pm 0.51$ & $\mathbf{4 8 . 5} \pm 9.03$ & $\mathbf{2 3 . 4} \pm 1.15$ & $\mathbf{5 7 . 7} \pm 1.49$ \\
\hline \multirow{2}{*}{$\mathbf{1 6 5}$ ppb } & Carbon Tape & $\mathbf{6 1 . 7} \pm 2.59$ & $\mathbf{8 0 . 5} \pm 1.58$ & $\mathbf{5 7 . 6} \pm 1.25$ & $\mathbf{5 4 . 9} \pm 1.77$ & $\mathbf{3 5 . 7} \pm 0.77$ & $\mathbf{5 3 . 5} \pm 1.00$ \\
& tesa® Tack & $\mathbf{3 0 . 5} \pm 2.52$ & $\mathbf{2 7 . 1} \pm 1.86$ & $\mathbf{3 5 . 5} \pm 1.24$ & $\mathbf{2 4 . 7} \pm 1.70$ & $\mathbf{2 0 . 6} \pm 1.29$ & $\mathbf{2 3 . 5} \pm 0.78$ \\
\hline
\end{tabular}

From the recovery experiment, the OGSR constituents' results indicated that all components had less than $10 \% \%$ RSD, barring 2-NDPA at 25 ppb for the tesa ${ }^{\circledR}$ Tack, $11.2 \%$. The percent recovery values ranged from $35.7-86.2 \%$ for carbon tape and $17.3-57.7 \%$ for tesa® Tack. A one-tailed t-test (at $p=0.05$ ) showed the carbon tape has significantly higher recoveries than the tesa® Tack at all concentration ranges tested.

It is worth mentioning the extraction methods are not exhaustive as we designed the method for surface washing rather than a complete submersion extraction. The rationale for this decision was two-fold: 1) it minimizes potential undesired contribution from the adhesive that can be detrimental for LC-MS, and 2) gentle to mild washings further increase the possibility of not displacing particles for further analysis, as demonstrated by Bonnar et al. [11].

Conversely, the IGSR percent recoveries were comparable for both substrates, showing no significant differences between $\mathrm{Ba}$ and $\mathrm{Pb}$ observed using a one-tail t-test. The recovery range for the carbon tape was $44-85 \%$, and the tesa ${ }^{\circledR}$ Tack ranged from $43-86 \%$, which is illustrated by Table 4.5. As a result, the carbon tape was chosen for the remaining of the study as it improved OGSR recovery while not having a detriment effect in the IGSR, as compared to the tesa® Tack. 
Table 4.5: Summary of IGSR analyte recoveries using ICP-MS standards on both substrates.

\begin{tabular}{c|c|cc}
\hline Expected Conc. & Substrate & Ba \% Recovery & Pb \% Recovery \\
\hline \multirow{2}{*}{1 ppm } & Carbon Tape & $\mathbf{6 7 . 1} \pm 0.97$ & $\mathbf{5 7 . 8} \pm 3.28$ \\
& tesa® Tack & $\mathbf{5 7 . 9} \pm 1.67$ & $\mathbf{4 5 . 5} \pm 2.98$ \\
\hline \multirow{2}{*}{$3 \mathbf{~ p p m}$} & Carbon Tape & $\mathbf{7 4 . 7} \pm 2.44$ & $\mathbf{4 4 . 4} \pm 3.13$ \\
& tesa® Tack & $\mathbf{8 3 . 3} \pm 4.53$ & $\mathbf{4 4 . 6} \pm 1.39$ \\
\hline \multirow{2}{*}{$5 \mathbf{~ p p m}$} & Carbon Tape & $\mathbf{8 4 . 7} \pm 3.12$ & $\mathbf{4 4 . 3} \pm 1.27$ \\
& tesa® Tack & $\mathbf{8 6 . 1} \pm 1.14$ & $\mathbf{4 3 . 5} \pm 1.05$ \\
\hline
\end{tabular}

This procedure utilized ICP-MS standards directly spiked onto each substrate and allowed to dry completely. After drying, surface extractions followed, which used concentrated acids and the micro-bulk procedure mentioned previously. However, this only answers one part of the recovery process as those standards do not truly represent a particulates' nature because they are already solubilized. Therefore, a standard that corresponds to the morphological characteristics and composition relating to ASTM E1588-20 would better represent authentic samples and monitor the behavior of IGSR in concentrated acid washings and digestion, as discussed below.

\subsubsection{Cross-validation of recovery of IGSR via ICP-MS}

With OGSR compounds, variables like lipophilicity and evaporation rates play a significant role in the recovery from exposed skin and other sampling areas. Because the environment heavily influences IGSR formation and placement, sufficient analysis is more dependent on factors like extraction procedures and substrate surfaces versus skin absorption. Additionally, OGSR standards correlate more with authentic samples than ICP-MS standards spiked onto substrate surfaces. Therefore, intact IGSR particulates offer a greater evaluation of factors like extraction protocols and interactions.

Tailor-made primer-only GSR ( $p$-GSR) standards originating from a Winchester primer was previously developed and characterized in our group and was used to evaluate the extraction protocol's effectiveness [45]. These standards are made of $p$-GSR obtained from the actual 
discharge of a primer in a firearm, therefore providing similar conditions and concentrations that an analyst would expect from an authentic sample. These "matrix-matched" microparticle standards were characterized via ICP-MS, SEM/EDS, and LIBS methods to confirm the concentration, stability, and elemental composition and morphology of the particles. Results showed that this standard correlates to the expected concentration found in the primers [45].

Since IGSR is more susceptible to secondary and tertiary transfer, factors like substrate composition and extraction protocol can affect practitioners' analysis. We address both by substrate exposure to heavy acids with multiple washings to a final volume of $500 \mu \mathrm{L}$. Therefore, both the extraction and substrate considerations were compared via digestion methods; $85^{\circ}$ for 10 minutes in concentrated acid (LC-MS/MS) versus 60 minutes with dilution to $10 \%$ nitric acid (ICP-MS). Our findings demonstrated that the percent recoveries were higher with the 60-minute digestion protocol but with less than a $10 \%$ difference. Furthermore, there was no statistically significant difference between tesa® Tack and carbon tape regarding the recovery of $\mathrm{Pb}$ and $\mathrm{Ba}$. This difference explains more of the interaction with the 18-crown-6-ether, where the binding energy is dependent on the size and charge of the metallic species present in a solution.

\subsubsection{Workflow and cross-validation with screening methods}

Within the forensics community, there is an increased interest to incorporate rapid, preliminary techniques for IGSR and OGSR, followed by confirmatory analyses [13,24,34]. By introducing these methodologies, analysts can filter presumptively "negative" results and focus on confirmation of those positive items, ultimately making better-informed decisions and reducing backlogs. With this increased interest, we tested the applicability of implementing multiple screening techniques followed by the proposed LC-MS/MS methodology from a single firing event and sample. 
After the collection via carbon-adhesive stub, LIBS was used as the first instrument for analysis. LIBS has several appealing aspects, including ambient conditions, quick analysis times ( $<2$ minutes/stub), and compositional information comparable to SEM/EDS [47]. Because of the ambient and laser conditions, the OGSR constituents are not substantially lost, and the overall IGSR morphology remains practically uncompromised. The preservation of both constituents allows for further analysis, such as electrochemical methods. By washing a portion of the sample's surface with two solvents (acetonitrile and aqueous buffer), OGSR and IGSR are effectively extracted. With an analysis time comparable to LIBS (< 5-10 minutes), the advantage of electrochemical methods is the capability to simultaneously detecting both constituents [46]. Furthermore, combining these screening methods has demonstrated their effectiveness in increasing confidence in GSR samples with accuracies superior to 95\%, with the advantage that analysis can be followed by SEM/EDS confirmation [12]. Because electrochemical methods generate extracts compatible with LC-MS/MS, this study explored if these washings could be used with the micro-bulk digestion and complexing agents.

Because LC-MS/MS offers superior LOD and LOQ and consumes a small sample volume $(1 \mu \mathrm{L})$, the remaining aliquot can be used for further confirmation methodologies, if needed. After running the organic extract from electrochemistry, the aqueous buffer aliquot with the organic extract can be combined and pushed through with the micro-bulk digestion and complexing agent addition allowing OGSR and IGSR identification by LC-MS/MS. Additionally, we tested the electrochemical extraction collection efficiency by running subsequent washings from an extracted stub. Our findings showed that the subsequent washings show either no signal or signal below the LOQ, which confirms that the electrochemical extractions are very efficient in collecting both 
constituents. These results illustrate the potential of utilizing various instrumentation techniques to gain more informative data from a single sample without compromising the entire stub.

\subsubsection{Performance rates and criteria for authentic samples}

Since dual detection of IGSR and OGSR is not routinely conducted at forensic laboratories, a criterion outlining the requirements for identifying "true positive" and "true negative" results must be established. The lack of guidelines for OGSR compounds versus the well-established ASTM protocol for IGSR is the primary contributing factor. Therefore, we highlight the considerations to call a sample positive for GSR when: 1) evidence of both OGSR compounds and IGSR elements must be present above LOD and background population-based critical thresholds, 2) a minimum of three components, either two OGSR components, and one IGSR analyte or vice versa. For example, if the sample contains EC, DPA, and Ba, the sample is labeled "positive for GSR." If the sample only contains one of the GSR components, then the sample is labeled "potential GSR" and needs further analysis. For example, if only OGSR compounds such as EC, DPA, and 2-NDPA are present without the detection of $\mathrm{Pb}, \mathrm{Ba}$, or $\mathrm{Sb}$, that sample is characterized as "potential GSR". In the ASTM E1588-20, "potential GSR" is referred to as consistent with or commonly associated with GSR; however, due to the lack of consensus at this moment concerning the confirmatory value of OGSR/IGSR profiles, we followed a more conservative two-category scale for positive results (characteristic or positive, and potential). Our experience with the interpretation of large population sets with LIBS and EC demonstrates that machine learning algorithms outperform the categorical critical threshold approach used here, with the added advantage of providing a probabilistic output for a more objective interpretation of the evidence. We are currently collecting a more extensive population to apply this probabilistic approach to LC-MS/MS data. Larger sample sizes are needed to split the data into training and 
testing/validation sets with enough statistical power. Nonetheless, the critical threshold method is valuable as an exploratory tool to learn about the distribution of the data in different subpopulations and provide a basis for interpreting computerized approaches.

We tested this criterion against two types of authentic sample data sets and compare them with LIBS and electrochemical methods. The two data sets consisted of 95 baseline shooter samples (Table 4.6) and 78 activity samples (Table 4.7). The baseline samples involved shooting in the WVU ballistics range and immediately collecting after a shooting event. These samples were extracted using the LC-MS/MS methodology involving the filters and concentrated acid surface washings. The baseline results are represented in Table 4.6 and include success rates and detection for LC-MS/MS. The collection methodology involves generating four separate carbon stub samples labelled as such, left-palm (LP), left-back (LB), right-palm (RP), and right-back (RB). The first shooting session used a single type of ammunition (Winchester), while the second shooting session utilized a mixture of ammunition and firearms (Federal, Blazer, $9 \mathrm{~mm}$ and 40 caliber pistols). Moreover, a subset of 30 non-shooter background samples was monitored to assess potential false-positives and establish critical thresholds. All background samples resulted in 100\% true-negative rate, where all potential peaks were below LOD and LOQ. 
Table 4.6: Success rates of the baseline (no-activity) shooter samples in two separate shooting sessions using LC-MS/MS surface extraction procedure. The indicator " $N D$ " refers to analytes not detected.

\begin{tabular}{|c|c|c|c|c|c|c|c|}
\hline \multicolumn{2}{|c|}{ Session } & Analyte & Range (ppb) & Mean (ppb) & Median (ppb) & Positive Set & $\begin{array}{c}\text { Combined Constituent } \\
\text { data }\end{array}$ \\
\hline \multirow{11}{*}{1} & \multirow{7}{*}{$\begin{array}{l}\frac{1}{0} \\
0 \\
0\end{array}$} & AK2 & $0.50-296$ & 15.4 & 6.5 & \multirow{7}{*}{$\begin{array}{c}18 / 20 \\
90 \%\end{array}$} & \multirow{11}{*}{$\begin{array}{c}19 / 20 \\
95 \%\end{array}$} \\
\hline & & MC & \multicolumn{3}{|c|}{ ND } & & \\
\hline & & $\mathbf{E C}$ & $1.1-350$ & 13.8 & 2.8 & & \\
\hline & & DPA & $4.5-498$ & 102 & 74 & & \\
\hline & & N-NDPA & $6.8-271$ & 58 & 36 & & \\
\hline & & 4-NDPA & $3.7-30$ & 10.9 & 8.2 & & \\
\hline & & 2-NDPA & $6.2-58$ & 16.7 & 9.7 & & \\
\hline & \multirow{4}{*}{$\stackrel{\mathscr{0}}{\mathbb{B}}$} & \multirow{4}{*}{$\begin{array}{l}\mathrm{Ba} \\
\mathrm{Pb} \\
\mathrm{Sb}\end{array}$} & $\begin{array}{l}\text { Range } \\
\text { (ppm) }\end{array}$ & Mean (ppm) & Median (ppm) & \multirow{4}{*}{$\begin{array}{l}20 / 20 \\
100 \%\end{array}$} & \\
\hline & & & $1.1-8.9$ & 4.07 & 3.5 & & \\
\hline & & & $1.1-5.2$ & 1.83 & 1.4 & & \\
\hline & & & $6.1-10.1$ & 5.6 & 6.1 & & \\
\hline \multirow{11}{*}{2} & \multirow{7}{*}{ 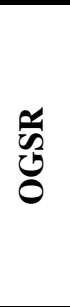 } & AK2 & $28-55$ & 42.8 & 42.1 & \multirow{7}{*}{$\begin{array}{l}33 / 75 \\
44 \%\end{array}$} & \multirow{11}{*}{$\begin{array}{l}75 / 75 \\
100 \%\end{array}$} \\
\hline & & MC & & ND & & & \\
\hline & & EC & $15.4-81.5$ & 42.1 & 40.3 & & \\
\hline & & DPA & $11.2-65.3$ & 30.1 & 25.1 & & \\
\hline & & N-NDPA & & ND & & & \\
\hline & & 4-NDPA & $9.3-38.2$ & 20.4 & 16.8 & & \\
\hline & & 2-NDPA & $9.1-57.9$ & 25.5 & 20.8 & & \\
\hline & \multirow{4}{*}{ 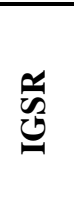 } & \multirow{4}{*}{$\begin{array}{l}\text { Ba } \\
\text { Pb } \\
\text { Sb }\end{array}$} & $\begin{array}{l}\text { Range } \\
\text { (ppm) }\end{array}$ & Mean (ppm) & Median (ppm) & \multirow{4}{*}{$\begin{array}{l}50 / 75 \\
66.7 \%\end{array}$} & \\
\hline & & & $1.7-4.5$ & 2.9 & 2.8 & & \\
\hline & & & $0.75-2.5$ & 1.5 & 1.2 & & \\
\hline & & & ND & ND & ND & & \\
\hline
\end{tabular}

Interestingly, the second session decreased overall OGSR and IGSR separate performance rates but demonstrated the GSR detection potential when combined (100\% true positive rate). These differences may be attributed a myriad of factors including the cartridge ejection port locations, composition of the powder and primers, or the randomness of each firing event. By using various ammunition formulae, the amounts and types of IGSR and OGSR deposited in hands was variable and at some extent, representative of casework.

The 78 activity samples were utilized to monitor the behavior of GSR for more realistic scenarios. These activities included vigorous hand rubbing, the application of hand sanitizer, and 
running for one minute after discharge events. These samples were cross-validated with LIBS and electrochemical methods and report the success rates and detection for LC-MS/MS compared to LIBS and electrochemistry, represented in Table 4.7. These results were compared to 10 baseline (no-activity) shooting samples and eight negative control (non-shooter) samples. The negative control samples were collected from the collection team before and after the shooting session to monitor any potential contamination.

Table 4.7: Success rates of activity samples (where $n$ is equal to the number of individuals) in separate shooting sessions. Additionally, the LC-MS/MS results were compared with LIBS and EC cross-reference results.

\begin{tabular}{|c|c|c|c|c|c|}
\hline & \multicolumn{2}{|c|}{ LC-MS/MS } & \multirow{2}{*}{$\frac{\text { LIBS }}{\text { IGSR (\%) }}$} & \multirow{2}{*}{$\frac{\text { LIBS + EC }}{\text { OGSR+IGSR }(\%)}$} \\
\hline Session & Activity & OGSR (\%) & OGSR+IGSR (\%) & & \\
\hline \multirow{5}{*}{1} & $\operatorname{Rub}(n=13)$ & 15.4 & 84.6 & 0 & 23.1 \\
\hline & Hand Sanitizer $(n=13)$ & 7.7 & 53.8 & 15.4 & 30.8 \\
\hline & Running $(n=13)$ & 15.4 & 23.1 & 15.4 & 15.4 \\
\hline & Baseline (no activity) $(n=5)$ & 100 & 100 & 60 & 100 \\
\hline & Background (non-shooters) $(n=4)$ & 0 & 0 & 0 & 0 \\
\hline \multirow{5}{*}{2} & $\operatorname{Rub}(n=13)$ & 30.8 & 84.6 & 46.2 & 92.3 \\
\hline & Hand Sanitizer $(n=13)$ & 69.2 & 92.3 & 38.5 & 84.6 \\
\hline & Running $(n=13)$ & 23.1 & 38.5 & 61.5 & 92.3 \\
\hline & Baseline (no activity) $(n=5)$ & 100 & 100 & 60 & 100 \\
\hline & Background (non-shooters) $(n=4)$ & 0 & 0 & 0 & 0 \\
\hline
\end{tabular}

Additionally, we saw the effect of weather and its role in the recovery of these analytes. The first shooting session involved inclement weather in the form of rain. The shooter's hands showed significant amounts of water when they entered the lab after the running activity. The results indicated the OGSR compounds were more affected versus the IGSR particulates ranging from 7.7-15.4\% for LC-MS/MS. This consideration presents another point when researching more "real-world" scenarios where weather could be impactful. For all samples, methyl centralite was not detected in any samples, which may be largely attributed to a shift in propellant formulation. On the other hand, we observed an increase in AK 2 detection in these items. 
Observing the analyte response versus activity under clearer weather conditions, the OGSR responses for the LC-MS/MS were more significant for the hand sanitizer versus hand rubbing and running. This may be primarily due to a "pseudo" organic extraction using isopropyl alcohol in direct contact with the hands. Conversely, running for $\sim$ one minute produced the lowest response for OGSR, which may be due to sweat formation allowing for the potential of increased absorption into the epidermal layer. Looking at the LIBS elemental responses, we observed an inverse relationship for the IGSR particulates where running was the greatest response followed by rubbing then hand sanitizer. This may indicate that the IGSR particulates are more influenced by physical interactions with hands in the forms of washing or sanitizing. Although these remarks and comments are speculative, the results illustrate the need to expand research efforts and interest in the persistence and fate of GSR analytes after a discharge event. Also, the preliminary postshooting activity data demonstrates that the detection of OGSR and IGSR by LC-MS/MS was feasible even for samples exposed to factors that decrease the chances for detection.

\subsection{Conclusions}

Herein, we demonstrate the use of host-guest chemistry to reach application in another discipline. Crown ethers are incredibly versatile not only for their transportation properties but also for their ability to retain isotopic ratios of the metals [35,41,70,71]. The method proved efficient for the detection of $\mathrm{Pb}$ and $\mathrm{Ba}$. On the other hand, the inability to detect antimony in authentic samples is primarily due to the chelated metal complex's detection limitations and ionization. Current research efforts focus on investigating optimal complexing agents to encompass IGSR analytes to lower the ionization potentials. Finding host molecules suitable for complexing all IGSR metals is inherently difficult because of the wide variety of complexing 
agents available, and the intricacies associated with the interactions between the metal species and host molecules.

More importantly, we investigated and validated an LC-MS/MS method to integrate into a broader workflow of GSR analysis. LC-MS/MS is a widely found technique in crime laboratories that primarily analyze drugs of abuse or toxicology samples. The figures of merit established represent this technique's capability but does not explicitly envelop all instrumentation models. This methodology's appealing aspect is the ability to use one sample for various screening tests like LIBS or electrochemistry followed by confirmatory techniques such as LC-MS/MS $[12,30,33]$. Following the ASTM E1588-20 guideline for GSR analysis allows this research to apply a universal collection substrate, compositional considerations, and an established standard instrument for reference.

However, without considering both components, the evidentiary value and understanding of GSR behavior may remain stagnant. The key to this method's successful deployment in the forensics field is to adopt LC-MS/MS approaches to increase confidence in the identification of GSR on a sample with both IGSR and OGSR information. The figures of merit presented demonstrate the ability of the method to identify and quantitate both OGSR and IGSR. With a further understanding and additional research concerning elemental and isotopic data when bound to host molecules, an instrumental technique like LC-MS/MS can provide additional quantitation for practitioners. Not only does this method increase the accuracy in GSR analysis and interpretation, but it yields an alternative avenue and resolves the need to choose between IGSR or OGSR. Finally, the approaches proposed in this study can be applied in other fields, monitoring exposure and environmental compartments near ammunition manufacturing or shooting facilities. 


\section{Author Contributions}

The manuscript was written through the contributions of all authors. All authors have given approval to the final version of the manuscript.

\section{Conflicts of interest}

The authors declare that they have no known competing financial interests or personal relationships that could have appeared to influence the work reported in this paper.

\section{Acknowledgments}

We would like to acknowledge both Dr. Keith Morris and James Hamilton for their support during the collection of authentic samples and access to the WVU ballistics laboratory. We would also like to acknowledge Kourtney Dalzell for her assistance in collection and analysis of the electrochemical results. Additionally, we would like to acknowledge Dr. Callee Walsh and Dr. Sandra Majuta for assistance and access to the orbitrap instrument in the BNRF.

This project is sponsored by Award No. \#2019-R2-CX-044 from the National Institute of Justice to West Virginia University. The opinions, findings, and conclusions are those of the authors and do not necessarily reflect those of the Department of Justice. 


\subsection{References for Chapter 4}

[1] T. Trejos, S. Koch, A. Mehltretter, Scientific foundations and current state of trace evidence-A review, Forensic Chem. 18 (2020) 100223. https://doi.org/10.1016/j.forc.2020.100223.

[2] L.S. Blakey, G.P. Sharples, K. Chana, J.W. Birkett, Fate and Behavior of Gunshot Residue-A Review, J. Forensic Sci. 63 (2018) 9-19. https://doi.org/10.1111/1556-4029.13555. [3] Z. Brozek-Mucha, Z. Brożek-Mucha, Trends in analysis of gunshot residue for forensic purposes, Anal. Bioanal. Chem. 409 (2017) 5803-5811. https://doi.org/10.1007/s00216-017$\underline{0460-1 .}$

[4] O. Dalby, D. Butler, J.W. Birkett, Analysis of Gunshot Residue and Associated Materials-A Review, J. Forensic Sci. 55 (2010) 924-943. https://doi.org/10.1111/j.15564029.2010.01370.x.

[5] M.A. de Carvalho, M. Talhavini, M.F. Pimentel, J.M. Amigo, C. Pasquini, S. Alves, I.T. Weber, M. Albino de Carvalho, M. Talhavini, M.F. Pimentel, J.M. Amigo, C. Pasquini, S.A. Junior, I.T. Weber, NIR hyperspectral images for identification of gunshot residue from tagged ammunition, Anal. Methods. 10 (2018) 4711-4717. https://doi.org/10.1039/c8ay01341a.

[6] F.S. Romolo, M.J. Bailey, J. de Jesus, L. Manna, M. Donghi, Unusual sources of Sn in GSR. An experimental study by SEM and IBA, Sci. Justice. 59 (2019) 181-189. https://doi.org/10.1016/j.scijus.2018.10.009.

[7] M. Donghi, K. Mason, F.S. Romolo, Detecting Gunshot Residue from Sellier \& Bellot Nontox Heavy Metal-free Primer by in situ Cathodoluminescence, J. Forensic Sci. 64 (2019) 1658-1667. https://doi.org/10.1111/1556-4029.14110. 
[8] K. Mason, R. Wuhrer, Phenom Desktop SEM for Gunshot Residue and

Cathodoluminescence Imaging and Analysis, Microsc. Microanal. 23 (2017) 1074-1075. https://doi.org/10.1017/s1431927617006031.

[9] S. Practice, S. Handbook, S. Practice, Standard Practice for Gunshot Residue Analysis by Scanning Electron Microscopy/Energy Dispersive X-Ray Spectrometry, ASTM Int. i (2020) 1-5. https://doi.org/10.1520/E1588-20.

[10] W. Feeney, C. Vander Pyl, S. Bell, T. Trejos, Trends in composition, collection, persistence, and analysis of IGSR and OGSR: A review, Forensic Chem. 19 (2020) 100250. https://doi.org/10.1016/j.forc.2020.100250.

[11] C. Bonnar, E.C. Moule, N. Lucas, K.E. Seyfang, R.P. Dunsmore, R.S. Popelka-Filcoff, K. Redman, K. Paul Kirkbride, Tandem detection of organic and inorganic gunshot residues using LC-MS and SEM-EDS, Forensic Sci. Int. 314 (2020) 110389. https://doi.org/10.1016/j.forsciint.2020.110389.

[12] T. Trejos, C. V Pyl, K. Menking-Hoggatt, A.L. Alvarado, L.E. Arroyo, Fast identification of inorganic and organic gunshot residues by LIBS and electrochemical methods, Forensic Chem. 8 (2018) 146-156. https://doi.org/10.1016/j.forc.2018.02.006.

[13] M. Lopez-Lopez, C. Alvarez-Llamas, J. Pisonero, C. Garcia-Ruiz, N. Bordel, An exploratory study of the potential of LIBS for visualizing gunshot residue patterns, Forensic Sci. Int. 273 (2017) 124-131. https://doi.org/10.1016/j.forsciint.2017.02.012.

[14] J. Coumbaros, K.P. Kirkbride, G. Klass, W. Skinner, Characterisation of 0.22 caliber rimfire gunshot residues by time-of-flight secondary ion mass spectrometry (TOF-SIMS): A preliminary study, Forensic Sci. Int. 119 (2001) 72-81. https://doi.org/10.1016/S0379-

\section{8(00)00421-7.}


[15] G. Vanini, R.M. Souza, C.A. Destefani, B.B. Merlo, T.M. Piorotti, E.V.R. de Castro, M.

Carneiro, W. Romao, Analysis of gunshot residues produced by .38 caliber handguns using inductively coupled plasma-optical emission spectroscopy (ICP OES), Microchem. J. 115 (2014) 106-112. https://doi.org/10.1016/j.microc.2014.03.003.

[16] M.I.A. Halim, M.F. Safian, E. Elias, S.S. Shazali, Ieee, Identification of Gunshot Residue from Trace Element by Using ICP/OES, 2013. \%3CGo.

[17] E. Goudsmits, G.P. Sharples, J.W. Birkett, Preliminary classification of characteristic organic gunshot residue compounds, Sci. Justice. 56 (2016) 421-425. https://doi.org/10.1016/j.scijus.2016.06.007.

[18] S.R. Khandasammy, A. Rzhevskii, I.K. Lednev, A Novel Two-Step Method for the Detection of Organic Gunshot Residue for Forensic Purposes: Fast Fluorescence Imaging Followed by Raman Microspectroscopic Identification, Anal. Chem. 91 (2019) 11731-11737. https://doi.org/10.1021/acs.analchem.9b02306.

[19] J. Bueno, I.K. Lednev, Attenuated Total Reflectance-FT-IR Imaging for Rapid and Automated Detection of Gunshot Residue, Anal. Chem. 86 (2014) 3389-3396.

https://doi.org/10.1021/ac4036718.

[20] D. Muller, A. Levy, A. Vinokurov, M. Ravreby, R. Shelef, E. Wolf, B. Eldar, B. Glattstein, A novel method for the analysis of discharged smokeless powder residues, J. Forensic Sci. 52 (2007) 75-78. https://doi.org/10.1111/j.1556-4029.2006.00309.x.

[21] B. Stevens, S. Bell, K. Adams, Initial evaluation of inlet thermal desorption GC-MS analysis for organic gunshot residue collected from the hands of known shooters, Forensic Chem. 2 (2016) 55-62. https://doi.org/10.1016/j.forc.2016.10.001. 
[22] C. Weyermann, V. Belaud, F. Riva, F.S. Romolo, Analysis of organic volatile residues in 9 mm spent cartridges, Forensic Sci. Int. 186 (2009) 29-35.

https://doi.org/10.1016/j.forsciint.2009.01.005.

[23] S. Benito, Z. Abrego, A. Sanchez, N. Unceta, M.A. Goicolea, R.J. Barrio, A. Sánchez, N. Unceta, M.A. Goicolea, R.J. Barrio, Characterization of organic gunshot residues in lead-free ammunition using a new sample collection device for liquid chromatography-quadrupole timeof-flight mass spectrometry, Forensic Sci. Int. 246 (2015) 79-85. https://doi.org/10.1016/j.forsciint.2014.11.002.

[24] A.L. Gassner, C. Weyermann, LC-MS method development and comparison of sampling materials for the analysis of organic gunshot residues, Forensic Sci. Int. 264 (2016) 47-55. https://doi.org/10.1016/j.forsciint.2016.03.022.

[25] A.M. O’Mahony, J.R. Windmiller, I.A. Samek, A.J. Bandodkar, J. Wang, "Swipe and Scan": Integration of sampling and analysis of gunshot metal residues at screen-printed electrodes, Electrochem. Commun. 23 (2012) 52-55. https://doi.org/10.1016/j.elecom.2012.07.004.

[26] A. Dona-Fernandez, I. de Andres-Gimeno, P. Santiago-Toribio, E. Valtuille-Fernandez, F. Aller-Sanchez, A. Heras-Gonzalez, A. Doña-Fernández, I. de Andres-Gimeno, P. SantiagoToribio, E. Valtuille-Fernández, F. Aller-Sanchez, A. Heras-González, Real-time detection of GSR particles from crime scene: A comparative study of SEM/EDX and portable LIBS system, Forensic Sci. Int. 292 (2018) 167-175. https://doi.org/10.1016/j.forsciint.2018.09.021. [27] L.A. Fambro, D.D. Vandenbos, M.B. Rosenberg, C.R. Dockery, Laser-Induced Breakdown Spectroscopy for the Rapid Characterization of Lead-Free Gunshot Residues, Appl. Spectrosc. 71 (2017) 699-708. https://doi.org/10.1177/0003702816689099. 
[28] E. Hondrogiannis, D. Andersen, A.W. Miziolek, The Evaluation of a New Technology for Gunshot Residue (GSR) Analysis in the Field, in: Conf. Next-Generation Spectrosc. Technol. VI, Spie-Int Soc Optical Engineering, BELLINGHAM, 2013: p. 87260P. https://doi.org/10.1117/12.2017900.

[29] M.B. Rosenberg, C.R. Dockery, Determining the Lifetime of Detectable Amounts of Gunshot Residue on the Hands of a Shooter Using Laser-Induced Breakdown Spectroscopy, Appl. Spectrosc. 62 (2008) 1238-1241. https://doi.org/10.1366/000370208786401473.

[30] Z. Abrego, N. Grijalba, N. Unceta, M. Maguregui, A. Sanchez, A. Fernandez-Isla, M.A. Goicolea, R.J. Barrio, A. Fernández-Isla, M.A. Goicolea, R.J. Barrió, A. Fernandez-Isla, M.A. Goicolea, R.J. Barrio, A novel method for the identification of inorganic and organic gunshot residue particles of lead-free ammunitions from the hands of shooters using scanning laser ablation-ICPMS and Raman micro-spectroscopy, Analyst. 139 (2014) 6232-6241. https://doi.org/10.1039/c4an01051e.

[31] M. Gallidabino, C. Weyermann, F.S. Romolo, F. Taroni, Estimating the time since discharge of spent cartridges: A logical approach for interpreting the evidence, Sci. Justice. 53 (2013) 41-48. https://doi.org/10.1016/j.scijus.2011.12.004.

[32] J.W. Moran, S. Bell, Skin Permeation of Organic Gunshot Residue: Implications for Sampling and Analysis, Anal. Chem. 86 (2014) 6071-6079. https://doi.org/10.1021/ac501227e. [33] L. Ali, K. Brown, H. Castellano, S.J. Wetzel, A Study of the Presence of Gunshot Residue in Pittsburgh Police Stations using SEM/EDS and LC-MS/MS, J. Forensic Sci. 61 (2016) 928-938. https://doi.org/10.1111/1556-4029.13077. 
[34] A. Tarifa, J.R. Almirall, Fast detection and characterization of organic and inorganic gunshot residues on the hands of suspects by CMV-GC-MS and LIBS, Sci. Justice. 55 (2015) 168-175. https://doi.org/10.1016/j.scijus.2015.02.003.

[35] S. Bell, W. Feeney, Single shot, single sample, single instrument detection of IGSR and OGSR using LC/MS/MS, Forensic Sci. Int. 299 (2019) 215-222.

\section{https://doi.org/10.1016/j.forsciint.2019.04.002.}

[36] W.A. MacCrehan, K.D. Smith, W.F. Rowe, Sampling protocols for the detection of smokeless powder residues using capillary electrophoresis, J. Forensic Sci. 43 (1998) 119-124. [37] M. Morelato, A. Beavis, A. Ogle, P. Doble, P. Kirkbride, C. Roux, Screening of gunshot residues using desorption electrospray ionisation-mass spectrometry (DESI-MS), Forensic Sci. Int. 217 (2012) 101-106. https://doi.org/10.1016/j.forsciint.2011.10.030.

[38] V. Redouté Minzière, D. Werner, D. Schneider, M. Manganelli, B. Jung, C. Weyermann, A.L. Gassner, Combined Collection and Analysis of Inorganic and Organic Gunshot Residues, J. Forensic Sci. (2020). https://doi.org/10.1111/1556-4029.14314.

[39] E. Bernal Morales, A.L. Revilla Vázquez, Simultaneous determination of inorganic and organic gunshot residues by capillary electrophoresis, J. Chromatogr. A. 1061 (2004) 225-233. https://doi.org/10.1016/j.chroma.2004.10.083.

[40] R. V Taudte, C. Roux, L. Blanes, M. Horder, K.P. Kirkbride, A. Beavis, The development and comparison of collection techniques for inorganic and organic gunshot residues, Anal. Bioanal. Chem. 408 (2016) 2567-2576. https://doi.org/10.1007/s00216-0169357-7. 
[41] S.M. Blair, E.C. Kempen, J.S. Brodbelt, Determination of binding selectivities in hostguest complexation by electrospray/quadrupole ion trap mass spectrometry, J. Am. Soc. Mass Spectrom. 9 (1998) 1049-1059. https://doi.org/10.1016/s1044-0305(98)00082-8.

[42] Y. Tao, R.R. Julian, Factors that influence competitive intermolecular solvation of protonated groups in peptides and proteins in the gas phase, J. Am. Soc. Mass Spectrom. 24 (2013) 1634-1640. https://doi.org/10.1007/s13361-013-0684-z.

[43] D.C. Reedy, C.S. Koper, Impact of handgun types on gun assault outcomes: A comparison of gun assaults involving semiautomatic pistols and revolvers, Inj. Prev. 9 (2003) 151-155. https://doi.org/10.1136/ip.9.2.151.

[44] Eurachem, The Fitness for Purpose of Analytical Methods, 1998. https://doi.org/978-9187461-59-0.

[45] K. Menking-Hoggatt, C. Martinez, C. Vander, E. Heller, E. Chip, L. Arroyo, T. Trejos, C. Vander Pyl, E. Heller, E. “Chip” Pollock, L. Arroyo, T. Trejos, Development of tailor-made inorganic gunshot residue (IGSR) microparticle standards and characterization with a multitechnique approach, Talanta. 225 (2021) 121984. https://doi.org/10.1016/j.talanta.2020.121984. [46] C.E. Ott, K.A. Dalzell, P.J. Calderón-Arce, A.L. Alvarado-Gámez, T. Trejos, L.E. Arroyo, Evaluation of the Simultaneous Analysis of Organic and Inorganic Gunshot Residues Within a Large Population Data Set Using Electrochemical Sensors*,†, J. Forensic Sci. 65 (2020) 1935-1944. https://doi.org/10.1111/1556-4029.14548.

[47] K. Menking-Hoggatt, L. Arroyo, J. Curran, T. Trejos, Novel LIBS method for microspatial chemical analysis of inorganic gunshot residues, J. Chemom. 35 (2021) 1-13. https://doi.org/10.1002/cem.3208. 
[48] M. Maitre, M. Horder, K.P. Kirkbride, A.L. Gassner, C. Weyermann, C. Roux, A.

Beavis, A forensic investigation on the persistence of organic gunshot residues, Forensic Sci. Int. 292 (2018) 1-10. https://doi.org/10.1016/j.forsciint.2018.08.036.

[49] D. Laza, B. Nys, J. De Kinder, A.K.D. Mesmaeker, C. Moucheron, Development of a quantitative LC-MS/MS method for the analysis of common propellant powder stabilizers in gunshot residue, J. Forensic Sci. 52 (2007) 842-850. https://doi.org/10.1111/j.15564029.2007.00490.x.

[50] E.M. Thurman, I. Ferrer, Adapting EPA Method 8330B for Analysis of Explosives in Water to SPE and LC/MS/MS Application Note, (2012) 1-8.

[51] G. Gaiffe, M.C. Bridoux, C. Costanza, R.B. Cole, A systematic tandem mass spectrometric study of anion attachment for improved detection and acidity evaluation of nitrogen-rich energetic compounds, J. Mass Spectrom. 53 (2018) 21-29. https://doi.org/10.1002/jms.4034.

[52] C. Bonnar, R. Popelka-Filcoff, K.P. Kirkbride, Armed with the Facts: A Method for the Analysis of Smokeless Powders by Ambient Mass Spectrometry, J. Am. Soc. Mass Spectrom. 31 (2020) 1943-1956. https://doi.org/10.1021/jasms.0c00193.

[53] M. Roberts, N. Petraco, M. Gittings, Novel method for the detection of nitroglycerin in smokeless powders, Sci. Justice. 55 (2015) 467-471. https://doi.org/10.1016/j.scijus.2015.08.001.

[54] S. Park, J. Lee, S.G. Cho, E.M. Goh, S. Lee, S.S. Koh, J. Kim, Mass spectrometric analysis of eight common chemical explosives using ion trap mass spectrometer, Bull. Korean Chem. Soc. 34 (2013) 3659-3664. https://doi.org/10.5012/bkcs.2013.34.12.3659. 
[55] D. Perret, S. Marchese, A. Gentili, R. Curini, A. Terracciano, E. Bafile, F. Romolo, LCMS-MS determination of stabilizers and explosives residues in hand-swabs, Chromatographia. 68 (2008) 517-524. https://doi.org/10.1365/s10337-008-0746-8.

[56] I. V. Kolesnichenko, E. V. Anslyn, Practical applications of supramolecular chemistry, Chem. Soc. Rev. 46 (2017) 2385-2390. https://doi.org/10.1039/c7cs00078b.

[57] S. Kafashi, M. Yaftian, A. Zamani, Binding Ability of Crown Ethers Towards Pb(II) Ions in Binary Water/Organic Solvents Using Solvent Extraction Method, J. Solution Chem. 44 (2015) 1798-1811. https://doi.org/10.1007/s10953-015-0378-1.

[58] N. Islam, S.S. Chimni, Binding and selectivity of phenazino-18-crown-6-ether with alkali, alkaline earth and toxic metal species: A DFT study, J. Mol. Struct. (2017). https://doi.org/10.1016/j.molstruc.2016.10.100.

[59] B. Yeager, K. Bustin, J. Stewart, R. Dross, S. Bell, Evaluation and validation of ion mobility spectrometry for presumptive testing targeting the organic constituents of firearms discharge residue, Anal. Methods. 7 (2015) 9683-9691. https://doi.org/10.1039/c5ay02417j. [60] M. Tella, G.S. Pokrovski, Antimony(III) complexing with O-bearing organic ligands in aqueous solution: An X-ray absorption fine structure spectroscopy and solubility study, Geochim. Cosmochim. Acta. 73 (2009) 268-290. https://doi.org/10.1016/j.gca.2008.10.014.

[61] A.B. Wijeratne, S.E. Spencer, J. Gracia, D.W. Armstrong, K.A. Schug, Antimony(III)-D, L-Tartrates Exhibit Proton-Assisted Enantioselective Binding in Solution and in the Gas Phase, J. Am. Soc. Mass Spectrom. 20 (2009) 2100-2105. https://doi.org/10.1016/j.jasms.2009.07.011. [62] A.B. Wijeratne, K.A. Schug, Molecular recognition properties of tartrates and metaltartrates in solution and gas phase, J. Sep. Sci. 32 (2009) 1537-1547. https://doi.org/10.1002/jssc.200900064. 
[63] J.M. Casas, G. Crisóstomo, L. Cifuentes, Antimony Solubility and Speciation in Aqueous Sulphuric Acid Solutions at 298 K, Can. J. Chem. Eng. 82 (2008) 175-183. https://doi.org/10.1002/cjce.5450820122.

[64] A.B. Wijeratne, S.H. Yang, D.W. Armstrong, K.A. Schug, Solvent Molecules Undergo Homolytic Cleavage and Radical Recombination Processes during Negative-Mode Electrospray Ionization: Adduct Formation with Antimony(III)-Tartrate Dianion, Anal. Chem. 82 (2010) 5141-5146. https://doi.org/10.1021/ac1003344.

[65] H.F. Lau, N.M. Quek, W.S. Law, J.H. Zhao, P.C. Hauser, S.F.Y. Li, Optimization of separation of heavy metals by capillary electrophoresis with contactless conductivity detection, Electrophoresis. 32 (2011) 1190-1194. https://doi.org/10.1002/elps.201000603.

[66] A.B. Wijeratne, J. Gracia, S.H. Yang, P. Kroll, D.W. Armstrong, K.A. Schug, New structural insight for antimony(III)-tartrate, Inorg. Chem. Commun. 13 (2010) 1504-1508. https://doi.org/10.1016/J.INOCHE.2010.08.028.

[67] D. Marcovich, R.E. Tapscott, 13C NMR Studies on Arsenic(III) and Antimony(III) Dihydroxydicarboxylate Complexes, J. Am. Chem. Soc. 102 (1980) 5712-5717. https://doi.org/10.1021/ja00538a002.

[68] R.C. Palenik, K.A. Abboud, G.J. Palenik, Bond valence sums and structural studies of antimony complexes containing Sb bonded only to O ligands, Inorganica Chim. Acta. 358 (2005) 1034-1040. https://doi.org/10.1016/J.ICA.2004.11.013.

[69] X. Li, T. Reich, M. Kersten, C. Jing, Low-Molecular-Weight Organic Acid Complexation Affects Antimony(III) Adsorption by Granular Ferric Hydroxide, Environ. Sci. Technol. 53 (2019) 5221-5229. https://doi.org/10.1021/acs.est.8b06297. 
[70] Y. Inokuchi, O. V. Boyarkin, R. Kusaka, T. Haino, T. Ebata, T.R. Rizzo, Ion selectivity of crown ethers investigated by UV and IR spectroscopy in a cold ion trap, J. Phys. Chem. A. 116 (2012) 4057-4068. https://doi.org/10.1021/jp3011519.

[71] V. Rudiger, H.J. Schneider, V.P. Solov’ev, V.P. Kazachenko, O.A. Raevsky, Crown ether-ammonium complexes: Binding mechanisms and solvent effects, European J. Org. Chem. (1999) 1847-1856. 


\section{CHAPTER 5}

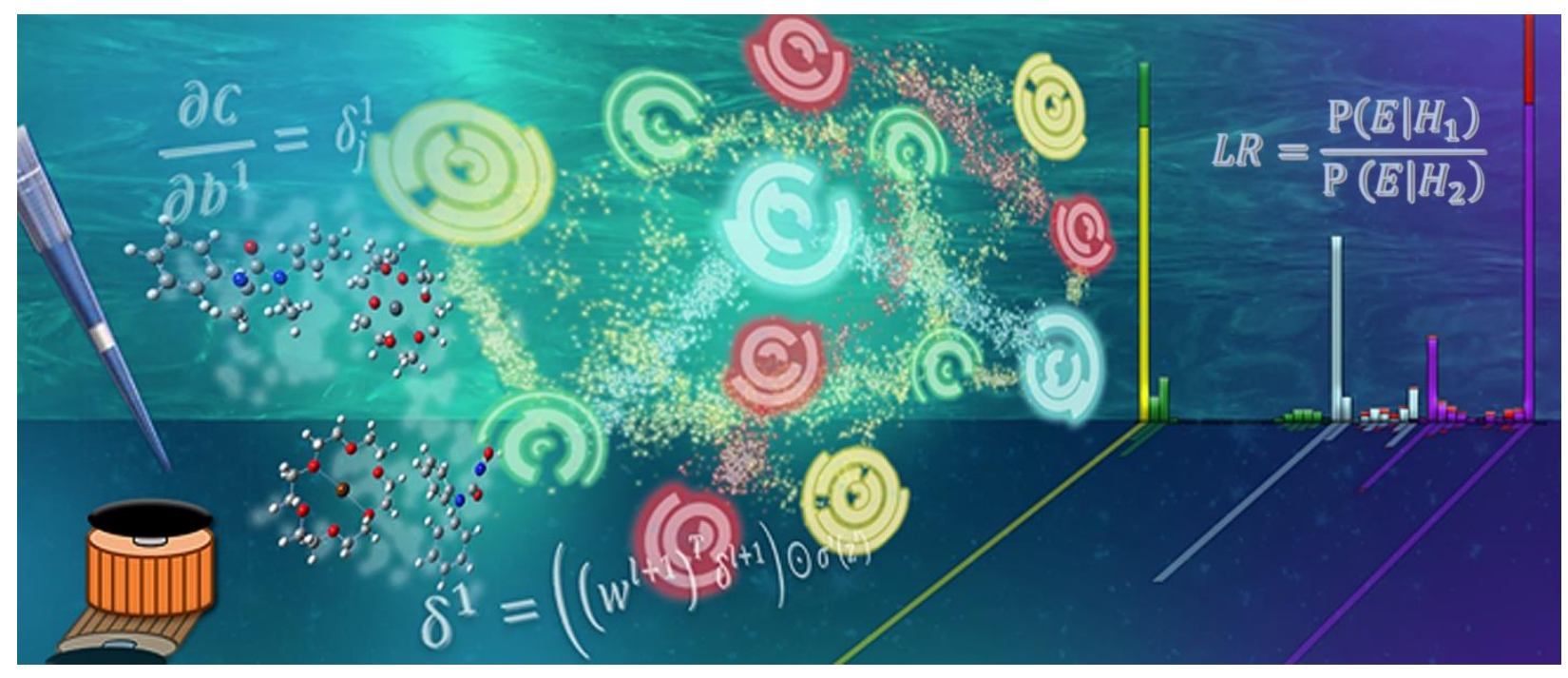

Evaluation of Organic and Inorganic Gunshot Residues in Various Populations using LC-MS/MS 
Overview: This work investigated the prevalence of organic and inorganic gunshot residue within two main subpopulations, 1) non-shooters, including groups with low- and high-risk of potentially containing GSR-like residues, and 2) individuals involved in a firing event (shooters, bystanders, and shooters performing post-shooting activities). The study analyzed over 400 samples via a liquid chromatography-mass spectrometry (LC-MS/MS) methodology with complexing agents. Exploratory statistical tools and machine learning algorithms (neural networks, $\mathrm{NN}$ ) were used to evaluate the resulting mass spectral and quantitative data. This study observed lower occurrences of OGSR compounds in the non-shooter populations compared to IGSR analytes. The presence of GSR on authentic shooters versus other potential sources of false positives, such as bystanders and professions including police officers, agricultural workers, and mechanics, were further assessed by utilizing machine learning algorithms trained with the observed OGSR/IGSR traces. The probability of false negatives was also estimated with groups who performed regular activities after firing. Additionally, the low-risk background set allowed documentation of GSR occurrence in the general population. The probabilistic outputs of the neural network models were utilized to calculate likelihood ratios (LR) to measure the weight of the evidence. Using both the IGSR and OGSR profiles, the NN model's accuracy ranged from 90 to $99 \%$, depending on the subpopulation complexity. The log-LR histograms and Tippet plots show the method can discriminate between each sub-population and low rates of misleading evidence, suggesting that the proposed approach can be effectively used for a probabilistic interpretation of GSR evidence. 


\subsection{Introduction}

\subsubsection{Current state and challenges of GSR detection}

Differentiation and classification of trace materials are critical tasks in many criminal investigations yet still present as a challenge for forensic science practitioners [1]. For firearmrelated cases, establishing relationships between a person of interest and a series of activities surrounding a shooting event is vital. Gunshot residues deposited on surfaces such as the victim, suspect, or crime scene may provide valuable information. A scientist must consider different aspects associated with transfer and persistence when interpreting the evidence as the collected samples are often recovered some time after the actual firing event. Likewise, it is important to understand how traces are formed and their commonality in the background environment.

GSR evidence is categorized into two constituents - inorganic particulates (IGSR) and organic compounds (OGSR). These analytes are formed after a firearm's firing pin strikes the primer of the ammunition (a shock sensitive explosive), which creates sparks and ignites, the propellant and expels a projectile at high velocities. When the deflagration event occurs, a plume comprised of burnt and unburnt residues disperses and interacts with objects in the immediate environment.

The nature of IGSR particulates and OGSR compounds leads to differences in both persistence and transfer mechanisms dependent on various factors, such as collection protocols, the randomness of the discharge event, the type of ammunition and firearm, and the substrate where the GSR deposit [2-5]. Additionally, both the presence and identification of GSR depend on the nature of the constituent of interest. For instance, the OGSR compounds are less prone to secondary transfer due to their volatility and lipophilic nature, their ability to be absorbed into the epidermal layer of the skin $[3,5,6]$. Conversely, the IGSR particulates adhere to the top layer of 
the skin and clothing but are more susceptible to secondary and even tertiary transfer when physically disrupted by mechanical forces $[4,5,7]$. These observations have led to numerous studies primarily geared towards the proper detection of IGSR particulates versus OGSR $[2,4,8]$. Currently, IGSR is measured using Scanning Electron Microscopy Energy Dispersive Xray Spectrometry (SEM/EDS) based on the standard guideline ASTM E1588-20 [9]. The ASTM framework for interpretation of gunshot residues is based on a categorical approach and relies upon particle morphology and elemental composition to describe the confidence when determining GSR versus GSR-like particles from environmental sources [9]. The morphological characteristics of an IGSR particulate typically include sizes ranging from $0.5 \mu \mathrm{m}$ to $5.0 \mu \mathrm{m}$ in diameter and possess a spheroid structure [9]. The chemical compositions further comprises three categories based on elements observed; 1) characteristic of GSR - lead $(\mathrm{Pb})$, barium $(\mathrm{Ba})$, and antimony $(\mathrm{Sb})$ are all present within a particle, 2) consistent with GSR - combinations such as $\mathrm{PbBa}$ or $\mathrm{BaSb}$, and 3) commonly associated with GSR - the presence of particles with one element such as $\mathrm{Ba}, \mathrm{Pb}$, or $\mathrm{Sb}$, listed from most to least discriminating classification [9].

Nonetheless, this categorical interpretation approach becomes problematic when particles do not exhibit spheroid properties or contain unusual profiles like heavy metal-free ammunition, which can vary greatly, unlike leaded ammunition [10]. Moreover, the method does not provide an assessment of the weight of the evidence. As a result, instrumental techniques such as LIBS, LA-ICP/MS, Raman, and TOF-SIMS have been explored to complement information provided by SEM/EDS [5,11,12]. Likewise, there has been an increased interest in evaluating the benefit of using OGSR compounds information to develop consensus-based guidelines [13]. Currently, gas (GC) and liquid chromatography (LC) coupled to mass spectrometers (MS) are widely accepted 
techniques that inspect both burnt and unburned propellant and powder remnants after a deflagration event $[5,14-16]$.

Proposed alternatives have been reported incorporating inorganic and organic information to increase confidence in collected samples to suit casework needs [17-20]. These approaches have included cost-effective, rapid screening techniques such as Raman, electrochemistry, and FTIR for simultaneous analysis [18,21-23]. The alternative to these "one-step" methods is merging instruments that benefit from lower critical thresholds, detection, and quantitation limits. These combinations include LIBS with electrochemistry, CMV-GC/MS with LIBS, and LCMS/MS with SEM/EDS [19,24-26]. Recently, a confirmatory analysis technique (LC-MS/MS) was adapted to quantify and identify IGSR and OGSR using chromatographic approaches in conjunction with host-guest chemistry [17]. That study highlights factors like varying ammunition formulations and weather conditions that may influence the detection of analytes.

\subsubsection{Population considerations}

An overarching concern for GSR analysts is the transfer and persistence of analytes within the general population and the risk of potential false-positives and cross-contamination [3,27-29]. Since a particle may not possess a typical "spheroid" shape, GSR analysts need to rely more on the chemical composition $[9,30,31]$. Because physical interactions affect IGSR particles more than OGSR compounds, some studies have considered observing possible sources and transfer rates from apprehending officers, the surfaces in police stations, and even their patrol cars [24,32-36]. Additionally, several studies have noted that other professions and individuals who work consistently with materials such as brake pads and heavy machinery (mechanics and agricultural workers) possess particles with similar chemical compositions [37-39]. For instance, brake pads rely on friction and thus need to be coated with metal sulfides like antimony trisulfide $\left(\mathrm{Sb}_{2} \mathrm{~S}_{3}\right)$ to 
stabilize the heat and act as a lubricant [37]. For agricultural workers, the chemicals used for leadbased paints and heavy machinery can resemble the elemental signatures of GSR and may be transferred due to aging or application, making them common sources of heavy metals.

The occurrence of IGSR in the environment has been extensively studied whereas the same cannot be stated for OGSR [5,27,40-42]. However, it is known that two common OGSR analytes, nitroglycerin and diphenylamine, also appear in products ranging from pharmaceutical uses to improve blood flow, or dyes, fungicides, and industrial antioxidants, respectively [41]. Such information has sparked investigations into estimating the prevalence of other OGSR compounds in background (known non-shooter) populations [3,23,28,43,44]. Hence, these scenarios may influence future decisions to incorporate OGSR evidence with IGSR information when investigating a firearm-related event [12,17-19,23,25,26]. Additionally, knowledge of the prevalence of OGSR in populations of interest is critical in any (statistical) evaluation of the weight of evidence. It is unsurprising that the presence of a GSR compound should be considered stronger evidence if its prevalence in the non-shooter population is low, and weak evidence if its prevalence in the non-shooter population is high.

\subsubsection{Neural Networks and likelihood ratios for GSR interpretation}

Combining OGSR and IGSR increases confidence in identification of firearm discharge residues and classification of results into populations such a shooter, non-shooter, and more complicated scenarios in the case of bystanders and high-risk individuals [5,15,17,19,21]. Previously, our group employed a categorical criterion for identifying a GSR-containing sample. This criterion labeled a sample positive for GSR when at least three combined OGSR/IGSR components were detected per sample (i.e., two OGSR compounds and one IGSR analyte or vice 
versa) $[5,15,17,19,21]$. However, with more complex samples from various populations, more comprehensive approaches are needed for accurate and rapid classification.

Numerous strategies have been proposed for this purpose including artificial intelligence, case-by-case interpretation approaches, likelihood ratios, and Bayesian networks $[2,23,51,52,28,29,45-50]$. Our group has explored using complex machine learning methods, such as neural networks $(N N)$, and widely used machine learning methods, such as logistic regression models to obtain probabilistic classifications based on GSR evidence [12,23]. Neural networks are linked mathematic structures designed to predict the class of a questioned sample by first training the network using representative data sets of known samples [28,53]. Training occurs by iterative adjustment of coefficients (weighting factors) incorporated within one or more well-understood mathematical functions [53]. Cross-validation can be used to estimate how the model accuracy will vary, and hence how the model might perform with unseen data - i.e., data not used for training. Both neural networks and logistic regression give probabilities which can be used to generate likelihood ratios.

Likelihood ratios in GSR-related research measures the weight of the evidence and are used to evaluate the probability of detecting GSR given two mutually exclusive hypotheses [48,51]. The calculation of the LR is represented by Equation 5.1

$$
L R=\frac{\mathrm{P}\left(E \mid H_{1}\right)}{\mathrm{P}\left(E \mid H_{2}\right)}
$$

Equation 5.1

Where $E$ is the evidence, i.e., the GSR, and $H_{1}$ and $H_{2}$ are mutually exclusive explanations for the presence of the evidence. If $H_{1}$ and $H_{2}$ are formulated at the source level [54], the $H_{1}$ may be the hypothesis is that traces originated from gunshot (and hence, are GSR) whereas $\mathrm{H}_{2}$ represents the hypothesis that the traces originated from some non-firearm source (and hence, are not GSR). The 
further LR is above 1, the greater the support for identifying the traces as GSR $\left(H_{1}\right)$ and vice versa. As an example, if the LR is 100 , then the evidence is 100 times more likely if the trace is GSR than if it is not. Conversely, if the LR is 0.01 , then the evidence is 100 times more likely if they are not GSR ${ }^{1}$.

This study aims to increase the existing knowledge on IGSR and OGSR in various subpopulations. It also aims to provide a statistical interpretation of the evidence using a Bayesian (or likelihood ratio) based approach. Statistical interpretations can lead to more objective decisions and assessment of the significance of the evidence. A Bayesian approach allows the scientist to contrast the value of the evidence with respect to at least two competing explanations. This approach is compatible with the legal framework to provide the prosecution and the defense a testimony on the value of the evidence that is easily interpreted and does not over or understate the said value. Additionally, the previously validated LC-MS/MS work will be tested for its accuracy and discriminating power for GSR-based evidence while also assessing the probability of finding analytes of GSR in the environment through a neural network model.

\subsection{Materials and Methods}

\subsubsection{Consumables}

Optima ${ }^{\circledR}$ LC/MS grade methanol $(\mathrm{MeOH})$, acetonitrile $(\mathrm{ACN})$, and water $(\mathrm{H} 2 \mathrm{O})$, all containing $0.1 \%$ formic acid (FA), were obtained from Thermo-Fisher Scientific (Watham, MA) and used either as extraction solvents or mobile phases. Standard (neat) organic constituents used in this study included: akardite II (AK 2), ethyl centralite (EC), methyl centralite (MC), diphenylamine (DPA), N-nitrosodiphenylamine (N-NDPA), 2-nitrodiphenylamine (2-NDPA), and 4-nitrodiphenylamine (4-NDPA) were obtained from Sigma-Aldrich and Accustandard®

\footnotetext{
${ }^{1}$ It is traditional when the LR is less than one to take the reciprocal and provide the interpretation with respect to $H_{2}$.
} 
(New Haven, CT). Calibration curves were generated from working stock solutions and diluted in $\mathrm{MeOH}(\geq 95 \%)$. Complexing agents 18-crown-6-ether (18C6) and tartaric acid (TT) were purchased from Sigma-Aldrich (St. Louis, MO 99\% purity) and diluted using $\mathrm{MeOH}$ and water respectively. Metal IGSR standards used to form complexes utilized ICP-MS metal standards (VHG Laboratories, Manchester, $\mathrm{NH})$ and methanol. Micro-bulk digestions utilized nitric $\left(\mathrm{HNO}_{3}\right)$ and hydrochloric $(\mathrm{HCl})$ acids (ultrapure grade, Thermo-Fisher Scientific). Two internal standards were used throughout the study, including deuterated diphenylamine-d10 (D10-DPA), which was purchased from CDN Isotopes (Quebec, Canada) and used for the organic molecules and thallium (Tl) for the IGSR (VHG Laboratories, Manchester, NH) and complexing agents.

\subsubsection{Firearms, ammunition, and protocols}

Two different firearms were used to collect authentic shooter samples: a $9 \mathrm{~mm}$ Springfield XD9 semi-automatic pistol and a 40 caliber Springfield XD40 semi-automatic pistol. The ammunition was either loaded by the manufacturer (Remington®, Blazer®, Federal ${ }^{\circledR}$ ) or loaded in-house (Winchester $\left.{ }^{\circledR}, \mathrm{CCI}{ }^{\circledR}\right)$. Each specialty ammunition consisted of brass Starline ${ }^{\mathrm{TM}}$ cartridge cases loaded with either Winchester ${ }^{\circledR}$ or CCI ${ }^{\circledR}$ small pistol primers, Winchester ${ }^{\circledR} 231$ propellant, and Speer® $9 \mathrm{~mm}$ full metal jacketed bullets. All firing events were performed at the indoor WVU ballistics laboratory.

The shooters discharged five consecutive shots inside the WVU ballistics range. After firing, the firearm was cleared and placed with the range officer. The shooters then proceeded to the collection laboratory, where both left- and right hands (palm and back) were stubbed $\sim 15$ times in the areas from the index finger to the thumb. After sample collection, the shooters washed their hands and repeated the process. The collection of samples was conducted in a separate room. Before each session, working areas were cleaned and covered with butcher paper. Negative control 
samples were obtained from the collection team to establish any potential presence of constituents. Additionally, the collection team members and shooters dressed in Tyvek® suits for additional protection from carryover.

For the post-shooting activity data set, one of three actions were performed after five successive discharges, including hand rubbing, hand sanitizer application, running, and running in the rain. For hand rubbing, the subjects clasped both hands together and vigorously rubbed for approximately 30 seconds. After that time, the collectors proceeded to stub the subjects' index and thumb areas. The second activity followed a similar motion as the first activity with a hand sanitizer application. For the third activity, the shooter exited the range and proceeded to run outdoors for approximately 60 seconds. The collectors then proceeded to stub their hands after they entered the laboratory.

For the bystander population set, volunteers stood in the relative vicinity of a discharge event (approximately 3-feet) at three varying locations (left, right, and behind the shooter) illustrated in Figure S5.1. Similarly, after 5 shots, the firearm was cleared and placed with the range officer, to which the bystanders proceeded to the collection laboratory, and both hands (palm and back) were stubbed 15 times. Immediately following, the shooter's hands were stubbed to gather positive controls of the firing event. After sample collection, both bystanders and shooters washed their hands and repeated the process. After the firing session concluded, the collection team obtained additional negative control samples from the collectors to test potential secondary transfers or unintended contamination. Once all samples were stored, the Tyvek® suits, parchment paper, and gloves were discarded.

For both the high-risk and low-risk sample sets, volunteers were asked a series of questions concerning their activities within the past 24 hours. The questions centered around recent firearms 
handling and participation in activities such as welding, agricultural, or mechanic work activities (i.e., body shop, brake repairs). If the answer was no, the sample was classified as low-risk and inversely labeled as high-risk if yes. No personal information was collected from the volunteers, and both hands (palm and back) were stubbed $\sim 15$ times.

\subsubsection{Sample extraction and collection}

Samples were collected following the ASTM E1588-20 guideline [9] and stored in a $-20^{\circ} \mathrm{C}$ freezer to prevent sample loss and cross-contamination. A total of two samples are generated from a single firing event, including both dominant and non-dominant hands. The extraction and instrumental parameters followed the same methodology outlined in our previous publication [17].

\subsubsection{Population study}

The population study consists of hand swabs collected from over 400 individuals and approved by the Institutional Review Board (WVU IRB protocol \#1506706336). The collection team sampled from various Morgantown, WV areas, including local police stations, agricultural greenhouses, and graduation events. Of the 75 low-risk individuals, none reported discharging a firearm within 48 hours preceding sampling. The high-risk samples were collected within the ballistics research laboratory at West Virginia University from those individuals either actively handling cartridges, reloading ammunition, or within the same working areas as the individuals handling firearm-related equipment (20 volunteers). For the remaining samples of the high-risk population, 34 police officers were sampled from the headquarters of the West Virginia University police, 18 mechanics samples were collected from several "auto repair" shops located in and around the Morgantown area, and 21 agricultural worker samples originated from farms and greenhouses owned by WVU and were located on- and off-campus. Table 5.1 further describes the number of samples contributing to the overall sub-population sets. 
Table 5.1: General breakdown of each population analyzed by LC-MS/MS analysis.

\begin{tabular}{|c|c|c|c|}
\hline \multicolumn{2}{|r|}{ Group } & Population subgroup and description & \multirow{2}{*}{$\begin{array}{c}\begin{array}{c}\text { \# of } \\
\text { persons }\end{array} \\
75\end{array}$} \\
\hline 1 & $\begin{array}{c}\text { Low-Risk } \\
\text { Background }\end{array}$ & $\begin{array}{l}\text { General population- Populous at WV who have not handled a firearm (WVU } \\
\text { students, staff, and visitors) }\end{array}$ & \\
\hline & & Low-Risk Samples Total & 75 \\
\hline \multirow{4}{*}{2} & \multirow{4}{*}{$\begin{array}{l}\text { High-Risk } \\
\text { Non-shooters }\end{array}$} & Police officers - Local law enforcement & 34 \\
\hline & & Mechanics - Professionals dealing with brake linings and vehicle parts & 18 \\
\hline & & Agricultural workers - Individuals who work within farms and greenhouses & 21 \\
\hline & & Ballistics research personnel- Researchers that work with or near firearms & 20 \\
\hline & & High-Risk Samples Total & 93 \\
\hline \multirow{3}{*}{3} & \multirow{3}{*}{$\begin{array}{l}\text { Authentic } \\
\text { shooter } \\
\text { samples }\end{array}$} & Bystander - Individuals near a firing event & 75 \\
\hline & & Baseline shooters (no-activity) - Individuals firing a gun & 75 \\
\hline & & $\begin{array}{l}\text { Post-shooting activity - Individuals firing a weapon followed by various } \\
\text { activities before collection }\end{array}$ & 78 \\
\hline \multicolumn{3}{|r|}{ Authentic shooter Samples Total } & 228 \\
\hline 4 & $\begin{array}{l}\text { Negative } \\
\text { Controls }\end{array}$ & $\begin{array}{l}\text { Collection Team - Samples from individuals who collect from all population } \\
\text { sets }\end{array}$ & 20 \\
\hline \multicolumn{3}{|r|}{ Negative Controls Total } & 20 \\
\hline \multicolumn{3}{|r|}{ Sum of all subpopulations } & 416 \\
\hline
\end{tabular}

Again, no personal information was taken from the volunteers. The information documented is related to the physical characteristics and objects on the hands and their dominant hand. Examples of physical features and objects include hand dryness, lotion, visible soil and contaminants, cuts, wearing rings, etc.

\subsubsection{Data analysis}

Data analysis involved various steps in confirming the presence of analytes before constructing the statistical model. After data acquisition, the data was processed by the Agilent MassHunter® Quantitative Analysis software, exported to Microsoft Excel®, and manually evaluated for quality control. Each analyte was checked to ensure that the response was above LOD. The sequential steps involved comparing each response with the calibration curve controls collected the same day, and samples were labeled with an automated code to identify if each analyte was below or above the respective LOD and LOQ thresholds. The concentration values 
were then used for exploratory statistical analysis and input data for the neural network model and subsequent likelihood ratio calculations.

\subsubsection{Machine Learning model}

The LC-MS/MS data were transformed into quantitative values via external calibration curves with internal standard, and analyzed to train, validate, and test the neural network algorithm. The classification outputs were compared to targets through dynamic programming via a backpropagation strategy in which the weights improve the prediction [28,53]. The backpropagation processes the inputted data numerous times using differential calculus until a user-desired minimum error is achieved or the root mean square (RMS) error across the network is negligible.

Before inputting data for $\mathrm{NN}$ evaluation, data was preprocessed using feature normalization strategies such as min-max normalization (scaling all values on a $0-1$ relative scale) and z-scoring (centering on the mean and scaling based on standard deviation values. In addition to producing unitless input values, scaling is essential to avoid over-emphasis on large concentration values and underrepresentation of low, but still significant, concentration values. Once trained, the network then can be evaluated using data that is separate from the training set, including the validation and testing sets. For samples with concentration values below the LOD, those values were treated as "0".

For this study, min-max normalization was utilized as it produced the best results and the data was split randomly using a 60:20:20 ratio - 60\% for training, $20 \%$ for validation, and $20 \%$ for testing. The JMP Pro® statistical software version 14.0.0 and R Studio @ (version 1.4.1717) were used to construct the neural networks and estimate the likelihood ratios. Also, we followed a similar criterion and mimicked the previous publication to estimate the positive responses of the 
analytes, using a categorical approach considering a positive sample when detecting at least two OGSR compounds and one IGSR analyte or vice versa [17]. This categorical criterion was used to estimate performance rates, as compared to the $N N$ approach.

\subsection{Results and Discussion}

\subsubsection{Occurrence of OGSR and IGSR on the sampled populations}

\subsubsection{Comparison of low-risk, high-risk, and shooter sets}

Exploratory data analysis methods, such as descriptive statistics (Table S5.1) and boxplots, compared the distribution of analytes in the five populations and were used to elucidate OGSR and IGSR patterns. The baseline shooters (no-activity) and low-risk (background) populations served as upper and lower limits for the remaining populations due to their substantial concentration differences (Figures 5.1-5.3). The low-risk (background) population displayed little to no OGSR or IGSR concentrations above detection or quantitation limits barring ethyl centralite (EC) which only showed a signal above LOD (1.0 ppb) but below LOQ for approximately $42 \%$ of the samples.

Again, minimal to no OGSR signals were observed in the high-risk set consisting of hand samples of police officers, mechanics, and farmhands, except for EC (Figure 5.1). However, the IGSR signals were higher for barium $(\mathrm{Ba})$ and lead $(\mathrm{Pb})$ compared to the low-risk population. For the high-risk individuals, signals for $\mathrm{EC}, \mathrm{Ba}$, and $\mathrm{Pb}$ were observed on approximately $40 \%, 90 \%$, and $34 \%$, respectively. Of those samples, only $34 \%$ contained two analytes ( $\mathrm{Ba}$ and $\mathrm{Pb}$ ), while only $9 \%$ possessed a combination of three constituents (EC, $\mathrm{Ba}$, and $\mathrm{Pb}$ ) on a single sample. These results indicate that IGSR is more prevalent than OGSR amongst this subgroup and that the combined detection of IGSR/OGSR decreased the number of false positives.

In contrast, samples collected from baseline shooters shortly after discharging a firearm presented both IGSR and OGSR at significantly higher concentrations than the low and high-risk 
sets (Hotelling's T-squared). All shooter stubs possessed a combination of at least three combined OGSR/IGSR analytes, with $100 \%$ of the samples presenting $\mathrm{EC}, \mathrm{Ba}$, and $\mathrm{Pb}$ at higher concentrations. Additional OGSR compounds were detected in shooter samples alongside EC including: 2-NDPA (36\%), DPA (29\%), 4-NDPA (24\%), AK2 (16\%), and N-NDPA (7\%). None of the shooter samples showed detectable levels of methyl centralite which may be a result of a difference in ammunition formulation by substituting in AK2, as observed in the respective safety datasheets of the used ammunition.

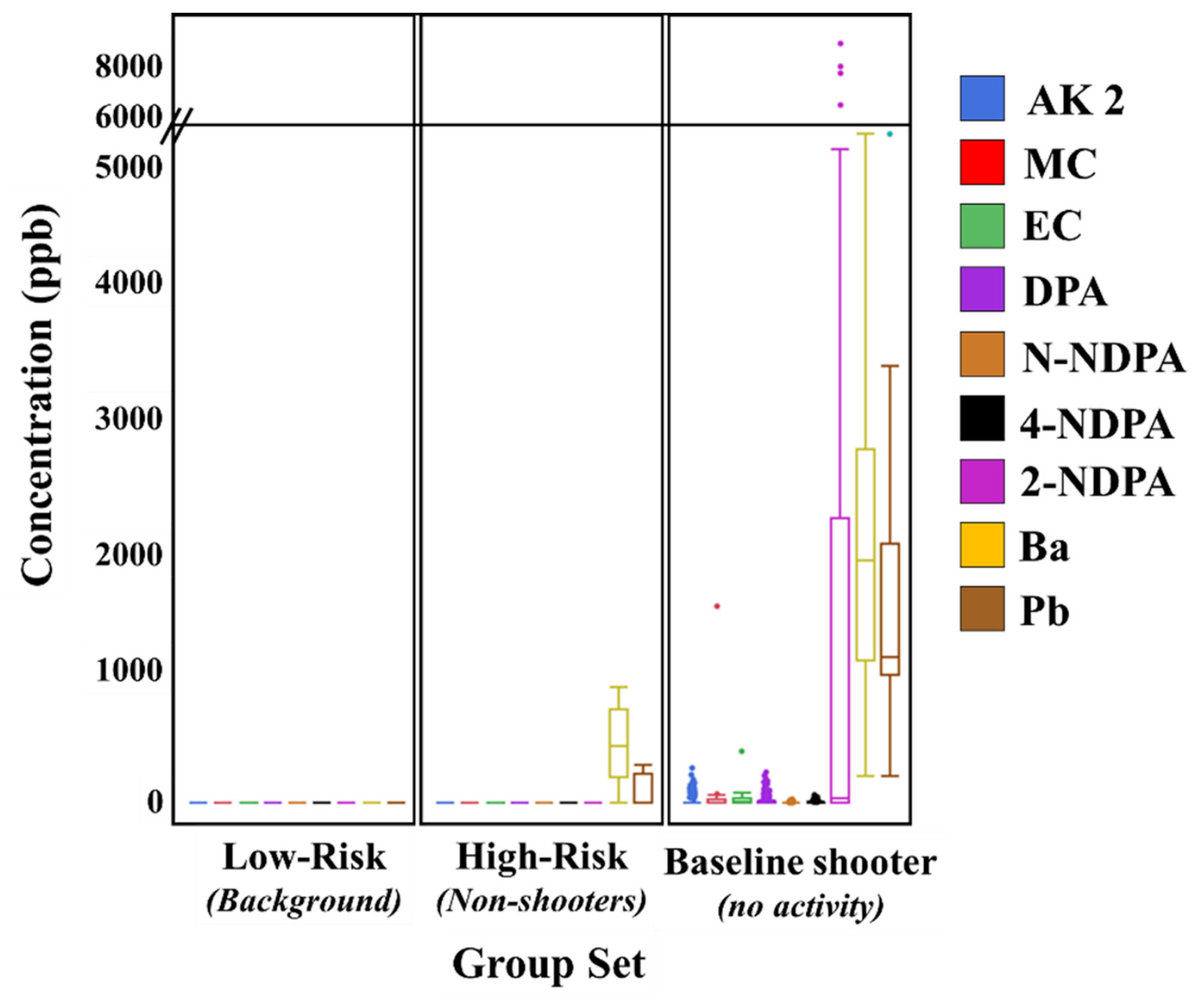

Figure 5.1: Boxplot comparisons between three populations. Displayed are the low-risk (background samples), the high-risk and baseline shooter (no activity) responses for seven OGSR, and two IGSR studied analytes. 
All the baseline shooter samples contained $\mathrm{EC}$ at an average concentration of $38 \mathrm{ppb}$ versus $0.6 \mathrm{ppb}$ and $1.0 \mathrm{ppb}$ for low- and high-risk sets, respectively. Only $43 \%$ of these samples possessed at least two OGSR compounds, with EC being the most frequent, followed by 2-NDPA. Additionally, $26 \%, 24 \%$, and $22 \%$ of the shooter samples contained at least three, four, or five OGSR compounds on the same stub, respectively.

\subsubsection{Post-shooting activity}

The second subpopulation consisted of individuals who discharged a firearm and performed one of three activities immediately after the event to mimic "real-world" scenarios (Figure 5.2). These post-shooting activities involved 1) vigorously rubbing the hands together for 30 seconds, 2) applying hand sanitizer and rubbing for 30 seconds, or 3) running outdoors for approximately 1 minute. As expected, the post-shooting activity samples demonstrated higher signals of both IGSR and OGSR compared to the low-risk backgrounds but lower levels than the baseline shooter responses. This observation indicates a significant loss of analyte signal but not a complete depletion of the IGSR/OGSR. Out of the three activities, the hand sanitizer collected a significant amount of OGSR analytes. One possible explanation for this observation is the organic

pseudo-extraction caused by applying isopropanol directly on the areas, producing a preconcentration effect before stubbing the hands. Out of the remaining activities running produced the lowest responses for OGSR, which may result from sweat formation allowing for the potential of increased absorption into the epidermal layer and/or more loss from evaporation due to an increase in body temperature [17]. 


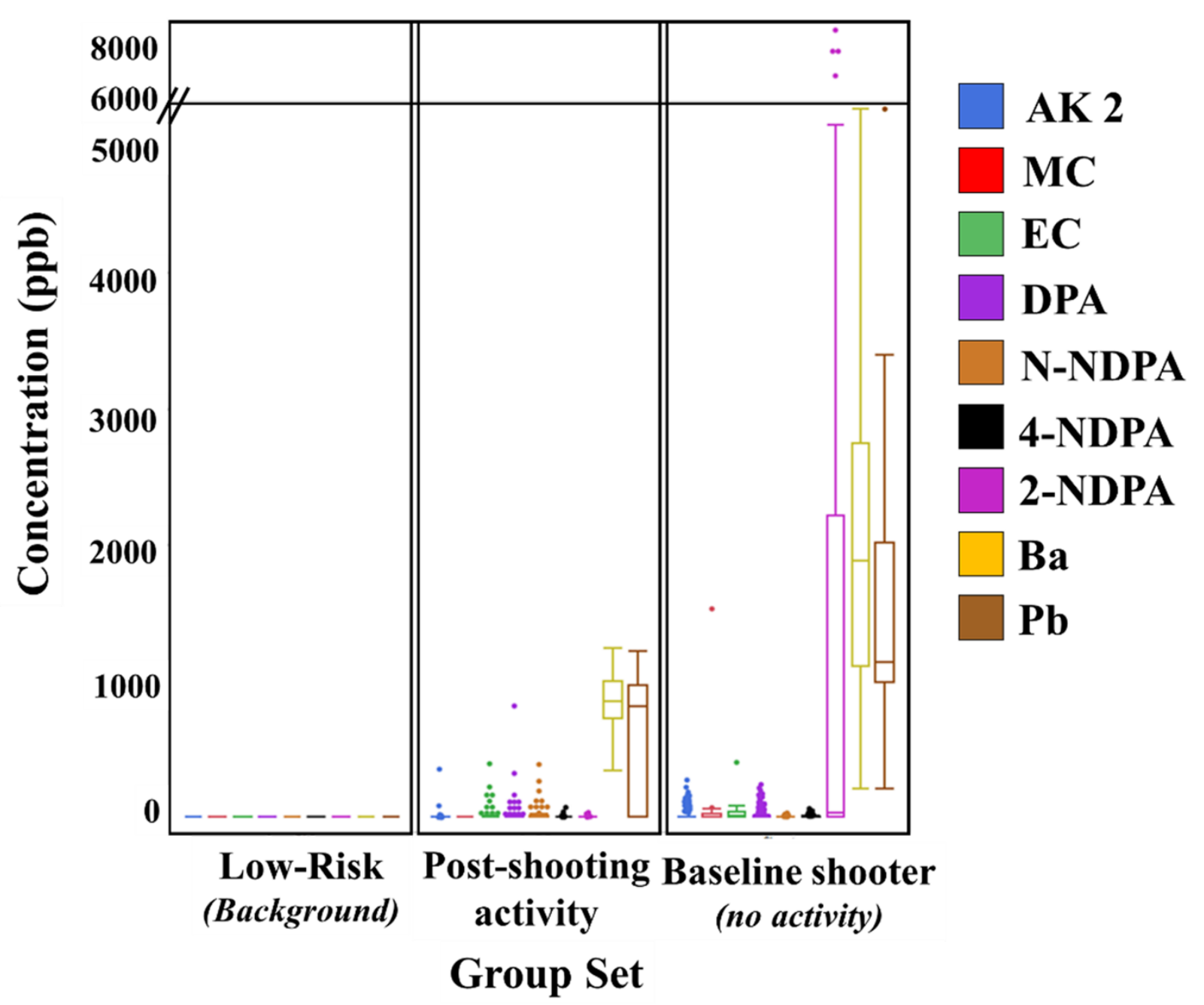

Figure 5.2: Boxplot comparisons between three populations. The low-risk (background samples), the post-shooting activity, and baseline shooter (no activity) responses for seven OGSR and two IGSR studied analytes are displayed.

To accurately compare this set with baseline shooting samples, positive control samples (shooter without activity) were collected on the same days for quality control monitoring. These positive controls showed a statistically significant difference (Hotelling's T-squared) for all OGSR compounds, barring 4-NDPA, once an activity was performed. However, there was no statistical difference between the IGSR signals of the positive controls and the activity samples. This result may be due to evaporation rates of the OGSR compounds as compared to more persistent IGSR particulates. Among the seven studied OGSR compounds, the concentration levels decreased 
anywhere from $76 \%-99 \%$, with AK 2 experiencing the most significant loss. This observation further illustrates that OGSR components are more sensitive to factors like weather conditions, skin absorption, and/or temperature [6,55]. Although the activities yielded lower concentrations of the analytes of interest, IGSR elements were detected on all samples and $88 \%$ presented more than two OGSR compounds.

\subsubsection{Bystander}

For the last subpopulation set, we wanted to evaluate the levels of GSR that may be deposited on individuals within the vicinity of a discharge event. Because of the ballistics range's dimensions and safety concerns, bystanders were oriented at three different locations (left, right, and behind) approximately three feet away from the shooter. Very few GSR compounds were detected in the bystander population set (Figure 5.3), except for Ba (94\% of the samples) and EC (12\% of the samples). Comparing these findings to the Low-Risk and negative control samples, it appears that both analytes are originating from the discharge event. Another important observation is the presence of barium being more prevalent than lead in this subpopulation. This may be attributed to the crown ethers being more sensitive to barium metal [17] and the amount of lead being below the LOD. However, these observations may have been influenced by a myriad of factors, including the shooting range conditions, the time gap between firing and sampling, and the positioning of the bystanders in respect to the discharge event. 


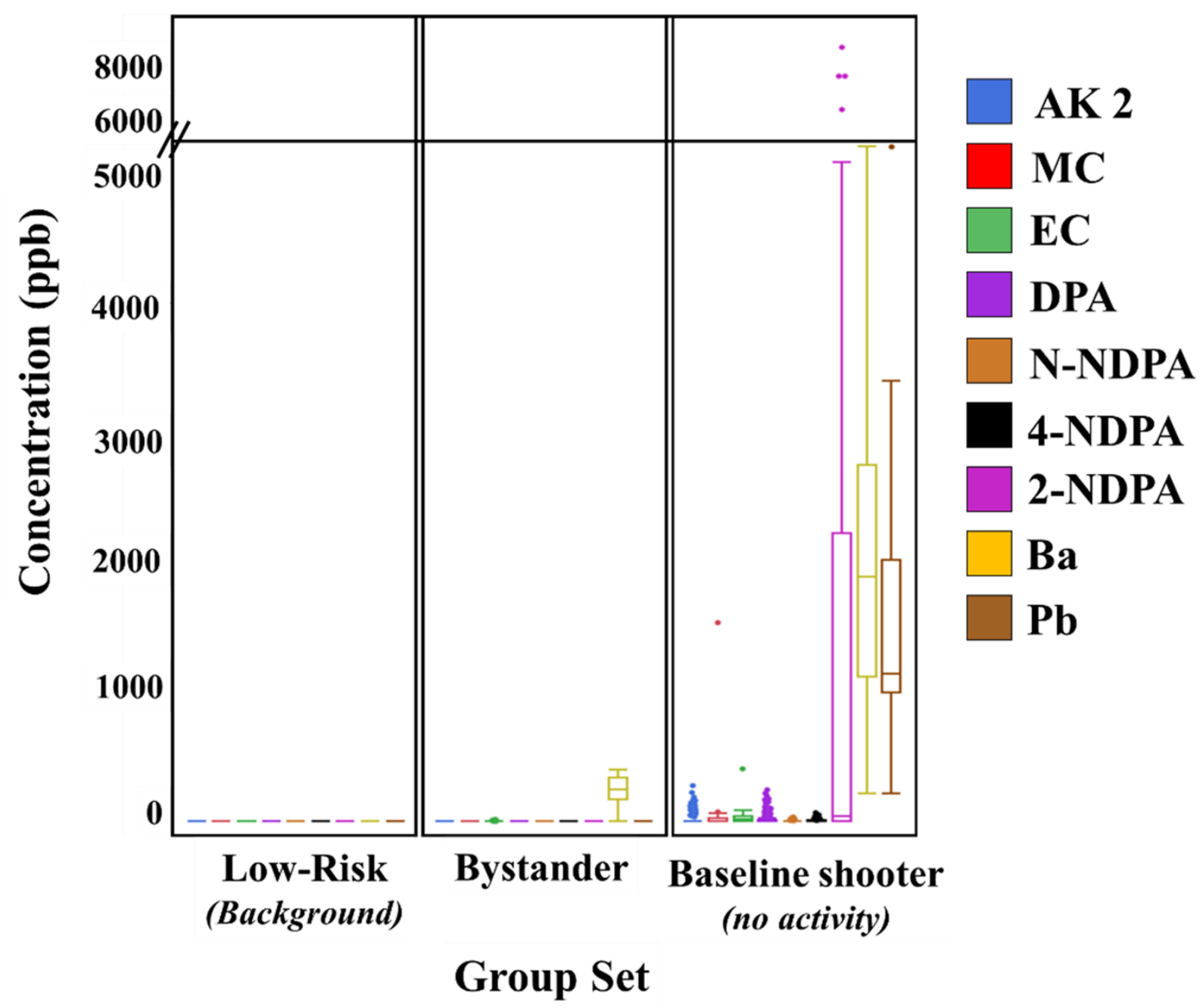

Figure 5.3: Boxplot comparisons between three populations. The low-risk (background samples), the bystander, and baseline shooter (no activity) responses for seven OGSR and two IGSR studied analytes are displayed.

Because of the environmental and safety regulations and the dimensions of the WVU ballistics range, the facility had a laminar flow system to direct air away from the shooter. This setup may have prevented analytes from reaching the bystanders and influenced their distribution. A second consideration for future studies is for bystanders to remain stationary for an extended period after a discharge event to allow for the potential settling of the constituents (i.e., one minute). In this study's experimental design, the shooter and the bystander left the range immediately after firing. One study by Luten et al. utilized a particle counter and observed that 
GSR can remain airborne several hours due to their size and dynamics [56]. Therefore, it is hypothesized that insufficient time was allowed for the GSR components to settle on the bystanders [56-58]. Hence, future experiments should be geared towards sedimentation rates of varying particle sizes and compounds. Nonetheless, the results illustrate the importance of considering environmental conditions, including airflows in indoor settings as well as time between discharge and collection for the interpretation of the data.

\subsubsection{Summary of exploratory analysis and frequency of occurrence of IGSR and OGSR}

The data was further categorized into two groups labeled as Known Shooter and Known Non-Shooter. The authentic shooters, activity, and bystander were grouped into the Known Shooter since we want to consider an association with a firearm-related incident, whereas the highand low-risk were placed in the Known Non-Shooter set. As shown in Figure 5.4, the known shooters produce higher IGSR and OGSR signal responses. Ultimately, the IGSR analytes for the high-risk and the bystander populations displayed higher signatures versus the low-risk population. The agricultural workers, police officers, and mechanics displayed residues with similar chemical compositions to the shooter set but at lower reported concentrations. The low-risk population set revealed less IGSR particulates than the high-risk and bystander sets and was comparable to the negative controls of the collection team. 

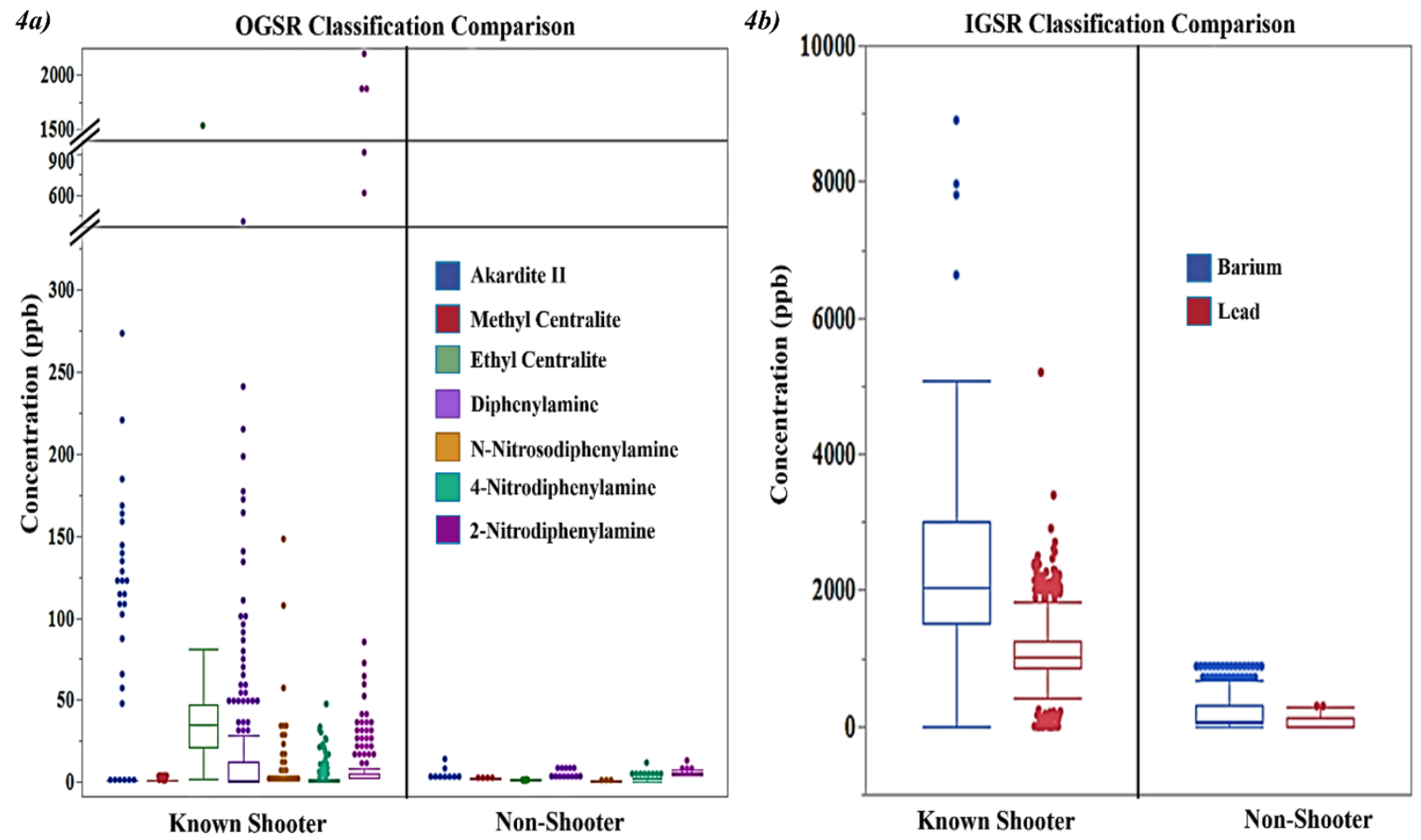

\section{Classification}

Figure 5.4: Figure 5.4a) OGSR population comparison among all analytes tested. Seven OGSR analytes were monitored, including akardite II (blue), methyl centralite (red), ethyl centralite (green), diphenylamine (dark purple), N-nitrosodiphenylamine (orange), 4-nitrodiphenylamine (turquoise), and 2-nitrodiphenylamine (magenta). Overall, the authentic shooting populations (shooter and activity) showed higher concentrations of the analytes versus the remaining populations (high- and low-risk and bystander). However, there were low concentration levels for both diphenylamine and its byproducts in the low- and high-risk populations. Figure 5.4b) IGSR population comparison among the populations tested. Ultimately two IGSR analytes were monitored, including barium (blue) and lead (red). Similarly, the authentic shooting populations (shooter and activity) showed higher concentrations of the analytes versus the remaining populations (high- and low-risk and bystander). However, there were comparable concentration levels for both IGSR and in the high-risk population.

Looking closer at Figure 5.4, there is some overlap in the IGSR signals for the Non-shooter set versus the Known Shooter populations which can lead to higher false positive rates if considered as the only signature for GSR identification. For the OGSR analytes, Known Shooter samples not only show more analytes present, but the response levels indicate minimal overlap with samples from the Non-Shooter population. However, the OGSR signals demonstrate a larger 
dispersion, especially for AK 2 and DPA. This could be the result of multiple factors including varying propellant composition across manufacturers used in this study, the volatile nature of the constituents, or simply the entropic nature of a deflagration event.

These findings indicate that it is not uncommon to find multiple OGSR analytes on the same sample. However, the combination of OGSR and IGSR provides a better indication of the presence of GSR. Most importantly, by using an IGSR/OGSR detection criterion, the identification of GSR (as potential false positives) decreased. Thus, further stressing the relevancy of using more comprehensive profiles for decision making.

The benefit of any exploratory method, like the one used in this study, is that data visualization permits a deeper understanding of the factors influencing the data distribution. On the other hand, the amount of time required and the difficulty to correctly evaluate and characterize each dataset can be troublesome based on the complexity of a sample. Additionally, it does not address a significant concern of GSR evidence, which centers around the probability of finding evidence given there was a or was not firing event - the two mutually exclusive hypotheses. Hence, techniques like machine learning algorithms can provide more comprehensive classifications when closely monitored and rigorously tested.

\subsubsection{Neural network machine learning for GSR identification}

Neural networks $(N N)$, also known as artificial neural networks (ANNs), are a subset of machine learning and are the heart of deep learning algorithms. Their name and structure are inspired by the human brain which mimic the signaling and communication of biological neurons [53]. Neural networks are comprised of node layers, including an input layer, one or more hidden layer(s), and output layers. For this study, the concentration levels for detected analytes were reported in parts-per-billion (ppb) for each population set. Neural networks can be created for 
different purposes and serve as both classification and prediction systems. Here, the predictive/supervised learning approach was implemented to 1) monitor the effectiveness and success rates of the method and 2) generate probabilistic outputs to calculate the likelihood ratios. The subpopulations were grouped further because the goal is to ultimately differentiate shooters from non-shooters using a single methodology. The two populations used for non-shooters were the low-risk and high-risk sample sets, whereas the authentic shooter comprised of the shooters (no-activity), bystanders, and post-shooting activity sample sets.

After exploratory analysis, the calculated concentrations of the five subpopulations were first preprocessed using min-max normalization. Each feature value i.e., analyte response, in each subpopulation was evaluated for its minimum and maximum values highlighted by Equation 5.2

$$
x_{i j} \Leftarrow \frac{x_{i j}-\min _{i}}{\max _{j}-\min _{j}}
$$

Equation 5.2

One can regard this as shifting all the observations so that the minimum value is zero, and then dividing by either the maximum of the shifted data, or equivalently the range of the original data. It also scaled all observations so that the minimum possible value is zero and the maximum is one. Min-max normalization is typical in neural network modeling and ensures that compound with comparatively large concentrations such as $\mathrm{Ba}$ and $\mathrm{EC}$ in this case do not control the network training. Rather, scaling ensures that the network training focuses on relative concentration differences rather that absolute values. The 0-1 scale is compatible with network node outputs that are also scaled as $0-1$.

After choosing the min-max normalization approach, those scaled values were then introduced to the model using a 60:20:20 training:validation:testing structure. Furthermore, to estimate the variability in the accuracy of the test, a 10-fold cross-validation was repeated 10 times 
on the combined data set. The performance of the scaled data was estimated for the NN's misclassification rates (Table S5.2) and by calculating the respective coefficient of variation (CV) which considers both the standard deviation $(\sigma)$ and the means $(\mu)$ to measure of dispersion of a probability distribution or frequency distribution, outlined by Equation 5.3

$$
C V=\frac{\sigma}{\mu}
$$

Equation 5.3

In this application, the $\mathrm{CV}$ values are a ratio of the variability in accuracy to the average accuracy. A high average accuracy coupled with a low CV is desirable as this indicates the method has performed well on many different training sets. Note that, as with all cross-validation methods, the picture will likely be more rosey than reality, but understanding and accounting for variability in prediction accuracy is preferrable to simply choosing the method with the highest accuracy without assessing its variability.

The method's accuracy demonstrated very low false-positive and false-negative rates (Table S5.2) for identifying the baseline shooters against the other subpopulations. Hence, an adaptive model that learns through vigorous trials such as neural networks is appropriate for analyzing newly acquired data. Moreover, the comparable accuracy within each subpopulations' training, testing and validation sets is a good indicator the model is not overfitting the data. The overall structure of the NN used in this study is outlined in Table S5.3.

\subsubsection{Likelihood ratios (LR) as probabilistic assessments of the weight of evidence}

Each subpopulation's probability outputs were calculated using a neural network model which allowed for the estimation of likelihood ratios. Although relatively small, the overall dataset in this study generated experimental observations that may closely resemble casework specimens and data. With that goal in mind, the low- and high-risk background samples express simple to complex situations, respectively. For the authentic shooting set, we wanted to mimic situations 
where analysts may encounter in firearm-related cases. The complexity of the shooter scenarios ranged from samples recovered immediately after a discharge event (simple) to situations where a suspect performed post-shooting activities, and even those near a shooting event (complex). For the latter two, the expected challenges were high analyte loss (represented by the post-shooting activities set) and increased difficulty when differentiating from shooters (represented by the bystander set). In this study, the $H_{1}$ and $H_{2}$ hypotheses at the source level are defined as:

$H_{1}$ : The trace originated from a discharged firearm (and hence, are GSR)

$\mathrm{H}_{2}$ : The trace originated from a non-firearm source (i.e., other environmental source, and hence are not GSR)

For purposes of the hypotheses, the low-risk background samples were considered as the Ground Truth for non-GSR cases, while the Baseline shooter (no activity) set was considered the Ground Truth for GSR-containing samples. To evaluate the performance of the LR, we examined how often the LR was $>1$ (or $\log$ LR > 0) when samples were anticipated to have GSR present. Contrarily, we also monitored how often the LR was $<1$ (or $\log$ LR $<0$ ) when true non-GSR datasets showed an absence of GSR. Those observations are displayed as histograms that depict the frequency when $H_{1}$ or $H_{2}$ is true (y-axis) versus the log LR ( $x$-axis). These histograms can further assess the performance of the LR.

The discriminating power of this methodology can distinguish cases where $H_{1}$ and $H_{2}$ are respectively true. For instance, the greater the separation between a subpopulation's histogram, the better the discriminating power, which increases the confidence of a given sample. Similarly, if one subpopulation's resulting histogram overlaps another histogram, the greater the misleading evidence rates, which also lowers the confidence. Therefore, the larger the LR, the evidence is more likely (i.e., stronger support) if the trace originated from GSR than other sources. 
An alternative strategy for assessing the performance of the likelihood ratios is using Tippet plots. A Tippet plot is a plot of two empirical "complementary" cumulative distribution functions (ECCDFs). That is, a ECCDF is an estimate of a complementary cumulative distribution function and given a sample of data (of size $n$ ) is computed by

$$
\hat{F}(x)=\frac{1}{n} \sum_{i=1}^{n} I\left[x_{i} \geq x\right]
$$

where $I\left[x_{i} \geq x\right]$ is an indicator function that takes the value one if $x_{i} \geq x$, and zero otherwise.

A Tippet plot is a plot of these functions versus $x$ where $x$ is the $L R$ computed under $H_{1}$ and $H_{2}$ - that is there are two ECCDFs in a Tippet plot [59]. Tippet plots can graphically display discriminating power by consideration of the vertical separation of true- $H_{1}$ and true- $H_{2}$ curves at a given value of $\log \mathrm{LR}$ axis. The more separated at a given LR, the higher discrimination at that value. Additionally, the rates of misleading evidence (RoMEs) can be visually estimated by considering the area above (or under) the curves to left (or right) of the $\operatorname{line} \log _{10}(L R)=0$, depending on whether $H_{1}$ and $H_{2}$ is of interest [59]. For instance, when $H_{1}$ and $H_{2}$ are true ( $\log _{10}$ $\mathrm{LR}>0$ and $\log _{10} \mathrm{LR}<0$, respectively), the $\mathrm{H}_{2}$ curve can help visualize false positives (i.e., $\log _{10}$ LR > 0 when no GSR should be present). Oppositely, the misleading rates for the $H_{l}$ curve represent false negatives and are estimated when $\log _{10} \mathrm{LR}<0$ (i.e., when GSR should be present).

\subsubsection{Baseline shooters (no activity) versus Low-Risk Background likelihood ratios}

The first assessment of the LR was conducted using the two subpopulations of low-risk (known non-shooters) and the baseline shooter (no activity). For this case, we expected minimal to no overlap when OGSR and IGSR information was combined. Figure $\mathbf{5 . 5}$ shows a clear separation between baseline shooter (no activity) $\left(H_{l}\right)$ and low-risk $\left(H_{2}\right)$ histograms. 

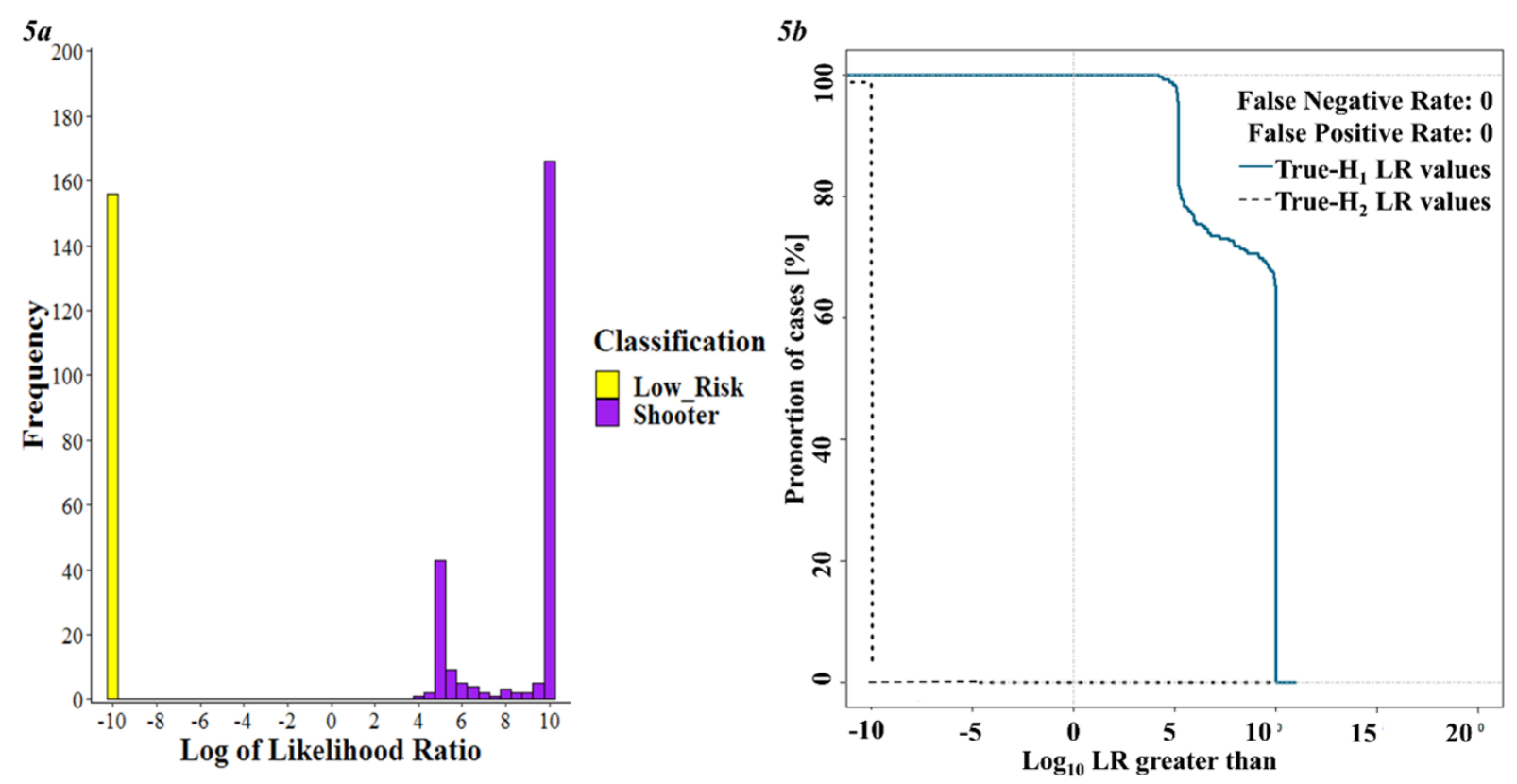

Figure 5.5: Figure 5.5a) Histograms of Baseline shooter (no activity) $\left(\mathrm{H}_{1}\right)$ and low-risk $\left(\mathrm{H}_{2}\right)$ subpopulation. The Baseline shooter displays a broader spread due to varying concentration levels of OGSR and IGSR signals. The low-risk subpopulation has a condensed range and is centralized at -10. Figure 5.5b) Tippet plot illustrating no instances of either false positives or false negatives.

Additionally, the Tippet plots reflect the highest discriminating ability to differentiate between the subpopulations with minimal RoME. This result is similar to the observation during the initial exploratory analysis. Because of this optimum distinction, these subpopulations were regarded as the lower and upper bounds for further comparisons.

\subsubsection{High-Risk likelihood ratios}

After the Baseline shooter and low-risk populations bounds were established, the remaining samples, starting with the high-risk set $\left(H_{2}\right)$, baseline shooter $\left(H_{1}\right)$, and the low-risk sets $\left(H_{2}\right)$, were tested against them to monitor the rates of misleading evidence in more complex situations. The Figure 5.6a histogram illustrates there was a separation between all three populations, with larger dispersion of $\operatorname{logLRs}$ of the high-risk set towards less negative values as compared to the low-risks, which is due to the relative presence of EC in some of the samples as well as IGSR elements in this subpopulation. Nonetheless, the profiles observed in the high-risk 
set were easily distinguished from the shooter set by the NN. As a result, the Tippet plot in Figure

5.6b shows low rates of misleading evidence even for samples with high contamination risk.
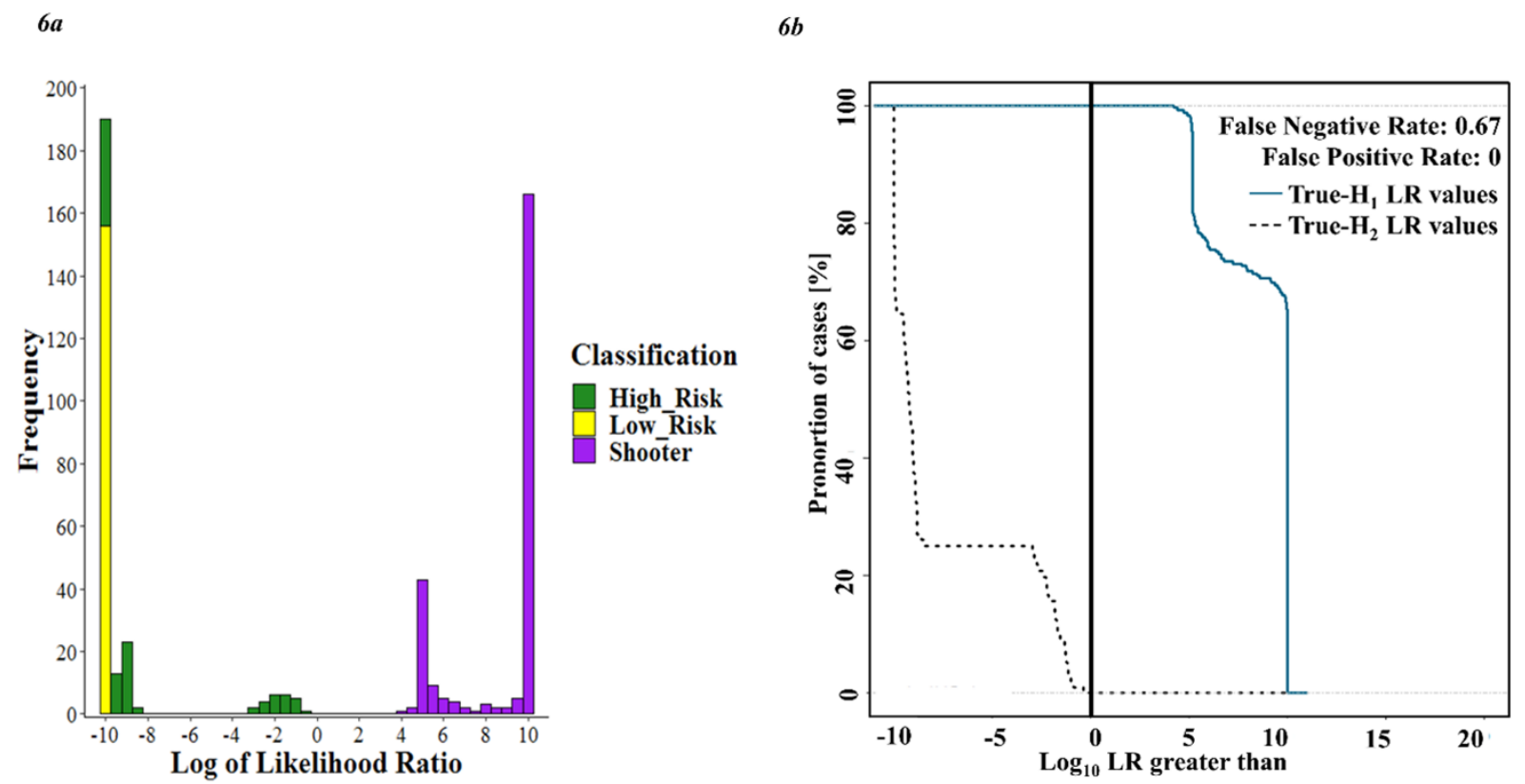

Figure 5.6: Figure 5.6a) Histogram comparison of Baseline shooter (no activity), low-risk, and high-risk subpopulations. The high-risk population displays a broader spread for its histogram range due to varying concentration levels of the analytes. Figure 5.6b) Tippet plot illustrating the RoME compared to the Baseline shooter (no activity).

\subsubsection{Post-shooting activity likelihood ratios}

This approach continued to investigate the remaining subpopulations to evaluate and attempt to discriminate between low-risk background $\left(H_{2}\right)$ versus post-shooting activity set $\left(H_{1}\right)$ samples where some sample loss is expected. Figure 5.7a shows some activity samples presenting $\log$ LR lower than zero. Although no false positives are observed (Figure 5.7b), some false negatives are present due to the loss of GSR traces (RoME 1.28\%). Interestingly, there is notable overlap with the baseline shooter sample set. This overlap is a result of this subpopulation possessing OGSR and IGSR signals in a single sample. As expected, the log LR of the postshooting activity set were spread out towards lower values than the shooter sets, However, the data 
still indicates that LR can be used to assess the weight of evidence, with strong support for GSR presence observed in samples that have gone through everyday activities such as rubbing, cleaning with hand sanitizer, or running outdoors.
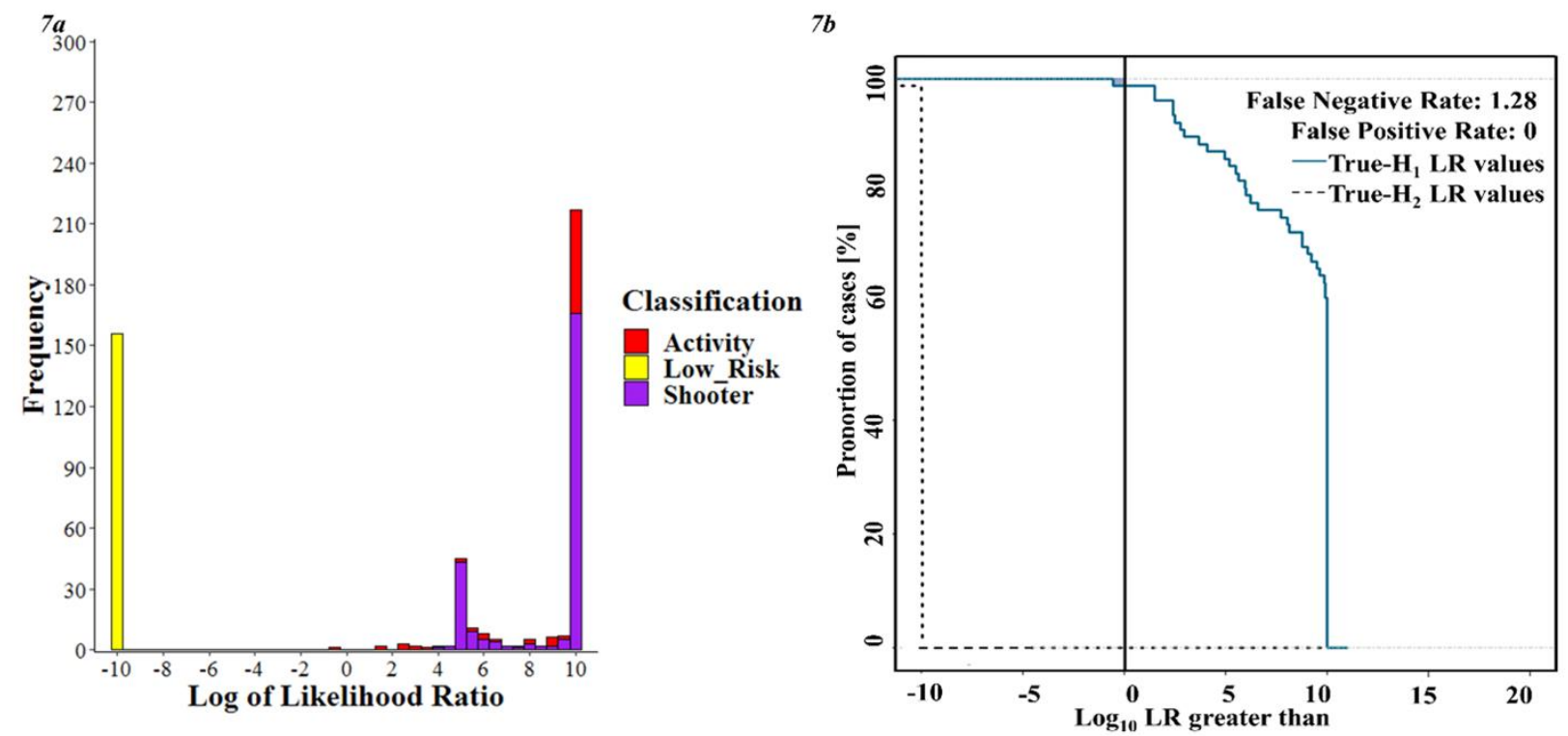

Figure 5.7: Figure 5.7a) Histogram comparison of Baseline shooter (no activity), low-risk, and post-shooting activity subpopulations. The post-shooting activity population displays a broader spread for its histogram range due to varying concentration levels of the analytes with heavy overlap with the Baseline shooter (no activity) samples. Figure 5.7b) Tippet plot illustrating the negatives compared to the low-risk background.

\subsubsection{Bystander likelihood ratios}

Finally, the bystanders (considered as the subpopulation set for $H_{1}$ ) were compared and were separated from the baseline shooter sample set $\left(H_{1}\right)$ represented in Figure 5.8. Additionally, there is no overlap between the bystander set and the low-risk sets which is displayed by the Tippet plots having low RoMEs. These observations can be explained based on the general substantial decrease of the concentrations of both OGSR and IGSR in the bystander's stubs. Significant relative differences of inorganic elements and organic compounds were observed between individuals who fired the gun and those standing a distance away from a discharge event, except for persistence of $\mathrm{Ba}$ and $\mathrm{EC}$. 
Caution should be taken in interpreting results from this subpopulation as we learned from exploratory data analysis that there might be uncontrolled factors in our experiments biasing the results. Future studies will address this issue by controlling experimental factors that would mimic different environmental conditions and deposition settling times for individuals in the vicinity of the firing event. As a result, the findings on this subpopulation cannot be generalized at this point. Nonetheless, it highlights important aspects that may play a role on GSR transfer to bystanders when assessing casework data, such as airflow conditions, settling times, and time between discharge and collection.
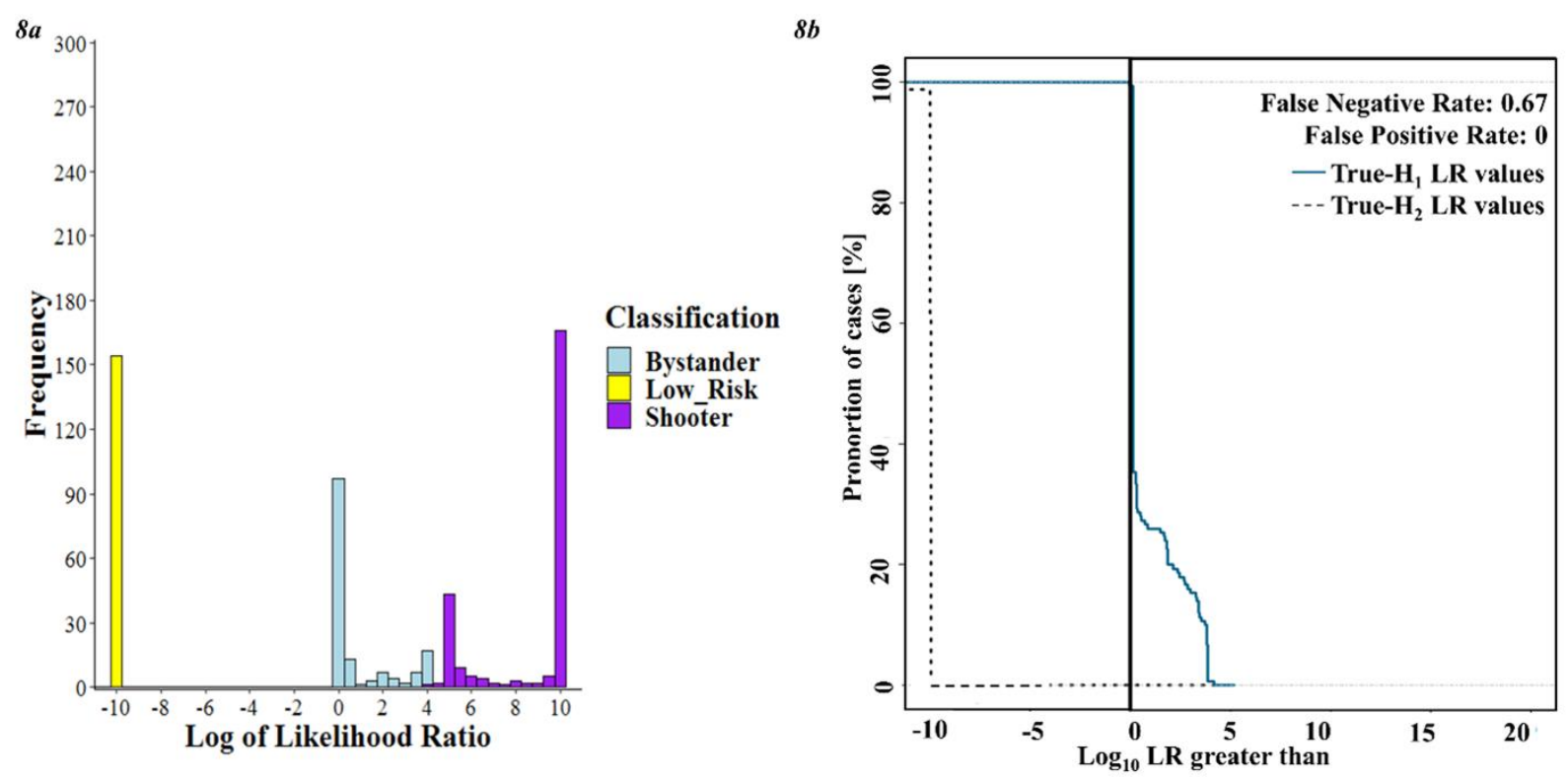

Figure 5.8: Figure 5.8a) Histogram comparison of Baseline shooter (no activity), low-risk, and Bystander subpopulations. The Bystander population displays a smaller spread for its histogram range due to varying concentration levels of the analytes. Figure 5.8b) Tippet plot illustrating the negatives compared to the low-risk background.

\subsection{Conclusions}

The LC-MS/MS is a highly versatile technique found in many crime laboratories, which is primarily used to analyze controlled substances (such as illegal narcotics), explosives, or toxicology samples. Our research has demonstrated that the applications of this instrument can be 
expanded to GSR, with dual identification of OGSR and IGSR through simple extractions and host-guest chemistry [17]. Additionally, it can be a reliable confirmatory method with relatively quick turnaround times and automation.

This study demonstrated that GSR evidence can be analyzed and classified accurately using machine learning algorithms and likelihood ratios with low error rates. The observation and investigations into the RoME values (rates of false-negatives and false positives) presented in this work is an additional step into a deeper understanding of GSR behavior. Additionally, machine learning results can generate likelihood ratios for subpopulations with reasonable accuracies and differentiation (Figure 5.9).

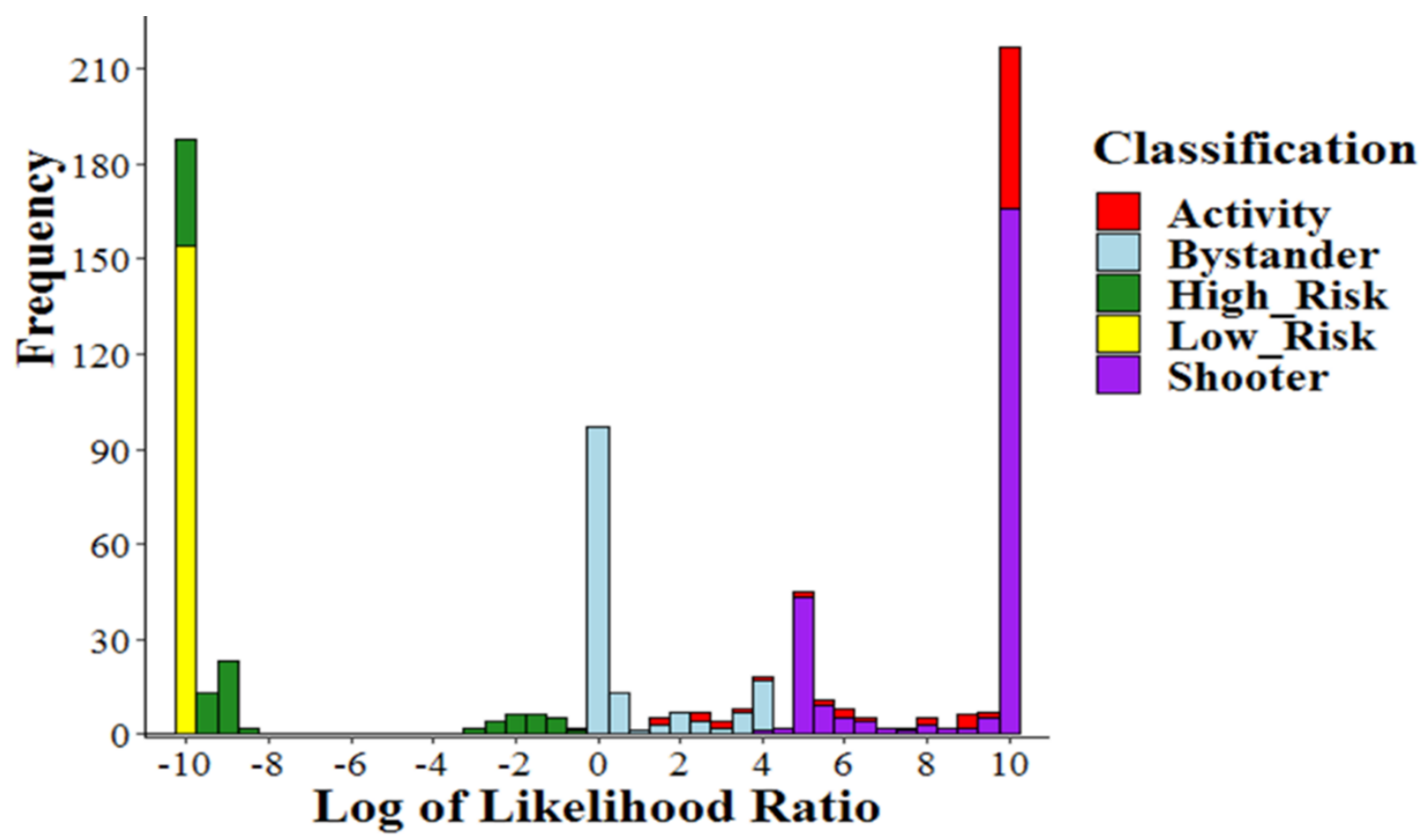

Figure 9: Neural Network structure to initially train and validate the authentic and low-risk populations. The nine analytes' spectra were inputted and processed by each node. Each compound tested was preprocessed based on criteria focusing on the peak response. Those considerations included a signal-to-noise above three and having an intensity above the limits of detection and quantitation. 
These preliminary results highlight the potential to distinguish complex groups such as bystanders and high-risk individuals (to a degree) versus low-risk and baseline shooter populations. Also, it shows that identification of GSR is possible even after a shooter engages in some typical post-shooting activities. In terms of the bystander and post-shooting activity populations, the overlap between those populations was not surprising. This is due to the transfer of GSR components near a firing event. The region of overlap is the greatest risk of false positives (in the case of bystanders) or false negatives (shooter with post-shooting activity). More importantly, there is a clear distinction between high-risk backgrounds (non-shooters) and authentic shooters. This was possible because combining IGSR and OGSR information yields higher confidence in classifications and provides enhanced evidentiary value to GSR evidence. Thus, researchers and practitioners would benefit from 1) implementing techniques that can perform IGSR/OGSR analysis and 2) evaluating the weight of the evidence as part of the data interpretation.

This study demonstrates the potential of using IGSR/OGSR combined profiles and machine learning algorithms to estimate likelihood ratios as a more comprehensive framework to assess the weight of firearms discharge the evidence. More extensive studies and collaboration efforts should be one objective for the forensic research community and additional bystander studies to monitor the underlying mechanism associated with transfer and persistence and further apply the LR at the activity level. Notably, a machine learning model's probabilistic data assessment may open new avenues for more comprehensive interpretative frameworks for GSR evidence. 


\section{Author Contributions}

The manuscript was written through the contributions of all authors. All authors have given approval to the final version of the manuscript.

\section{Conflicts of interest}

The authors declare that they have no known competing financial interests or personal relationships that could have appeared to influence the work reported in this paper.

\section{Acknowledgments}

We want to acknowledge Dr. Keith Morris and James Hamilton for collecting authentic samples and accessing the WVU ballistics laboratory. We also want to acknowledge Kourtney Dalzell, Colby Ott, and Courtney Vander Pyl to collect authentic samples from all populations. Additionally, the authors thank Meghan Prusinowski for her assistance in programming.

This project is sponsored by Award No. 2018-DU-BX-0186 and 2019-R2-CX-044 from the National Institute of Justice to West Virginia University. The opinions, findings, and conclusions are those of the authors and do not necessarily reflect those of the Department of Justice. 


\subsection{References for Chapter 5}

[1] M.D. Gallidabino, L.P. Barron, C. Weyermann, F.S. Romolo, Quantitative profile-profile relationship (QPPR) modelling: A novel machine learning approach to predict and associate chemical characteristics of unspent ammunition from gunshot residue (GSR), Analyst. 144 (2019) 1128-1139. https://doi.org/10.1039/c8an01841c.

[2] M. Maitre, K.P. Kirkbride, M. Horder, C. Roux, A. Beavis, Current perspectives in the interpretation of gunshot residues in forensic science: A review, Forensic Sci. Int. 270 (2017) 111. https://doi.org/10.1016/j.forsciint.2016.09.003.

[3] C. Hofstetter, M. Maitre, A. Beavis, C.P. Roux, C. Weyermann, A.L. Gassner, A study of transfer and prevalence of organic gunshot residues, Forensic Sci. Int. 277 (2017) 241-251. https://doi.org/10.1016/j.forsciint.2017.06.013.

[4] L.S. Blakey, G.P. Sharples, K. Chana, J.W. Birkett, Fate and Behavior of Gunshot Residue-A Review, J. Forensic Sci. 63 (2018) 9-19. https://doi.org/10.1111/1556-4029.13555.

[5] W. Feeney, C. Vander Pyl, S. Bell, T. Trejos, Trends in composition, collection, persistence, and analysis of IGSR and OGSR: A review, Forensic Chem. 19 (2020) 100250. https://doi.org/10.1016/j.forc.2020.100250.

[6] J. Moran, S. Bell, Analysis of organic gunshot residue permeation through a model skin membrane using ion mobility spectrometry, Int. J. Ion Mobil. Spectrom. 16 (2013) 247-258. https://doi.org/10.1007/s12127-013-0138-0.

[7] J. French, R. Morgan, J. Davy, The secondary transfer of gunshot residue: an experimental investigation carried out with SEM-EDX analysis, X-Ray Spectrom. 43 (2014) 5661. https://doi.org/10.1002/xrs.2498. 
[8] J.C. French, R.M. Morgan, P. Baxendell, P.A. Bull, Multiple transfers of particulates and their dissemination within contact networks, Sci. Justice. 52 (2012) 33-41. https://doi.org/10.1016/j.scijus.2011.05.001.

[9] S. Practice, S. Handbook, S. Practice, Standard Practice for Gunshot Residue Analysis by Scanning Electron Microscopy/Energy Dispersive X-Ray Spectrometry, ASTM Int. i (2020) 1-5. https://doi.org/10.1520/E1588-20.

[10] K. Menking-Hoggatt, C. Martinez, C. Vander, E. Heller, E. Chip, L. Arroyo, T. Trejos, C. Vander Pyl, E. Heller, E. “Chip” Pollock, L. Arroyo, T. Trejos, Development of tailor-made inorganic gunshot residue (IGSR) microparticle standards and characterization with a multitechnique approach, Talanta. 225 (2021) 121984. https://doi.org/10.1016/j.talanta.2020.121984. [11] Z. Abrego, A. Ugarte, N. Unceta, A. Fernández-Isla, M.A. Goicolea, R.J. Barrio, Unambiguous characterization of gunshot residue particles using scanning laser ablation and inductively coupled plasma-mass spectrometry, Anal. Chem. 84 (2012) 2402-2409. https://doi.org/10.1021/ac203155r.

[12] K. Menking-Hoggatt, L. Arroyo, J. Curran, T. Trejos, Novel LIBS method for microspatial chemical analysis of inorganic gunshot residues, J. Chemom. 35 (2021) 1-13. https://doi.org/10.1002/cem.3208.

[13] E. Goudsmits, G.P. Sharples, J.W. Birkett, Recent trends in organic gunshot residue analysis, Trac-Trends Anal. Chem. 74 (2015) 46-57. https://doi.org/10.1016/j.trac.2015.05.010. [14] B. Stevens, S. Bell, K. Adams, Initial evaluation of inlet thermal desorption GC-MS analysis for organic gunshot residue collected from the hands of known shooters, Forensic Chem. 2 (2016) 55-62. https://doi.org/10.1016/j.forc.2016.10.001. 
[15] R.V. Taudte, C. Roux, D. Bishop, L. Blanes, P. Doble, A. Beavis, Development of a UHPLC method for the detection of organic gunshot residues using artificial neural networks, Anal. Methods. 7 (2015) 7447-7454. https://doi.org/10.1039/c5ay00306g.

[16] C. Bonnar, R. Popelka-Filcoff, K.P. Kirkbride, Armed with the Facts: A Method for the Analysis of Smokeless Powders by Ambient Mass Spectrometry, J. Am. Soc. Mass Spectrom. 31 (2020) 1943-1956. https://doi.org/10.1021/jasms.0c00193.

[17] W. Feeney, K. Menking-Hoggatt, C. Vander Pyl, C.E. Ott, S. Bell, L. Arroyo, T. Trejos, Detection of Organic and Inorganic Gunshot Residues from Hands using Complexing Agents and LC-MS/MS, Anal. Methods. (2021). https://doi.org/10.1039/d1ay00778e.

[18] S.R. Khandasammy, A. Rzhevskii, I.K. Lednev, A Novel Two-Step Method for the Detection of Organic Gunshot Residue for Forensic Purposes: Fast Fluorescence Imaging Followed by Raman Microspectroscopic Identification, Anal. Chem. 91 (2019) 11731-11737. https://doi.org/10.1021/acs.analchem.9b02306.

[19] C. Bonnar, E.C. Moule, N. Lucas, K.E. Seyfang, R.P. Dunsmore, R.S. Popelka-Filcoff, K. Redman, K. Paul Kirkbride, Tandem detection of organic and inorganic gunshot residues using LC-MS and SEM-EDS, Forensic Sci. Int. 314 (2020) 110389. https://doi.org/10.1016/j.forsciint.2020.110389.

[20] V. Redouté Minzière, D. Werner, D. Schneider, M. Manganelli, B. Jung, C. Weyermann, A.L. Gassner, Combined Collection and Analysis of Inorganic and Organic Gunshot Residues, J. Forensic Sci. (2020). https://doi.org/10.1111/1556-4029.14314.

[21] Z. Abrego, N. Grijalba, N. Unceta, M. Maguregui, A. Sanchez, A. Fernandez-Isla, M.A. Goicolea, R.J. Barrio, A. Fernández-Isla, M.A. Goicolea, R.J. Barrió, A. Fernandez-Isla, M.A. Goicolea, R.J. Barrio, A novel method for the identification of inorganic and organic gunshot 
residue particles of lead-free ammunitions from the hands of shooters using scanning laser ablation-ICPMS and Raman micro-spectroscopy, Analyst. 139 (2014) 6232-6241. https://doi.org/10.1039/c4an01051e.

[22] J. Bueno, I.K. Lednev, Attenuated Total Reflectance-FT-IR Imaging for Rapid and Automated Detection of Gunshot Residue, Anal. Chem. 86 (2014) 3389-3396. https://doi.org/10.1021/ac4036718.

[23] C.E. Ott, K.A. Dalzell, P.J. Calderón-Arce, A.L. Alvarado-Gámez, T. Trejos, L.E. Arroyo, Evaluation of the Simultaneous Analysis of Organic and Inorganic Gunshot Residues Within a Large Population Data Set Using Electrochemical Sensors*, $\uparrow$, J. Forensic Sci. 65 (2020) 1935-1944. https://doi.org/10.1111/1556-4029.14548.

[24] L. Ali, K. Brown, H. Castellano, S.J. Wetzel, A Study of the Presence of Gunshot Residue in Pittsburgh Police Stations using SEM/EDS and LC-MS/MS, J. Forensic Sci. 61 (2016) 928-938. https://doi.org/10.1111/1556-4029.13077.

[25] A. Tarifa, J.R. Almirall, Fast detection and characterization of organic and inorganic gunshot residues on the hands of suspects by CMV-GC-MS and LIBS, Sci. Justice. 55 (2015) 168-175. https://doi.org/10.1016/j.scijus.2015.02.003.

[26] T. Trejos, C. Vander Pyl, K. Menking-Hoggatt, A.L. Alvarado, L.E. Arroyo, C. V Pyl, K. Menking-Hoggatt, A.L. Alvarado, L.E. Arroyo, Fast identification of inorganic and organic gunshot residues by LIBS and electrochemical methods, Forensic Chem. 8 (2018) 146-156. https://doi.org/10.1016/j.forc.2018.02.006.

[27] Z. Brozek-Mucha, On the prevalence of gunshot residue in selected populations - An empirical study performed with SEM-EDX analysis, Forensic Sci. Int. 237 (2014) 46-52. https://doi.org/10.1016/j.forsciint.2014.01.020. 
[28] S. Bell, L. Seitzinger, From binary presumptive assays to probabilistic assessments:

Differentiation of shooters from non-shooters using IMS, OGSR, neural networks, and likelihood ratios, Forensic Sci. Int. 263 (2016) 176-185. https://doi.org/10.1016/j.forsciint.2016.04.020.

[29] N. Lucas, H. Brown, M. Cook, K. Redman, T. Condon, H. Wrobel, K.P. Kirkbride, H. Kobus, A study into the distribution of gunshot residue particles in the random population, Forensic Sci. Int. 262 (2016) 150-155. https://doi.org/10.1016/j.forsciint.2016.02.050.

[30] Z. Brozek-Mucha, Chemical and morphological study of gunshot residue persisting on the shooter by means of scanning electron microscopy and energy dispersive X-ray spectrometry, Microsc. Microanal. 17 (2011) 972-982. https://doi.org/10.1017/S1431927611012141.

[31] R.A. Costa, L.C. Motta, C.A. Destefani, R.R.T. Rodrigues, K.S.D. Santo, G. Aquije, R. Boldrini, G.P.B. Athayde, M. Carneiro, W. Romao, Gunshot residues (GSR) analysis of clean range ammunition using SEM/EDX, colorimetric test and ICP-MS: A comparative approach between the analytical techniques, Microchem. J. 129 (2016) 339-347. https://doi.org/10.1016/j.microc.2016.07.017.

[32] A. Zeichner, B. Eldar, B. Glattstein, A. Koffman, T. Tamiri, D. Muller, Vacuum collection of gunpowder residues from clothing worn by shooting suspects, and their analysis by GC/TEA, IMS, and GC/MS, J. Forensic Sci. 48 (2003) 961-972.

https://doi.org/10.1520/jfs2002390.

[33] M. Cook, Gunshot residue contamination of the hands of police officers following startof-shift handling of their firearm, Forensic Sci. Int. 269 (2016) 56-62. https://doi.org/10.1016/j.forsciint.2016.11.002. 
[34] R. V. Gerard, E. Lindsay, M.J. McVicar, E.D. Randall, A. Gapinska, Observations of gunshot residue associated with police officers, their equipment, and their vehicles, J. Can. Soc. Forensic Sci. 45 (2012) 57-63. https://doi.org/10.1080/00085030.2012.10757183.

[35] S. Charles, N. Geusens, E. Vergalito, B. Nys, Interpol review of gunshot residue 20162019, Forensic Sci. Int. Synerg. 2 (2020) 416-428. https://doi.org/10.1016/j.fsisyn.2020.01.011.

[36] A. Stamouli, L. Niewöhner, M. Larsson, B. Colson, S. Uhlig, L. Fojtasek, F. Machado, L. Gunaratnam, Survey of gunshot residue prevalence on the hands of individuals from various population groups in and outside Europe, Forensic Chem. 23 (2021) 1-8. https://doi.org/10.1016/j.forc.2021.100308.

[37] W. Tucker, N. Lucas, K.E. Seyfang, K.P. Kirkbride, R.S. Popelka-Filcoff, Gunshot residue and brakepads: Compositional and morphological considerations for forensic casework, Forensic Sci. Int. 270 (2017) 76-82. https://doi.org/10.1016/j.forsciint.2016.11.024.

[38] Ç. Aksoy, T. Bora, N. Şenocak, F. Aydın, F. Aydin, A new method to reduce false positives due to antimony in detection of gunshot residues, Forensic Sci. Int. 250 (2015) 87-90. https://doi.org/10.1016/j.forsciint.2015.03.006.

[39] G.M. Ingo, M. D’Uffizi, G. Falso, G. Bultrini, G. Padeletti, Thermal and microchemical investigation of automotive brake pad wear residues, Thermochim. Acta. 418 (2004) 61-68. https://doi.org/10.1016/j.tca.2003.11.042.

[40] Z.P. Wu, Y. Tong, J.Y. Yu, X.R. Zhang, C.X. Pan, X.Y. Deng, Y.C. Xu, Y.X. Wen, Detection of N,N'-diphenyl-N,N'-dimethylurea (methyl centralite) in gunshot residues using MS-MS method, Analyst. 124 (1999) 1563-1567. https://doi.org/10.1039/a904917g.

[41] Y. Tong, Z. Wu, C.D. Yang, J.Y. Yu, X.R. Zhang, S.J. Yang, X.Y. Deng, Y.C. Xu, Y.X. Wen, Z.P. Wei, C.D. Yang, J.Y. Yu, X.R. Zhang, S.J. Yang, X.Y. Deng, Y.C. Xu, Y.X. Wen, 
Determination of diphenylamine stabilizer and its nitrated derivatives in smokeless gunpowder using a tandem MS method, Analyst. 126 (2001) 480-484. https://doi.org/10.1039/b0101830. [42] Z. Brożek-Mucha, A study of gunshot residue distribution for close-range shots with a silenced gun using optical and scanning electron microscopy, X-ray microanalysis and infrared spectroscopy, Sci. Justice. 57 (2017) 87-94. https://doi.org/10.1016/j.scijus.2016.11.004.

[43] M. Manganelli, C. Weyermann, A.L. Gassner, Surveys of organic gunshot residue prevalence: Comparison between civilian and police populations, Forensic Sci. Int. 298 (2019) 48-57. https://doi.org/10.1016/j.forsciint.2019.02.050.

[44] M. Maitre, S. Chadwick, K.P. Kirkbride, A.L. Gassner, C. Weyermann, A. Beavis, C. Roux, An investigation on the secondary transfer of organic gunshot residues, Sci. Justice. 59 (2019) 248-255. https://doi.org/10.1016/j.scijus.2019.01.007.

[45] A. Biedermann, S. Bozza, F. Taroni, Probabilistic evidential assessment of gunshot residue particle evidence (Part I): Likelihood ratio calculation and case pre-assessment using Bayesian networks, Forensic Sci. Int. 191 (2009) 24-35.

https://doi.org/10.1016/j.forsciint.2009.06.004.

[46] F.S. Romolo, P. Margot, Identification of gunshot residue: a critical review, Forensic Sci. Int. 119 (2001) 195-211.

[47] B. Cardinetti, C. Ciampini, S. Abate, C. Marchetti, F. Ferrari, D. Di Tullio, C. D’Onofrio, G. Orlando, L. Gravina, L. Torresi, G. Saporita, A proposal for statistical evaluation of the detection of gunshot residues on a suspect, Scanning. 28 (2006) 142-147. https://doi.org/10.1002/sca.4950280302. 
[48] N.K. Damary, M. MandelY, N. Levin, E. Izraeli, Calculation of likelihood ratios for gunshot residue evidence-statistical aspects, Law, Probab. Risk. 15 (2016) 107-125. https://doi.org/10.1093/lpr/mgw001.

[49] T.J. Hannigan, S.D. McDermott, C.M. Greaney, J. O’Shaughnessy, C.M. O’Brien, Evaluation of gunshot residue (GSR) evidence: Surveys of prevalence of GSR on clothing and frequency of residue types, Forensic Sci. Int. 257 (2015) 177-181.

https://doi.org/10.1016/j.forsciint.2015.08.003.

[50] A. Bolck, A. Stamouli, Likelihood Ratios for categorical evidence; Comparison of LR models applied to gunshot residue data, Law, Probab. Risk. 16 (2017) 71-90. https://doi.org/10.1093/lpr/mgx005.

[51] A. Biedermann, S. Bozza, F. Taroni, Probabilistic evidential assessment of gunshot residue particle evidence (Part II): Bayesian parameter estimation for experimental count data, Forensic Sci. Int. 206 (2011) 103-110. https://doi.org/10.1016/j.forsciint.2010.07.009.

[52] L.S. Blakey, G.P. Sharples, K. Chana, J.W. Birkett, Fate and Behavior of Gunshot Residue-A Review, J. Forensic Sci. 63 (2018) 9-19. https://doi.org/10.1111/1556-4029.13555. [53] C.C. Aggarwal, Neural Networks and Deep Learning, Springer, 2018. https://doi.org/10.1201/b22400-15.

[54] R. Cook, I.W. Evett, G. Jackson, P.J. Jones, J.A. Lambert, A hierarchy of propositions: Deciding which level to address in casework, Sci. Justice - J. Forensic Sci. Soc. 38 (1998) 231239. https://doi.org/10.1016/S1355-0306(98)72117-3.

[55] B. Yeager, K. Bustin, J. Stewart, R. Dross, S. Bell, Evaluation and validation of ion mobility spectrometry for presumptive testing targeting the organic constituents of firearms discharge residue, Anal. Methods. 7 (2015) 9683-9691. https://doi.org/10.1039/c5ay02417j. 
[56] R. Luten, D. Neimke, M. Barth, L. Niewoehner, Investigating airborne GSR particles by the application of impactor technology, Forensic Chem. 8 (2018) 72-81. https://doi.org/10.1016/j.forc.2018.02.005.

[57] L. Fojtášek, T. Kmječ, Time periods of GSR particles deposition after discharge-final results, Forensic Sci. Int. 153 (2005) 132-135. https://doi.org/10.1016/j.forsciint.2004.09.127. [58] E. Diaz, J.E. Souza Sarkis, S. Viebig, P. Saldiva, Measurement of airborne gunshot particles in a ballistics laboratory by sector field inductively coupled plasma mass spectrometry, Forensic Sci. Int. 214 (2012) 44-47. https://doi.org/10.1016/j.forsciint.2011.07.016.

[59] G. Zadora, A. Martyna, D. Ramos, C. Aitken, Statistical Analysis in Forensic Science: Evidential Values of Multivariate Physicochemical Data, 2014.

https://doi.org/10.1002/9781118763155. 


\section{CHAPTER 6}

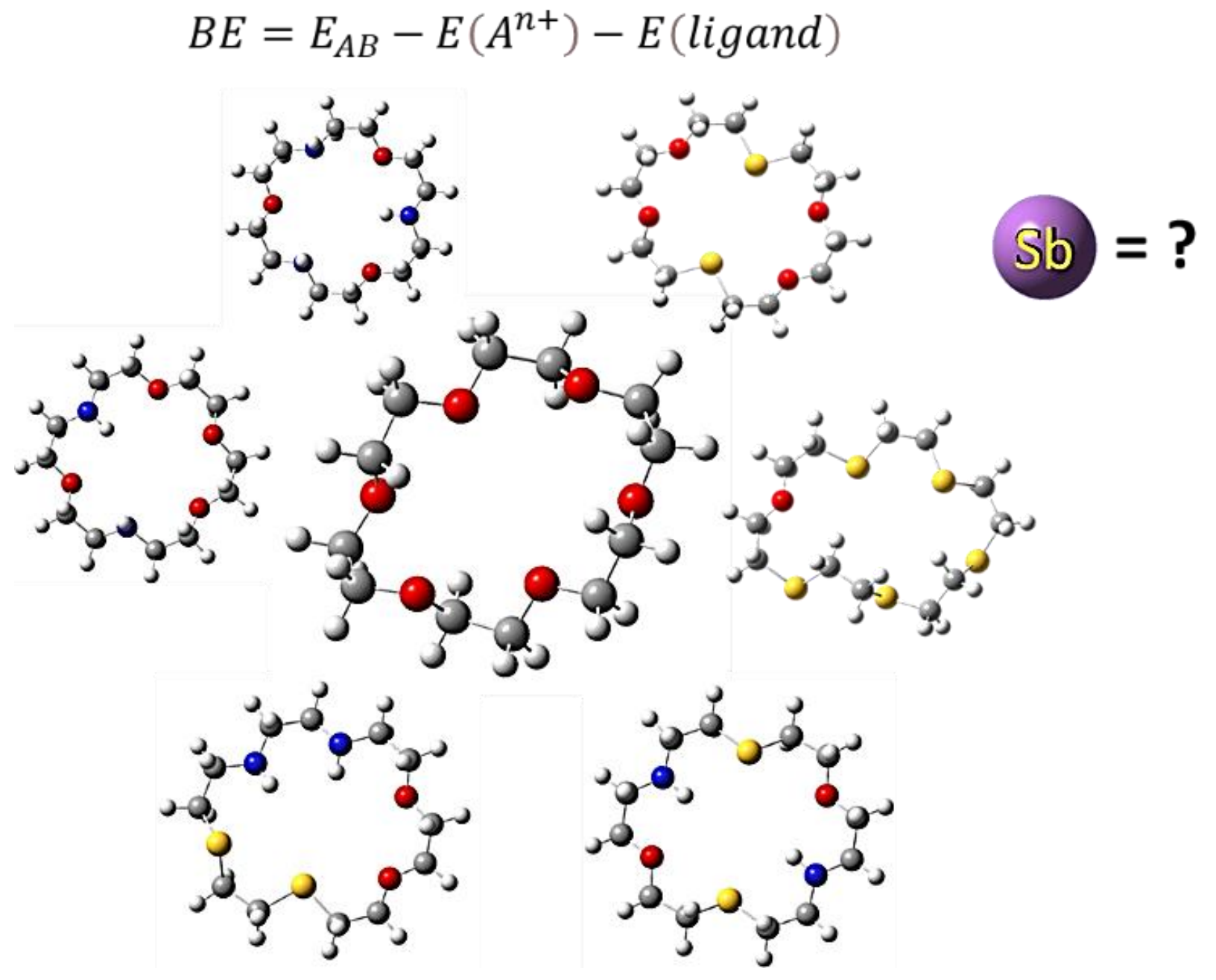

Investigations into host-guest interactions with metal ions using DFT for applications using mass spectrometry 


\begin{abstract}
The versatility and applicability of supramolecular (host-guest) chemistry span multiple disciplines such as environmental, medical, and, more recently, forensic applications. Among these molecules, crown ethers can associate with metal ions which can be characterized and confirmed using high-resolution mass spectrometry techniques. However, in specific situations, host-guest interactions in complex matrices are complicated by competitive binding and other factors that influence complex formation. For example, metalloids like antimony do not readily associate with macrocycles whilst in the presence of other metals such as barium and lead. Two main questions arise from this observation: 1) Which host molecule binds the most efficiently to metal species? 2) What are the main factors most affected by binding? Here, density functional theory (DFT) simulations were utilized with LC-MS/MS to predict behaviors in common solvents and monitor binding selectivities. Structures were generated and modeled after 18-crown-6-ether, including hexacyclen, several aza-crowns, and thia-crown ethers. Additionally, different heteroatoms mixtures were included to monitor the effects on the binding energies. Strategies like natural bond orbital (NBO) analysis, HOMO-LUMO energy gaps, and electrostatic potentials contributed to describing interactions in the metal-ligand complexes. Metal species with varying metal charges such as $+1,+2$, and +3 were tested in gas and solvent phases at varying protic and aprotic strengths (water, methanol, acetonitrile). Out of the factors investigated, the chemical hardness and reactivity of the metal species affected the binding of $18 \mathrm{C} 6$.
\end{abstract}

Keywords: Host-guest interactions, NBO analysis, Exploratory analysis, binding energies 


\subsection{Introduction}

Various disciplines, including environmental, medical, and even forensic sciences (specifically gunshot residue (GSR)), often require the monitoring and characterization of inorganic and organic constituents from an unknown sample [1-4]. Typically, these analyses require multiple instruments and methodologies, which while effective, can be cumbersome and labor-intensive. The typical approach for organic analysis combines chromatographic techniques with mass spectrometry analyzers (most commonly liquid chromatography-tandem mass spectrometry (LC-MS/MS)) for comprehensive and quick analysis of complex mixtures. In cases where inorganic species are targeted, strategies such as chelation or host-guest complexes can be utilized to expand the detection of LC-MS systems $[5,6]$. The challenges associated with those two approaches, however, lie within the matrix in which multiple species compete for host molecules.

When developing systems for multi-analyte analysis, specific assays require careful selection to encompass complexing agents as well as an understanding of competitive interactions and stoichiometry. Additionally, choosing one "optimal" complexing agent is inherently challenging as there are not only a myriad of complexing agents but multiple variants of those agents available $[7,8]$. One technique that can alleviate the screening process is via molecular simulation/modeling which can ultimately predict potential host-guest complexation. Such strategies can assess competitive interactions, shed light on how complexes are likely to be solvated in a liquid chromatographic environment, and can create novel/unique structures that can address specific research inquiries. Amongst one of the most commonly macrocycles or host agents is the crown ether species, which is the central focus of this study.

The discovery of crown ethers through Pedersen's, Cram's, and Lehn's works sparked interest within the scientific community and promoted the expansion of supramolecular chemistry 
into other fields [8]. Crown ethers belong to a subclass of macromolecules known as cryptands or cryptates, which are cyclic structures containing heteroatoms (oxygen, nitrogen, sulfur) and other functional groups modifications to capture analytes or guests. The simplicity of a crown ether structure allows for extreme flexibility and versatility, facilitating its use across various disciplines [9-13]. More complex macrocycles such as cucurbit[n]urils and cyclodextrins build upon the knowledge gained from crown ethers and have been utilized in research and medical needs ranging from drug delivery to chemo-sensing and point-of-care devices to encapsulate larger guests $[8,14,15]$.

The mechanisms associated with macrocycles vary depending on the guests present in the surrounding environment and how they interact via intermolecular forces such as ion-dipole, noncovalent, electrostatic, and van der Waals forces $[8,16]$. These interactions are influenced by lone electron pairs from the contributing heteroatoms (oxygens) and create non-covalent, electrostatic bonds between cations. Depending on the macromolecule's size and internal composition, binding selectivity and affinities affect guests differently [13,17-20]. Additionally, these simple ionophores can form "sandwich" structures, which are stoichiometric ratios of a 2:1 host-to-guest combination. However, the 1:1 H-G structures are more common when associated with metal ions due to the flexibility of simplistic macrocycle composition [21].

Crown ether macrocycles, whose inner cavities are large enough to house guests, can selfassemble to create combinations with metals in the form of metal-ligand (M-L) complexes. Experimentalists have observed M-L complexes by preserving precursor structures analysis using electrospray ionization mass spectrometry (ESI-MS) $[11,18,21-23]$. This technique is appealing in several ways, including low sample consumption, the survival of many types of weakly bound complexes, use of common solvents, and the versatility of coupling to various instrumentation 
methods. These phenomena observed by ESI-MS offers crucial insights into binding affinities and structural confirmations. However, recent advances in molecular modeling may give a more indepth investigation into the underlying forces of binding heavy metals. Hence, these approaches were utilized for the investigation of a complex mixture of inorganic species found in gunshot residue (GSR). As a result of heavy use of firearms in the U.S., these residues can become important for forensic, environmental, and public safety scientists.

Recent work performed focused on leveraging host-guest interactions for forensic applications to monitor isotopic distributions of three heavy metals (lead $(\mathrm{Pb})$, barium $(\mathrm{Ba})$, and antimony $(\mathrm{Sb}))$ for gunshot residue classification [2,11]. Additionally, these metals exist in various forms including sulfides, nitrates, hydroxides, as well as others further complicating potential characterization [1]. The combination of these elements exists as a physical conglomerate and thus, must be dissolved via concentrated acids like $\mathrm{HCl}$ and $\mathrm{HNO}_{3}$ for interactions to exist. Both $\mathrm{Pb}$ and Ba readily self-assembled with 18-crown-6-ether (18C6) with Ba being more readily bound versus $\mathrm{Pb}$ ions. Both $\mathrm{Pb}$ and $\mathrm{Ba}$ self-assembled with 18-crown-6-ether (18C6) with Ba being more readily bound versus $\mathrm{Pb}$ ions. Previous LC-ESI-MS/MS studies demonstrated Sb did not readily interact with the 18C6 macrocycle as well as other hosts like 15C5 and hexacyclen. However, Sb forms with a multidentate chelating agent, such as tartaric acid [2]. This observation was highlighted by Wijeratne's work which combined information obtained from negative ESI (-) mode in conjunction with DFT [24-27].

Computational strategies like quantum mechanical and molecular dynamic methods offer structural insights for crown ethers with metal ions in both gas and solution phases as well as membrane systems [28-31]. Most groups complexed with crown ethers span from alkali metals (Group I) and alkaline earth metals (Group II) to other transition metals [2,32-34]. However, 
molecular dynamic studies estimate transition metals' properties based on calculated force fields which originate from Newton's second law [35]. Therefore, a metal not described by a force field may lead to inadequate descriptions of the M-L complexes and furthermore the underlying mechanisms for formation. Quantum mechanical models like density functional theory (DFT) circumvent these shortcomings by applying various functionals to describe an electron's density.

Evidence of DFT analysis' effectiveness has been demonstrated by various groups and fields in describing the electronic structure of atoms, molecules, and other uses such as drug encapsulation and spectral monitoring [30,36-40]. Amongst ab initio literature for crown ethers, the predominant basis set for the base macrocyclic structure is the Becke three-parameter exchange functional engineered from Lee, Yang, and Parr (B3LYP) [33,41,42]. The accuracy for these functionals increases by hybridizing with pseudo-potentials on the heavy elements, and the valence basis set augmented with polarization functions. For example, the 6-311G++(2d, p) basis set characterizes the crown ether $\left(\mathrm{C}_{12} \mathrm{H}_{24} \mathrm{O}_{6}\right)$ and potential heteroatoms such as nitrogen $(\mathrm{N})$ and sulfur (S), whereas the basis set developed by Los Alamos National Laboratory (LANL2DZ) describes the metallic species. Using this combined approach, one can investigate a multitude of properties including thermochemical characteristics, natural bond orbital (NBO) analysis, HOMO-LUMO energy gaps, and molecular electrostatic potentials (MESP) which can describe factors influencing binding affinity. In addition, other properties such as electronegativities $(\chi)$, hardness $(\eta)$, and energy gaps can provide more insight into transition metal interactions.

For computational approaches, gas-phase calculations are the most performed methods to visualize and study these interactions. However, the solvation of these species should also be studied to potentially investigate structural effects in more realistic conditions. There are two types of solvation strategies that can describe analytes in solution - explicit and implicit strategies. 
Explicit solvation models employ hundreds of solvent molecules acting within a system whereas implicit models treat solvents as a singular polarizable medium $[43,44]$. Here, the implicit strategy called Solvation Model based on Density (SMD) was employed which treats the solvent as a dielectric constant of the selected solvent $[43,45,46]$. SMD has proven to be an effective solvation model for use in both charged and uncharged systems and is able to predict accurate solvation energies for various functional groups [46].

The present work aims to expand upon the current knowledge of the crown ethers' versatility and selectivity in complex and competitive environments using barium (Ba), antimony $(\mathrm{Sb})$, and lead $(\mathrm{Pb})$ as the example analytes. Additionally, the formation of "sandwich" structures was investigated by observing the displacement of the metal ion within the inner cavity of the 18C6 and heteroatoms. Here, heteroatom substitutions were monitored using density functional theory (DFT) to observe the potential reactions and limitations amongst affinities for different ions. To evaluate these interactions more objectively, exploratory analysis methods and correlations will be drawn using variables such as metal species size, charge, and the host molecule's composition (number of $\mathrm{O}, \mathrm{N}, \mathrm{S}$ ) atoms were used to evaluate possible correlations.

\subsection{Experimental Section}

\subsubsection{High-Performance Computing Cluster and instrumentation methodology}

All simulations were performed using the High-Performance Cluster (HPC) Thorny Flat at West Virginia University (WVU). Gaussian16 ${ }^{\circledR}$ and NBO $3.1 \circledR$ were performed on the cluster and visualized using GaussView6®. For the experimental portion, both an Agilent 1290 Infinity II liquid chromatography coupled to a 6470-triple quadrupole (QQQ) mass analyzer and a Thermo Fisher Scientific Q-Exactive ${ }^{\circledR}$ orbitrap analyzed compounds using flow-injection analysis (FIA). The Q-Exactive Orbitrap was utilized to observe the isotopic distribution of the inorganic elements 
as present when exposed to the crown complex. No chromatographic analysis was conducted since the Orbitrap was utilized as a confirmation tool using direct infusion approach. The observation of the natural abundances for studied elements serve as confirmation of their presence in the complex agent.

\subsubsection{First-Principles Simulation Methodology}

For the core structures of the ligands, the B3LYP functional with a 6-311++G (2d, p) was utilized in both gas and solution phases for carbon $(\mathrm{C})$, hydrogen $(H)$, oxygen $(\mathrm{O})$, nitrogen $(\mathrm{N})$, and sulfur (S). The LANL2DZ effective core potential (ECP) was employed for all transition metals to monitor the inorganic complexes in both gas and solution phases. Structures were constructed using the 18C6 base skeletal structure as a reference, outlined in Figure 6.1, in the Avogadro (open-source version 1.2) software for initial connectivity and energy minimization. The .mol2 files were then converted to the gaussian format and were analyzed using Gaussian $16 \AA$ (Gaussian, Inc., Wallingford, CT, USA). Both geometry optimizations and frequency calculations were performed both in gas and solution phases (water, acetonitrile, and methanol) and were visualized using GaussView 6.0®. 


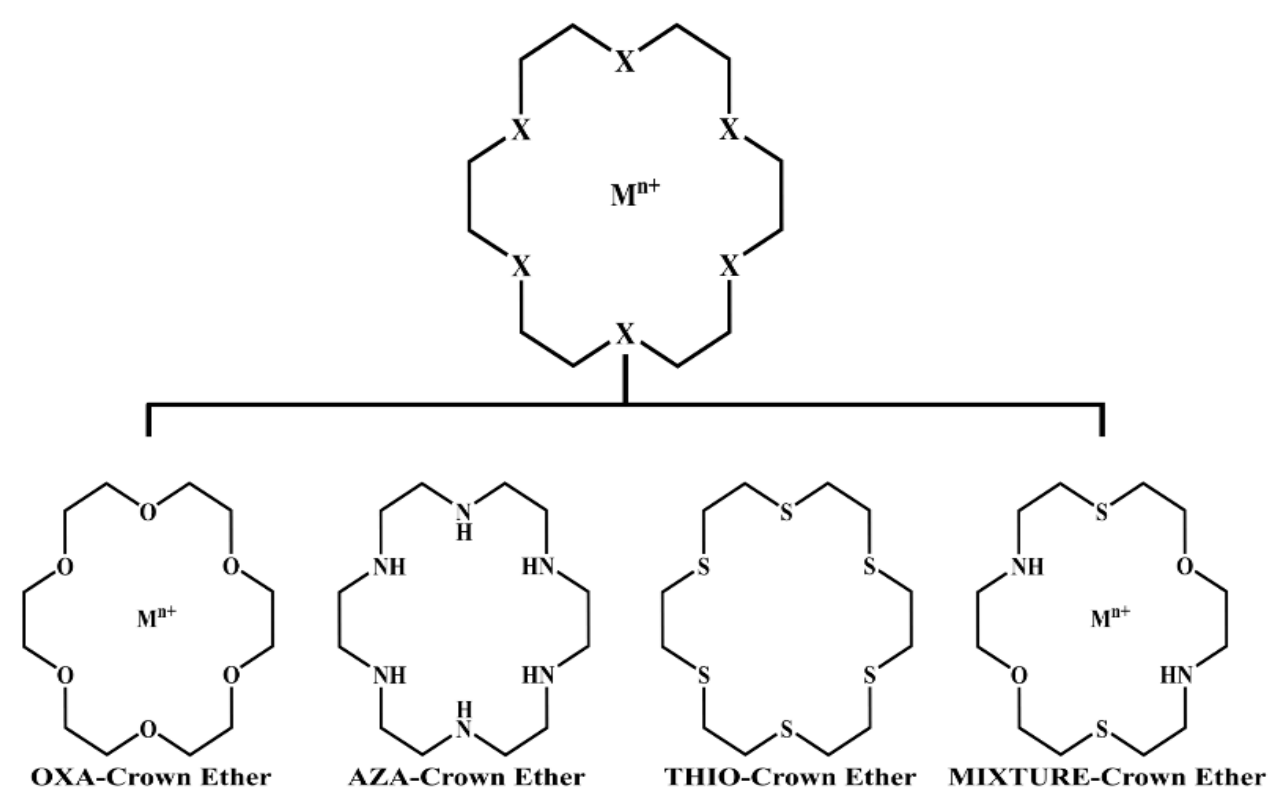

Figure 6.1: Skeletal structure design of varying crown ethers simulated. Starting from the base structure of 18-crown-6-ether (18C6), nitrogen and sulfur heteroatoms were added incrementally.

The binding energy $\left(B E_{E}\right)$ or binding affinity accounts for the energy contributed by the host agent and the guest analyte. This phenomenon is calculated by Equation 6.1 :

$$
B E_{E}=E_{A B}-E\left(A^{n+}\right)-E(\text { ligand })
$$

Equation 6.1

where $\mathrm{E}_{\mathrm{AB}}$ is energy of $[\mathrm{M}(18 \mathrm{C} 6)]^{\mathrm{n}+}$ complex, $E\left(A^{n+}\right)$ is the energy of metal cation under consideration, and $E$ (ligand) is the energy of 18-crown-6 macrocycle. These interaction energies were calculated without zero-point energy (ZPE) correction, ZPE. An important note when calculating and detailing the intricacies with M-L interactions is mixing basis sets. Typically, atoms are susceptible to basis set superposition error (BSSE), which occurs when two atoms approach and overlap each other [47]. This effect increases as the atoms orient themselves closer, creating an effectively varying basis set against interatomic distance. Equation 6.1 accounts for the interaction error by considering the energy contributions from the LANL2DZ and 6-311++G (2d, p) basis sets. 
Solutions were kept constant to monitor any potential distortions while simultaneously mimicking potential sample environments. The aqueous and organic solvent environments were modeled using an implicit polarizable continuum model called Solvation Model based on Density (SMD) to help monitor electrostatics contributing from solvents. Three different solvent environments water, methanol, and acetonitrile with varying polarizabilities $(\varepsilon=78.39,32.61$, and 35.69 , respectively) were used to monitor effects in structures and binding affinities. Additionally, the host molecules and M-L complexes solvation are calculated (Equation 6.2) which involves three aspects when calculating: electrostatics, dispersion/repulsion forces, and the cavitation energy.

$$
G_{\text {solvation }}=G_{\text {electrostatic }}+G_{\text {dispersion-repulsion }}+G_{\text {cavitation }} \quad \text { Equation } 6.2
$$

From there, the free energies of the entire interaction, both gas and solution phases, can be calculated by

$$
\Delta G_{\text {solvation }}=G_{\text {solvent }}-G_{\text {gas-phase }}+\text { BSSE } \quad \text { Equation } 6.3
$$

Where the thermochemical responses from the solution and gas phases while also considering the basis set superposition error. Other factors under observation include the electronegativity, chemical hardness, energy gaps between HOMO and LUMO, ionization potential, and potential transfer of charges using natural bond orbital (NBO) analysis. Although these factors may contribute less to the overall binding affinity for groups such as the alkali and alkali earth metals, these factors may be more influential for the pnictogens or metalloid. Therefore, combination of $o x a-, a z a-$, thia-, and different combinations of heteroatoms within the macrocycles' structure was also investigated to elucidate which factors invoke different responses. 


\subsubsection{Natural bond orbital (NBO) calculations}

To further explain the factors contributing to metal binding selectivity, NBO analysis was employed to investigate frontier molecular orbitals (LUMO-HOMO) energies for 18C6 with the selected transition metal ions to assess the stability of the metal binding pocket. The NBO 3.1 program along with NBO the Gaussian(C) 16 calculated the energy eigenvalues based on the molecular orbital theory. Semi-quantitative data from simulations such as chemical hardness/softness can be studied using Klopman's theorem [48] and the finite difference approximation. Parr and Pearson have defined the chemical hardness $(\eta)$ [49] as

$$
\eta=\frac{1}{2}(I E-E A) \quad \text { Equation } 6.4
$$

where IE and EA are the vertical ionization potential and vertical electron affinity, respectively. The energy of the highest occupied orbital $\left(E_{\mathrm{HOMO}}\right)$ is referred to as the ionization potential $(I E)$ and the lowest unoccupied orbital $\left(E_{\mathrm{LUMO}}\right)$ to electron affinity $(E A)$. Other factors like chemical softness $(S)$ stems from the hard/soft acids/bases (HSABs) principle made by Pearson and to help explain stabilities in Lewis acid/base reactions [49,50]. Later, Klopman tried to quantify the HSAB principle by using terms from ionic interaction (contribution from charge-controlled reaction) and covalent interaction (contribution from frontier molecular orbital) [50]. This indicator can assist in understanding electron localization over the covalent bonding of the metal ion complexes and can be defined as:

$$
S=\frac{1}{2} \eta
$$

Equation 6.5

\subsubsection{Second order perturbation theory}

The values of the stabilization energies in NBO analysis, namely the second-order interaction energies, $E_{i j}{ }^{2}$, are closely related to the strength of the coordination interaction. A complex will, generally, be more stable if it has a large corresponding stabilization energy $E^{2}$. The 
natural bond orbital (NBO) analysis provides a description of a structure by a set of localized bond, anti-bond and Rydberg extra valence orbitals [51-53]. Delocalization of electron density between occupied Lewis-type (bond or lone pair) NBO orbitals and formally unoccupied (anti-bond or Rydberg) non-Lewis NBO orbitals corresponds to a stabilizing donor-acceptor interaction, which is taken into consideration by examining all possible interactions between filled (donor) and empty (acceptor) orbitals, and then evaluating their energies by second-order perturbation theory $[33,53]$. For each donor $(i)$ and acceptor $(j)$, the delocalization is estimated by the following equation:

$$
E^{2}=\Delta E_{i j}=\frac{q_{i} F(i, j)^{2}}{\varepsilon_{j}-\varepsilon_{i}} \quad \text { Equation } 6.6
$$

Where $q_{i}$ refers to the $i$ th donor orbital occupancy, $\varepsilon_{i}$ and $\varepsilon_{j}$ are the diagonal elements (orbital energies), and $F(i, j)$ is the off-diagonal element. The higher the value of $E^{(2)}$, the more the intermolecular orbital interactions and consequently greater charge transfer between the electron donors and the electron acceptors (between the binding pocket of the macrocycles and metal ions).

This type of analysis helps illustrate the interaction energies by calculating the magnitude of charge transfers between a host and guest. This can help detail the contributing factors for electrostatic interactions by investigating lone pairs (LP), bonding orbitals (BD), and core electrons (CR) as well as antibonding properties (LP*) [54]. Through this, trends between structural changes and binding energies can be observed which then can lead to developing selective ligands.

\subsection{Results/Discussion}

\subsubsection{Confirmation of Structures via High-resolution Mass Spectrometry}

Within our laboratory, initial interest to investigate these complex mixtures stemmed from forensics applications for efficient dual detection and chemical characterization of GSR. After a discharge event, two sets of constituents disperse into the surrounding environment [1]. These 
analytes comprise of both organic molecules and inorganic species in which latter involves three essential elements ( $\mathrm{Pb}, \mathrm{Ba}$, and $\mathrm{Sb})[1]$. These inorganic analytes exist as a conglomerate of physical particulates house more elements, which presents the possibility for competition for these macrocycles. Recently, a common instrumentation technique (LC-MS/MS) was validated as it demonstrated its effectiveness to identify and characterize the IGSR analytes complexed with $18 \mathrm{C} 6[2]$.

For additional confirmation of these M-L complexes, preliminary experiments involved the use of an orbitrap high-resolution mass analyzer [2,11]. These observations verified the ability of 18C6 to self-assemble into a 1:1 host:guest stoichiometric ratio with two metal species, Ba and $\mathrm{Pb}$. Figure 6.2 shows mass spectra of two equimolar solutions of $\mathrm{BaNO}_{3}: 18 \mathrm{C} 6$ and $\mathrm{PbNO}_{3}: 18 \mathrm{C} 6$ from ICP-MS standards containing trace amounts of nitric acid. To compare the efficiency of the M-L complexes, all major isotopes were compared for both metal analytes. The total ion signal across the major isotopes of the $1: 1 \mathrm{BaNO}_{3}: 18 \mathrm{C} 6$ complex was approximately $1.3 \times 10^{9}$ whereas the $1: 1 \mathrm{PbNO}_{3}: 18 \mathrm{C} 6$ complex was approximately $1.0 \times 10^{9}$. This difference in absolute abundances suggests that the complex stability and/or ionization efficiency are $\sim 30 \%$ greater for the crown ether complex containing $\mathrm{Ba}$ relative to $\mathrm{Pb}$.

With the confirmation via collision induced dissociation (CID), the LC-MS/MS was validated and tested amongst various scenarios Thallium (Tl) was introduced to a mixture as an internal standard since it is not observed in either traditional or non-toxic ammunition. Two metal ions $\left(\mathrm{Ba}^{2+}\right.$ and $\left.\mathrm{Tl}^{+}\right)$were introduced in an acetonitrile solution at equal concentrations and were then exposed to $18 \mathrm{C} 6$ at a stoichiometric ratio of 2:1:1. The overall response of $\mathrm{Ba}$ was lowered with the addition of Tl, represented in Figure S6.1, suggesting that competition between guests is prevalent and all respective signals should be considered when evaluating selectivities of 
macrocycles. Upon further investigation, the $18 \mathrm{C} 6$ preferred $\mathrm{Tl}^{+}>\mathrm{Ba}^{2+}>\mathrm{Pb}^{2+}$ which has been observed by Zhao et al.[13] comparing $\mathrm{Tl}^{+}$and $\mathrm{Pb}^{2+}$.
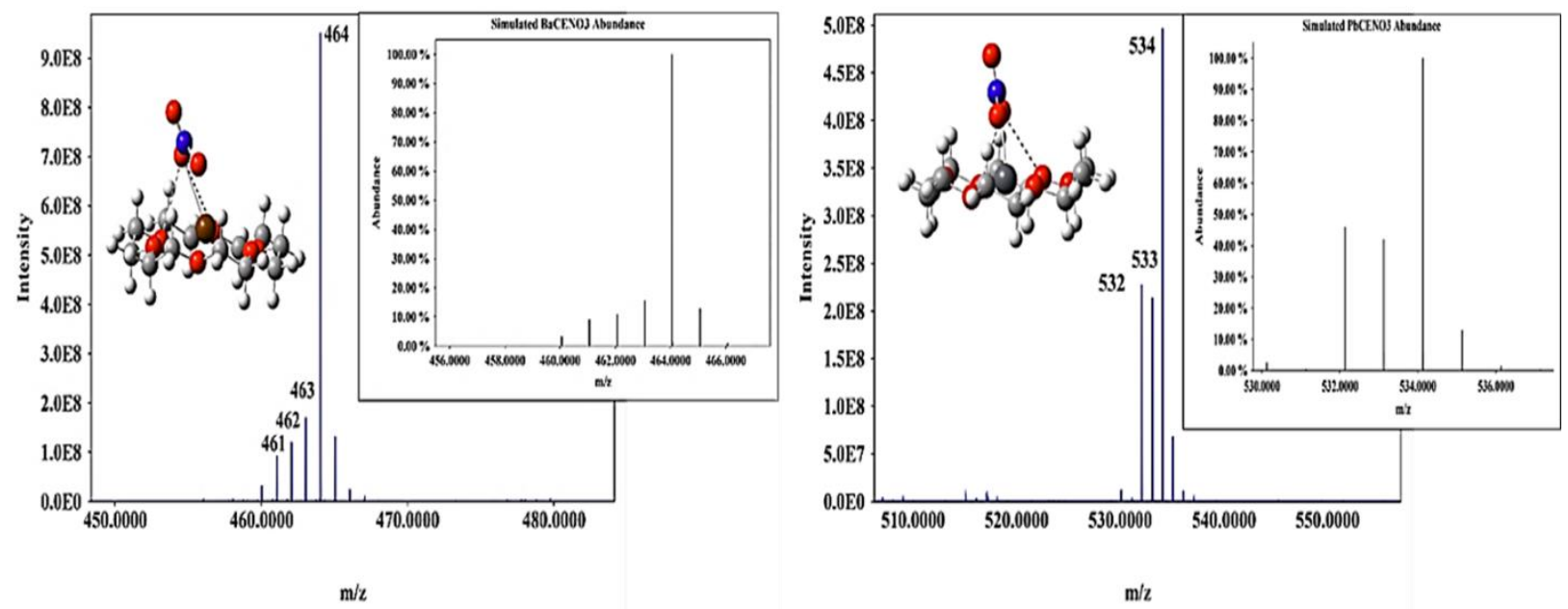

Figure 6.2: Collected and simulated isotopic abundances from orbitrap of crown ether complexes for $\left[\mathrm{BaNO}_{3}\right]$ (left) and $\left[\mathrm{PbNO}_{3}\right]$ (right).

Throughout those preliminary investigations, however, it was concluded that $\mathrm{Sb}$ did not complex with $18 \mathrm{C} 6$ or other macrocycles such as $15 \mathrm{C} 5$ or even hexacyclen $[2,11]$. However, scientists successfully identified and characterized $\mathrm{Sb}$ through the process of chelation which forms physical bonds with electron-donor atoms such as sulfur, nitrogen, and/or oxygen [3]. This strategy has been used for the removal of heavy metals in a variety of applications including wastewater treatments and pharmaceutical uses [3,6,55]. One commonly used chelator is ethylenediaminetetraacetic acid (EDTA) which has effective removed different metals from various scenarios [56]. In terms of identifying antimony, tartaric acid follows this mechanism by forming multidentate bonds, which can be observed in negative ESI (-) mode [25]. This phenomenon was illustrated by Schug's group where tartaric acid was investigated both experimentally and computationally [24-27].

Recently, this chelating agent was used for forensic uses but with limited success achieved due to potential low concentrations of Sb present in GSR particulates [2,11]. Interestingly, when 
$\mathrm{Ba}, \mathrm{Pb}$, and $\mathrm{Sb}$ were present in one solution, tartaric acid was more selective for $\mathrm{Sb}$ than the other metals. However, Sb-TT in ESI (-) mode would be difficult to identify in complex matrices because the isotopic distributions are altered from radical recombination and homolytic cleavages from the ligand [24]. Thus, efforts shifted to find an optimum macrocycle that not only can uptake these analytes but to also preserve their natural isotopic abundances.

\subsubsection{Evidence of Sandwich complexes}

Initially, the selectivity of crown ethers to metal cations were often described as a "best-fit" or "lock-and-key" principle based on the metal cation size and a crown ether's inner cavity [57]. However, the high flexibility of these ionophores makes this view limited when presented with a larger inner cavity such as $18 \mathrm{C} 6$. Other factors that have shown influential to affect binding affinity are charge on the most prominent ion charge $(z)$, energy for ionization $\left(E_{i}\right)$, and their electronegativities $(\chi)$. Depending on these characteristics, there is a possibility to form a 2:1 complex due to the cation's positioning in relation to the size of the ionophore inner cavity $[21,32,58]$. Interestingly, these structures preserve the natural isotopic abundances of the captured metal species, shown in Figure 6.3, which again illustrates the ease of identifying structures. However, these complexes are dependent on stoichiometric ratios within a solution and thus are less observed [21,58]. Therefore, the remaining structures focused on the 1:1 M-L stoichiometric ratios and their response to various solvents and heteroatom substitutions. 


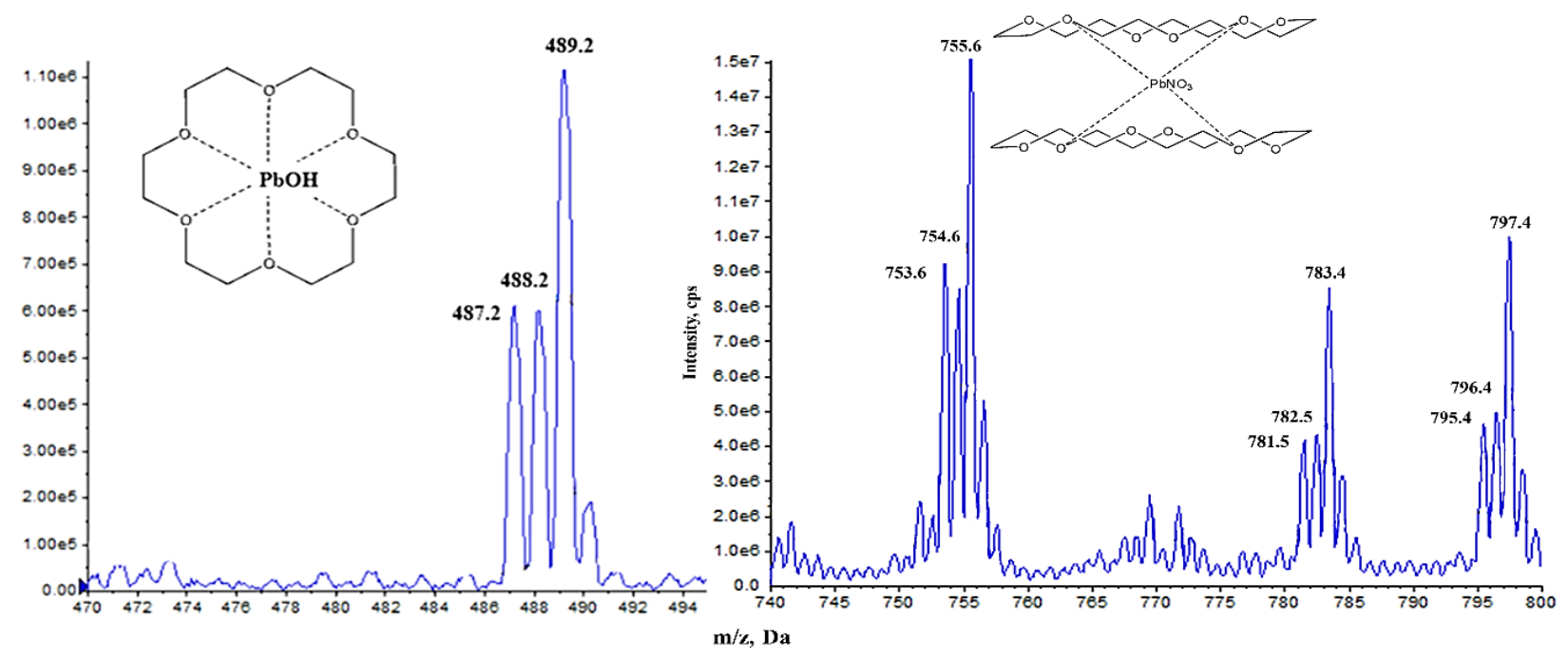

Figure 6.3: Experimental example of the formation and CID results from a 1:2 M-L complex to a 1:1 M-L complex in ESI (+) mode. In both instances, the natural abundances of $\mathrm{Pb}$ are preserved even after the loss of one host molecule

The geometry optimizations of the lone ligands were calculated at the $6-311++\mathrm{G}(2 \mathrm{~d}, \mathrm{p})$ level whereas the M-L structures utilized a hybrid electron core potential (ECP) using the LANL2DZ basis set for the metal species. Several cations were selected due their varying charges and properties that may contribute to selectivity of the macrocycles, recorded in Table 6.1. Note that cations such $\mathrm{Na}, \mathrm{K}$, and $\mathrm{Ca}$ are often found in a range of samples and thus should be considered as competitive species in most assays.

Table 6.1: Detailed properties of cations under investigation to interact with the generated ligands.

\begin{tabular}{c|ccccccc}
\hline Cation & $z$ & $E_{i}$ & $\chi$ & $\mathrm{d}$ & $z / \mathrm{d}$ & $E_{i} / \mathrm{d}$ & $\chi / \mathrm{d}$ \\
\hline $\mathrm{Na}^{+}$ & 1.00 & 0.496 & 0.93 & 1.94 & 0.515 & 0.256 & 0.479 \\
\hline $\mathrm{K}^{+}$ & 1.00 & 0.419 & 0.82 & 2.74 & 0.365 & 0.153 & 0.299 \\
\hline $\mathrm{Tl}^{+}$ & 1.00 & 0.589 & 1.62 & 3.00 & 0.333 & 0.196 & 0.540 \\
\hline $\mathrm{Ba}^{2+}$ & 2.00 & 0.965 & 0.89 & 2.70 & 0.741 & 0.357 & 0.330 \\
\hline $\mathrm{Pb}^{2+}$ & 2.00 & 1.451 & 2.33 & 2.38 & 0.840 & 0.610 & 0.979 \\
\hline $\mathrm{Sr}^{2+}$ & 2.00 & 1.064 & 0.95 & 2.36 & 0.847 & 0.451 & 0.403 \\
\hline $\mathrm{Mg}^{2+}$ & 2.00 & 1.45 & 1.31 & 1.14 & 1.754 & 1.272 & 1.149 \\
\hline $\mathrm{Ca}^{2+}$ & 2.00 & 1.145 & 1.00 & 2.00 & 1.000 & 0.573 & 0.500 \\
\hline $\mathrm{Ga}^{3+}$ & 3.00 & 2.963 & 1.81 & 0.94 & 3.191 & 3.152 & 1.926 \\
\hline $\mathrm{In}^{3+}$ & 3.00 & 2.704 & 1.78 & 1.24 & 2.419 & 2.181 & 1.435 \\
\hline $\mathrm{Sb}^{3+}$ & 3.00 & 2.44 & 2.05 & 1.52 & 1.974 & 1.605 & 1.349 \\
\hline
\end{tabular}


Where $z$ represents the most prominent ion charge, $E_{i}$ is the ionization energy, $\chi$ is the electronegativities, and $d$ is the diameter given in angstroms $(\AA)$. Additionally, each of those properties were calculated for the ratio to the cation diameter to provide insight into potential factors that can affect the binding affinity of the generated macrocycles. Those factors were then related to the cation's diameter to monitor which property is affected by the internal diameter of the ligand.

The ions outlined in Table 6.1 were chosen for multiple reasons expanding from forensic analytes-of-interest to proteomic concerns and environmental pollutants. For instance, analytes studied using ESI typically form $\mathrm{Na}$ and $\mathrm{K}$ adducts which can originate from a variety of sources including leaching from glassware or additives found in mobile phases [59]. These ions, when not checked, can cause hinderances to not only analysis of larger biomolecules (i.e., supercharging proteins) but can damage LC and source conditions [60,61]. Elements such as calcium (Ca) and magnesium $(\mathrm{Mg})$ are of interest as they are important to health and pharmaceutical considerations. Indium (In) and gallium (Ga) were utilized to help monitor the size and charge contribution for Sb. Finally, the remaining elements (strontium $(\mathrm{Sr})$, barium $(\mathrm{Ba})$, lead $(\mathrm{Pb})$, and thallium $(\mathrm{Tl}))$ are of interest because these metals can be found in wastewater. Hence, the need for treating and extracting these species without further contaminating the sources is critical.

\subsubsection{Influence of solvent effects on structure}

When investigating host-guest interactions, an important aspect to consider is how the solvent influences a structure's conformation as well as binding energy. There are two strategies in which can describe an analyte within a solvent system - explicit and implicit. The main difference between the two are as follows - Implicit solvent models treat solvents as a continuous medium surrounding the solute, whereas explicit models take into account the movements and 
effects of the actual solvent molecules within a given region around the solute molecules. Because of how these complexes are observed experimentally, differences in polarity and $\mathrm{pH}$ conditions may disrupt the responses of these host-guest interactions. However, experimentalists have utilized ESI as it has proven to allow the survival of many types of weakly bound complexes while maintaining the natural properties of the analytes [21,62]. Additionally, ESI tends to produce sodium (Na) and potassium (K) adducts resulting from the typical solvents used in LC mobile phases as well as water adducts [59]. However, we did not observe any water adducts with the ML complex in our experimental results thus, the implicit solvation strategy was chosen as polarizability can also affect the conformation of these M-L complexes. Three solvents (water, methanol, and acetonitrile) with varying polarizabilities ( $\varepsilon=78.39,32.61$, and 35.69 , respectively $)$ were monitored due to their wide availability for experimental purposes (i.e., dissolving, highresolution mass analyzers, source conditions). 
Gas

$18 \mathrm{C} 6$

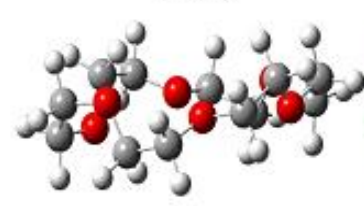

18C6

$\mathbf{K}^{+}$

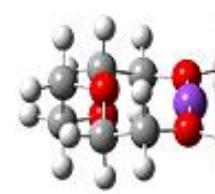

d

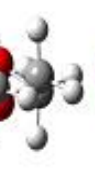

Acetonitrile

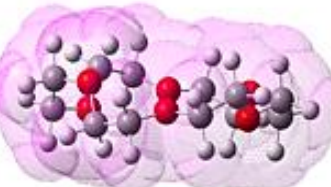

Methanol
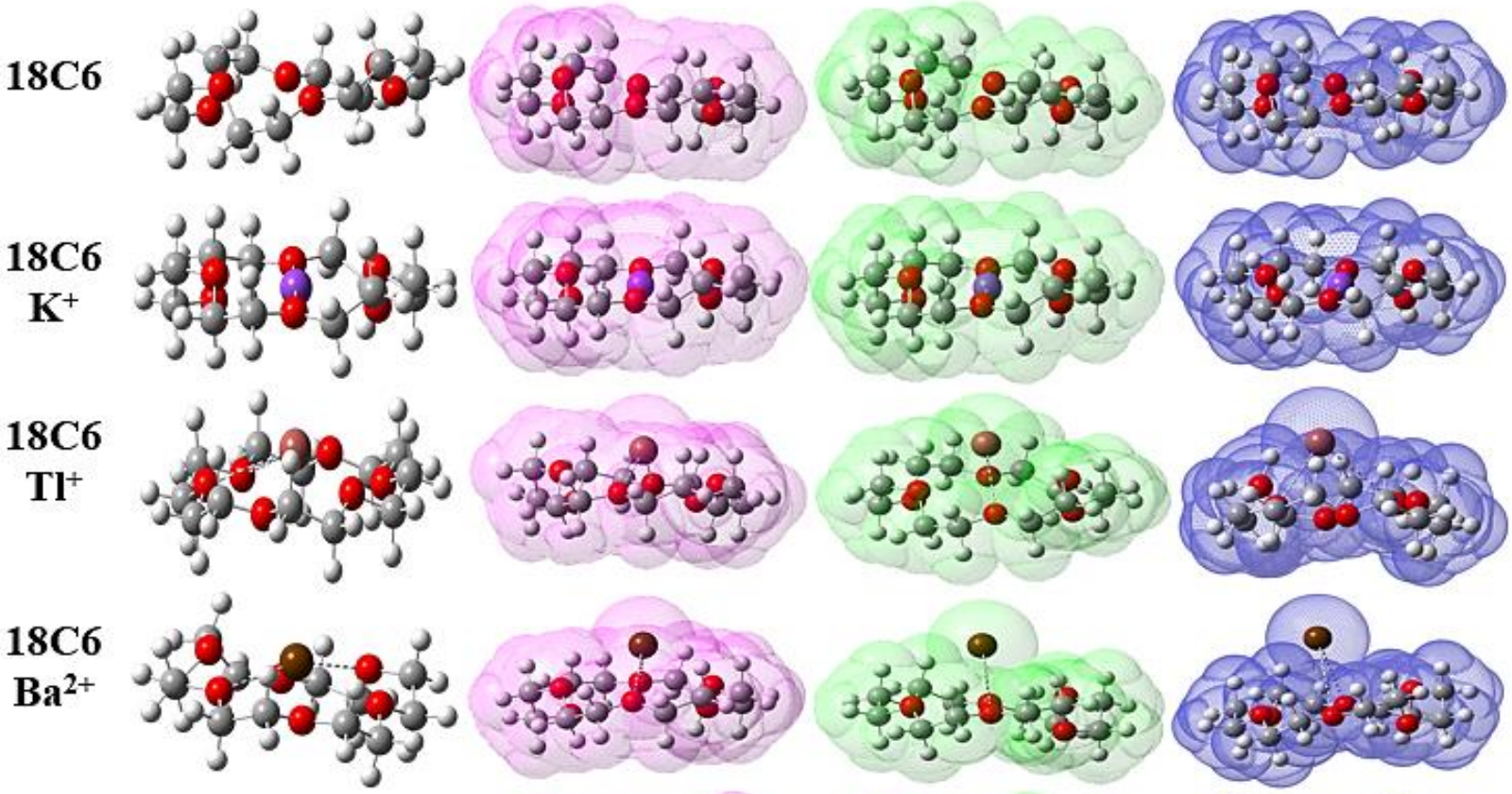

$18 C 6$
$\mathrm{~Pb}^{2+}$
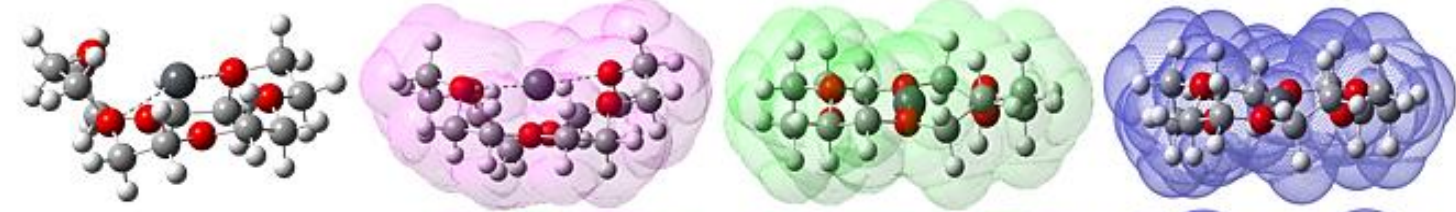

$18 \mathrm{C6}$
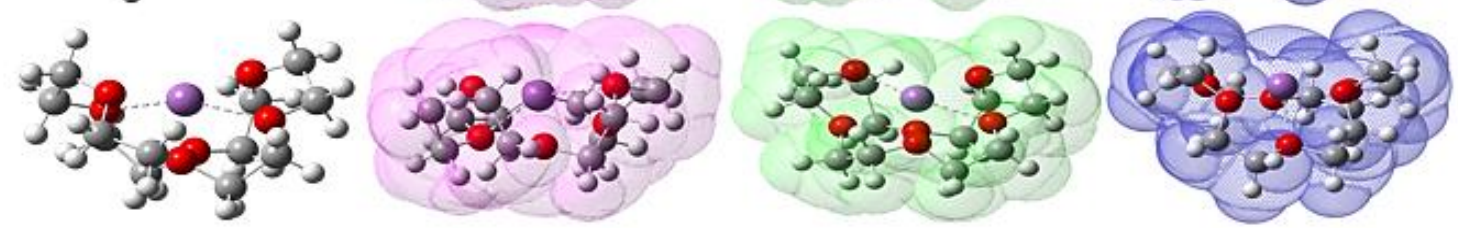

Figure 6.4: Energy minimized structures of various M-L complexes with different charges of guests in both solvent (SMD) and gas phases. Both phases (gas and solution) were modeled to investigate any potential structural and binding effects. The solvents acetonitrile (magenta), methanol (green), and water (blue) were chosen for their commonality in dissolving crown ethers in instrumentation parameters.

Initially, 18C6 was investigated due to its relatively larger inner diameter (2.7-3.2 Å) [63], its high flexibility, and work previously performed in our laboratory. Crown ethers and other larger macrocycles like the cyclodextrins and cucurbit[n]urils possess the capabilities to self-assembled with a variety of guest species $[16,64,65]$. To highlight these properties using quantum mechanical models, however, solvation strategies must be utilized to monitor any conformational changes within each scenario. Depending on the solvation strategy (implicit or explicit), researchers can 
investigate different responses of larger biomolecules and even spectroscopy sensing structures $[38,44]$. Here, however, the solvent was treated as smooth continuous model to determine the differences between the surface of the simple macrocycles within polarizable mediums [66].

The structures of the 18C6 and the M-L with various metal charges freely reorient and form around the metal species. In both gas and solution phases, Ba showed the largest displacement from the internal cavity of 18C6 (Figure 6.4, row four), whereas $\mathrm{Pb}$ showed the largest difference between the acetonitrile and methanol. This is particularly interesting as these two analytes were both capable of forming "sandwich" complexes (Figure 6.4). Like the experimental results in Figure S6.1, 18C6 favors Tl over Ba and Pb yielding energies (Hartree) of the complex of -974.48, -947.86 , and -925.97 respectively giving a trend of $\mathrm{Tl}>\mathrm{Ba}>\mathrm{Pb}$. Furthermore, $18 \mathrm{C} 6$ demonstrated that it could encapsulate $\mathrm{Sb}$ in all scenarios without deviating from the initial gas phase structure. However, when a 1:1 H-G ratio was tested experimentally, no Sb complex was observed which may indicate other factors contributing to binding like charge distributions and the HSAB principle. Thus, nitrogen and sulfur heteroatoms were substituted in the 18C6 ring structure.

\subsubsection{Effect of donor atoms and charge transfers}

Several strategies can be employed to understand electrostatic properties and factors that govern specific paths for guests not only within M-L complexes but with lone ligands. One strategy is molecular electrostatic potential maps (MESP or ESP) assist in the understanding of charge distributions of a molecule and predict the properties of an interaction site. This type of analysis is a powerful tool for identifying the possible interaction sites between a nucleophile and electrophile [67,68]. A MESP visually depicts several properties of structures with heteroatoms such as $\mathrm{O}$ (red), N (blue), and S (yellow) according to color. In Figure 6.5, different colors and hues extend gradually starting from red (electrophiles) to orange and eventually blue (nucleophiles). This not 
only depicts where electron-rich/deficient regions exist, but it also illustrates the boundaries of the electron density.
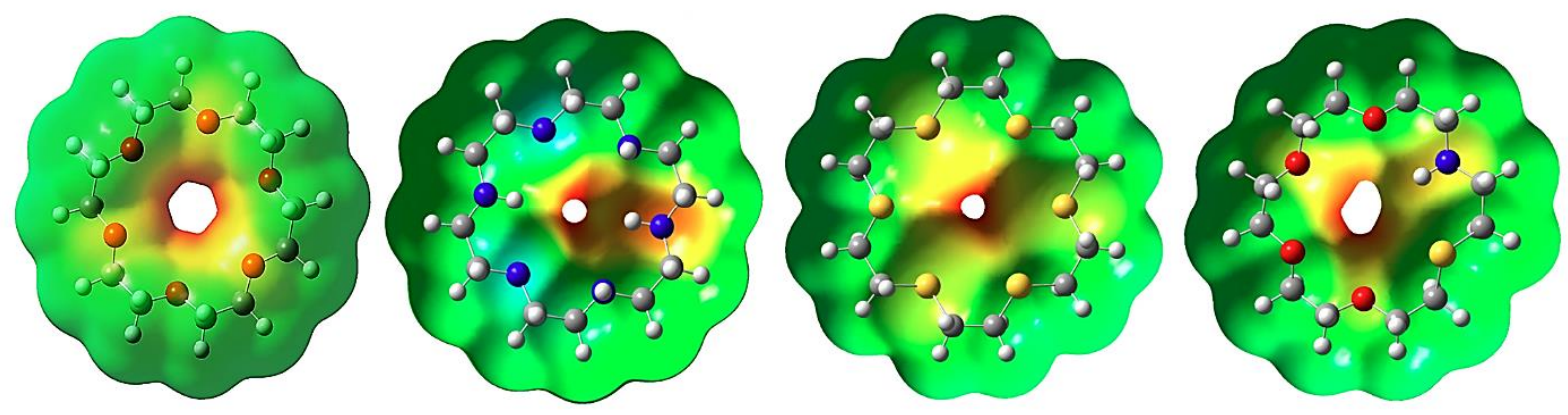

Figure 6.5: (Left to Right) Electrostatic distributions calculated for 18C6, hexacyclen, thia-crown, and partially substituted 18C6. Red regions represent electrophiles which contain an abundance of electrons (typically cause from lone pairs). Blue regions indicate nucleophiles where there may be a lower quantity of electrons.

The 18C6 ligand clearly depicts and directs guests towards a relatively large $(\sim 3.1 \AA)$ electron-rich inner cavity. For the hexacyclen macrocycle, there is an occurrence of more nucleophilic properties (blue region) as well as a smaller inner cavity $(\sim 2.1 \AA)$. This observation is primarily contributed to the nitrogen atoms maintaining their respective hydrogen which are free to reorient and interact for different interactions. For the thia-macrocycle, although a smaller cavity was observed, it is an electron-rich region originating from the lone pairs of electrons from the sulfur atoms. Finally, investigating the effects on mixtures of heteroatoms can affect the size and directionality of binding sites. For Figure 6.5, one nitrogen and one sulfur were substituted in the ring structure of the 18C6 macrocycle. While it retains a similarly sized hole compared to the 18C6, the nitrogen atom is pulling the electrons from the oxygen and sulfur atoms. This helps confirm the nature of nitrogen's nucleophilicity. This is ideal when trying to synthesize future capturing agents and concluding interactions.

An additional strategy to confirm the effects and contribution of various atoms in a ligand is using NBO analysis. This calculation assists in the investigation into interaction energies, $E^{2}$, 
of host-guest molecules (M-L complexes). In terms of the GSR studies using $\mathrm{Ba}, \mathrm{Pb}$, and $\mathrm{Sb}$, the contributing energies were attributed to the lone pair electrons of $\mathrm{O}$ atoms. These factors are depicted by $L P$ and $L P^{*}$ where the 1-center valence lone pair (LP1 and LP2) originate from the two lone pairs of each oxygen atom and the 1-center valence anti-bond lone pair on the metal, respectively.

Interestingly, Table 6.2 portrays a relationship between interactions energy and conformation changes amongst the major metal analytes found in GSR and 18C6. Here, the primary force contributing to interactions were the lone pairs $\mathrm{LP}^{*} \mathrm{sp}^{\mathrm{x}}$ orbitals of the crown's oxygen atoms (varying between 1.95 and 2.39) and the unoccupied 6s orbital of the cation of the oxygen atoms. To determine the atoms that contribute most to the transfer energies, the calculation of the second-order perturbation focuses on the energy between two species, "Unit 1" (host) to "Unit 2" (guest), which is outlined in Table S6.2. It can be shown that as the $E^{2}$ energy increases, the 18C6 ligand becomes more convoluted for different metal species. For the 18C6-Ba complex, a more linear conformation is observed while the $E^{2}$ energies from the contributing oxygen atoms are relatively low $(\sim 2.13)$. With the introduction of $\mathrm{Pb}$, the $E^{2}$ increased causing further structural change and encapsulation especially between $\mathrm{O} 1$ and $\mathrm{O} 22$, which are located on opposite positions of the ring. This observation could result from the value of the cation's electronegativity and diameter relationship $(\chi / \mathrm{d})$ being approximately $3 \mathrm{X}$ larger than the 18C6-Ba complex.

Table 6.2: NBO analysis of the contributing atoms to the charge transfer of ligand to metal guest. The driving mechanisms are identified by observing the energy contributions between "unit 1 to unit 2". 


\begin{tabular}{|c|c|c|c|}
\hline Structure Conformation & M-L complex & Donor NBO (i) $\rightarrow$ Acceptor NBO (j) & $\mathbf{E}^{(2)} \mathrm{kcal} / \mathrm{mol}$ \\
\hline & & $\mathrm{LP} 1 \mathrm{O} 1 \rightarrow \mathrm{LP} * 1 \mathrm{Ba} 43$ & 1.95 \\
\hline & & LP1 O8 $\rightarrow$ LP*1 $\mathrm{Ba} 43$ & 2.05 \\
\hline & & LP1 O15 $\rightarrow$ LP*1 Ba 43 & 2.03 \\
\hline & $18 \mathrm{C} 6-\mathrm{Ba}$ & $\mathrm{LP} 1 \mathrm{O} 22 \rightarrow \mathrm{LP} * 1 \mathrm{Ba} 43$ & 2.00 \\
\hline & & LP1 O29 $\rightarrow$ LP*1 Ba 43 & 2.36 \\
\hline 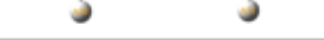 & & LP1 O36 $\rightarrow$ LP*1 Ba 43 & 2.39 \\
\hline & & $\mathrm{LP} 1 \mathrm{O} 1 \rightarrow \mathrm{LP} * 3 \mathrm{~Pb} 43$ & 15.76 \\
\hline & & $\mathrm{LP} 2 \mathrm{O} 8 \rightarrow \mathrm{LP} * 2 \mathrm{~Pb} 43$ & 7.45 \\
\hline & & LP2 $\mathrm{O} 15 \rightarrow \mathrm{LP} * 3 \mathrm{~Pb} 43$ & 7.23 \\
\hline & $18 \mathrm{C} 6-\mathrm{Pb}$ & $\mathrm{LP} 1 \mathrm{O} 22 \rightarrow \mathrm{LP} * 3 \mathrm{~Pb} 43$ & 15.42 \\
\hline & & $\mathrm{LP} 2 \mathrm{O} 29 \rightarrow \mathrm{LP} * 2 \mathrm{~Pb} 43$ & 6.53 \\
\hline & & $\mathrm{LP} 2 \mathrm{O} 36 \rightarrow \mathrm{LP} * 2 \mathrm{~Pb} 43$ & 6.62 \\
\hline & & LP1 O1 $\rightarrow$ LP*4 Sb 43 & 27.74 \\
\hline & & $\mathrm{LP} 2 \mathrm{O} 8 \rightarrow \mathrm{LP} * 4 \mathrm{Sb} 43$ & 14.36 \\
\hline & & $\mathrm{LP} 2 \mathrm{O} 15 \rightarrow \mathrm{LP} * 4 \mathrm{Sb} 43$ & 14.11 \\
\hline & $18 \mathrm{Cb}-\mathrm{Sb}$ & $\mathrm{LP} 1 \mathrm{O} 22 \rightarrow \mathrm{LP} * 4 \mathrm{Sb} 43$ & 27.81 \\
\hline & & $\mathrm{LP} 1 \mathrm{O} 29 \rightarrow \mathrm{LP} * 2 \mathrm{Sb} 43$ & 13.96 \\
\hline & & LP1 O36 $\rightarrow$ LP*4 Sb 43 & 13.85 \\
\hline
\end{tabular}

With the simulated 18C6-Sb complex data, the driving mechanism is the LP from two oxygen atoms $(\mathrm{O} 1$ and $\mathrm{O} 22)$ in which the $E^{2}$ energies are the highest at 27.74 and 27.81, respectively. Notably, by reorienting above the $\mathrm{Sb}$ atom, the energies from the remaining oxygen atoms increase (ranging from 13.85 to 14.36 ) as the distance to the metal decreases. These differences yield a large structural change from a relatively linear lone crown ether to a boat configuration. Even though 18C6 may have the capacity to bind to Sb, the large structural change may not be favorable as there is a small energy difference $(\Delta 5.09)$ from the 18C6-Sb complex (927.32 compared to -922.23 Hartrees) between the metal and lone crown ether macrocycle. Additionally, different combinations and substitutions of heteroatoms were added to the ring structure to observe their effects on binding to antimony and other metal ions for potentially a more selective response. All proposed macrocycles demonstrated the potential to interact with $\mathrm{Sb}$; however, there was no occurrence of this complex forming experimentally. Hence, these instances may be attributed to the HSAB principle where hard/soft atoms interact with their respective hard/soft species. 


\subsubsection{Metal guest influences on reactivity descriptors}

When understanding the association and conformation of M-L complexes, the properties of the metal species and ligands as well as the mechanisms of their reactions can be explained using the hard-soft acid-base (HSAB) concept. This theory stemmed from Pearson's qualitative concept where cations which are smaller in diameter and possess higher charge states are deemed "hard" acids or bases. These species are thus, less polarizable and typically form ionic bonds whereas, "softer" species form covalent bonds and are more polarizable, larger, and uncharged. However, "borderline" acids and bases exist where species possess intermediate properties such as high charge states and smaller cationic diameters (i.e., Sb). Thus, the strategy of utilizing noncovalent interactions becomes more difficult as there exists numerous macrocycles with various heteroatoms and functional groups.

To investigate potential macrocycles to encapsulate ions that observe HSAB properties, a more quantitative metric can be calculated to describe a more quantitative metric to describe interactions of hard and soft nucleophiles and electrophiles is to calculate the energies of HOMOs, LUMOs, and the energy gaps [66-68]. Conceptually, the narrower the HOMO-LUMO gap (low $\Delta \mathrm{E})$, the higher the chemical reactivity as it is favorable either to add an electron to a low-lying LUMO or to extract electrons from a high-lying HOMO [70,72]. Thus, the global reactivity descriptors of three fully substituted oxa-macrocycles were first calculated in both gas and solution phase, summarized in Table 6.3. 
Table 6.3: Fully substituted macrocycles with oxygen $\left(\mathrm{C}_{12} \mathrm{H}_{24} \mathrm{O}_{6}\right)$, nitrogen $\left(\mathrm{C}_{12} \mathrm{H}_{30} \mathrm{~N}_{6}\right)$, and sulfur $\left(\mathrm{C}_{12} \mathrm{H}_{24} \mathrm{~S}_{6}\right)$ atoms.

\begin{tabular}{|c|c|c|c|c|c|c|}
\hline Ligand & Phase & ELUMO $_{\text {Lu }}$ & Еномо & $\Delta \mathbf{E}$ & $\eta$ & $\mathbf{S}$ \\
\hline \multirow{4}{*}{$\mathrm{C}_{12} \mathrm{H}_{24} \mathrm{O}_{6}$} & Gas & 0.307 & -0.281 & 0.588 & 0.294 & 3.401 \\
\hline & $\mathrm{ACN}$ & 0.309 & -0.285 & 0.594 & 0.297 & 3.367 \\
\hline & $\mathrm{MeOH}$ & 0.296 & -0.299 & 0.595 & 0.298 & 3.361 \\
\hline & $\mathrm{H}_{2} \mathrm{O}$ & 0.294 & -0.300 & 0.594 & 0.297 & 3.367 \\
\hline \multirow{4}{*}{$\mathrm{C}_{12} \mathrm{H}_{30} \mathrm{~N}_{6}$} & Gas & 0.372 & -0.267 & 0.639 & 0.319 & 3.129 \\
\hline & $\mathrm{ACN}$ & 0.371 & -0.286 & 0.657 & 0.329 & 3.044 \\
\hline & $\mathrm{MeOH}$ & 0.370 & -0.288 & 0.658 & 0.329 & 3.039 \\
\hline & $\mathrm{H}_{2} \mathrm{O}$ & 0.367 & -0.294 & 0.661 & 0.331 & 3.025 \\
\hline \multirow{4}{*}{$\mathrm{C}_{12} \mathrm{H}_{24} \mathrm{~S}_{6}$} & Gas & 0.178 & -0.176 & 0.354 & 0.177 & 5.695 \\
\hline & $\mathrm{ACN}$ & 0.159 & -0.225 & 0.384 & 0.192 & 5.208 \\
\hline & $\mathrm{MeOH}$ & 0.160 & -0.225 & 0.385 & 0.193 & 5.194 \\
\hline & $\mathrm{H}_{2} \mathrm{O}$ & 0.160 & -0.226 & 0.386 & 0.193 & 5.181 \\
\hline
\end{tabular}

For the global hardness $(\eta)$, the fully substituted thia-macrocycle exhibited the smallest energy gap ( $\sim 0.38)$ compared to the fully substituted $o x a-$, and $a z a$-macrocycles $(\sim 0.58$ and $\sim 0.65$, respectively). Sulfur is the chemically softest atom due to its large atomic radius and, in turn, its valence electrons are the most polarizable. Both $N$ - and $O$-rich macrocycles were inversed to the thia-species and possessed similar responses in terms of these factors which can be contributed to their size and distance of their valence electron. The sulfur species also demonstrated the greatest affinity for the $\mathrm{Sb}$ species, followed by the $o x a$ - and $a z a$-macrocycles.

Relating to experimental observations, the newly formed M-L complex of the internal standard of $\mathrm{Tl}$ and the GSR analytes $(\mathrm{Ba}, \mathrm{Pb}$, and $\mathrm{Sb})$ had energy gaps of $6.405,6.865,5.486$, and $5.476 \mathrm{eV}$, respectively. In regard to the $18 \mathrm{C} 6$ host, the differences were $0.067,-0.393,0.986$, and $0.996 \mathrm{eV}$, respectively. Amongst the three polarizable solvents, the 18C6 energy gap did not differ significantly $(6.472 \mathrm{eV})$. With the addition of cations in solution, the energy gaps are altered which is primarily caused by the metal's orbitals extending the LUMO of the ligand (depicted in Figure 6.6). For electrospray ionization, more energy would be required to dissociate the highly reactive $\mathrm{Tl}^{+}$and $\mathrm{Ba}^{2+}$ as opposed to the $\mathrm{Pb}^{2+}$ and $\mathrm{Sb}^{3+}$ cationic species. 

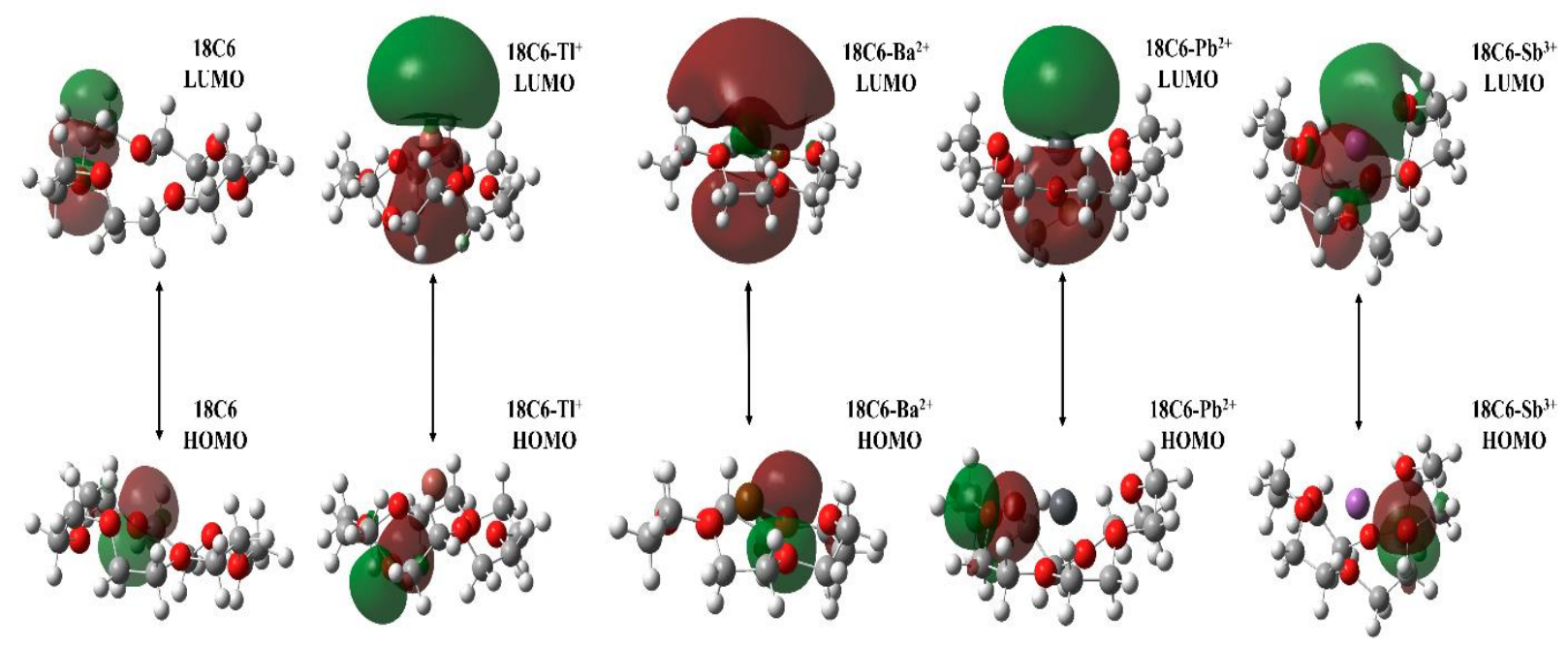

Figure 6.6: (Left to Right) HOMO and LUMO of lone $18 \mathrm{C} 6$ macrocycle with the addition of various metals and charge states $(+1,+2,+3)$. It can be shown that (for all instances) the HOMO mainly resided on the oxygen atoms whereas the LUMO exists on the metal cation.

The lone macrocycles orbitals were compared against the M-L complexes. As a metal cation was introduced, the traditional LUMO $p$-orbital structure can be observed for the guest analytes. Amongst these species, however, the LUMO density for barium seems to be vastly different. This may be attributed to the electron configuration of the $2+$ cation as electrons are lost from the $6 \mathrm{~s}$ orbital and thus, yielding a $[\mathrm{Xe}]$ configuration. From the NBO analysis, the barium species associates with noncovalent, electrostatic interactions via anti-bonding properties (LP*). It is important to note that as cations increase in size and possess more electrons in their outer shells (i.e., $p$ and $d$ ), the ability to distinguish between these orbitals becomes increasingly difficult as hybridization and overlap is possible. Thus, for the barium cation, this irregular orbital may be the product of this hybridization from the $\mathrm{sp}^{\mathrm{x}} \mathrm{d}^{\mathrm{y}}$ shells which has been recorded previously [71-73].

Amongst the studied cations, $\mathrm{Tl}^{+}$and $\mathrm{Ba}^{2+}$ demonstrated the largest displacement of the LUMO energy in the water due to the polarizability factor. Measuring from $\mathrm{O} 15$ and O36, the lone crown ether exhibited small radii cavity differences from gas phase $(3.06 \AA)$ in water $(2.95 \AA)$. For the $\mathrm{Tl}^{+}$and $\mathrm{Ba}^{2+}$ complexes, both radii were similar (2.95 $\AA$ ) but hovered above the cavity's center 
at approximately $2.39 \AA$ and $3.11 \AA$, respectively. Even though $\mathrm{Pb}^{2+}$ complex displayed similar LUMO energy displacement but was mitigated due to the crown ether's encapsulation which altered to $4.37 \AA$ for $\mathrm{O} 15$ and O36. Finally, $\mathrm{Sb}^{3+}$ cation exhibited the most interaction with the energies and the most distortion $(4.64 \AA$ ) of the $18 \mathrm{C} 6$ species. This suggests that the degree and magnitude of the LUMO energy contributes to the formation of "sandwich" structures as these cations have shown evidence to form these structures $[58,74]$.

For these analytes, the thia-macrocycles exhibited structural similarities for the $\mathrm{Pb}^{2+}$ and $\mathrm{Sb}^{3+}$ complexes; however, the displacement for the $\mathrm{Tl}^{+}$and $\mathrm{Ba}^{2+}$ were displacement father from the center of the cavity in solution phase as opposed to gas phase. This observation further illustrates the importance of monitoring these complexes in solution phase. Additionally, introducing counterions such as nitrates, hydroxides, and ammonium ions in solution may prove valuable to calculate since they are prevalent in a variety of mobile phases and may affect the formation of various macrocycles.

\subsection{Conclusions}

The use of computational methods has been utilized across various disciplines including chemo-sensing, extraction, and drug-design $[49,69]$. Recently, there has been a growing interest into utilization of these strategies for forensic purposes relating to drugs-of-abuse and explosives $[36,40,75]$. This work explored the possibility of utilizing this theoretical approach for the explanation of interactions and selectivity of a conglomeration typically found in GSR trace analysis [1,2]. Additionally, DFT was utilized to observe the main contributing factors for the inability to detect antimony within this particulate structure using ESI-MS/MS.

By using 18C6 as the starting structure, other simple macrocycles were simulated to elucidate the possible factors and interactions that take place in solution. In the presence of metal 
species such as $\mathrm{Ba}^{2+}$ or $\mathrm{Pb}^{2+}$, there is considerable displacement of these ions when exposed to common solvents such as methanol, acetonitrile, and water. Because of this, these metal ions are more prone to forming "sandwich" or 1:2 M-L structures. With the addition of $\mathrm{Tl}^{+}$(for purposes as an internal standard), the overall response was lowered suggesting $18 \mathrm{C} 6$ has selectivity of $\mathrm{Tl}>$ $\mathrm{Ba}>\mathrm{Pb}$ which correspond to DFT findings. From this study, findings indicate that crown ethers can complex with the metalloid Sb but to an unfavorable distortion of the base structure of the ligand.

It was also found, in both this study and others, that by altering the number of heteroatoms to either $\mathrm{N}$ or $\mathrm{S}$ atoms adjusts the selectivity for various metal cations [13,76-78]. These substitutions affect simple macrocycles by changing the inner cavity size and the lone pairs of electrons interacting with guest analytes. Furthermore, energy gaps exhibit important binding affinity trends with varying levels of chemical hardness amongst guests. This factor matters as scientists can manipulate and gain selectivity advantages when developing relatively inexpensive analytical tools such as point-of-care systems or even capillary columns.

One aspect that should be addressed in future studies is the effect of a counterion to binding affinity as well as the formation of "sandwich" structures. This is important due to the chemical composition of the conglomerate IGSR particles, and the procedure required to dissolve and expose these analytes to macrocycles in solution (i.e., heavy acid digestion) which has been observed in ESI (+) mode [2]. Additionally, using either an explicit or hybrid solvation model may introduce other factors into the competition between hosts and guests making it essential for MS/MS analysis to determine what complex(es) to target [43,44].

The overarching theoretical challenge in describing such flexible systems stems from the wealth of parameters required to describe their energetic, geometric, and chemical characteristics 
and the ease by which these parameters change. Electrospray ionization has allowed experimentalists to observe and calculate affinities through a myriad of factors which can then be compared to computational strategies. By observing positive relationships between experimental and theoretical data, scientists can further predict and design molecules to preserve physical resources. In this study, simple macrocyclic structures have the capacity to undergo high distortions albeit low energy changes when exposed to heavy metals whilst preserving their natural isotope distributions. These distortions relate to the strength of non-bonded interactions between the host and guest, which then teaches about the overall host-guest interaction energy. This work demonstrates the value of DFT to probe for valuable information and structures obtained from ESI-MS/MS experiments.

\section{Author Contributions}

The manuscript was written through the contributions of all authors. All authors have given approval to the final version of the manuscript.

\section{Conflicts of interest}

The authors declare that they have no known competing financial interests or personal relationships that could have influenced the work reported in this paper.

\section{Acknowledgments}

We want to acknowledge Dr. Kathleen Schwarz for troubleshooting and computational expertise for density functional calculations.

The authors acknowledge use of the supercomputing system Thorny Flat at West Virginia University (WVU), which is funded in part by the National Science Foundation EPSCoR Research Infrastructure Improvement Cooperative Agreement \#1003907, National Science Foundation 
Major Research Instrumentation Program (MRI) Award \#1726534, the state of West Virginia (WVEPSCoR via the Higher Education Policy Commission) and WVU.

This project is sponsored by Award No. \#2019-R2-CX-044 from the National Institute of Justice to West Virginia University. The opinions, findings, and conclusions are those of the authors and do not necessarily reflect those of the Department of Justice. 


\subsection{References for Chapter 6}

[1] W. Feeney, C. vander Pyl, S. Bell, T. Trejos, Trends in composition, collection, persistence, and analysis of IGSR and OGSR: A review, Forensic Chemistry. 19 (2020) 100250. https://doi.org/10.1016/j.forc.2020.100250.

[2] W. Feeney, K. Menking-Hoggatt, C. vander Pyl, C.E. Ott, S. Bell, L. Arroyo, T. Trejos, Detection of Organic and Inorganic Gunshot Residues from Hands using Complexing Agents and LC-MS/MS, Analytical Methods. (2021). https://doi.org/10.1039/d1ay00778e.

[3] M.E. Sears, Chelation: Harnessing and enhancing heavy metal detoxification - A review, The Scientific World Journal. 2013 (2013). https://doi.org/10.1155/2013/219840.

[4] B.E. Igiri, S.I.R. Okoduwa, G.O. Idoko, E.P. Akabuogu, A.O. Adeyi, I.K. Ejiogu, Toxicity and Bioremediation of Heavy Metals Contaminated Ecosystem from Tannery Wastewater: A Review, Journal of Toxicology. (n.d.). https://doi.org/10.1155/2018/2568038. [5] C. Sarzanini, E. Mentasti, Determination and speciation of metals by liquid chromatography, Journal of Chromatography A. 789 (1997) 301-321. https://doi.org/10.1016/S0021-9673(97)00988-6.

[6] M.J. Kosnett, The Role of Chelation in the Treatment of Arsenic and Mercury Poisoning, Journal of Medical Toxicology. 9 (2013) 347-354. https://doi.org/10.1007/s13181-013-0344-5.

[7] A. Ciesielski, P. Samori, Supramolecular Approaches to Graphene: From Self-Assembly to Molecule-Assisted Liquid-Phase Exfoliation, Advanced Materials. 28 (2016) 6030-6051. https://doi.org/10.1002/adma.201505371.

[8] I. v. Kolesnichenko, E. v. Anslyn, Practical applications of supramolecular chemistry, Chemical Society Reviews. 46 (2017) 2385-2390. https://doi.org/10.1039/c7cs00078b. 
[9] J.J. Guo, J. Lee, C.I. Contescu, N.C. Gallego, S.T. Pantelides, S.J. Pennycook, B.A.

Moyer, M.F. Chisholm, Crown ethers in graphene, Nature Communications. 5 (2014) 6. https://doi.org/10.1038/ncomms6389.

[10] S. Kafashi, M. Yaftian, A. Zamani, Binding Ability of Crown Ethers Towards Pb(II) Ions in Binary Water/Organic Solvents Using Solvent Extraction Method, Journal of Solution Chemistry. 44 (2015) 1798-1811. https://doi.org/10.1007/s10953-015-0378-1.

[11] S. Bell, W. Feeney, Single shot, single sample, single instrument detection of IGSR and OGSR using LC/MS/MS, Forensic Science International. 299 (2019) 215-222. https://doi.org/10.1016/j.forsciint.2019.04.002.

[12] Y. Inokuchi, O. v. Boyarkin, R. Kusaka, T. Haino, T. Ebata, T.R. Rizzo, UV and IR spectroscopic studies of cold alkali metal ion-crown ether complexes in the gas phase, Journal of the American Chemical Society. 133 (2011) 12256-12263. https://doi.org/10.1021/ja2046205.

[13] Z. Zhao, H. Tian, M. Zhang, Y. Yang, H. Zhang, Molecular design of macrocyclic compounds for complete removal of thallium(I) from wastewater, Environmental Science and Pollution Research. 25 (2018) 34550-34558. https://doi.org/10.1007/s11356-018-3393-0. [14] X. Du, H. Hao, A. Qin, B.Z. Tang, Highly sensitive chemosensor for detection of methamphetamine by the combination of AIE luminogen and cucurbit[7]uril, Dyes and Pigments. 180 (2020) 108413. https://doi.org/https://doi.org/10.1016/j.dyepig.2020.108413.

[15] F. Ma, X. Zheng, J. Xie, Z. Li, Binding properties of cucurbit[7]uril to neutral and protonated amino acids: A theoretical study, International Journal of Quantum Chemistry. (n.d.). https://doi.org/10.1002/qua.26491. 
[16] L. Barrientos, S. Miranda-Rojas, F. Mendizabal, Noncovalent interactions in inorganic supramolecular chemistry based in heavy metals. Quantum chemistry point of view, International Journal of Quantum Chemistry. 119 (2019). https://doi.org/10.1002/qua.25675. [17] H.J. Schneider, A.K. Yatsimirsky, Selectivity in supramolecular host-guest complexes, Chemical Society Reviews. 37 (2008) 263-277. https://doi.org/10.1039/b612543n.

[18] S.M. Blair, E.C. Kempen, J.S. Brodbelt, Determination of binding selectivities in hostguest complexation by electrospray/quadrupole ion trap mass spectrometry, Journal of the American Society for Mass Spectrometry. 9 (1998) 1049-1059. https://doi.org/10.1016/s1044$\underline{0305(98) 00082-8 .}$

[19] Y. Tao, R.R. Julian, Factors that influence competitive intermolecular solvation of protonated groups in peptides and proteins in the gas phase, Journal of the American Society for Mass Spectrometry. 24 (2013) 1634-1640. https://doi.org/10.1007/s13361-013-0684-z.

[20] V. Rudiger, H.J. Schneider, V.P. Solov'ev, V.P. Kazachenko, O.A. Raevsky, Crown ether-ammonium complexes: Binding mechanisms and solvent effects, European Journal of Organic Chemistry. (1999) 1847-1856. \%3CGo.

[21] C.L. Sherman, J.S. Brodbelt, A.P. Marchand, B. Poola, Electrospray ionization mass spectrometric detection of self-assembly of a crown ether complex directed by $\pi$-stacking interactions, Journal of the American Society for Mass Spectrometry. 16 (2005) 1162-1171. https://doi.org/10.1016/j.jasms.2005.03.014.

[22] S.M. Blair, J.S. Brodbelt, A.P. Marchand, K.A. Kumar, H.-S. Chong, Evaluation of Binding Selectivities of Caged Crown Ligands toward Heavy Metals by Electrospray Ionization/Quadrupole Ion Trap Mass Spectrometry, Analytical Chemistry. 72 (2000) 24332445. https://doi.org/10.1021/ac991125t. 
[23] A. McKenzie-Coe, S. Bell, F. Fernandez-Lima, Detection of firearm discharge residue from skin swabs using trapped ion mobility spectrometry coupled to mass spectrometry, Analytical Methods. 10 (2018) 4219-4224. https://doi.org/10.1039/c8ay00658j.

[24] A.B. Wijeratne, S.H. Yang, D.W. Armstrong, K.A. Schug, Solvent Molecules Undergo Homolytic Cleavage and Radical Recombination Processes during Negative-Mode Electrospray Ionization: Adduct Formation with Antimony(III)-Tartrate Dianion, Analytical Chemistry. 82 (2010) 5141-5146. https://doi.org/10.1021/ac1003344.

[25] A.B. Wijeratne, S.E. Spencer, J. Gracia, D.W. Armstrong, K.A. Schug, Antimony(III)-D, L-Tartrates Exhibit Proton-Assisted Enantioselective Binding in Solution and in the Gas Phase, Journal of the American Society for Mass Spectrometry. 20 (2009) 2100-2105. https://doi.org/10.1016/j.jasms.2009.07.011.

[26] A.B. Wijeratne, J. Gracia, S.H. Yang, P. Kroll, D.W. Armstrong, K.A. Schug, New structural insight for antimony(III)-tartrate, Inorganic Chemistry Communications. 13 (2010) 1504-1508. https://doi.org/10.1016/J.INOCHE.2010.08.028.

[27] A.B. Wijeratne, K.A. Schug, Molecular recognition properties of tartrates and metaltartrates in solution and gas phase, Journal of Separation Science. 32 (2009) 1537-1547. https://doi.org/10.1002/jssc.200900064.

[28] N. Islam, S.S. Chimni, Binding and selectivity of phenazino-18-crown-6-ether with alkali, alkaline earth and toxic metal species: A DFT study, Journal of Molecular Structure. (2017). https://doi.org/10.1016/j.molstruc.2016.10.100.

[29] Z. Jing, G. Wang, Y. Zhou, D. Pang, F. Zhu, H. Liu, Selectivity of 18-crown-6 ether to alkali ions by density functional theory and molecular dynamics simulation, Journal of Molecular Liquids. 311 (2020). https://doi.org/10.1016/j.molliq.2020.113305. 
[30] K.J. Hintze, A. Lützen, T. Bredow, Structure and stability of supramolecular crown ether complexes, Journal of Computational Chemistry. 36 (2015) 1467-1472.

https://doi.org/10.1002/jcc.23950.

[31] S. Hadisaputra, H.D. Pranowo, R. Armunanto, Extraction of strontium(ii) by crown ether: Insights from density functional calculation, Indonesian Journal of Chemistry. 12 (2012) 207216. https://doi.org/10.22146/ijc.21332.

[32] N.N. Karaush, G. v. Baryshnikov, V.A. Minaeva, B.F. Minaev, A DFT and QTAIM study of the novel d-block metal complexes with tetraoxa[8]circulene-based ligands, New Journal of Chemistry. 39 (2015) 7815-7821. https://doi.org/10.1039/c5nj01255d.

[33] R. Behjatmanesh-Ardakani, F. Pourroustaei-Ardakani, M. Taghdiri, Z.M. Kotena, DFTB3LYP study of interactions between host biphenyl-1-aza-18-crown-6 ether derivatives and guest Cd2+: NBO, NEDA, and QTAIM analyses, Journal of Molecular Modeling. 22 (2016). https://doi.org/10.1007/s00894-016-3012-2.

[34] K. Reuter, M.R. Buchner, G. Thiele, C. von Hänisch, Stable Alkali-Metal Complexes of Hybrid Disila-Crown Ethers, Inorganic Chemistry. 55 (2016) 4441-4447.

https://doi.org/10.1021/acs.inorgchem.6b00267.

[35] D.M. Zuckerman, Statistical physics of biomolecules: An introduction, Statistical Physics of Biomolecules: An Introduction. (2010) 1-325. https://doi.org/10.1201/b18849.

[36] M. Jaidann, S. Roy, H. Abou-Rachid, L.S. Lussier, A DFT theoretical study of heats of formation and detonation properties of nitrogen-rich explosives, Journal of Hazardous Materials. 176 (2010) 165-173. https://doi.org/10.1016/j.jhazmat.2009.10.132.

[37] S.J.J.J.J. Mary, M.U.M. Siddique, S. Pradhan, V. Jayaprakash, C. James, Quantum chemical insight into molecular structure, NBO analysis of the hydrogen-bonded interactions, 
spectroscopic (FT-IR, FT-Raman), drug likeness and molecular docking of the novel anti COVID-19 molecule 2-[(4,6-diaminopyrimidin-2-yl)sulfanyl]-N-(4-fluo, Spectrochimica Acta Part A: Molecular and Biomolecular Spectroscopy. 244 (2021) 118825. https://doi.org/10.1016/j.saa.2020.118825.

[38] E. Bahojb Noruzi, M. Molaparast, M. Zarei, B. Shaabani, Z. Kariminezhad, B. Ebadi, V. Shafiei-Irannejad, M. Rahimi, J. Pietrasik, E.B. Noruzi, M. Molaparast, M. Zarei, B. Shaabani, Z. Kariminezhad, B. Ebadi, V. Shafiei-Irannejad, M. Rahimi, J. Pietrasik, Parasulfonatocalix[n]arene-based biomaterials: Recent progress in pharmaceutical and biological applications, European Journal of Medicinal Chemistry. 190 (2020). https://doi.org/10.1016/j.ejmech.2020.112121.

[39] L. Isaacs, Stimuli responsive systems constructed using cucurbit[ $\mathrm{n}$ ]uril-type molecular containers, Accounts of Chemical Research. 47 (2014) 2052-2062. https://doi.org/10.1021/ar500075g.

[40] M. Vujović, V. Ragavendran, B. Arsić, E. Kostić, M. Mladenović, DFT calculations as an efficient tool for prediction of Raman and infra-red spectra and activities of newly synthesized cathinones, Open Chemistry. 18 (2020) 185-195. https://doi.org/10.1515/chem-2020-0021. [41] A.A. Al-Kahtani, N.A. Al-Jallal, A.A. El-Azhary, Conformational and vibrational analysis of 18-crown-6-alkali metal cation complexes, Spectrochimica Acta - Part A: Molecular and Biomolecular Spectroscopy. 132 (2014) 70-83. https://doi.org/10.1016/j.saa.2014.04.170. [42] Y. Zhang, X.Y. Wang, B.H. Luo, Y. Xia, DFT study of crown ether-bridged Z-stilbenes and their complexes with alkali metal cations, Journal of Organometallic Chemistry. 699 (2012) 31-38. https://doi.org/10.1016/j.jorganchem.2011.10.032. 
[43] J. Zhang, H. Zhang, T. Wu, Q. Wang, D. van der Spoel, Comparison of Implicit and Explicit Solvent Models for the Calculation of Solvation Free Energy in Organic Solvents, (2017). https://doi.org/10.1021/acs.jctc.7b00169.

[44] M. Wang, Y. Mei, U. Ryde, Host-Guest Relative Binding Affinities at DensityFunctional Theory Level from Semiempirical Molecular Dynamics Simulations, Journal of Chemical Theory and Computation. 15 (2019) 2659-2671. https://doi.org/10.1021/acs.jctc.8b01280.

[45] N. Kircheva, S. Dobrev, L. Dasheva, I. Koleva, V. Nikolova, S. Angelova, T. Dudev, B. Supramolecular, Complexation of biologically essential (mono- And divalent) metal cations to cucurbiturils: a DFT/SMD evaluation of the key factors governing the host-guest recognition, RSC Advances. 10 (2020) 28139-28147. https://doi.org/10.1039/d0ra04387g.

[46] E.S.H. Gwee, Z.L. Seeger, D.R.T. Appadoo, B.R. Wood, E.I. Izgorodina, Influence of DFT Functionals and Solvation Models on the Prediction of Far-Infrared Spectra of Pt-Based Anticancer Drugs: Why Do Different Complexes Require Different Levels of Theory?, ACS Omega. 4 (2019) 5254-5269. https://doi.org/10.1021/acsomega.8b03455.

[47] R. Behjatmanesh-Ardakani, DFT-B3LYP and SMD study on the interactions between aza-, diaza-, and triaza-12-crown-4 (A n-12-crown-4, $\mathrm{n}=1,2,3)$ with $\mathrm{Na}+$ in the gas phase and acetonitrile solution, Structural Chemistry. 25 (2014) 919-929. https://doi.org/10.1007/s11224013-0366-2.

[48] K.A. Baseden, J.W. Tye, Introduction to density functional theory: Calculations by hand on the helium atom, Journal of Chemical Education. 91 (2014) 2116-2123. https://doi.org/10.1021/ed5004788. 
[49] B. Honarparvar, S. Kanchi, K. Bisetty, Theoretical insights into the competitive metal bioaffinity of lactoferrin as a metal ion carrier: A DFT study, New Journal of Chemistry. 43 (2019) 16374-16384. https://doi.org/10.1039/c9nj03786a.

[50] H. Xu, D.C. Xu, Y. Wang, Natural indices for the chemical hardness/softness of metal cations and ligands, ACS Omega. 2 (2017) 7185-7193.

https://doi.org/10.1021/acsomega.7b01039.

[51] R.P. Gangadharana, S.S. Krishnanb, Natural Bond Orbital (NBO) population analysis of 1-azanapthalene-8-ol, Acta Physica Polonica A. 125 (2014) 18-22.

https://doi.org/10.12693/APhysPolA.125.18.

[52] W. Li, B. Lu, F. Chen, F. Yang, Z. Wang, Host-guest complex of cypermethrin with $\beta$ cyclodextrin: A spectroscopy and theoretical investigation, Journal of Molecular Structure. 990 (2011) 244-252. https://doi.org/10.1016/j.molstruc.2011.01.053.

[53] F. Weinhold, C.R. Landis, E.D. Glendening, What is NBO analysis and how is it useful?, International Reviews in Physical Chemistry. 35 (2016) 399-440. https://doi.org/10.1080/0144235X.2016.1192262.

[54] S. Hirata, X. He, M.R. Hermes, S.Y. Willow, Second-order many-body perturbation theory: An eternal frontier, Journal of Physical Chemistry A. 118 (2014) 655-672. https://doi.org/10.1021/jp410587b.

[55] N. Yasarawan, K. Thipyapong, V. Ruangpornvisuti, Substituent effect on the protonrelated phenomena and chelation behavior of hydroxypicolinic compounds: A DFT investigation, Structural Chemistry. 27 (2016) 505-524. https://doi.org/10.1007/s11224-015$\underline{0579-7 .}$ 
[56] A. Hassan, M. Mahmoud, B.S. Bageri, M.S. Aljawad, M.S. Kamal, A.A. Barri, I.A.

Hussein, Applications of chelating agents in the upstream oil and gas industry: A review, Energy and Fuels. 34 (2020) 15593-15613. https://doi.org/10.1021/acs.energyfuels.0c03279.

[57] H.J. Schneider, Limitations and Extensions of the Lock-and-Key Principle: Differences between Gas State, Solution and Solid State Structures, International Journal of Molecular Sciences. 16 (2015) 6694-6717. https://doi.org/10.3390/ijms16046694.

[58] H.R. Yu, J.Q. Hu, X.H. Lu, X.J. Ju, Z. Liu, R. Xie, W. Wang, L.Y. Chu, Insights into the effects of 2:1 "sandwich-type" crown-ether/metal-ion complexes in responsive host - guest systems, Journal of Physical Chemistry B. 119 (2015) 1696-1705. https://doi.org/10.1021/jp5079423.

[59] A. Kruve, K. Kaupmees, Adduct Formation in ESI/MS by Mobile Phase Additives, Journal of the American Society for Mass Spectrometry. 28 (2017) 887-894. https://doi.org/10.1007/s13361-017-1626-y.

[60] L. Konermann, H. Metwally, Q. Duez, I. Peters, Charging and supercharging of proteins for mass spectrometry: Recent insights into the mechanisms of electrospray ionization, Analyst. 144 (2019) 6157-6171. https://doi.org/10.1039/c9an01201j.

[61] E.M. Panczyk, J.D. Gilbert, G.S. Jagdale, A.Q. Stiving, L.A. Baker, V.H. Wysocki, Ion Mobility and Surface Collisions: Submicrometer Capillaries Can Produce Native-like Protein Complexes, Analytical Chemistry. 92 (2020) 2460-2467.

https://doi.org/10.1021/acs.analchem.9b03666.

[62] C.L. Sherman, J.S. Brodbelt, Partitioning Model for Competitive Host-Guest

Complexation in ESI-MS, Analytical Chemistry. 77 (2005) 2512-2523.

https://doi.org/10.1021/ac048518z. 
[63] C.J. Pedersen, The Discovery of Crown Ethers, Science. 241 (1988) 536-540. http://www.jstor.org/stable/1701735.

[64] M.R. Sambrook, J.C. Vincent, J.A. Ede, I.A. Gass, P.J. Cragg, Experimental and computational study of the inclusion complexes of beta-cyclodextrin with the chemical warfare agent soman (GD) and commonly used simulants, Rsc Advances. 7 (2017) 38069-38076. https://doi.org/10.1039/c7ra03328a.

[65] A.I. Lazar, F. Biedermann, K.R. Mustafina, K.I. Assaf, A. Hennig, W.M. Nau, Nanomolar Binding of Steroids to Cucurbit[n]urils: Selectivity and Applications, Journal of the American Chemical Society. 138 (2016) 13022-13029. https://doi.org/10.1021/jacs.6b07655.

[66] A. v. Marenich, C.J. Cramer, D.G. Truhlar, Universal solvation model based on solute electron density and on a continuum model of the solvent defined by the bulk dielectric constant and atomic surface tensions, Journal of Physical Chemistry B. 113 (2009) 6378-6396. https://doi.org/10.1021/jp810292n.

[67] M.D. Esrafili, Nitrogen-doped (6,0) carbon nanotubes: A comparative DFT study based on surface reactivity descriptors, Computational and Theoretical Chemistry. 1015 (2013) 1-7. https://doi.org/10.1016/j.comptc.2013.04.003.

[68] M.D. Esrafili, F. Mohammadian-Sabet, An ab initio study on chalcogen-chalcogen bond interactions in cyclic (SHX)3 complexes $(\mathrm{X}=\mathrm{F}, \mathrm{Cl}, \mathrm{CN}, \mathrm{NC}, \mathrm{CCH}, \mathrm{OH}, \mathrm{OCH} 3, \mathrm{NH} 2)$, Chemical Physics Letters. 628 (2015) 71-75. https://doi.org/10.1016/j.cplett.2015.04.013. [69] M. Ramalingam, V. Sethuraman, N. Sundaraganesan, Molecular structure, vibrational spectroscopic, first order hyperpolarizability and HOMO-LUMO studies of 7-amino-8-oxo-3vinyl-5-thia-1- azabicyclo[4.2.0]oct-2-ene-2-carboxylic acid, Spectrochimica Acta - Part A: 
Molecular and Biomolecular Spectroscopy. 78 (2011) 660-669.

https://doi.org/10.1016/j.saa.2010.11.027.

[70] S.K. Seth, I. Saha, C. Estarellas, A. Frontera, T. Kar, S. Mukhopadhyay, Supramolecular self-assembly of M-IDA complexes involving lone-pair $\cdots \varphi$ Interactions: Crystal structures, hirshfeld surface analysis, and DFT calculations [H2IDA = iminodiacetic acid, $\mathrm{M}=\mathrm{Cu}(\mathrm{II})$, Ni(II)], Crystal Growth and Design. 11 (2011) 3250-3265. https://doi.org/10.1021/cg200506q.

[71] X. Wu, L. Zhao, D. Jiang, I. Fernández, R. Berger, M. Zhou, G. Frenking, Barium as Honorary Transition Metal in Action: Experimental and Theoretical Study of $\mathrm{Ba}(\mathrm{CO})+$ and Ba(CO)-, Angewandte Chemie - International Edition. 57 (2018) 3974-3980. https://doi.org/10.1002/anie.201713002.

[72] K.L. Woon, W.S. Wong, N. Chanlek, H. Nakajima, S. Tunmee, V.S. Lee, A. Ariffin, P. Songsiriritthigul, Work function modification of PEDOT:PSS by mixing with barium acetylacetonate, RSC Advances. 10 (2020) 17673-17680. https://doi.org/10.1039/d0ra02575e. [73] R. Oubouaza, M. Benson, J. Wojciechowski, S. Chtita, M. Tridane, S. Belaaouad, Synthesis, crystal structure, vibrational study and DFT computation of barium dihydrogenomonophosphate $\mathrm{Ba}(\mathrm{H} 2 \mathrm{PO} 4) 2$, Biointerface Research in Applied Chemistry. 12 (2022) 1120-1133. https://doi.org/10.33263/BRIAC121.11201133.

[74] K.I. Tugashov, D.A. Gribanyov, F.M. Dolgushin, A.F. Smol'yakov, A.S. Peregudov, M.K. Minacheva, I.A. Tikhonova, V.B. Shur, Coordination Chemistry of Anticrowns. Synthesis and Structures of Double-Decker Sandwich Complexes of the Three-Mercury Anticrown (oC6F4Hg)(3) with Halide Anions Containing and Not Containing Coordinated Dibromomethane Molecules, Organometallics. 35 (2016) 2197-2206.

https://doi.org/10.1021/acs.organomet.6b00231. 
[75] J. Tyler Davidson, E.L. Piacentino, Z.J. Sasiene, Y. Abiedalla, J. DeRuiter, C.R. Clark, G. Berden, J. Oomens, V. Ryzhov, G.P. Jackson, Identification of novel fragmentation pathways and fragment ion structures in the tandem mass spectra of protonated synthetic cathinones, Forensic Chemistry. 19 (2020) 100245. https://doi.org/10.1016/j.forc.2020.100245.

[76] M. Frańska, A. Michalak, Gas-phase relative stabilities of Tl+-crown ether complexes and Rb+-crown ether complexes, European Journal of Mass Spectrometry. 24 (2018) 279-288. https://doi.org/10.1177/1469066717752794.

[77] S.M. Williams, J.S. Brodbelt, A.P. Marchand, D. Cal, K. Mlinaric-Majerski, Metal complexation of thiacrown ether macrocycles by electrospray ionization mass spectrometry, Analytical Chemistry. 74 (2002) 4423-4433. https://doi.org/10.1021/ac011227v.

[78] V. Rüdiger, H.-J. Schneider, V.P. Solov’ev, V.P. Kazachenko, O.A. Raevsky, Crown Ether-Ammonium Complexes: Binding Mechanisms and Solvent Effects, European Journal of Organic Chemistry. 1999 (1999) 1847-1856. https://doi.org/10.1002/(sici)1099-

0690(199908)1999:8<1847::aid-ejoc1847>3.3.co;2-h. 


\section{CHAPTER 7}

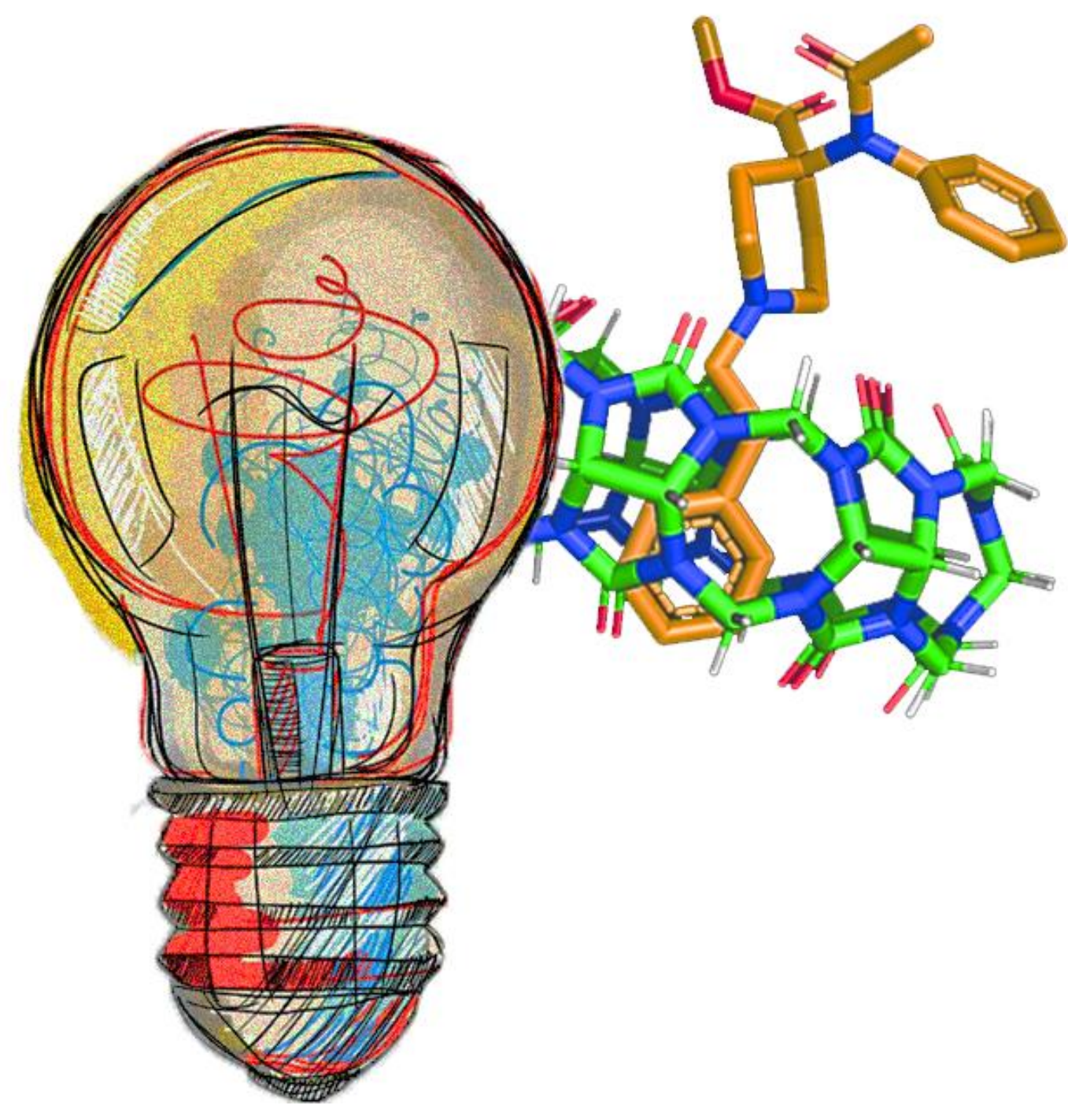

Overall Conclusion and Future Directions 


\subsection{Overall Conclusion and Future Directions}

This study met our overall goal of enhancing the reliability of GSR evidence through the development of rapid analytical tools that provided an increased body of knowledge on the chemical composition of modern ammunition residues and a novel interpretation framework for the quantitative assessment of the evidential value of GSR. We anticipate that the methodologies and the IGSR/OGSR population dataset will become accessible to forensic practitioners to complement and modernize current practice. The main findings and achievements of this study are discussed in the following sections.

\subsubsection{Objective 1: Review of the current literature associated with GSR}

The literature review condenses relevant literature within the past 20 years associated with gunshot residue's collection and analysis. Most investigations primarily focused on the IGSR particulate information using SEM-EDS instrumentation and reported data on particle counts and composition. The forensic community benefits from international standard practices that are broadly implemented and studied. Also, a guideline for validation of GSR examinations by SEMEDS was recently published, assisting further the community to stringent quality controls. The research groups that did investigate OGSR compound information utilized various instruments ranging from GC/MS, LC-MS/MS, IMS, Raman, and TOF-MS. The status of OGSR detection is still in research stages and therefore the emerging tools reported in the literature have not been widely adopted in the field. Consensus-based method are not yet implemented for combined OGSR to IGSR examinations, but it is a leading research topic in this discipline. Because of this, practitioners and researchers have just scraped the surface of the information that can be obtained from utilizing combined OGSR and IGSR information from a single sample. 
The review paper (Chapter 3) highlighted some of those existing gaps and summarized the scientific foundations and current state for the deposition, persistence, and collection of organic and inorganic gunshot residue. One of the outcomes of this manuscript was to provide an extensive summary of the main OGSR and IGSR components reported in the literature and a comparative analysis of the limitations and capabilities of methods of collection, preservation, and examination of GSR.

Regardless of numerous studies reporting transfer and persistence of IGSR and OGSR, this topic remains challenging, and several gaps of knowledge still exist. Advances and adoption of novel analytical methods, and an increased body of knowledge on background occurrence and transfer mechanisms will lead the field to more encompassing interpretation models.

The research conducted in this dissertation is anticipated to contribute to a needed knowledge in this field. First, the development of novel methods of IGSR/OGSR detection (Objective 2) and their application to population datasets (Objective 3) and simulation computational models (Objective 4) increases our understanding of the chemistry and the mechanisms of prevalence and persistence of GSR. Second, this study proposed using machine learning algorithms to assist in the probabilistic interpretation of the evidence (objective 3), opening modern and more encompassing venues to assist the trier of fact.

\subsubsection{Objective 2: Validation and evaluation of a single instrument for sequential analysis of}

\section{GSR components}

In Chapter 4, an LC-MS/MS method was developed, optimized, and validated for dual detection of IGSR and OGSR from a single sample, using a single instrument. Because of the properties of host-guest chemistry are less known to the forensics community, further confirmation was required to demonstrate the identification and characterization of the IGSR analytes when 
associated with the crown ethers. Hence, the high-resolution orbitrap was implemented to identify the products and natural abundances of the metal ions.

The analytical figures of merit (LOD, LOQ, LDR, intra- and interday variabilities, selectivity) and the performance rates derived from ground truth specimens from shooters and nonshooters demonstrated the method fit for purpose. Also, the efficiency of two collection and extraction protocols were compared for this study, tesa Tack® and the ASTM carbon adhesive recommended for IGSR. Extractions were performed side-by-side and the percent recoveries were analyzed by ANOVA. The OGSR compounds were adequately analyzed via LC-MS/MS after the organic extraction step. However, because of the crown ether mechanisms, the recovery of IGSR was cross-evaluated by ICP-MS with a more exhaustive digestion method. Hence, an ICP-MS methodology was used to evaluate the concentrations of the metal ions using the different extraction procedures. The results indicate the carbon adhesive provided superior performance while conveniently being compatible with current collection protocols for SEM-EDS.

A set of authentic samples were extracted and analyzed from the carbon adhesives using the LC-MS/MS method. Because this technique sequentially analyzes IGSR and OGSR, a conservative criterion was established as a first step, following a categorical approach similar to the ASTM E1588-20. This criterion indicated that a sample was positive if at least three GSR components were present above the cut-off (one OGSR and one IGSR, and vice versa.)

Furthermore, by noting the lack of investigations using screening methodologies in GSRrelated casework, a workflow was developed to incorporate LIBS and electrochemical methods before LC-MS/MS confirmatory analysis. Samples from authentic shootings and post-shooting activities were evaluated to test value of combining information. In both situations, the knowledge of a sample increased after incorporating both OGSR and IGSR information. LC-MS/MS provided 
superior performance and was able to detect both inorganic and organic components from the sample after quick screening with LIBS/EC, demonstrating it can be used as a stand-alone method and as a confirmatory method when large volume of items in a case may require some triage screening alternatives.

In this work, it was concluded that environmental factors greatly affect the recovery efficiency, and thus, the overall reporting of GSR. This work also illustrated that practitioners and crime laboratories do not need to choose between collecting and analyzing OGSR and IGSR information, providing a breakthrough alternative approach for this comprehensive examination of organic and inorganic residues.

\subsubsection{Objective 3: Investigation of the presence and prevalence of GSR in West Virginia within different populations}

The goal of this work was to investigate the prevalence and presence of GSR in the population of West Virginia using the validated LC-MS/MS method. Because of the presence of potential false-positive and false-negative samples, the ability to differentiate and classify among casework specimens are critical and challenging for forensic scientists. Additionally, the presence and identification of GSR are reliant on the nature of the constituent and the surrounding environmental conditions. The OGSR compounds are less prone to secondary transfer due to their lipophilic nature and ability to absorb into the epidermal layer of the skin. The IGSR particulates adhere to the top layer of the skin and clothing but are more susceptible to secondary and even tertiary transfer when physically disrupted by outside forces. Because of these key features, individuals from five separate populations were sampled and analyzed by the LC-MS/MS.

Over 400 individuals were stubbed from different locations surrounding Morgantown and included background individuals with no association to firearms and individuals who work in 
professions such as police-officers, farmers, and mechanics that can contain GSR and lead to falsepositive identifications. The other sets consisted of bystanders that were near a shooting event, post-shooting activities, and authentic shooters. The authentic shooters and low-risk sample sets were used as upper and lower limits, respectively, to compare the three remaining population sets. Exploratory analysis was first utilized to identify any trends in the subgroups. It was concluded that OGSR was mostly present after a discharge event (shooting and post-shooting activities) whereas signals for the IGSR analytes were present in all populations but in varying relative concentrations. Most importantly, the combined occurrence of IGSR/OGSR was only observed in shooters sets and not in background samples, demonstrating the relevance of using the combined compound strategies for GSR interpretation.

Data was further evaluated by machine learning algorithms such as neural networks and logistic regression models. Here, the probabilities of each population were calculated and utilized to construct likelihood ratios for more comprehensive evaluations and assessment of the weight of the evidence. It was found that the neural network algorithm produced low error rates $(<5 \%)$ for population classification. Moreover, the use of LR demonstrated a valuable metric of the probative strength of the evidence on the various populations.

7.1.4. Objective 4: Investigations into host-guest interactions with metal ions using DFT for applications using mass spectrometry

Another goal of this work was to investigate the underlying factors influencing the inability of crown ether to interact with an important analyte found in GSR, antimony. By utilizing density functional theory, a quantum mechanical approach to assist in solving the famous Schrodinger equation, scientists can relate and more importantly visualize the effects of changing parameters. 
Hence the overarching goal of this study was to highlight the main effects that may increase or decrease the interactions for specific metallic ions, both in gas and solution phases. Ultimately, 18C6 and other simple macrocycles were simulated to elucidate the possible factors and interactions that take place in solution. In this study, we exposed the possible variations of not only the conformers of $18 \mathrm{C} 6$, but also with varying numbers of heteroatom substitutions and positioning. Additionally, we simulated the response of simple macrocycles with $\mathrm{O}, \mathrm{N}$, and $\mathrm{S}$ within the same ring system. Our observations demonstrated that these simple macrocyclic structures have the capacity to undergo high distortions albeit low energy changes.

By using $18 \mathrm{C} 6$ as the starting structure, other simple macrocycles were simulated to elucidate the possible factors and interactions that take place in solution. In the presence of metal species such as $\mathrm{Ba}^{2+}$ or $\mathrm{Pb}^{2+}$, there is considerable displacement of these ions when exposed to common solvents such as methanol, acetonitrile, and water. Because of this, these metal ions are more prone to forming "sandwich" or 1:2 M-L structures. The results also indicate 18C6 has selectivity of $\mathrm{Tl}>\mathrm{Ba}>\mathrm{Pb}$ which correspond to DFT findings. Finally, the findings show that crown ethers can complex with Sb but to an unfavorable distortion of the base structure of the ligand.

Utilizing macrocycles for simplistic determinations in ESI-MS experiments is beneficial as they can preserve the natural abundances of metallic species. However, the selectivity of macrocycles can be altered via heteroatom substitution within the ring structure. These distortions relate to the strength of non-bonded interactions between the host and guest, which then teaches about the overall host-guest interaction energy. It was found through DFT calculations that sulfur atoms were effective in binding $\mathrm{Sb}^{3+}$ and $\mathrm{Tl}^{+}$cations due to the chemical softness of both the ligand and guests. Additionally, using solvation strategies and calculating molecular descriptors (HOMO 
and LUMO), further insight into the factors governing the formation of "sandwich" structures and selectivity can be achieved. Hence, this work demonstrates the value of DFT to probe for valuable information and structures obtained from ESI-MS/MS experiments.

\subsection{Future Work}

The use of gunshot residue as a means of informative and important evidence has suffered due to problematic formulation shifts, lengthy analysis times, and difficulties of incorporating all IGSR and OGSR information from a collected sample. This collection of work, as well as the efforts made by other researchers, have started adapting and investigating the underlying complexities involved in a deflagration event and following post-shooting activities. However, there is still more room for further considerations and investigations in GSR. One possible expansion is the need of larger datasets that incorporate various geographical locations. By comparing and investigating different areas for the commonality of GSR in the environment, participating laboratories can provide more objective information in the form of likelihood ratios.

Another avenue that would assist the GSR and forensics communities is to ultimately assess the use of the proposed method and interpretation approach with casework samples. This can be achieved through interlaboratory/pilot collaborations with crime labs. This is important because the current practices must adapt to the shift of "environmentally" friendly formulations and increase in backlogs. Hence, more rapid instrumentation and more objective statistical methodologies should be implemented to alleviate the burden placed on these labs.

Finally, there is one major nuisance and question when regarding other individuals associated with or near (bystanders) firearm-related incidents. These bystander studies could have high GSR counts and concentrations on their hands which may either lead into false positive rates or worse, wrongful convictions. Therefore, researchers should consider expanding current 
investigations to transfer rates of GSR particulates, including OGSR compounds. Once possible way to study these instances is by having individuals encountering various surfaces and objects after a discharge event has occurred. Another avenue is studying the dispersion and expansion of these analytes using high-resolution imaging strategies. Thus, if these questions are properly addressed and answered, law enforcement would have a greater understanding of the events occurring while also evaluating several hypotheses.

Shifting from trace evidence, the strategy of host-guest chemistry can be further expanded into other areas in forensic science as well as broader analytical applications. For the forensics community, the strategies outlined in previous chapters can be applied to other materials, such as drugs-of-abuse; more specifically fentanyl-related compounds (FRCs). The concern of FRCs is their ability to mimic opiate responses within the nervous system [1]. These responses are achieved by slightly altering the core structure's functional groups " $R$-groups" (outlined in Figure 7.1) and expands into other novel psychoactive substance drug classes, like cathinones and cannabinoids. Therefore, slight modifications can result in hundreds of unique isomers unclassified by law enforcement.

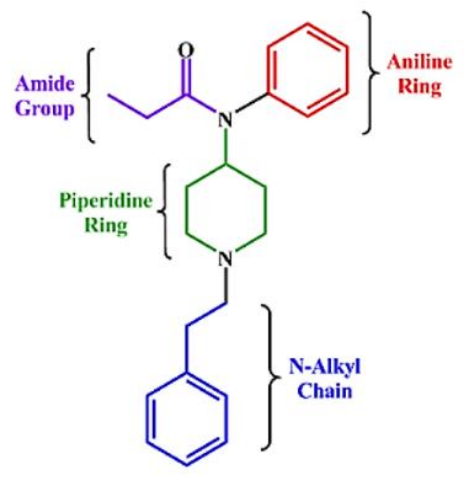

Figure 7.1: Typical locations for group substitution FRCs.

While instrumentation like gas chromatography-mass spectrometry (GC-MS) and liquid chromatography-tandem mass spectrometry (LC-MS/MS) can detect many of these modifications, 
isomeric species produce similar mass spectra and identical retention/elution times. Conversely, forensic drug laboratories possess IR or Raman capabilities but only deploy them in specific cases such as explosive detection [2]. These spectral and chromatographic similarities decrease the confidence in classification and require additional exploration using statistical methodologies. Furthermore, adulterants found in complex mixtures can further complicate data analysis by suppressing analyte signals.

To circumvent some of these challenges, selective host-guest interactions can produce unique traits to circumvent signal suppression easily and unlock definitive isomeric identification in both screening and confirmatory methods as it leverages a large organic macromolecule (host) to interact, or bind, with an analyte. A guest, in this case, an FRC, may orient and position itself in the most stable conformations within the host's inner cavity as seen in Figure 7.2.

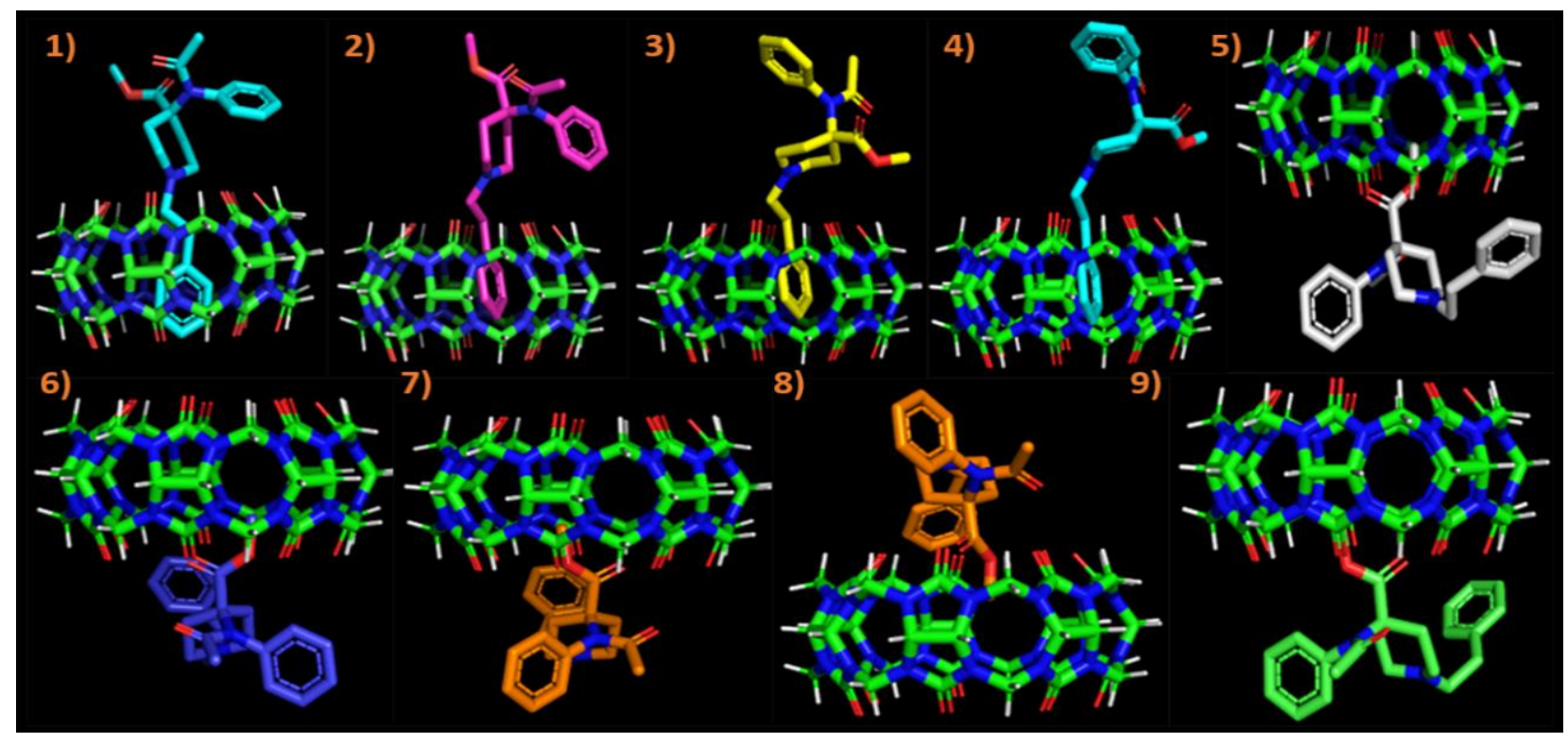

Figure 7.2: Preliminary evaluation and interaction of carfentanil with cucurbit[7]uril.

Depending on the host's chemical composition and environment, various interactions can occur, including ion-dipole, non-covalent, electrostatic, and van der Waals forces. In turn, this causes the host to restructure to the lowest energy conformation and yield unique spectral responses. This 
approach allows for the FRC's chemical composition to be maintained while simultaneously allowing shifts in $\mathrm{m} / \mathrm{z}$ values (for MS) and spectral signals (for Raman and IR). Depending on the interactions that occur, these shifts can be sufficient to differentiate previously indistinguishable isomers. Fortunately, DFT calculations can provide insights to the most favorable energy conformation as well as simulated IR and Raman spectra with high accuracy.

In short, host-guest chemistry can provide additional strategies for not only chemists but for forensic scientists. The most commonly studied host molecules are crown ethers, first introduced in the forensics community to detect gunshot residue whereas more complex macrocycles include cucurbit[ $n]$ urils and cyclodextrins and have been efficient for drug delivery systems [3,4]. Strategies such as quantum mechanics (DFT) can filter out inefficient hosts and reduce physical costs and resources to decrease the time searching for optimum hosts. Therefore, by leveraging DFT calculations, host-guest chemistry, and machine learning and multivariate methods, can assist laboratories to potentially unlock confident isomer identification. Furthermore, the knowledge gained through these works allows for a base for future scientists to expand and improve upon. 


\subsection{References for Chapter 7}

[1] C.A.F. De Oliveira Penido, M.T.T. Pacheco, I.K. Lednev, L. Silveira, Raman spectroscopy in forensic analysis: Identification of cocaine and other illegal drugs of abuse, J. Raman Spectrosc. 47 (2016) 28-38. doi:10.1002/jrs.4864.

[2] E. Sisco, J. Verkouteren, J. Staymates, J. Lawrence, Rapid detection of fentanyl, fentanyl analogues, and opioids for on-site or laboratory-based drug seizure screening using thermal desorption DART-MS and ion mobility spectrometry, Forensic Chem. 4 (2017) 108-115. doi:10.1016/j.forc.2017.04.001.

[3] S.R. Ahmed, R. Chand, S. Kumar, N. Mittal, S. Srinivasan, A.R. Rajabzadeh, Recent biosensing advances in the rapid detection of illicit drugs, TrAC - Trends Anal. Chem. 131 (2020). doi:10.1016/j.trac.2020.116006.

[4] A.I. Lazar, F. Biedermann, K.R. Mustafina, K.I. Assaf, A. Hennig, W.M. Nau, Nanomolar Binding of Steroids to Cucurbit[n]urils: Selectivity and Applications, J. Am. Chem. Soc. 138 (2016) 13022-13029. doi:10.1021/jacs.6b07655. 


\section{Appendices}

Appendix I-Chapter 4: Detection of Organic and Inorganic Gunshot Residues from Hands using Complexing Agents and LC-MS/MS - Supplemental Information

Table S4.1: Detailed description of the mobile phase composition and times for the LC.

\begin{tabular}{c|c|c}
\hline Time (min) & Water w/ 0.1\% FA (A\%) & Acetonitrile w/ 0.1\% FA (B\%) \\
\hline 0.00 & 80 & 20 \\
\hline 1.30 & 55 & 45 \\
\hline 2.00 & 50 & 50 \\
\hline 2.40 & 45 & 55 \\
\hline 3.30 & 35 & 65 \\
\hline 4.20 & 32 & 68 \\
\hline 4.50 & 30 & 70 \\
\hline 5.30 & 28 & 72 \\
\hline 6.50 & 25 & 75 \\
\hline 8.00 & 5 & 95 \\
\hline 9.00 & 90 & 10 \\
\hline
\end{tabular}

Table S4.2: Detailed description of the source parameters for the MS/MS.

\begin{tabular}{c|c|c}
\hline \multicolumn{2}{|c|}{ Source Parameters } \\
\hline Gas Temperature & \multicolumn{2}{|c}{$300^{\circ} \mathrm{C}$} \\
\hline Gas Flow & \multicolumn{2}{|c}{$101 / \mathrm{min}$} \\
\hline Nebulizer & \multicolumn{2}{|c}{$20 \mathrm{psi}$} \\
\hline $\begin{array}{c}\text { Sheath Gas } \\
\text { Temperature }\end{array}$ & \multicolumn{2}{|c}{$250^{\circ} \mathrm{C}$} \\
\hline Sheath Gas Flow & \multicolumn{2}{|c}{$71 / \mathrm{min}$} \\
\hline \multirow{2}{*}{ Capillary } & Positive & Negative \\
\cline { 2 - 3 } & $4500 \mathrm{~V}$ & $2500 \mathrm{~V}$ \\
\hline \multirow{2}{*}{ Nozzle } & Positive & Negative \\
\cline { 2 - 3 } & $2000 \mathrm{~V}$ & $2000 \mathrm{~V}$ \\
\hline
\end{tabular}


Figure S4.1: Comparison of responses of barium and lead within one solution using 18C6 complexing agent. Additionally, the measured response and proposed theoretical structure of thallium and 18C6.
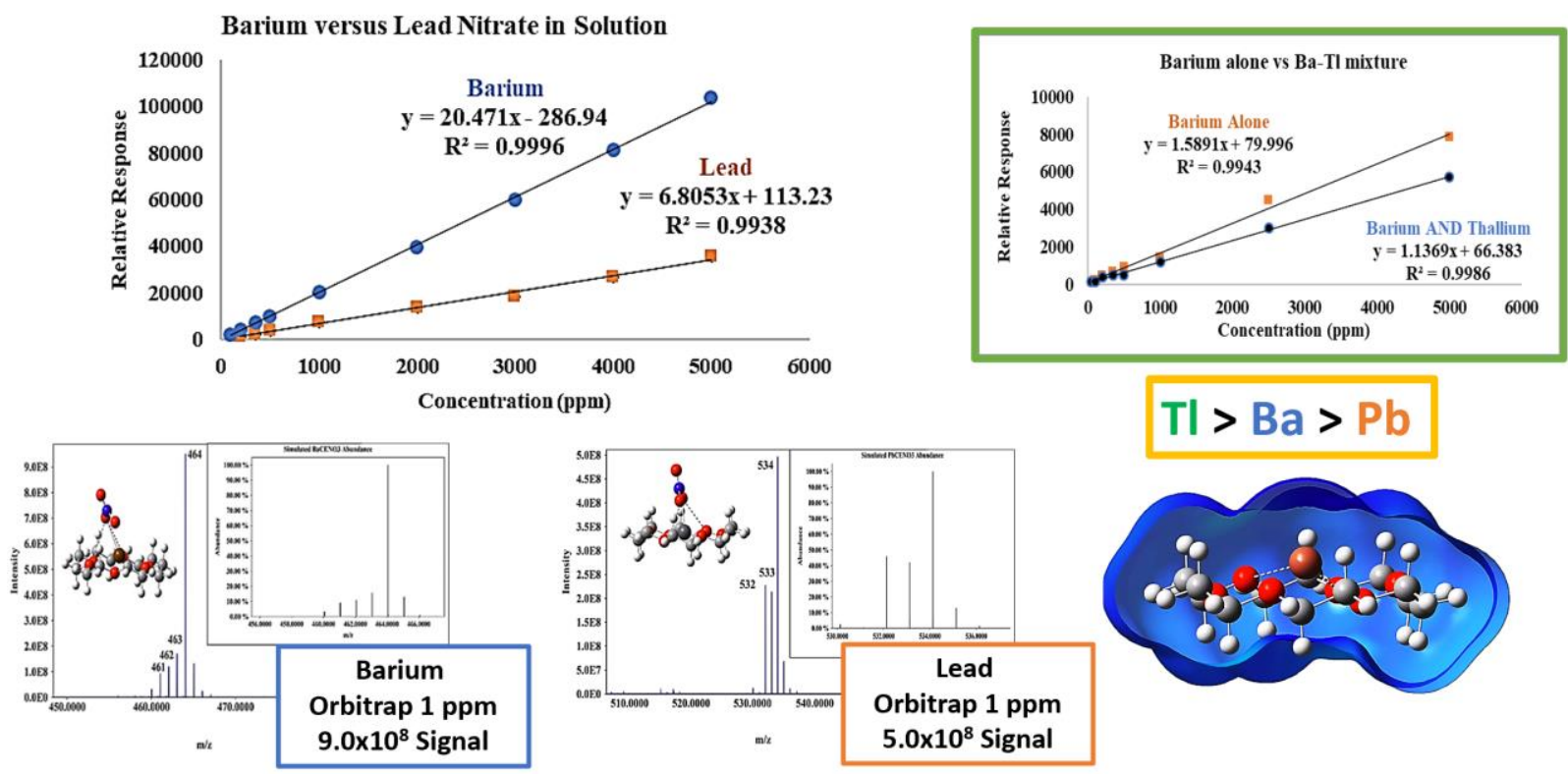

\section{$\mathrm{Tl}>\mathrm{Ba}>\mathrm{Pb}$}

Figure S4.2: Example of intra- and interday variability of methyl centralite along with the residual plot of the working range. These figures demonstrate the linearity and response of this OGSR compound and the randomness and homoscedasticity of the points across all concentration levels.
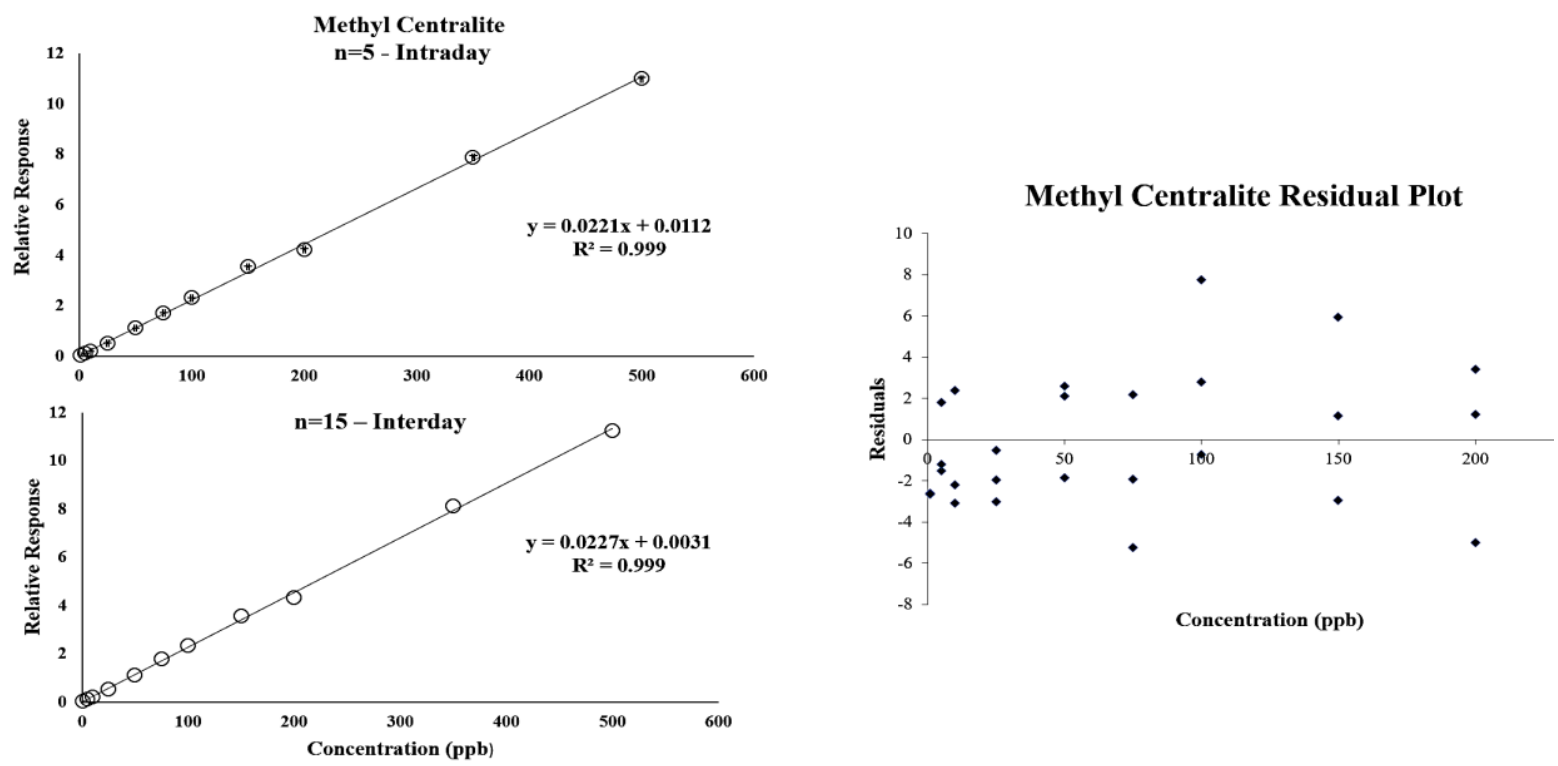
Table S4.3: Detailed definitions of the Figures of Merit outlined by the Eurachem guidelines. Included are the equations associated with the corresponding validation parameter.

\begin{tabular}{|c|c|c|}
\hline $\begin{array}{l}\text { Figure of } \\
\text { Merit }\end{array}$ & Definition & Equation \\
\hline Selectivity & $\begin{array}{l}\text { The ability of a method to distinguish analytes without interferences. Techniques such as liquid } \\
\text { chromatography and mass spectrometric data are helpful to distinguish both elution and } \\
\text { fragmentation patterns. Selectivity testing measures standards against other independent methods } \\
\text { and test the samples against possible interferences. }\end{array}$ & $\mathbf{N} / \mathbf{A}$ \\
\hline $\begin{array}{l}\text { Limit of } \\
\text { Detection }\end{array}$ & $\begin{array}{l}\text { The lowest concentration of an analyte detectable at a specified confidence level. Ten blank } \\
\text { measurements typically determine it without analytes or } 10 \text { replicates of low concentrations. } \\
\text { Equation } 1 \text { refers to the lowest value at which an accurate detection of an analyte is present or } \\
\text { absent qualitatively. }\end{array}$ & $\mathbf{L O D}=\mathbf{3} * \frac{\mathbf{S}}{\mathbf{N}}$ \\
\hline $\begin{array}{l}\text { Limit of } \\
\text { Quantitation }\end{array}$ & $\begin{array}{l}\text { The lowest concentration of an analyte performance is deemed acceptable for a specified } \\
\text { application. Ten blank measurements typically determine it without analytes or } 10 \text { replicates of low } \\
\text { concentrations. The lowest value at which the analyte concentration can accurately quantitatively. }\end{array}$ & LOD $=10 * \frac{s}{N}$ \\
\hline Sensitivity & A change in instrument response, which corresponds to a change in the measured quantity. & $\mathbf{N} / \mathbf{A}$ \\
\hline $\begin{array}{l}\text { Working } \\
\text { Range }\end{array}$ & $\begin{array}{l}\text { Interval over which the method provides results with an acceptable uncertainty. The lower end of a } \\
\text { working range is restricted to the LOQ, while significant anomalies in concentrations affecting the } \\
\text { analytical sensitivity define the upper end. In this case, residual plots allow for more refined } \\
\text { explanations and limitations of calibration curves as they must fall below } \pm 20 \% \text { of expected } \\
\text { concentrations. }\end{array}$ & $\mathbf{N} / \mathbf{A}$ \\
\hline Bias & $\begin{array}{l}\text { Comparing the mean of the results }(\overline{\mathrm{x}}) \text { from the candidate method with a suitable reference value } \\
\left(x_{r e f}\right) \text { and calculated. Bias typically describes the percent recovery of a particular extraction } \\
\text { procedure, such as collection substrates. }\end{array}$ & $\begin{array}{l}\boldsymbol{R}(\%) \\
=\frac{\overline{\boldsymbol{x}}}{\boldsymbol{x}_{\text {ref }}} * \mathbf{1 0 0}\end{array}$ \\
\hline
\end{tabular}


Appendix II - Chapter 5: Evaluation of Organic and Inorganic Gunshot Residues in Various Populations using LC-MS/MS - Supplemental Information

Table S5.1: Descriptive statistics (mean and standard deviations) of concentrations of OGSR $(\mu \mathrm{g} / \mathrm{L})$ and IGSR $(\mu \mathrm{g} / \mathrm{mL})$ compounds on the various subpopulations. Sets are comprised of various numbers of hand samples collected (either 2 (left- and right hands) or 4 (left (palm and back) and right (palm and back) hands).

\begin{tabular}{|c|c|c|c|c|c|}
\hline Analyte & $\begin{array}{c}\text { Shooter baseline } \\
(n=246)\end{array}$ & $\begin{array}{l}\text { Post-shooting } \\
\text { activity }(n=78)\end{array}$ & $\begin{array}{l}\text { Bystanders } \\
(\mathrm{n}=151)\end{array}$ & $\begin{array}{c}\text { Low-risk } \\
\text { background }(\mathrm{n}=157)\end{array}$ & $\begin{array}{c}\text { High-risk } \\
\text { background }(\mathrm{n}=97)\end{array}$ \\
\hline AK 2 & $17 \pm 43$ & $7.3 \pm 29$ & ND & ND & ND \\
\hline MC & ND & ND & ND & ND & ND \\
\hline EC & $38 \pm 98$ & $20 \pm 50$ & $0.4 \pm 1.6$ & $1.0 \pm 1.0$ & $1.0 \pm 1.0$ \\
\hline DPA & $16 \pm 45$ & $27 \pm 56$ & ND & ND & ND \\
\hline N-NDPA & $2.0 \pm 13$ & $21 \pm 58$ & ND & ND & ND \\
\hline 4-NDPA & $3.0 \pm 7.0$ & $3.0 \pm 8.8$ & ND & ND & ND \\
\hline 2-NDPA & $38 \pm 229$ & $2.2 \pm 7.0$ & ND & ND & ND \\
\hline $\mathbf{B a}$ & $2728 \pm 1115$ & $801 \pm 222$ & $240 \pm 101$ & ND & $454 \pm 276$ \\
\hline $\mathbf{P b}$ & $1354 \pm 597$ & $479 \pm 472$ & ND & ND & $88 \pm 120$ \\
\hline
\end{tabular}

Table S5.2: Average correct classification rates for the neural network using Min-Max scaling. Here, 10 replicate random iterations were tested and averaged using the 60:20:20 ratio of training, validation, and testing. Data represents the percent accuracy and respective coefficient of variation across the iterations.

\begin{tabular}{|c|c|c|c|}
\hline \begin{tabular}{|l|} 
Group Set \\
\end{tabular} & $60 \%$ Training & $20 \%$ Validation & $20 \%$ Testing \\
\hline Shooter & $99.6 \pm 0.5$ & $99.1 \pm 0.6$ & $99 \pm 1$ \\
\hline Activity & $93 \pm 13$ & $93 \pm 5$ & $93 \pm 5$ \\
\hline Low-Risk & $98.7 \pm 0.3$ & $98.8 \pm 0.6$ & $99 \pm 1$ \\
\hline High-Risk & $91 \pm 3$ & $91 \pm 2$ & $90 \pm 3$ \\
\hline Bystander & $97 \pm 0.9$ & $95 \pm 1$ & $96 \pm 2$ \\
\hline
\end{tabular}

Table S5.3: The JMP Pro ${ }^{\circledR}$ software divides the input data into three categories internal to the network creation and optimization process: training, testing, and internal validation. The user sets these percentages, 60\%:20\%:20\% respectively. This means if a data file consisted of 100 spectra, 60 would be used in the iterative adjusting of weights (training), 20 would be used to test the resulting network solutions and back propagate errors, and 20 would be used to measure the network performance for comparison to user-specified acceptance criteria. This practice is used to ensure that a network solution (based on training and test data) can successfully generalize the solution to spectra not involved in the training/testing process.

\begin{tabular}{|l|l|}
\hline Setting & Values \\
\hline \% Training & 60 \\
\hline \% Validation & 20 \\
\hline \% Testing & 20 \\
\hline Hidden Layer Structure & Sigmoid \\
\hline Nodes & 3 \\
\hline
\end{tabular}




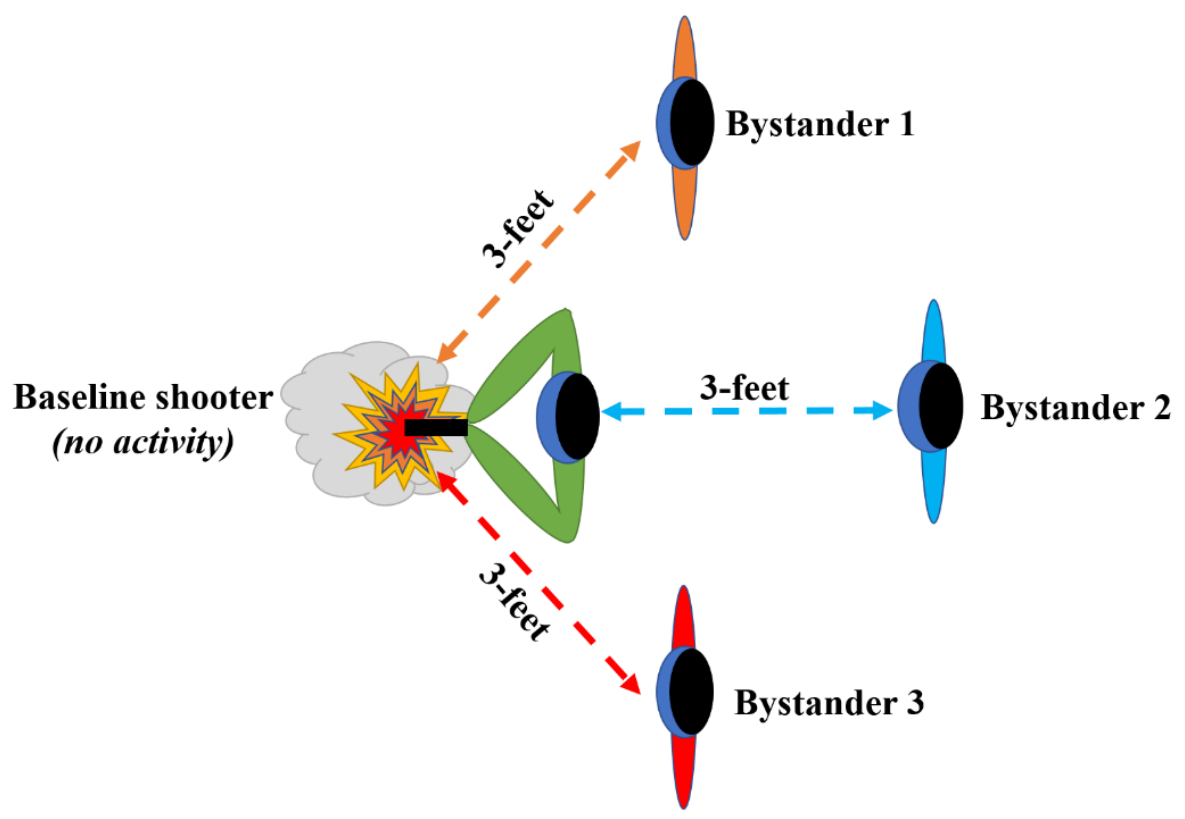

Figure S5.1: Graphical representation of the bystander shooting set. 
Appendix III - Chapter 6: Investigations into host-guest interactions with metal ions using DFT for applications using mass spectrometry - Supplemental Information

Figure S6.1: Linear responses for $\mathrm{BaNO}_{3}$ and $\mathrm{PbNO}_{3}(\mathrm{LEFT})$ and $\mathrm{BaNO}_{3}$ and $\mathrm{Tl}$ (RIGHT) in the presence of 18C6 analyzed by LC-MS/MS.
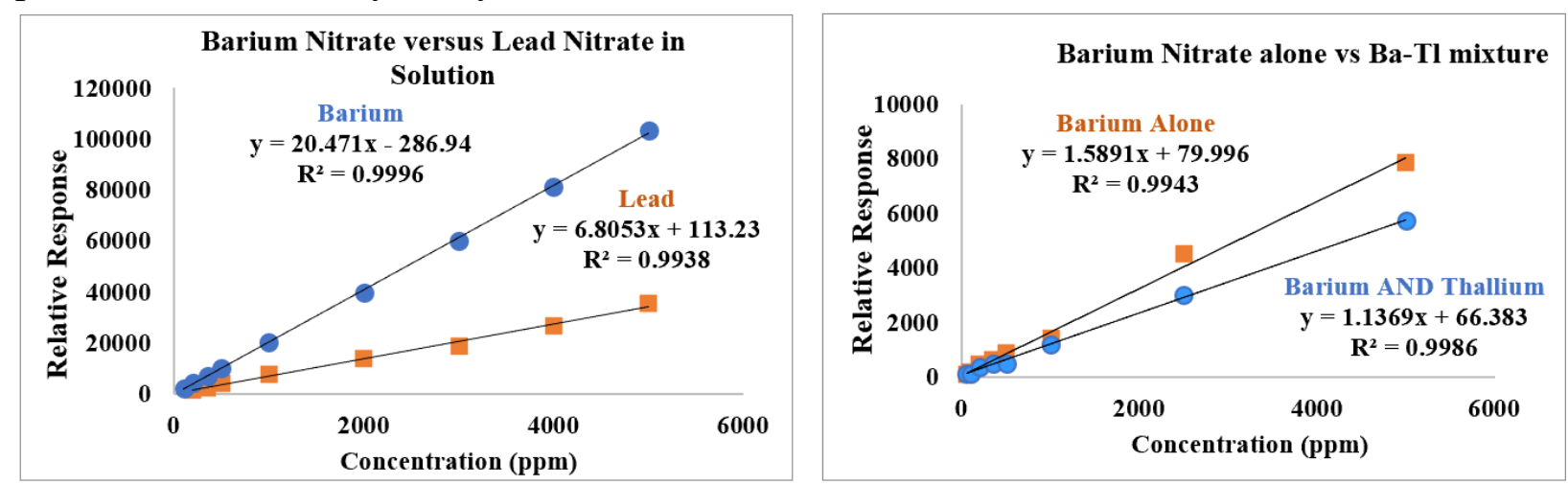

Table S6.1: Molecular orbital energies and global reactivity descriptors of ligands. All data are based on different phases of DFT calculations at B3LYP/6-311++G (2d, p) level. Here some of the heteroatom substituted macrocycles properties such as LUMO and HOMO energies, difference of the orbitals $\Delta \mathrm{E}=\mathrm{E}_{\mathrm{Lumo}}-\mathrm{E}_{\text {HOmo, }}$, the chemical hardness $(\eta)$, and global softness $(S)$ of the ligand.

\begin{tabular}{|c|c|c|c|c|c|c|}
\hline Ligand & Phase & ELuMo $_{\text {Lu }}$ & Еномо & $\Delta \mathbf{E}$ & $\eta$ & $\mathbf{S}$ \\
\hline \multirow{4}{*}{$\mathrm{C}_{12} \mathrm{H}_{24} \mathrm{O}_{6}$} & Gas & 0.307 & -0.281 & 0.588 & 0.294 & 3.401 \\
\hline & $\mathrm{ACN}$ & 0.309 & -0.285 & 0.594 & 0.297 & 3.367 \\
\hline & $\mathrm{MeOH}$ & 0.296 & -0.299 & 0.595 & 0.2975 & 3.361 \\
\hline & $\mathrm{H}_{2} \mathrm{O}$ & 0.294 & -0.300 & 0.594 & 0.297 & 3.367 \\
\hline \multirow{4}{*}{$\mathrm{C}_{12} \mathrm{H}_{25} \mathrm{NO}_{5}$} & Gas & 0.313 & -0.267 & 0.580 & 0.29 & 3.448 \\
\hline & $\mathrm{ACN}$ & 0.007 & -0.222 & 0.222 & 0.111 & 3.409 \\
\hline & $\mathrm{MeOH}$ & 0.297 & -0.293 & 0.590 & 0.295 & 3.389 \\
\hline & $\mathrm{H}_{2} \mathrm{O}$ & 0.294 & -0.298 & 0.592 & 0.296 & 3.378 \\
\hline \multirow{4}{*}{$\mathrm{C}_{12} \mathrm{H}_{26} \mathrm{~N}_{2} \mathrm{O}_{4}$} & Gas & -0.007 & -0.204 & 0.197 & 0.0985 & 3.452 \\
\hline & $\mathrm{ACN}$ & 0.004 & -0.213 & 0.213 & 0.1065 & 3.389 \\
\hline & $\mathrm{MeOH}$ & 0.297 & -0.287 & 0.584 & 0.292 & 3.424 \\
\hline & $\mathrm{H}_{2} \mathrm{O}$ & -0.00037 & -0.22 & 0.220 & 0.109815 & 3.106 \\
\hline \multirow{4}{*}{$\mathrm{C}_{12} \mathrm{H}_{27} \mathrm{~N}_{3} \mathrm{O}_{3}$} & Gas & -0.008 & -0.207 & 0.199 & 0.0995 & 3.050 \\
\hline & $\mathrm{ACN}$ & 0.008 & -0.214 & 0.214 & 0.107 & 3.345 \\
\hline & $\mathrm{MeOH}$ & 0.004 & -0.216 & 0.216 & 0.108 & 3.259 \\
\hline & $\mathrm{H}_{2} \mathrm{O}$ & -0.00038 & -0.219 & 0.219 & 0.10931 & 3.148 \\
\hline \multirow{4}{*}{$\mathrm{C}_{12} \mathrm{H}_{28} \mathrm{~N}_{4} \mathrm{O}_{2}$} & Gas & -0.009 & -0.206 & 0.197 & 0.0985 & 3.152 \\
\hline & $\mathrm{ACN}$ & -0.007 & -0.214 & 0.214 & 0.107 & 3.345 \\
\hline & $\mathrm{MeOH}$ & -0.006 & -0.215 & 0.215 & 0.1075 & 3.302 \\
\hline & $\mathrm{H}_{2} \mathrm{O}$ & -0.008 & -0.218 & 0.218 & 0.109 & 3.174 \\
\hline \multirow{4}{*}{$\mathrm{C}_{12} \mathrm{H}_{29} \mathrm{~N}_{5} \mathrm{O}$} & Gas & 0.313 & -0.265 & 0.578 & 0.289 & 3.460 \\
\hline & $\mathrm{ACN}$ & 0.315 & -0.282 & 0.597 & 0.2985 & 3.350 \\
\hline & $\mathrm{MeOH}$ & 0.304 & -0.287 & 0.591 & 0.2955 & 3.384 \\
\hline & $\mathrm{H}_{2} \mathrm{O}$ & 0.301 & -0.294 & 0.595 & 0.2975 & 3.361 \\
\hline \multirow{2}{*}{$\mathrm{C}_{12} \mathrm{H}_{30} \mathrm{~N}_{6}$} & Gas & 0.372 & -0.267 & 0.639 & 0.3195 & 3.129 \\
\hline & $\mathrm{ACN}$ & 0.371 & -0.286 & 0.657 & 0.3285 & 3.044 \\
\hline
\end{tabular}




\begin{tabular}{|c|c|c|c|c|c|c|}
\hline & $\begin{array}{c}\mathrm{MeOH} \\
\mathrm{H}_{2} \mathrm{O}\end{array}$ & $\begin{array}{c}0.37 \\
0.367 \\
\end{array}$ & $\begin{array}{l}-0.288 \\
-0.294 \\
\end{array}$ & $\begin{array}{l}0.658 \\
0.661 \\
\end{array}$ & $\begin{array}{c}0.329 \\
0.3305 \\
\end{array}$ & $\begin{array}{l}3.039 \\
3.025 \\
\end{array}$ \\
\hline \multirow{4}{*}{$\mathrm{C}_{12} \mathrm{H}_{24} \mathrm{O}_{5} \mathrm{~S}$} & Gas & -0.0067 & -0.204 & 0.197 & 0.09865 & 5.136 \\
\hline & $\mathrm{ACN}$ & 0.164 & -0.223 & 0.387 & 0.0935 & 5.167 \\
\hline & $\mathrm{MeOH}$ & 0.161 & -0.226 & 0.387 & 0.0935 & 5.167 \\
\hline & $\mathrm{H}_{2} \mathrm{O}$ & 0.160 & -0.227 & 0.387 & 0.0935 & 5.167 \\
\hline \multirow{4}{*}{$\mathrm{C}_{12} \mathrm{H}_{24} \mathrm{O}_{4} \mathrm{~S}_{2}$} & Gas & 0.166 & -0.214 & 0.380 & 0.19 & 5.263 \\
\hline & $\mathrm{ACN}$ & 0.161 & -0.224 & 0.385 & 0.1925 & 5.194 \\
\hline & $\mathrm{MeOH}$ & 0.16 & -0.225 & 0.385 & 0.1925 & 5.194 \\
\hline & $\mathrm{H}_{2} \mathrm{O}$ & 0.159 & -0.226 & 0.385 & 0.1925 & 5.194 \\
\hline \multirow{4}{*}{$\mathrm{C}_{12} \mathrm{H}_{24} \mathrm{O}_{3} \mathrm{~S}_{3}$} & Gas & 0.156 & -0.211 & 0.367 & 0.1835 & 5.449 \\
\hline & $\mathrm{ACN}$ & 0.155 & -0.223 & 0.378 & 0.189 & 5.291 \\
\hline & $\mathrm{MeOH}$ & 0.158 & -0.225 & 0.383 & 0.1915 & 5.221 \\
\hline & $\mathrm{H}_{2} \mathrm{O}$ & 0.158 & -0.226 & 0.384 & 0.192 & 5.208 \\
\hline \multirow{4}{*}{$\mathrm{C}_{12} \mathrm{H}_{24} \mathrm{O}_{2} \mathrm{~S}_{4}$} & Gas & 0.155 & -0.221 & 0.376 & 0.188 & 5.319 \\
\hline & $\mathrm{ACN}$ & 0.157 & -0.224 & 0.381 & 0.1905 & 5.249 \\
\hline & $\mathrm{MeOH}$ & 0.158 & -0.225 & 0.383 & 0.1915 & 5.221 \\
\hline & $\mathrm{H}_{2} \mathrm{O}$ & 0.158 & -0.226 & 0.384 & 0.192 & 5.208 \\
\hline \multirow{4}{*}{$\mathrm{C}_{12} \mathrm{H}_{24} \mathrm{OS}_{5}$} & Gas & 0.151 & -0.216 & 0.367 & 0.1835 & 5.449 \\
\hline & $\mathrm{ACN}$ & 0.156 & -0.224 & 0.380 & 0.19 & 5.263 \\
\hline & $\mathrm{MeOH}$ & 0.158 & -0.225 & 0.383 & 0.1915 & 5.221 \\
\hline & $\mathrm{H}_{2} \mathrm{O}$ & 0.154 & -0.227 & 0.381 & 0.1905 & 5.249 \\
\hline \multirow{4}{*}{$\mathrm{C}_{12} \mathrm{H}_{24} \mathrm{~S}_{6}$} & Gas & -0.0981 & -0.358 & 0.260 & 0.12995 & 7.695 \\
\hline & $\mathrm{ACN}$ & 0.159 & -0.225 & 0.384 & 0.192 & 5.208 \\
\hline & $\mathrm{MeOH}$ & 0.160 & -0.225 & 0.385 & 0.1925 & 5.194 \\
\hline & $\mathrm{H}_{2} \mathrm{O}$ & 0.160 & -0.226 & 0.386 & 0.193 & 5.181 \\
\hline
\end{tabular}


Table S6.2: Description of the interaction energy contributing from the 18C6 to the metal guest (Ba). $\mathrm{E}(2)$ is the interaction energy, $\varepsilon_{i}$ and $\varepsilon_{j}$ are the diagonal elements (orbital energies), $F(i, j)$ is the off-diagonal element, LP and LP* are the lone pair contributors (bonding and antibond), and $\mathrm{RY}$ is the Ryberg factor of electrons moving between energy levels.

\begin{tabular}{|c|c|c|c|c|}
\hline 18C6 Factor & Ba Factor & $\mathrm{E}(2) \mathrm{kcal} / \mathrm{mol}$ & $E(\mathbf{j})-\mathbf{E}(\mathbf{i})$ & $F(\mathbf{i}, \mathbf{j})$ \\
\hline LP(1)O1 & LP*(1)Ba & 2.14 & 0.76 & 0.036 \\
\hline LP(1)O1 & RY*(4)Ba & 0.22 & 6.47 & 0.034 \\
\hline LP(1)O1 & $\mathbf{R Y} *(\mathbf{6}) \mathbf{B a}$ & 0.16 & 2.89 & 0.019 \\
\hline LP(2)O1 & $\mathbf{R Y} *(3) \mathbf{B a}$ & 0.06 & 0.84 & 0.006 \\
\hline LP(1)O8 & $\mathbf{L P} *(\mathbf{1}) \mathbf{B a}$ & 2.25 & 0.78 & 0.038 \\
\hline LP(1)O8 & RY*(4)Ba & 0.38 & 6.48 & 0.045 \\
\hline LP(1)O8 & $\mathbf{R Y} *(\mathbf{6}) \mathbf{B a}$ & 0.24 & 2.9 & 0.024 \\
\hline LP(1)O8 & $\mathbf{R Y} *(7) \mathbf{B a}$ & 0.06 & 1.09 & 0.007 \\
\hline LP(2)O8 & $\mathbf{R Y} *(3) \mathbf{B a}$ & 0.07 & 0.84 & 0.007 \\
\hline LP(1)015 & $\mathbf{L P} *(\mathbf{1}) \mathbf{B a}$ & 2.11 & 0.77 & 0.036 \\
\hline LP(1)O15 & RY*(4)Ba & 0.08 & 0.92 & 0.008 \\
\hline LP(1)O15 & $\mathbf{R Y} *(6) \mathbf{B a}$ & 0.3 & 6.48 & 0.04 \\
\hline LP(1)O15 & $\mathbf{R Y} *(7) \mathbf{B a}$ & 0.21 & 2.89 & 0.022 \\
\hline LP(2)015 & $\mathbf{R Y} *(\mathbf{1}) \mathbf{B a}$ & 0.1 & 0.64 & 0.007 \\
\hline $\mathbf{L P}(\mathbf{1}) \mathbf{O 2 2}$ & $\mathbf{L P} *(\mathbf{1}) \mathbf{B a}$ & 2.2 & 0.77 & 0.037 \\
\hline LP(1)O22 & RY*(4)Ba & 0.31 & 6.48 & 0.04 \\
\hline LP(1)O22 & $\mathbf{R Y} *(6) \mathbf{B a}$ & 0.19 & 2.89 & 0.021 \\
\hline LP(1)O22 & $\mathbf{R Y} *(7) \mathbf{B a}$ & 0.09 & 1.08 & 0.009 \\
\hline $\mathbf{L P}(2) 022$ & $\mathbf{R Y} *(\mathbf{1}) \mathbf{B a}$ & 0.13 & 0.64 & 0.008 \\
\hline LP(1)O29 & $\mathbf{L P} *(\mathbf{1}) \mathbf{B a}$ & 2.09 & 0.76 & 0.036 \\
\hline LP(1)O29 & $\mathbf{R Y} *(4) \mathbf{B a}$ & 0.21 & 6.47 & 0.033 \\
\hline LP(1)O29 & $\mathbf{R Y} *(\mathbf{6}) \mathbf{B a}$ & 0.14 & 2.89 & 0.018 \\
\hline LP(1)O36 & $L P *(1) B a$ & 2.35 & 0.77 & 0.038 \\
\hline LP(1)O36 & $\mathbf{R Y} *(\mathbf{1}) \mathbf{B a}$ & 0.08 & 0.92 & 0.008 \\
\hline LP(1)O36 & $\mathbf{R Y} *(4) \mathbf{B a}$ & 0.38 & 6.48 & 0.045 \\
\hline LP(1)O36 & $\mathbf{R Y} *(\mathbf{6}) \mathbf{B a}$ & 0.27 & 2.89 & 0.025 \\
\hline LP(1)O36 & $\mathbf{R Y} *(7) \mathbf{B a}$ & 0.06 & 1.08 & 0.007 \\
\hline LP(2)O36 & RY*(1)Ba & 0.11 & 0.64 & 0.008 \\
\hline
\end{tabular}


Appendix IV-Professional CV

William Jansen Feeney

ORCID: 0000-0001-7218-3449

EDUCATION

PhD, Chemistry

2016-2021

GPA- 3.884

West Virginia University

Morgantown, WV
BS, Chemistry

2012-2016

GPA- 3.33

West Virginia University

Morgantown, WV
BS, Forensic and Investigative Sciences

2012-2016

GPA- 3.33

West Virginia University

Morgantown, WV

\section{PROFESSIONAL WORK EXPERIENCE}

Graduate Research Assistant - Chemistry | June 2016 - Present

Dissertation Title: Exploration and Evaluation of Analytical Methodologies Utilizing Host-Guest Interactions including Macromolecules and Binding Agents with Multiple Metal Species in Firearm Discharge Residue

- Support and mentor undergraduates through the experimental design process

- Troubleshoot and maintain instrumentation on multiple vendor platforms, communicating with manufacturers and technical support when required

- Determine interaction pathways of guest analytes with host molecules via binding coefficients, and intensities

- Monitored interactions pathways and binding using quantum chemical models (density functional theory (DFT)

- Identify characteristic fragmentation patterns for species of interest and determine statistical viability for monitoring for proof-of-concept study

- Develop and validate HPLC and GCMS methods for explosive materials (firearm discharge residue)

Graduate Teaching Assistant - Chemistry | January 2017 to May 2018

West Virginia University

- Controlled and accounted for controlled substances before, during, and after laboratory exercises

- Developed experimental methods for GCMS and FTIR laboratory exercises and instructed students on basic software functions and data collation

- Demonstrated proper use of laboratory equipment and ensured students adhered to safety guidelines while performing experiments

- Prepared materials such as quizzes, supplementary handouts, and procedures

- Evaluated students and provided constructive feedback on assignments and laboratory notebooks 
Undergraduate Research Assistant - Chemistry | December 2015 to May 2016 West Virginia University

- Developed GCMS methods for the analysis of organic molecules related to explosive materials. Identified and characterized potential organic analytes of interest related to firearm discharge residue

Undergraduate Research Assistant - Chemistry | July 2014 to December 2014

West Virginia University

- Monitored the effects of endocrine disrupting chemicals on zebrafish utilizing capillary electrophoresis

- Developed extraction method for endocrine disrupting chemicals in water

Conducted regular instrument maintenance and performed data collection on capillary electrophoresis

\section{ANALYTICAL, THEORETICAL, AND STATISTICAL TECHNIQUES}

- Analytical Instrumentation

○ Liquid chromatography (LC) (separation)

- Gas chromatography (GC) (separation)

- Ion Mobility Spectrometry (IMS) (separation)

- Infrared spectroscopy (IR)

○ Mass Spectrometry (MS) (low- and high-resolution)

- Triple quadrupole (low)

- Orbitrap (high)

- Theoretical

- Density Functional Theory (DFT) (quantum chemical calculations)

- Multivariate Statistical Analysis

- Analysis of Variance (ANOVA), multiple regression, principal components analysis, discriminant analysis), machine learning algorithm (e.g., logistic regression, neural networks), experimental design

- Software Proficiencies

○ JMP Pro ${ }^{\circledR}, R$-programming, Gaussian $(\odot)$ modeling software, AutoDock Vina, MassHunter ${ }^{\circledR}$ (qualitative and quantitative mass spectral analysis), ChemDraw, Xcalbur (mass spectral analysis)

\section{PUBLICATIONS}

Bell, S.*; Feeney, W. (2019) Single shot, single sample, single instrument detection of IGSR and OGSR using LC/MS/MS. Submitted to Forensic Science International, October 2018.

DOI: 10.1016/j.forsciint.2019.04.002

Feeney, W*; Vander Pyl, C; Bell, S.; Trejos, T. (2020) Trends in Composition, Collection, Persistence and Analysis of IGSR and OGSR: A Review. Submitted to Forensic Chemistry, April 2020. DOI: $10.1016 /$ j.forc.2020.100250 
Feeney, W*; Menking-Hoggatt, K; Vander Pyl, C; Ott, C; Arroyo, L; Bell, S; Trejos, T. (2021) Detection of Organic and Inorganic Gunshot Residues from Hands using Complexing Agents and LCMSMS. Submitted to Analytical Methods, June 2021. DOI: 10.1039/d1ay00778e

\section{IN PROGRESS}

Feeney, W*; Menking-Hoggatt, K; Curran, J; Arroyo, L; Bell, S; Trejos, T. (2021) Evaluation of Organic and Inorganic Gunshot Residues in Various Populations using LC-MS/MS. Submitted to Forensic Chemistry, October 2021.

Feeney, W*; Bell, S; Trejos, T. (2021) Investigations into host-guest interactions with metal ions using DFT for applications using mass spectrometry. In preparation to Journal of Physical Chemistry A, November 2021.

\section{PRESENTATIONS}

1. October 25-28, 2021. Korina Menking-Hoggatt; William Feeney*. Study of various types of Gunshot Residue and Background Populations using LIBS, Electrochemistry, SEMEDS, LC/MS/MS and probabilistic Interpretation. Brazilian Winter School Program. Webinar Presentation.

2. April 2, 2021. William Feeney*. Furthering the understanding and analysis of gunshot residue with host-guest chemistry and mass spectrometry techniques. Friday Science Short Division 643. NIST 2021. Webinar Presentation.

3. January 2021. William Feeney*. Detection of OGSR and IGSR from the Same Collection Stub using Complexing Agents and LC/MS/MS. Crossing Forensic Borders Seminar Series. Seminar Presentation.

4. December 2020. William Feeney*. Recent Advances and Incorporation of Combinational Analysis for OGSR and IGSR Identification. ASCLD Lightning Talk. Virtual Presentation/Invited Speaker.

5. February 2020. William Feeney*. Validation Detection Methodology from a Single Sample for OGSR and IGSR. American Academy of Forensic Science. Seminar Presentation.

6. April 2019. William Feeney*, Suzanne Bell PhD. Development of a Simultaneous Detection System for FDR. NIST 2019. Seminar Presentation.

7. October 2018. William Feeney, BS*; Suzanne Bell, PhD. Simultaneous Detection of Organic and Inorganic Firearm Discharge Residue using HPLC-QQQ and Host-Guest Chemistry. SciX 2018. Seminar Presentation.

8. August 2017. William Feeney, BS*; Suzanne Bell, PhD. Exhaustive Characterization of Firearm Discharge Residue using Mass Spectral Imaging and Time-of-Flight Secondary Ion Mass Spectrometry. 254th ACS National Meeting and Exposition. Seminar Presentation.

9. February 2017 William Feeney, BS*; Sydney Brooks, BS; Suzanne Bell, PhD. A Single Extraction, Consecutive Detection of Organic and Inorganic Firearms Discharge Residue Using Thermal Desorption Gas Chromatography/Mass Spectrometry (TD-GC/MS) and Inductively Coupled Plasma/Mass Spectrometry (ICP/MS). American Academy of Forensic Science. Poster Presentation.

10. February 2017. William Feeney*, BS; Lindsay Cheeseman; Suzanne Bell, PhD. Direct Thermal Desorption Coupled to Gas Chromatography/Mass Spectrometry (GC/MS) for 
the Characterization of Organic Firearm Discharge Residues. American Academy of Forensic Science. Poster Presentation.

11. February 2017. William Feeney, BS*; Sydney Brooks, BS; Brittany Yeager, BS; Suzanne Bell, $\mathrm{PhD}$. An Elemental Characterization of Firearms Discharge Residue Using Complexing Agents and Triple Quadrupole Mass Spectrometry (QQQ MS). American Academy of Forensic Science. Poster Presentation.

2020 NIJ - GRF Fellowship: Validation of a Single Instrument, Single Sample Protocol for the Detection of the Inorganic and Organic Constituents of Firearms Discharge Residue

2019 C. Eugene Bennett Fellowship

2018 C. Eugene Bennett Fellowship

\section{PROFESSIONAL AFFILIATIONS}

2020 AAFS Member

2019 Student-Run Seminar Series Organizer

2019 ForensicBites.org Author

2018 AAFS Member

2015 Phi Lambda Upsilon Member

\section{MENTORED UNDERGRADUATE RESEARCHERS}

Declan Revenew: Trained on LC-QQQ and performed daily maintenance. Analyzed comparisons between LC and GC

Lindsay Cheeseman: Trained on TD-GCMS software platforms and regular instrument maintenance. Helped develop extraction protocols and performed authentic firearm discharge residue sample collection. Collated data for efficient analysis.

Victoria Zeger: Trained on multiple instrumentation software platforms and collated data for efficient statistical analysis

Kylea Mathison: Trained on LC-MS and fluorescence reader software platforms. Assisted with extractions of authentic firing event samples and data collection. Developed methodology for analysis of metal-ligand complexes on microplate reader.

Caroline McCarthy: Trained on LC-QQQ software platforms and assisted with extractions of authentic firing event samples and data collection. Refined and created figures used in poster and seminar presentations and performed advanced data analysis.

Justin Chinsky: Performed daily tasks of sample preparation, data analysis, and statistical modeling for LC/MS.

Rachel Rutter: Trained on LC-QQQ and performed daily maintenance. Ran complex mixtures via infusion and developed methods for M-L complexes.

Amanda Meier: Trained on multiple instrumentation software platforms and collated data. 
Emily Halpern: Trained on LC-MS and fluorescence reader software platforms. Assisted with extractions of authentic firing event samples and data collection. Assisted in running organic compounds on plate reader and LC-MS.

WORKSHOPS AND CONTINUING EDUCATION

2020 Current Trends in Forensic Trace Analysis ( $3^{\text {rd }}$ Annual Virtual Symposium)

2020 FIS 703: Research Design for Forensic Science

2019 Host of Student-Run Seminar Series (Dr. Edward Sisco Research Chemist, National Institute of Standards and Technology

2019 Writing in the Sciences

2018 Basic Chemometrics

2018 Building a Vibrant Research Program

2018 Analytical Quality Control in a Microbrewery

2018 Agilent Sci-Dev Day 2018: Developing and Accelerating Your HPLC Expertise

2018 West Virginia University Workshop on Laser Ablation ICP-MS (LA-ICP-MS) and Laser Induced Breakdown Spectroscopy (LIBS)

2018 Webinar Agilent: OpenLab CDS Introductory Demonstration

2018 Webinar Agilent: Making the Leap: Small Molecules to Biomolecules

2018 Webinar ACS: Exceptional Presentations In Spite of PowerPoint

2018 Webinar ACS: Exceptional Presentations In Spite of PowerPoint: The Sequel

2018 Webinar Agilent: Dedicated and Verified Cannabinoid Potency Testing using the Agilent 1220 Infinity II HPLC System

2017 Agilent 6400 Series QQQ LC/MS Techniques and Operation - 4 Day (R1893A) Agilent Technologies 